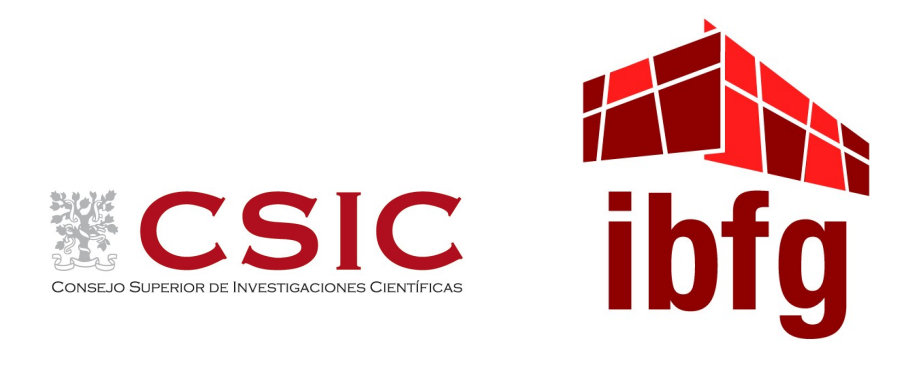

\title{
The role of $\mathrm{APC} / \mathrm{C}^{\mathrm{FZR}-1}$ in the \\ functionality of the gonad of
} Caenorhabditis elegans

\author{
Adrián Fragoso Luna
}

Instituto de Biología Funcional y Genómica CSIC-Universidad de

Salamanca

PhD Thesis

Salamanca, 2020 



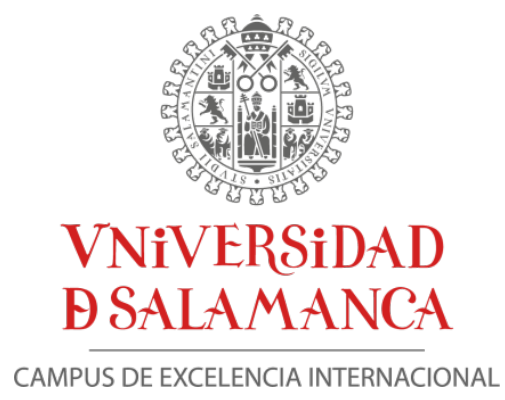

Universidad de Salamanca

Instituto de Biología Funcional y Genómica (IBFG-CSIC)

\section{The role of $A P C / C^{F Z R-1}$ in the functionality of the gonad of Caenorhabditis elegans}

PhD Thesis

Adrián Fragoso Luna

Salamanca, 2020 

Memoria presentada por Adrián Fragoso Luna para optar al título de Doctor por la Universidad de Salamanca.

Este trabajo ha sido realizado en el Instituto de Biología Funcional y Genómica (IBFG-CSIC) bajo la supervisión del Doctor José Pérez Martín. 

JOSE PEREZ MARTIN, DNI 28473540T, PROFESOR DE INVESTIGACION DEL CSIC EN EL INSTITUTO DE BIOLOGIA FUNCIONAL Y GENOMICA (CSIC-USAL)

\section{CERTIFICA:}

Que la memoria titulada "The role of APC/C FZR-1 in the functionality of the gonad of Caenorhabditis elegans", presentada por el Graduado ADRIAN FRAGOSO LUNA, ha sido realizada bajo su dirección en el Instituto de Biología Funcional y Genómica y reúne, a su juicio, originalidad y contenidos suficientes para que sea presentada ante el Tribunal correspondiente y optar al grado de Doctor por la Universidad de Salamanca.

Y para que así conste, a efectos legales, expide el presente certificado en Salamanca a 9 de Octubre de 2020.

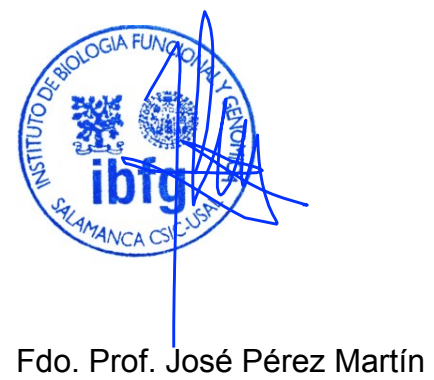





\section{Ackowledgments}

Durante la lectura de estas líneas haz que suene de fondo "How deep is your love" de los Bee Gees. Todo sentimiento necesita de una buena banda sonora. Gandhi dijo (o eso dicen) que lo que hagas en la vida será insignificante, pero que es muy importante que lo hagas, porque nadie más lo hará. Obviamente, esta tesis es algo insignificante para el mundo, pero para mí fue muy importante hacerla.

Este librillo es fruto del esfuerzo directo o indirecto de mucha gente, donde yo solo soy la punta del iceberg. Durante estos años en Salamanca he conocido a gente sensacional que me ha ayudado "mazo" y me ha enseñado de to' (que yo vengo de barrio chungo). Quisiera empezar agradeciendo al Dr/Prof José Pérez por brindarme la oportunidad de abrir un camino y enseñarme que el mundo que conocía antes de entrar en su labo solo eran sombras proyectadas en la pared de una cueva. Agradezco al Dr Jimbo por ser "Virgilio" en esta "Divina Comedia". A Sara, por enganchar el fenómeno. A David, por su cordialidad y buena disposición. A aquellos que también estuvieron: Paola, María y DJ Luigi, inventor de frases y número uno en el estilo macarra. Gracias de corazón. Una mención especial a Carmen Castro por enseñarme a ver lo invisible.

El lado divertido de la Ciencia lo representó el grupo MicroMundo: Bea, Ramón, Marga, Ricardo y Carlos. Gracias por esos momentos. Y en general, gracias a todos los componentes de la Caja Roja, sois gente formidable.

He de agradecer al legado musical de Elvis Presley que me brindara la necesaria paz para microinyectar y a los miles de millones de gusanos que dieron su vida sin siquiera saberlo. Doy las gracias de que ningún dios se enojase conmigo por no respetar los días sagrados, pero no tenía más remedio.

Durante estos años, el apoyo que me ha dado mi familia ha sido incondicional. Mis padres, mi hermana y el resto de mi familia. Gracias por hacerme profeta en mi tierra.

Cada pasito a lo largo de este abrupto camino solo fue posible a través de sangre, sudor y lágrimas. Rara vez la voluntad de los dioses coincidía con nuestros pensamientos. Debo confesar que si no abandoné el camino fue por Andrea, un ser angelical que me enseñó el verdadero significado de Eudaimonía.

\section{Gracias a TODOS.}

Y ahora, aquel gorrioncillo batió sus hermosas alas, siguiendo los vientos de cambio. 



\section{CONTENTS TABLE}

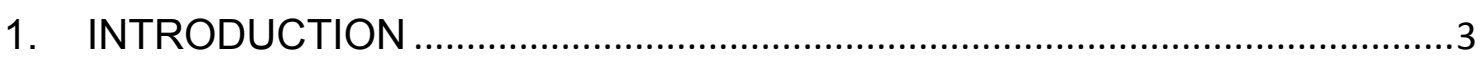

1.1. Development, an interplay between cell division and differentiation.......3

1.2. G1 phase offers a window opportunity to cell fate commitment...............4

1.3. $\mathrm{G} 1$ associated $\mathrm{E} 3$ ubiquitin ligase $A P C^{\mathrm{Cdh} 1}$ participates in differentiation

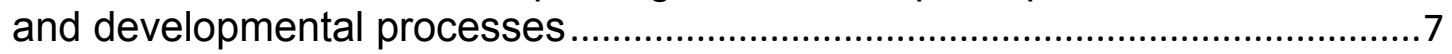

1.4. Caenorhabditis elegans overview.......................................................

1.5. C. elegans reproductive system ............................................................11

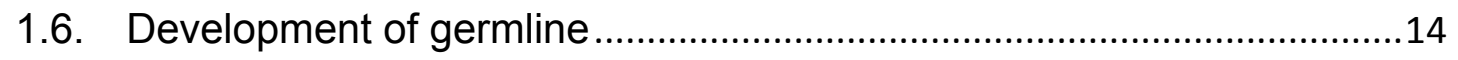

1.7. Chromatin regulators MES-4 and Polycomb Repressive Complex 2 (PRC2) are master regulators for maintaining germ cell identity......................15

1.8. Somatic gonad of $C$. elegans ................................................................. 18

1.9. Wnt signaling determines cell lineages within the somatic gonad.........21

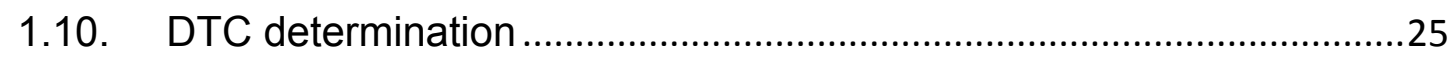

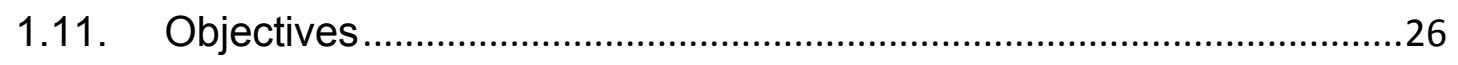

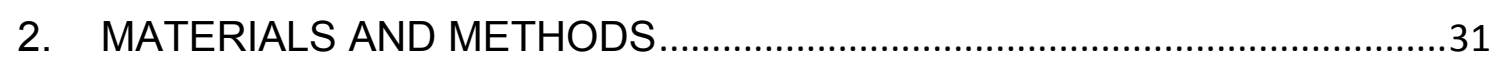

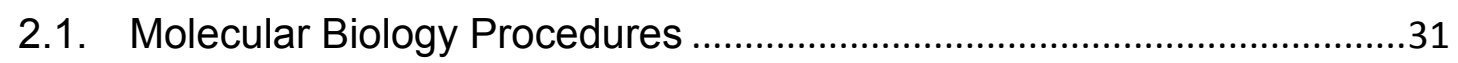

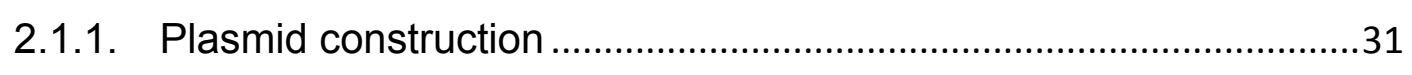

2.1.2. DNA extraction from C. elegans and PCR amplification...................31

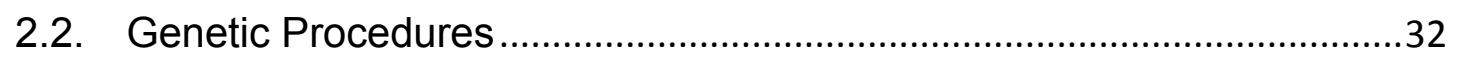

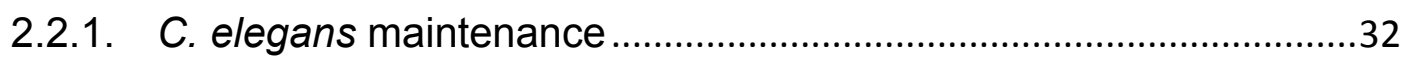

2.2.2. Generation of $C$. elegans strains by crossing.................................32

2.2.3. RNA interference (RNAi) experiments............................................32

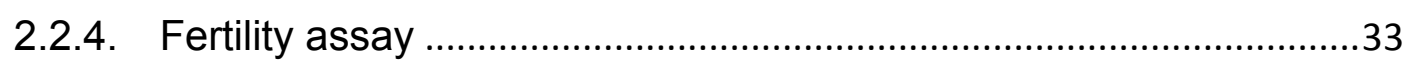

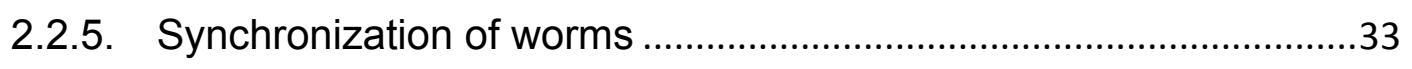

2.2.6. Microinjection of $C$. elegans germlines ...........................................33

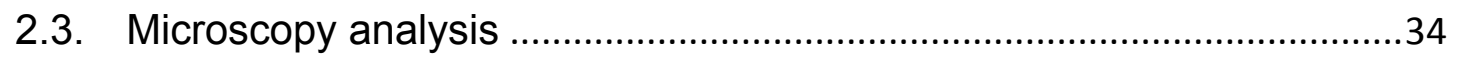

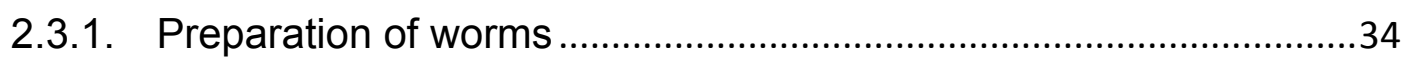

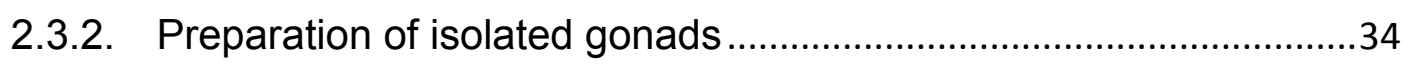

2.3.3. Analysis of in situ gonads by DAPI staining of worms .....................34

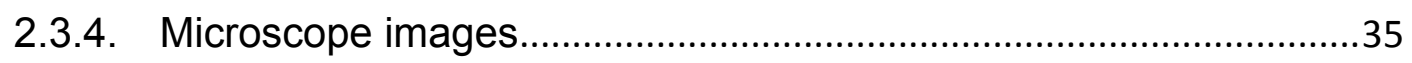

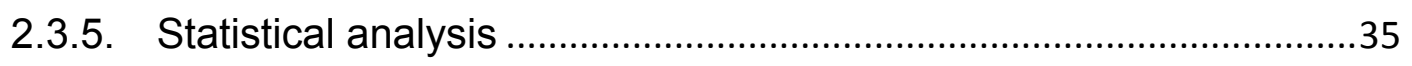

2.4. Description of the alleles constructed in this thesis ...............................35

2.4.1. mes-4(sal25[K10A, E11A, N12A])................................................35 
2.4.3. mes-3(sal12[mes-3::GFP + loxP 3xmyc::let-858utr + sqt(d) + hs::Cre + hygR^3+ loxP Flag::mes-3UTR]) and mes-3(sal13[mes-3::GFP + loxP Flag::mes-3UTR])

2.4.4. mes-3(sal13[mes-3(K37A, E38A, N39A)::GFP^3xFlag::mes-3UTR]) 38

2.4.5. mes-3(sal28[mes-3::tbb2UTR + unc-119 (+)]) I) ..............................38

2.4.6. mes-3(sal29[mes-3::GFP::tbb2UTR + unc-119 (+)]) I)....................39

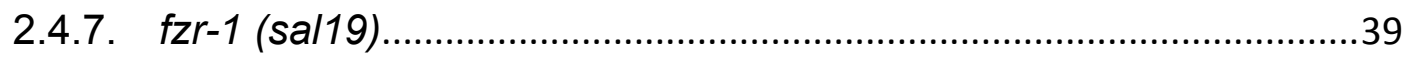

2.4.8. Construction and insertion of a fzr-1-expressing transgene............39

2.4.9. Construction and insertion of an inducible fzr-1-expressing transgene based on ribozyme-tetracycline system (fzr-1::rbz).

2.4.10. Construction and insertion of an inducible putative phospho-null fzr-1-expressing transgene based on ribozyme-tetracycline system (fzr18)::rbz) 41

2.4.11. Bipartite transgenic system used for marking somatic gonad cells 42

2.4.12. C. elegans strains and oligonucleotides used .43

3. RESULTS .51

3.1. MES-3 is a target of $\mathrm{APC} / \mathrm{C}^{\mathrm{FZR}-1}$ 51

3.2. APC/ $\mathrm{C}^{\mathrm{FZR}-1}$ and regulatory signals at the mes-3 $3^{\prime} \mathrm{UTR}$ collaborate to restrict the presence of MES-3 protein at early pachytene

3.3. The absence of degradation of MES-3 by APC/C $\mathrm{C}^{\mathrm{FZR}-1}$ dramatically affects fertility

3.4. The gonads of mes-3(AAA) worms yield defective eggs

3.5. The invasion of pachytene region could be responsible of fertility defects of mes-3(AAA)

3.6. LIN-35 and LIN-15B seems to interact distinctly with the nondegradable mes alleles.

3.7. $f z r-1$ (sal19) allele is a complete deletion of the $f z r-1$ coding sequence 64

3.8. $f z r-1$ (sal19) develop into sterile hermaphrodites .66

3.9. fzr-1(sal19) hermaphrodites failed to form gonadal arms. .68

3.10. Hermaphrodite fzr-1(sal19) worms were defective in distal tip cell formation.

3.11. Somatic gonad primordium was formed in fzr-1(sal19) .73

3.12. The absence of DTCs in fzr-1(sal19) cannot be explained by defects in the Wnt pathway.

3.13. The absence of DTCs is a consequence of cell lineage alterations in fzr-1(sal19) 
3.14. Expression of $f z r-1$ in Z1aa/Z4pp restores DTC production in fzr1(sal19)

3.15. The differences in cell fate between Z1aa and Z1ap are not attributable to different levels of FZR-1

3.16. A putative phospho-null version of $f z r-1(f z r-1(8 A))$ yields extra DTCs 86

3.17. Most likely, fzr-1(8A)-dependent extra DTC aroses from the differentiation of Z1ap to DTC.

3.18. The Wnt pathway could be controlling APC ${ }^{\text {FZR-1 }}$ activity by regulating CYE-1 asymmetry

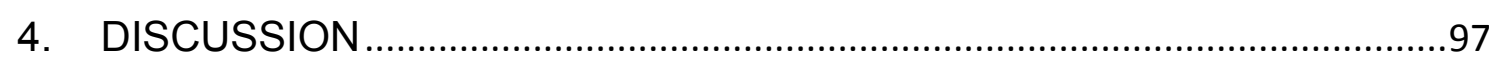

4.1. $\mathrm{APC} / \mathrm{C}^{\mathrm{FZR}-1}$ targets Polycomb subunit MES-3

4.2. Non-degradable MES-3(AAA) causes fertility defects that could come from the pachytene invasion of MES-3

4.3. Fertility defects associated with mes-3(AAA) are independent of LIN-35 (DRM/DREAM complex) but partially rescued by lin-15b mutants.

4.4. Loss-of-function of $f z r-1$ affects germline development only in hermaphrodites

4.5. $\mathrm{APC} / \mathrm{C}^{\mathrm{FZR}-1}$ determines DTC identity in hermaphrodites

4.6. Role of $A P C / C^{F Z R-1}$ in $\mathrm{mDTC}$ in $C$. elegans production and DTCs in related nematodes

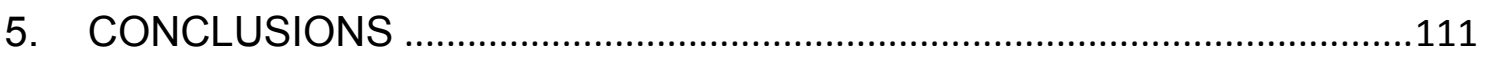

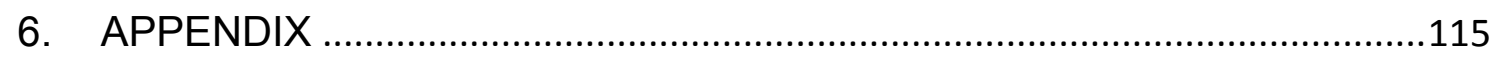

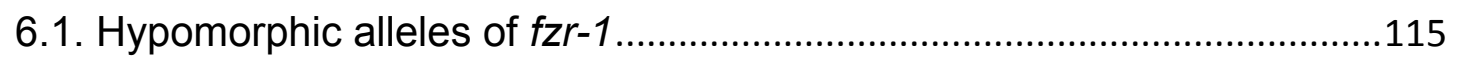

6.2. Bipartite transgenic system to track somatic gonad lineages .................116

6.3. Effects of chromatin regulators in the production of DTCs in fzr-1(sal19) 



\section{Abstract}

Proper coordination between the cell division and the differentiation is essential during the development of organisms. The cell-cycle machinery determines during the $\mathrm{G} 1$ phase, whether a cell differentiates or continues dividing to achieve this coordination. Many components of the cell cycle active in G1 can act directly on differentiation factors. Among these cell-cycle components highlights the $\mathrm{E} 3$ ubiquitin ligase $\mathrm{APC} / \mathrm{C}^{\mathrm{Cdh} 1}$ due to its emergent role in the degradation of several differentiation factors.

Caenorhabditis elegans constitutes and excellent system to study the possible roles of APC/C ${ }^{\text {Chh1/FZR-1 }}$ beyond cell cycle. Previous results from our laboratory demonstrated that $\mathrm{APC} / \mathrm{C}^{\mathrm{FZR}-1}$ sends to degradation the histone methyltransferase (HMT) MES-4. In this thesis, we have discovered that MES3 , a subunit of HMT Polycomb, is also targeted by APC/C $\mathrm{CZR}^{\mathrm{FZ}-1}$. We have observed that the low fertility levels associated with a version of MES-3 not recognizable by $A P C / C^{F Z R-1}$ are counteracted in combination with a version of MES-4 not degraded through $\mathrm{APC} / \mathrm{C}^{\mathrm{FZR}-1}$. The post-translational and simultaneous regulation of MES- 4 and MES-3 through APC/ $\mathrm{C}^{\mathrm{FZR}-1}$ constitutes a critical mechanism to ensure germline functionality.

On the other hand, we have explored the role of APC/C ${ }^{\text {FZR-1 }}$ during $C$. elegans development. We have obtained the first null-allele of fzr-1 described so far. APC/C ${ }^{\mathrm{FZR}-1}$ participates in the development of the somatic gonad, an organ that supports germline development. Specifically, APC/C ${ }^{F Z R-1}$ is necessary for the production of the Distal tip Cell (DTC), a stem-cell niche that maintains a pool of germ cells and leads the outgrowth of the gonad, Absence of FZR-1 makes that cells committed to being DTCs, acquire the SS precursor fate of sister cells. However, an allele of $f z r-1$ supposed to be constitutively active yields extra DTCs. Both results suggest that $A P C / C^{\text {FZR-1 }}$ is part of a balance during the fate choice decision 'DTC-SS' in the somatic gonad. 



\section{Abbreviation list}

A-P: Anterior-to-Posterior

AC: Anchor Cell

APC/C: Anaphase Promoting

Complex/Cyclosome

bHLH: basic Helix-Loop-helix

CDK 2/4/6: cyclin-dependent kinase

$2 / 4 / 6$

C. elegans: Caenorhabditis elegans

CKI: cyclin-dependent kinase inhibitor

CRISPR: Clustered regularly interspaced short palindromic repeats

CYE-1: Cyclin E

D-P: Distal-to-Posterior

DNA: Deoxyribonucleic Acid

DSB: Double Strand Break

DTC: Distal Tip cell

E.coli: Escherichia coli

EZH2: Enhancer of Zeste Homologous 2

Fig.: figure

FZR-1: Fizzy related

FZY-1: Fizzy

GFP: Green Fluorescent Protein

GSC: Germ Stem Cells

HR: Homologous Recombination

HTM: Histone Methyltransferase
HygR: Hygromycin B resistance

$\mathbf{K b}$ : kilobase

LC: Linker Cell

MAPK: Mitogen-Activated Protein

Kinase

MosSCI: Mos1-mediated single copy insertion

MES-4/2/3/6: Maternal Effect Sterile $4 / 2 / 3 / 6$

mRNA: messenger RNA

NHEJ: Non-Homologous End Joining

NSD: nuclear receptor-binding SET domain

PcG: Polycomb Group

PCR2: Polycomb Repressive Complex 2

PGC: Precursor Germ Cell

pRb: Retinoblastoma protein

PRC2: Polycomb repressive complex 2

Rb: Retinoblastoma

RNA: Ribonucleic Acid

RNAi: RNA of interference

SCF: Skp1, Cullin, F-box protein

SGP: Somatic Gonad Prmordium

sgRNA: single guide RNA

TZ: Transition Zone

UTR: Untranslated Region 




\section{INTRODUCTION}

\subsection{Development, an interplay between cell division and differentiation}

Development from a single cell into a complex multicellular organism constitutes a paradigm of biological organization. From a cell to give rise a multicellular organism, waves of cell division, and subsequent differentiation are needed. Therefore, correct development is only possible if division and differentiation are strictly controlled and coordinated. Alterations in cell division and differentiation lead to a plethora of diseases, including cancer (reviewed in (Boward et al., 2016).

Cell division is the process in which a cell gives rise to two daughter cells and is tightly regulated by the cell-cycle machinery. Cell cycle ensures that cells had duplicated their components before division has taken place. DNA replication, regarded as the central duplication event, occurs during $S$ phase (from "synthesis"). Cell division itself happens along with mitosis. Between the mitosis and S phase, there are two more phases, G1 and G2 (from "gap"). During $\mathrm{G} 1$ phase, cell senses external signals and decides if it divides or not, while internal inputs are analyzed in $\mathrm{G} 2$, checking that all intracellular components have been duplicated before entering mitosis. All these steps are promoted by the activity of Cyclin-dependent kinases (Cdk), which are associated with different partners of cyclins to form the CDK/cyclin complex (Nurse et al., 1976). The phosphorylation of key targets makes cells to move from phase to phase, completing a cell cycle round. Moreover, cell cycle proceeds in one single direction because CDK-dependent phosphorylations trigger irreversible events, including protein degradation (Swaffer et al., 2016). In lower eukaryotes, like fungi, a single catalytic Cdk subunit associates with different cyclins to promote the distinct cell cycle phases. In multicellular organisms, different catalytic subunits play distinct roles in the different cell cycle phases, interacting with specific cyclins (reviewed in (Hochegger et al., 2008). 


\subsection{G1 phase offers a window opportunity to cell fate commitment}

During development, cell cycle is far from being a fixed program, and actually it varies from pluripotent to differentiated cells (Fig. 1). Early in development, pluripotent cells, such as embryonic stem cells, follow rapid rounds of division. They alternate between $\mathrm{M}$ and $\mathrm{S}$ phases separated by short G1 and G2 phases. As pluripotent cells committed differentiation along with development, their G1 and G2 phases lengthen (reviewed in (White \& Dalton, 2005). In this regard, length of the $G 1$ phase seems to be an important feature of differentiating cells. Several independent factors converge to establish G1 phase as an 'opportunity window' to differentiate (reviewed in (Dalton, 2015) (Fig. 1). The central observation driving this concept is that $\mathrm{G} 1$ cells respond to specification signals more rapidly than do cells at other cell cycle positions. G1 cells are more sensitive to external cues, like growth or differentiation factors. The reasons for this behavior are not entirely clear. Although speculative, it is feasible that transcriptional programs linked to cell differentiation can be rapidly reset following exit from M phase (Singh et al., 2013). The transition from $M$ phase to $\mathrm{G} 1$ is associated with dramatic changes in nuclear architecture. Major genomic events occur during nuclear envelope reconstitution after mitosis, making developmental genes prone to be transcribed in $\mathrm{G} 1$. These include chromosome rearrangements, changes in epigenetic modifications, induction of DNA loops that bring together enhancers and promoters, thus facilitating transcription. Furthermore, transcription factors bind to developmental genes exclusively in $\mathrm{G} 1$. In summary, in the presence of pro-differentiation signals, G1 phase would potentially establish a favorable epigenetic and nuclear architectural environment that allows developmental programs to be activated (Walter et al., 2003, Singh et al., 2015, Singh et al., 2013). 


\section{Activation of developmental genes}

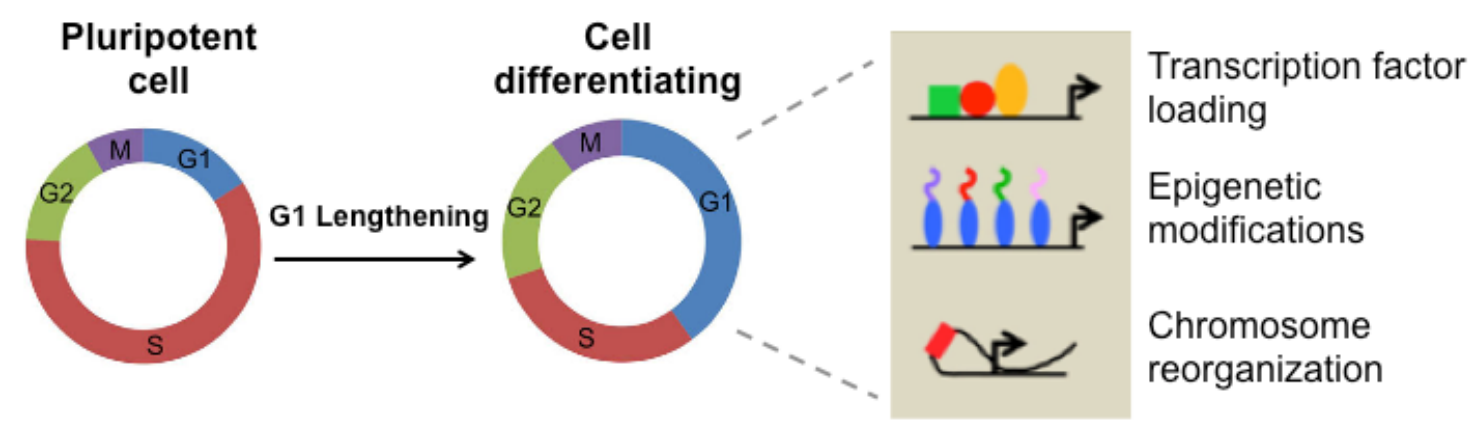

Figure 1. Cell cycle and differentiation are tightly coordinated. Cell cycle is composed of four phases: G1 ('Gap 1'), S ('Synthesis' of DNA), G2 ('Gap 2') and M ('Mitosis'). Early in development, pluripotent cells follow rounds of division in which $S$ phase and $M$ phase alternate after short Gap phases. Later on, cells committed to differentiate, alter their cell cycle due to lengthening of mainly, G1 phase, that is considered as a 'opportunity window' to differentiate. Many factors contribute to activate developmental genes specifically during G1: loading of transcription factors, epigenetic modifications and chromosome reorganization over developmental genes. Adapted from (Dalton, 2015).

In agreement with the role of G1 phase as an 'opportunity window' to fate commitment, differentiation programs force cells committed to differentiated to lock into G1 phase. These differentiation programs, responding to differentiation cues, are able to arrest cells by activating natural brakes that control the transition from $\mathrm{G} 1$ to $S$ phase. There are three major groups of inhibitors of G1/S transition operating at G1 (Fig. 2). The first one is the Retinoblastoma protein $(\mathrm{pRb})$. This protein binds to and inhibits the E2F-DP complex. E2F-DP, is a transcription factor required for the synthesis of factors involved in the $S$ phase, mainly DNA replication proteins as well as S-phase Cyclin E. By keeping E2F-DP inactivated, pRb prevented the progression through cell cycle (Geng et al., 1996). In addition, $\mathrm{pRb}$ is part of a chromatin modifier complex called DREAM, that resulted in a strong gene-silencing activity (reviewed in (Sadasivam \& DeCaprio, 2013)). $\mathrm{pRb}$ is negatively controlled by phosphorylation by the Cdk4/6-Cyclin D complex (Schade et al., 2019).

A second group of negative regulators of G1/S transition is composed of CDK inhibitors (CKI) (Fig. 2). These proteins directly bind to CDK complexes restricting its ability to phosphorylate their substrates. Two different families of CKI counteract CDK activity. The first group, the INK4 family, interacts with Cdk4/6-Cyclin D and therefore indirectly activated the pRb protein (Lukas et al., 1995, Guan et al., 1994). The second group, CIP/KIP family, inhibits the Cdk2- 
Cyclin E complex, which is the CDK that triggers S phase (Harper et al., 1993, Lee et al., 1995, Polyak et al., 1994). As it happens with pRb, these CKls are negatively regulated by phosphorylation by CDK complexes (Sheaff et al., 1997), resulting in the recognition by specific ubiquitin ligases complexes and its posterior degradation by the proteasome.

E3 ubiquitin ligases, which send proteins for proteasomal degradation, encompassed a third group of cell cycle inhibitors also participating in $\mathrm{G} 1 / \mathrm{S}$ transition, making the scheme more complex (reviewed in (Rizzardi \& Cook, 2012) (Fig. 2). There are two main groups of ubiquitin ligase complexes with distinct roles during G1/S progression. By one side, the SCF complex (composed of Skp1, Cullin, and F-box factor), which depending on its components, acts as a G1/S promoter or inhibitor. For instance, if complexed with Skp2, it was involved in the degradation of CIP/KIP members, while SCF complexed with Fbw7 targets Cyclin E (Koepp et al., 2001). A second E3 ubiquitin ligase, Anaphase-Promoting Complex or cyclosome (APC/C) with its co-activator Cdh1, negatively impinges on $\mathrm{G} 1 / \mathrm{S}$ transition counteracting the CIP/KIP degradation by targeting Skp2 (Bashir et al., 2004).

\section{G1/S transition}

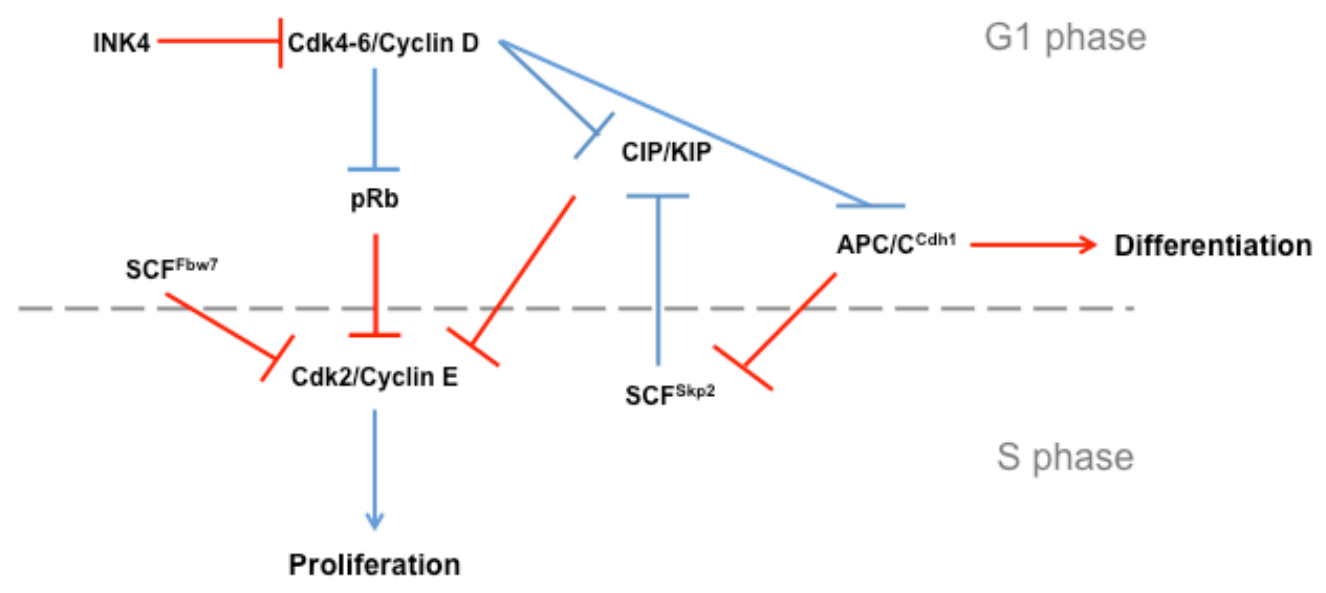

Figure 2. Regulation of G1/S transition. A complex network of cell cycle components determines if a cell starts a new round of division or remains quiescent in G1. Blue and red lines indicate reactions that promote and oppose G1/S transition, respectively. 


\section{3. $\mathrm{G} 1$ associated $\mathrm{E} 3$ ubiquitin ligase $A P C^{\mathrm{Cdh} 1}$ participates in differentiation and developmental processes}

$\mathrm{APC} / \mathrm{C}$ is a major E3 ubiquitin ligase involved in cell cycle regulation. Structurally, APC/C is a highly conserved complex of 15 to 17 subunits, depending on the organism. APC/C is divided into two subcomplexes held together by the scaffolding subunit, Apc1. The first of these subcomplexes attaches the substrate and the co-activator subunits, Cdc20 and Cdh1. These interactions involve some subunits carrying tetratricopeptide repeats, classical protein motives that participates in protein-protein interaction. The second subcomplex, which binds the E2 enzyme, consists of two subunits, Apc2, related to cullin proteins, and Apc11, the RING-finger protein (Thornton et al., 2006).

APC/C targets many proteins using its co-activators, Ccd20 and Cdh1, to achieve this task. These co-activators provide substrate-recognition specificity to APC/C. Also, the distinct regulation of these co-activators along the cell cycle is responsible of the timely activity of APC/C. Cdc20 is synthesized at the end of G2 phase, and it is degraded at the end of mitosis. In addition, the APC/C complex has to be previously phosphorylated at the beginning of mitosis by mitotic CDK in order to be able to bind Cdc20 (Kraft et al., 2003, Rudner et al., 2000, Rudner \& Murray, 2000). Altogether, these controls restrict the activity of $\mathrm{APC} / \mathrm{C}^{\mathrm{Cdc20}}$ to mitosis. In contrast, APC/C associated to Cdh1 is only active at the end of mitosis and early G1 phase, despite Cdh1 is present through all phases of cell cycle. However, along the major part of the cell cycle, Cdh1 is phosphorylated at multiple sites by CDK, impeding its interaction with APC/C. Only when the overall CDK activity dramatically decreases during late mitosis and early G1 phase, non-phosphorylated Cdh1 accumulated and binds to APC/C (Blanco et al., 2000, Kitamura et al., 1998). Furthermore, Cdh1 is inhibited by the specific repressor Emi1 in some systems (Miller et al., 2006).

The distinct regulation of APC/C co-activators reflects their roles during the cell cycle. APC/C, when complexed with the co-activator Cdc20, acts as an essential trigger of the chromatid separation and mitotic exit through degradation of Securin and mitotic cyclins, respectively (Cohen-Fix et al., 1996, Wäsch \& Cross, 2002, Yamano et al., 1998). In contrast, the role of APC/C ${ }^{\text {Cdh1 }}$ 
in cell cycle is more diffuse. Because its negative regulation by $C D K$, the $\mathrm{APC} / \mathrm{C}^{\mathrm{Cdh} 1}$ complex is not formed until end of mitosis, when APC/C ${ }^{\mathrm{Cdc} 20}$ triggers the destruction of all mitotic cyclins, resulting in disabled CDK, and leading to accumulation of dephosphorylated Cdh1 and, as a consequence, its binding to $\mathrm{APC} / \mathrm{C}$. Among the first substrates to be degraded by APC/C ${ }^{\mathrm{Cdh} 1}$ is $\mathrm{Cdc} 20$. In addition, $\mathrm{APC} / \mathrm{C}^{\mathrm{Cdh} 1}$ produces a robust mitotic exit through continuous degradation of cyclins and Skp2 (which as it was mentioned above, it was involved in the degradation of CKIs). All these events facilitate that cells exiting mitosis were retained in $\mathrm{G} 1$ phase. Inactivation of Cdh1 shortens G1 phase and, thus, results in premature entry into $S$ phase, which causes DNA damage (García-Higuera et al., 2008, Sigl et al., 2009). Also, the absence of Cdh1 resulted in lack of $\mathrm{G} 1$ arrest when cells were deprived of nutrients or mitogens or exposed to antimitotic signals (Castillo-Lluva et al., 2004).

The ability of $A P C / C^{C d h 1}$ to retain cells in $\mathrm{G} 1$ phase, also allows them to enter G0, a special phase where the cell has ceased mitotic division and keeps quiescent with non-replicated genome DNA. The G0 phase is a previous step to many differentiation programs, and because that, $\mathrm{APC} / \mathrm{C}^{\mathrm{Cdh} 1}$ has been considered as an important driver of differentiation in many cell types (Cappell et al., 2016). Several studies done in lower eukaryotes supported the role of $\mathrm{APC} / \mathrm{C}^{\mathrm{Cdh} 1}$ in developmental processes by its role elongating $\mathrm{G} 1$ phase length. Yeasts, in response to lack of nutrients, elongated its $\mathrm{G} 1$ phase enabling the activation of alternative developmental programs consisting in activation of mating, sporulation or virulence processes. In three distinct systems, Saccharomyces cerevisiae, Schizosaccharomyces pombe and Ustilago maydis, ablation of Cdh1 homologs (Cdh1/Hct1, Srw1/Ste9, and Cru1, respectively) leads to shortened $\mathrm{G} 1$ phase and as a consequence, defects in mating, sporulation and virulence (Castillo-Lluva et al., 2004, Kitamura et al., 1998, Schwab et al., 1997, Yamaguchi et al., 1997). In higher eukaryotes, the role of APC/C ${ }^{C h 1}$ participating in developmental decisions by promoting $\mathrm{G} 1 / \mathrm{G} 0$ phase, have also been supported by different studies, mainly during mammalian neurogenesis. These reports suggest a model in which $\mathrm{APC} / \mathrm{C}^{\mathrm{Cdh} 1}$ induces the differentiation of neural progenitors by extending G1 phase. However, it seems that the role of $A P C / C^{C d h 1}$ during differentiation in multicellular organisms is more complex than anticipated and that exceeds its ability to elongate $\mathrm{G} 1$ 
phase. There are many examples of $\mathrm{APC} / \mathrm{C}^{\mathrm{Cdh} 1}$ acting in developmental decisions aside of cell cycle.

APC/C $\mathrm{C}^{\mathrm{Cdh} 1}$ influences cellular differentiation by directly targeting various cell-specific transcription factors and their regulators for degradation. For instance, during myogenesis in mouse, APC/ $\mathrm{C}^{\mathrm{Cdh} 1}$ targets Myf5, a basic helixloop-helix $(\mathrm{bHLH})$ transcription factor that inhibits the myogenic fusion during differentiation into multinucleated muscle fibers (Li et al., 2007). In mammalian neurons, $\mathrm{APC} / \mathrm{C}^{\mathrm{Cdh} 1}$ targets the bLHL inhibitor $\mathrm{Id} 2$, to regulate neuronal morphology and activity (Lasorella et al., 2006). Also, it has been paradigmatic the discovery that $\mathrm{APC} / \mathrm{C}^{\mathrm{Cdh} 1}$ targeted SnoN, a transcriptional repressor of Smad proteins, which activated transcription in response to TGF-B family ligands (Stegmüller et al., 2008).

$\mathrm{APC} / \mathrm{C}^{\mathrm{Cdh} 1}$ also affected signaling pathways required for differentiation. The first evidence of such a crosstalk was the identification in Drosophila melanogaster that APC/C, when complexed with Fzr1 (D. melanogaster Cdh1 homolog), targets the kinase Nek2, which maintains the Wingless-signaling pathway active (Martins et al., 2017).

All these evidences indicate that the role of $A P C / C^{C d h 1}$ during developmental decisions is far from being clear and that further analysis is required, mainly in multicellular organisms. However, the essential role of Cdh1 for correct offspring observed in murine models (the ablation of Cdh1-homolog Fzr1 causes embryonic lethality due to abnormalities of the placenta) precludes this deeper analysis (García-Higuera et al., 2008). In this thesis, our aim was to analyze the roles of FZR-1 in the worm Caenorhabditis elegans.

\subsection{Caenorhabditis elegans overview}

C. elegans is a transparent and free-living nematode that were proposed as a model organism to study development (Brenner, 1974). It is easy to cultivate, feeding on bacteria layers on plates (Brenner, 1974). The life cycle of C. elegans is highly dependent on temperature. At $20^{\circ} \mathrm{C}$, its life cycle lasts 78 hours (h). However, at lower temperatures, such as $15^{\circ} \mathrm{C}$, it extends up to $125 \mathrm{~h}$ (Fig. 3). On the contrary, its life cycle shortens to $60 \mathrm{~h}$ when worms are incubated at $25^{\circ} \mathrm{C}$. Under environmental conditions favorable for reproduction, hatched larvae develop into adults after passing through four larval stages (L1, 
L2, L3, and L4). Adults could live for several weeks. (Byerly et al., 1976). Transitions to a new stage are preceded by a molting phase, during which worms produce a new external cuticle (Raizen et al., 2008). Besides, a particular resistant larval stage named "dauer" appears when food is scarce. Dauers survive without food for up to several months and could resume larval development after food supply (Golden \& Riddle, 1984).

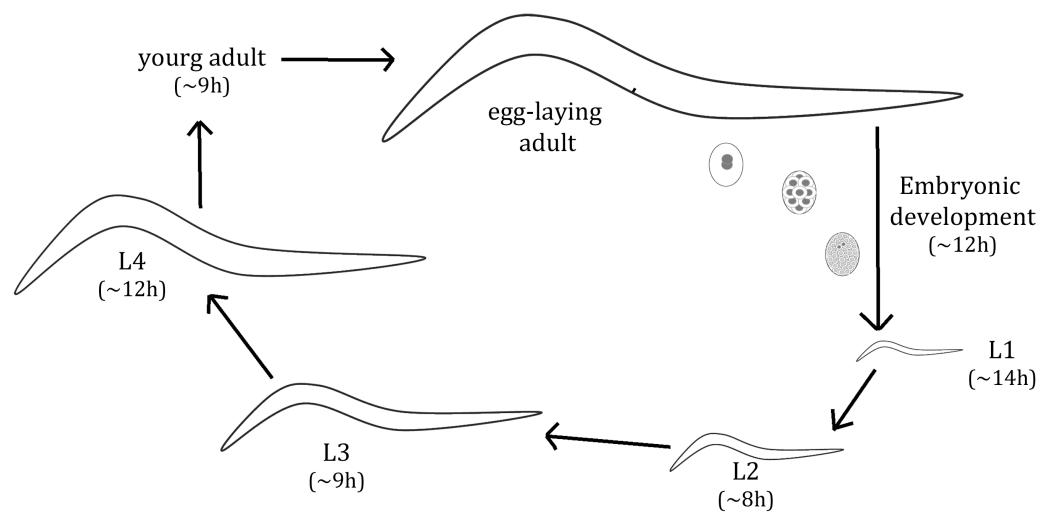

Figure 3. The life cycle of $C$. elegans.

C. elegans development is thoroughly characterized, as it is known the origin, position, and fate of every single cell. This detailed knowledge is explained because $C$. elegans has a fixed number of cells, meaning that cell lineage is almost invariant. In the gastrula stage, from 1-cell zygote to 550-cell stage, embryonic cells proliferate and arrange in a tubular pattern. Major rearrangements take place later on, throughout the stages of metamorphosis (bean stage) and elongation (comma and fold stages) (Sulston et al., 1983).

Anatomically, this nematode contains a digestive tract composed of a continuously pumping pharynx followed by an intestine. Its nervous system consists of a nerve cord. Most of its neurons are located in the head, behaving as chemosensors. Smooth-muscle tissues control the movement of organs such as the pharynx. Four bands of striated muscle and epithelial tissue outline its body and determine its sinusoidal displacement. A cuticle made of collagen covers the entire body and gives protection against external agents (Fig. 4). 


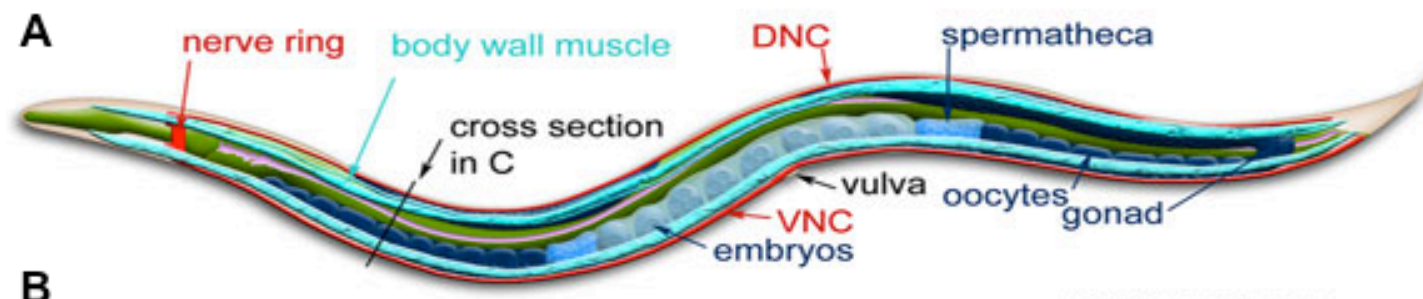

B

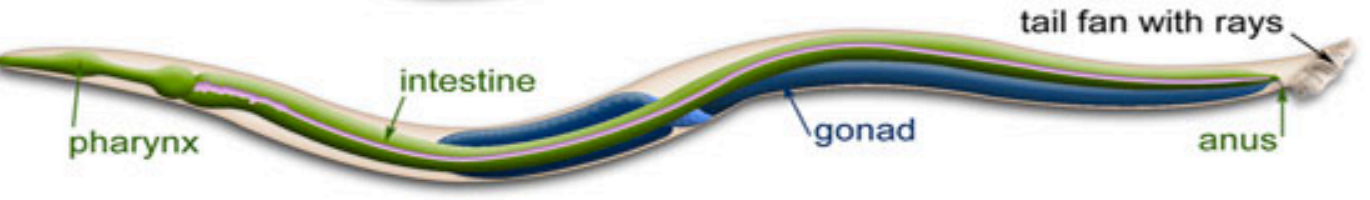

C

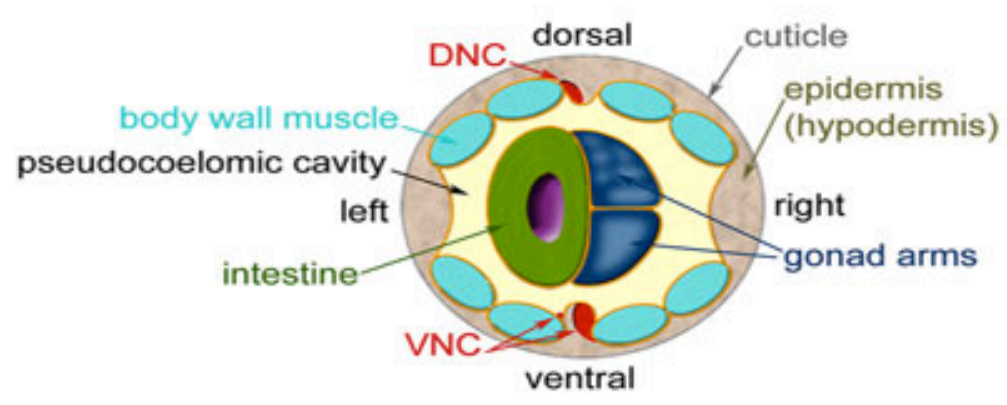

Figure 4. General anatomy of C. elegans. A. Longitudinal section of adult hermaphrodite B. Longitudinal section of adult male. C. Transversal section of hermaphrodite (https://www.wormatlas.org).

C. elegans is hermaphrodite, which makes easy the maintenance of clonal populations. In addition, hermaphrodites could yield a low rate of males (by random missing one of the two $X$ sexual chromosomes), roughly $0.1 \%$ among the total progeny (Hodgkin et al., 1979). Sexually dimorphic, C. elegans genders are visually distinguishable (Fig. 4). The main morphological feature of males is their copulatory apparatus used for mating. Adult hermaphrodites are composed of a total of 959 somatic nuclei, while males contain 1031 somatic nuclei (Sulston \& Horvitz, 1977). The presence of males makes possible sexual crosses, and therefore, genetic analysis.

\subsection{C. elegans reproductive system}

The reproductive system of $C$. elegans hermaphrodite is composed of two symmetrical U-shape gonads facing each other (Fig. 5). Initially, gonads produce a wave of sperm cells (roughly 150 per gonad) that were stored in a special organ called spermatheca. During L4, gonads cease sperm production and start to produce oocytes continuosly, which are fertilized when they cross the spermatheca to reach the uterus. Thus, upon self-fertilization, $C$. elegans produce eggs that are laid through the vulva (Fig. 5). A single hermaphrodite 
can produce 300 viable eggs, on average (Hirsh et al., 1976). If males fertilize hermaphrodites, the total progeny could be increased from 300 to roughly 1000 descendants (Hodgkin et al., 1979).

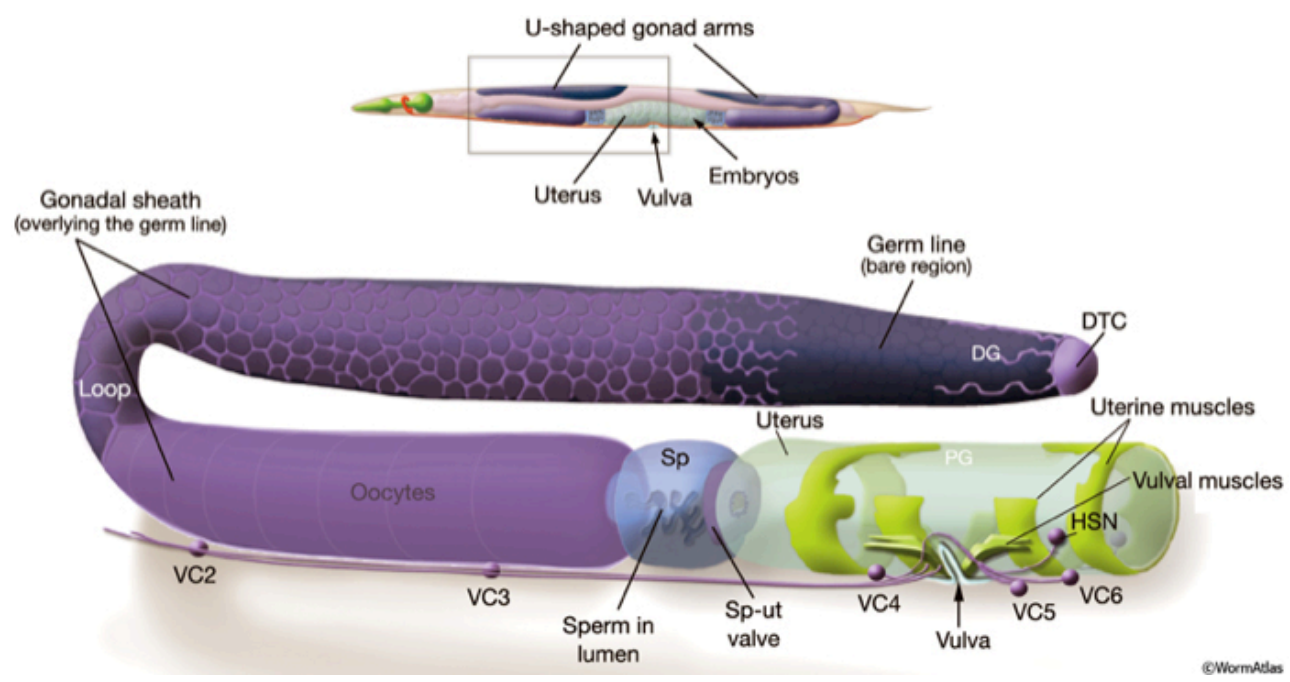

Figure 5. Reproductive system of C. elegans hermaphrodite. C. elegans contains two symmetrical Ushape gonads facing each other and connected through the uterus, where eggs/embryos are accumulated before being expelled. Each gonad is organized along a distal-to-proximal axis. At the distal end, a stemcell niche, the Distal Tip Cell, maintains a pool of mitotic germ cells (dark purple). Germ cells move from distal to proximal end. Once outside distal tip cell (DTC) influence, germ cells enter a meiotic program yielding oocytes. After passing through the spermatheca, oocytes are fecunded. Distal gonad (DG), spermatheca (Sp), spermatheca-uterine (Sp-ut) valve (https://www.wormatlas.org).

The adult germline exhibits distal-to-proximal (D-P) polarity. At one of its ends, the distal end, the germline possesses a pool of germ cells maintained in an undifferentiated and mitotic state. This pool of germ cells is actively maintained by signaling mediated by Notch pathway, provided by a stem-cell niche composed of the Distal Tip Cell (DTC), which caps this end of the germline. DTCs present a cup-like shape that extends processes towards germ cells (Byrd et al., 2014) (Fig. 6A). The plasma membrane of DTC expresses on its surface a Notch ligand, LAG-2 (Henderson et al., 1994). When DTC and germ cells are in tight contact, LAG-2 activates Notch receptor GLP-1 on the surface of germ cells (Kawasaki et al., 1998, Kimble \& Simpson, 1997). Once activated, GLP-1 self-cleaves releasing its cytoplasmatic fragment (Notch Intracellular Domain, NICD) that upon translocation to nucleus, forms a tertiary complex with LAG-1 DNA binding protein and LAG-3 transcription co-activator, 
activating the expression of distinct target genes producing RNA regulators (Chen et al., 2020, Petcherski \& Kimble, 2000). Among these, LST-1 (Nanoslike protein), SYGL-1 and the Pumilio RNA binding proteins, FBF-1 and FBF-2, are required to control the translation, mostly via repression, of a plethora of targets mRNAs. This widespread repression keeps germ cells in their proliferative as well as undifferentiated state (Fig. 6B) (Shin et al., 2017).

As germ cells divide, they advance through the germline towards the proximal end. Once germ cells are out of the influence of DTC, they turn off Notch signaling and as a consequence, it is induced the translation of mRNAs repressed by FBF-1/2 (Lamont et al., 2004). Among the proteins produced, NOS-3, GLD-1, GLD-2 and GLD-3 have major roles inducing the entrance in meiosis (Hansen et al., 2004). NOS-3 and GLD-1 seems to act together, repressing mitotic genes. GLD-1 is an RNA-binding protein that functions in translation repression, largely but not exclusively through 3' UTR regulation. NOS-3, which is an ortholog of the Nanos protein, cooperated with GLD-1 by a not well stablished manner (Hansen et al., 2004, Jan et al., 1999). GLD-2 and GLD-3 activate the expression of meiotic genes. GLD-2 is a cytoplasmic polyA polymerase that stabilizes $\mathrm{mRNA}$, while GLD-3 is an RNA-binding protein that brings GLD-2 to mRNA (Wang et al., 2002) (Fig. 6B).

Once meiosis program is activated, meiotic germ cells progress through distinct phases of the $1^{\text {st }}$ meiotic prophase. The shift from mitosis to meiosis occurs in the transition zone. This region features germ cells with crescentshape nuclei after staining of DNA. The transition zone contains germ cells in leptotene and zygotene phases. Pachytene stage coincides with the central region of the gonad, while diplotene happens in the loop that forms the germline. Finally, oocytes are stalked in diakinesis, until meiotic division re-stars after fertilization (Fig. 6C). 


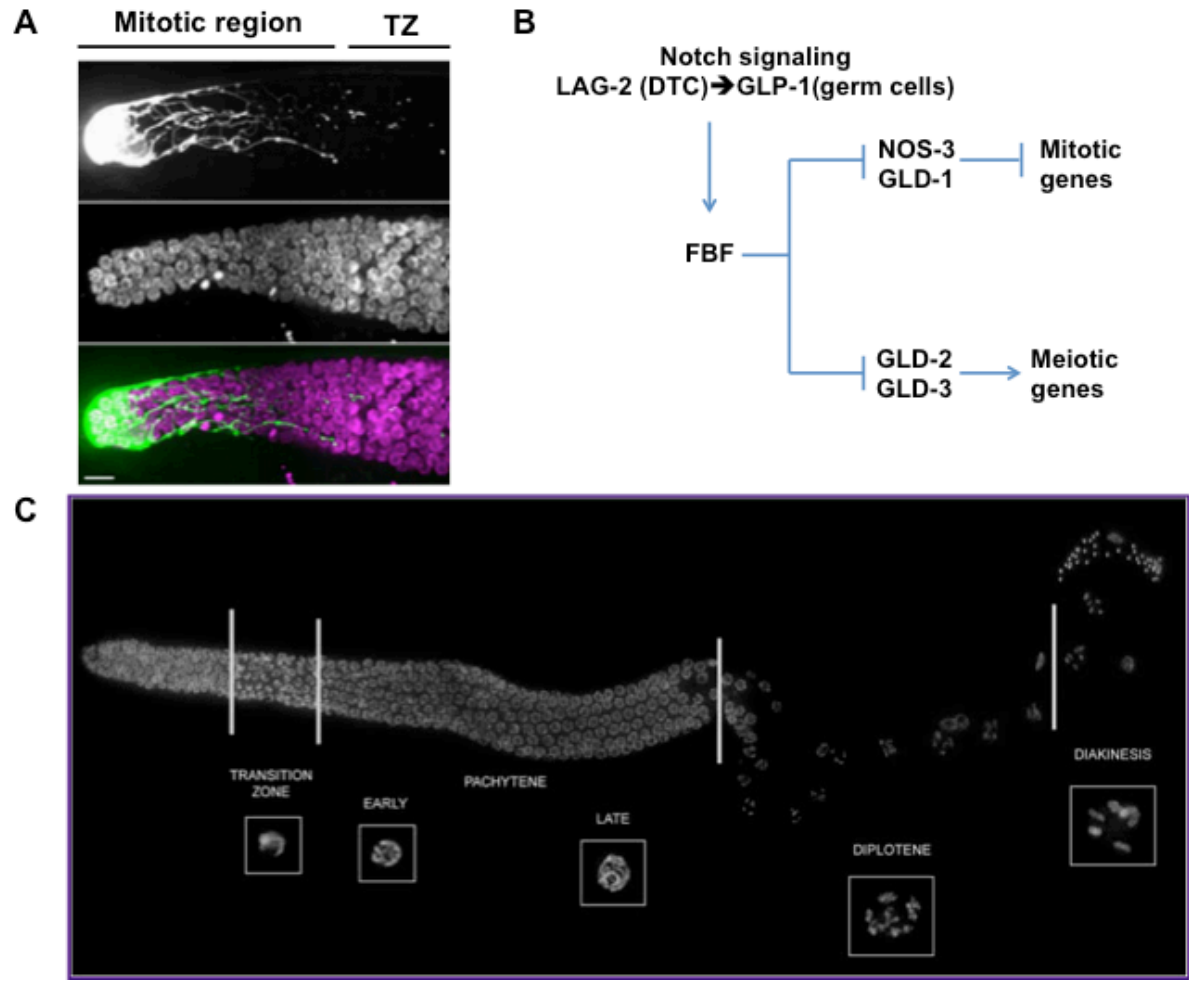

Figure 6. Distal Tip Cell (DTC) is a stem-cell niche that maintains germline. A. DTC is a stem-cell niche that extends processes and keeps a pool of mitotic germ cells (mitotic region). Outside the influence of DTC, germ cells enter meiosis in transition zone (TZ). Upper image shows a DTC expressing the reporter lag-2p::GFP. Middle image shows the germline with DAPI-stained nuclei. Lower image represents the merge (Linden et al., 2017). B. Notch ligand LAG-2, expressed in the surface of DTCs, activates Notch pathway in germ cells, maintaining their mitotic and germinal identity. LAG-2 ligand binds to GLP-1 receptor, located in the membrane of germ cells. GLP-1 self-cleavages and releases its intracellular domain, which acts as a transcription factor. Notch signaling induces the expression of Pumilo family member FBF, an RNA-binding protein that avoids translation of pro-meiotic mRNAs nos-3, gld-1, gld-2 and gld-3. After DTC, NOS-3 helps to activate the RNA binding protein GLD-1, which inhibits the translation of mitotic mRNAs, among others. On the other hand, the partner GLD-2/GLD-3 stabilizes meiotic mRNA due to its polyA activity. C. Extruded and DAPI-stained germline showing the different stages of meiotic prophase.

\subsection{Development of germline}

The germline of recently hatched L1 larvae is composed of two primordial germ cells (PGCs), named Z2 and Z3. Once L1 larvae start to feed, PGCs re-activate their cell cycle and start to divide. These cell divisions are sustained by Notch signaling provided by DTCs, which also lead the elongation of germlines. In L4, approximately 37 meiotic cells per arm at the most proximal end of the germline commit to sperm development. Subsequently, the germline 
switches from making sperm to making oocytes for the remainder of development and throughout adulthood (Fig. 7) (Kimble \& White, 1981).

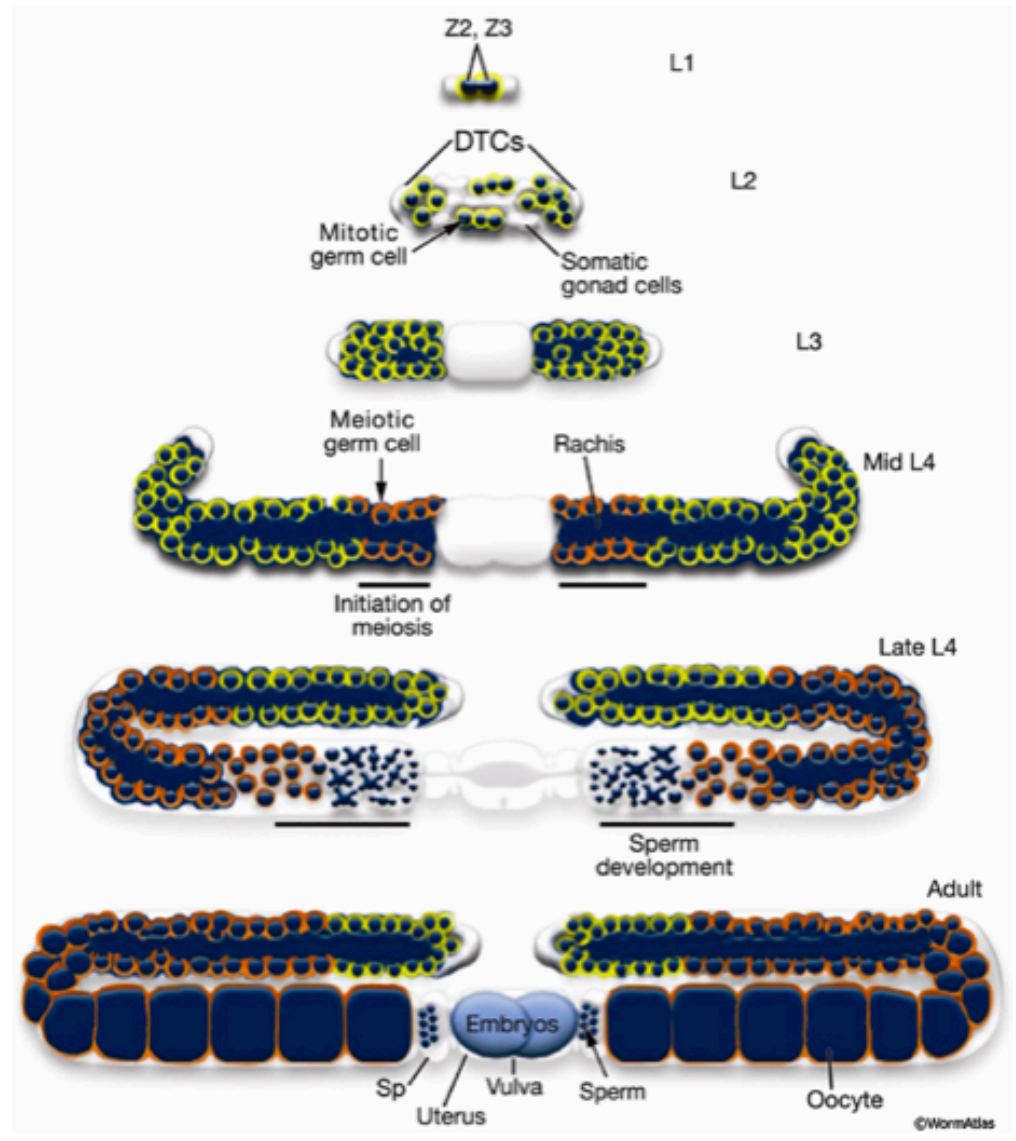

Figure 7. Germline development. Primordial germ cells (PGCs), Z2 and Z3, are the founder cells of the germline. Both cells start to divide mitotically through larvae stages. During L3, germline elongation commences guided by the Distal Tip Cell (DTC). Meiosis leading sperm production is activated at L4. Later on, meiosis switches from sperm to oocyte production, allowing self-fertilization.

\subsection{Chromatin regulators MES-4 and Polycomb Repressive Complex 2 (PRC2) are master regulators for maintaining germ cell identity}

Among the multiple levels of control that ensure the maintaining of germ cells, epigenetic regulation is a key factor. Germ cells possess a unique epigenetic landscape, which is the result of different chromatin modifications that allow the expression of germ identity genes, while somatic genes are kept silenced. Post-translational modifications of histone tails are an essential source of chromatin configuration. In C. elegans, this particular chromatin configuration 
is maintained by a series of proteins that were genetically defined as mutation having a maternal-effect sterile (MES): maternally provide MES product promotes development of a fertile germline, whereas absence of maternal MES product leads to death of nascent germ cells and sterile adults. MES proteins play an essential role in the already mentioned acquisition and upkeep of germinal chromatin. This group includes the members MES-2, MES-3, MES-4, and MES-6 proteins (Korf et al., 1998).

MES-4 contains a SET domain indicative of histone methyltransferase (HMT) activity and is homolog to the vertebrate NSD proteins. MES-4 generates H3K36me3 on genes expressed in the germline (Bender et al., 2006). MES-2, MES-3, and MES- 6 form the $C$. elegans version of the widely conserved Polycomb Repressive Complex 2 (PRC2) and generate repressive trimethylation at lysine 27 of histone 3 (H3K27me3) (Bender et al., 2004b). MES2 and MES-6 are the orthologs of $D$. melanogaster Enhancer of Zeste $[E(Z)]$ and Extra Sex Combs (ESC), respectively. The catalytic activity relies on the MES-2 subunit, which contains a SET domain (Ketel et al., 2005, Holdeman et al., 1998). Interestingly, the MES-3 subunit is exclusive of C. elegans, and ortholog proteins have not been found in any other organism (Xu et al., 2001).

MES-4 and PRC2 are necessary for germinal identity. MES-4-mediated tri-methylation at lysine 36 of histone 3 (H3K36me3) keeps the germinal genes of autosomes transcriptionally active. At the same time, PRC2-mediated H3K27me3 represses somatic gene expression. This particular combination of activation and repression maintain the identity of germ cells. In fact, mes mutants are prone to suffer transdifferentiation of germ cells into somatic cells upon induction of transcription factors (Patel et al., 2012).

MES-4 and PRC2 delimit their action to each other, as their methylation marks are mutually exclusive (Gaydos et al., 2012). This antagonism promotes appropriate gene expression in germ cells, allowing the correct disposition of each activating and repressive marks. A paradigmatic case to understand this behavior is the $\mathrm{X}$ chromosome, which in XX hermaphrodites and $\mathrm{XO}$ males is globally 'silenced' during most stages of germ cell development (Strome et al., 2014). MES-4 and H3K36me3 are enriched on the five autosomes and nearly absent from the $X$, whereas PRC2-generated methylation is modestly enriched on the X. Strikingly, in mes-4 mutants, X-linked H3K27 methylation decreases 
due to displacement of PRC2 complexes towards MES-4-controlled regions within autosomes (Fig. 8). Consequently, somatic genes within the sexual chromosome are de-repressed in such mes-4 mutants, producing sterility defects (Gaydos et al., 2012). In other words, it seems that in addition to maintain activating methylation in the germline genes, the role of MES-4 is also to repel PRC2-mediated methylation of germline-expresssed genes.

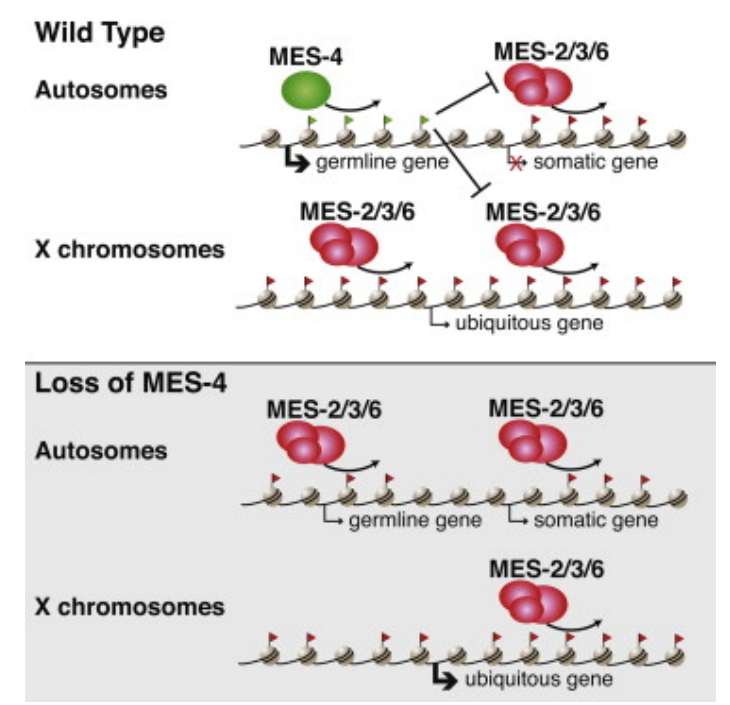

Figure 8. Histone methyl transferase (HMT) MES-4 delimits the activity of HMT Polycomb (MES2/3/6). In wild type germ cells, MES-4-dependent methylation is located mainly in autosomes, allowing the expression of germinal-associated genes. On the other hand, Polycomb-dependent methylation is concentrated within $X$ chromosome, leading to an overall silencing of somatic genes. Loss of MES-4 causes that Polycomb invades autosomes, extending its repressive mark along autosomes. At the same time, Polycomb-dependent methylation is diminished on $\mathrm{X}$ chromosome.

This antagonism explains the described similar distribution of MES-4 and MES-3 proteins along the germline: they are enriched in the mitotic distal region, dropping abruptly in the pachytene region to rise again in late pachytene and proximal region (Fig. 9). Interestingly, the molecular mechanism to maintain this similar distribution seems to be different. While in the case of MES-3, GLD1 seems to bind the 3' UTR repressing its translation in pachytene ( $\mathrm{Xu}$ et al., 2001) (Fig. 9B), for MES-4 the timely degradation by the APC/C ${ }^{F Z R-1}$ seems to be the responsible (Fig. 9A) (Rivera-Martín S, 2018). 
A
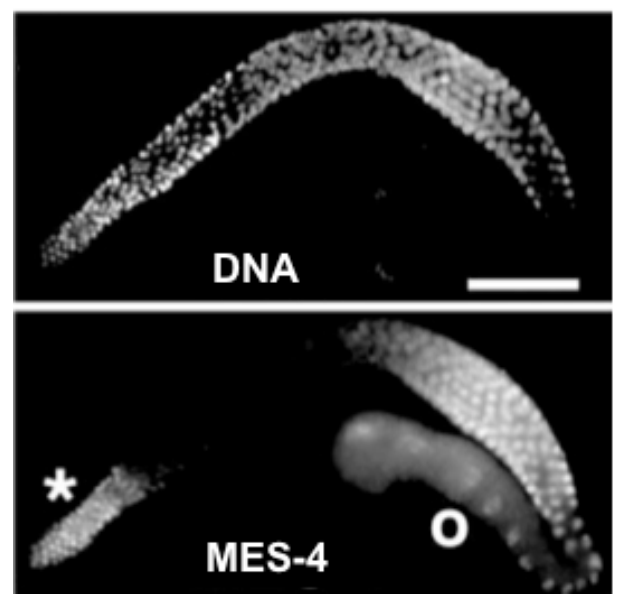

B
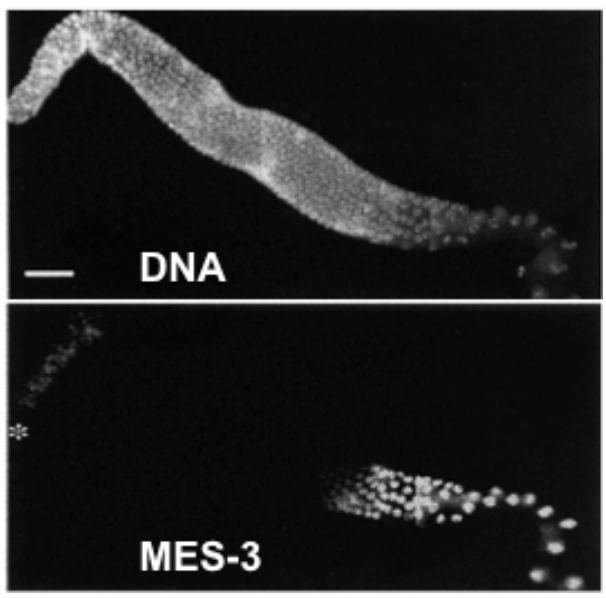

Figure 9. MES-4 and MES-3 proteins are expressed in proximal and distal regions of germline.

A. Germline stained with DAPI (upper image) and with antibodies anti MES-4 (Lower image). MES-4 protein levels drop in pachytene region (from (Fong et al., 2002)). B. Germline stained with DAPI (Upper image) and stained with antibodies anti MES-3 (lower image). MES-3 protein levels drop in pachytene region (from (Xu et al., 2001)).

\subsection{Somatic gonad of C. elegans}

Germline development and its maintenance depend on a support organ, the somatic gonad. In adult hermaphrodites, the somatic gonad is a complex tubular organ consisting of five tissues, each with specific functions and distinct anatomical features: the DTCs, gonadal sheath, spermatheca, spermathecauterine valve, and uterus (Fig. 10B) (Nigon \& Félix, 2017). Somatic gonad promotes the germline development through larval stages. This function mainly relies on two distinct cell types. In first place, the DTC, which is stem-cell niche that maintain germ cell identity (Byrd et al., 2014) and guides germline elongation. The second cell type that signals to germline are composed of sheath cells, which wrap the germline. One gonadal arm contains 10 sheath cells. It is known that sheath cells send signals to cells within germline, needed for proper oocyte production (McCarter et al., 1997). For instance, mutations in factors that alter the function of proximal sheath cells, like $x n p-1$, resulted in fertility defects (Bender et al., 2004a).

At hatching, the gonad comprises two primordial germ cells (PGCs), Z2 and Z3, flanked by the somatic gonad precursors (SGPs), named Z1 and Z4, and their surrounding basement membrane. These four cells remain mitotically 
quiescent until the mid-L1 when, upon feeding, PGCs and SGPs cells start to divide (Furuhashi et al., 2010). Initially, somatic gonadal cells and germ cells are mingled. Once that L2 stage is reached, all cell lineages that compose the somatic gonad are specified. In this stage, somatic gonad contains 12 cells. By L2, Z1/Z4 have generated 12 descendants: two DTCs, required for gonad elongation and germline patterning; nine blast cells that will, collectively, generate all other adult somatic gonad cells and one anchor cell (AC) a transient cell that induces vulval development (Kimble \& Hirsh, 1979) (Fig. 10A). Upon hatching, SGPs (either Z1 or Z4) start to divide asymmetrically along a D$\mathrm{P}$ axis. DTCs are the most distal cells, while the more proximal daughter resulted in one of the precursors of AC (Kimble \& Hirsh, 1979). These two more proximal cells (one per each gonad) have similar potential to become AC. Noisy variations in the expression of Notch receptor lin-12 between both equivalent cells make that the cell with higher lin-12 expression becomes ventral uterine (VU) cell, while the other becomes AC (Attner et al., 2019). Next, during L2/L3 transition, somatic gonadal cells and germ cells rearrange. DTCs move to the distal tips, the major part of somatic gonadal cells occupies the central region, and germ cells move toward both extremes. DTCs lead the outgrowth of germlines and sheath cells wrap the growing germline (Cecchetelli \& Cram, 2017). 
A

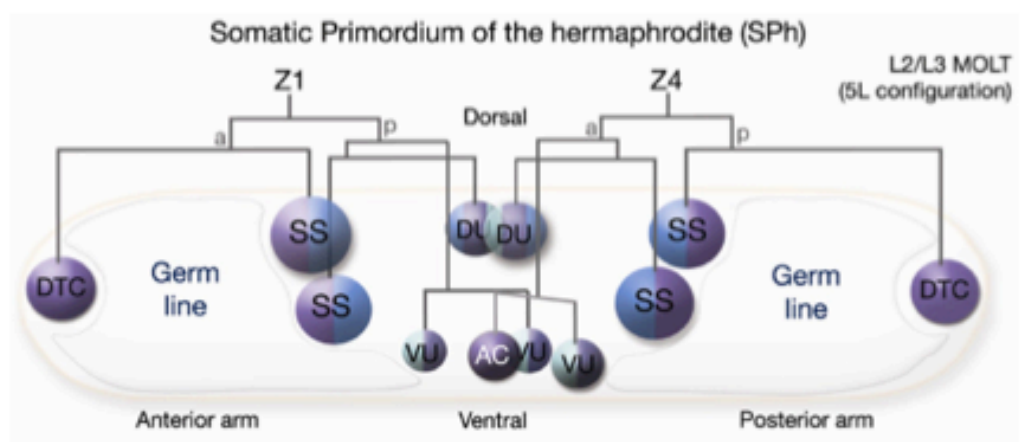

B

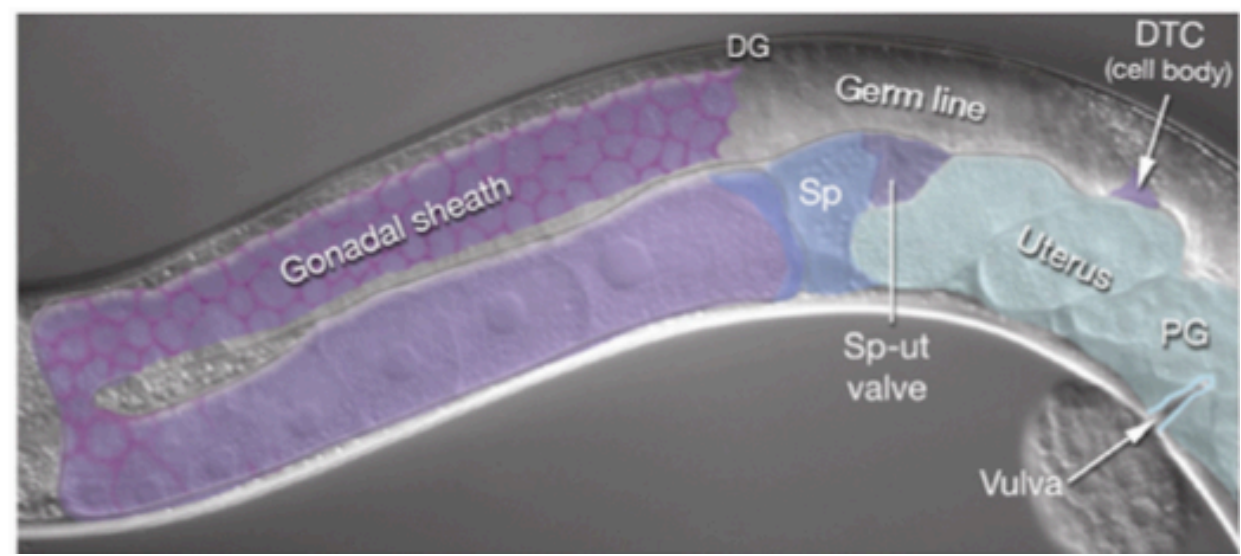

Figure 10. Somatic gonad of hermaphrodites. A. Somatic Gonadal Primordial Cells (SGPs), Z1 and Z4, are the founder cells of the somatic gonad. Both cells and their descendants divide asymmetrically through larvae stages yielding the different cell lineages. Somatic gonadal cell lineages are established during L2/L3 molt: Distal Tip Cell (DTC), Sheath-Spermatheca precursor cell (SS), Dorsal-Uterine cell (DU), Ventral-Uterine Cell (VU) and Anchor Cell (AC). B. Somatic gonad of adult hermaphrodite. Distal gonad $(\mathrm{DG})$, proximal gonad (PG) and Spermatheca (Sp).

C. elegans has evolved a different program for gonadal development in males. The gonad of $C$. elegans males consists of a single J-shaped arm (Kimble \& Hirsh, 1979) (Fig. 11). Male germline only produces sperm, which is released outside through the cloaca. Like hermaphrodites, male germlines contain a pool of undifferentiated and mitotically active germ cells in contact with the stem cell niche, composed of two male DTCs (mDTCs) located together in the distal gonad. Also, once they advance and get outside DTC influence, germ cells enter a meiotic program. At L1, the male gonad also comprises two PGCs, flanked by SGPs. 


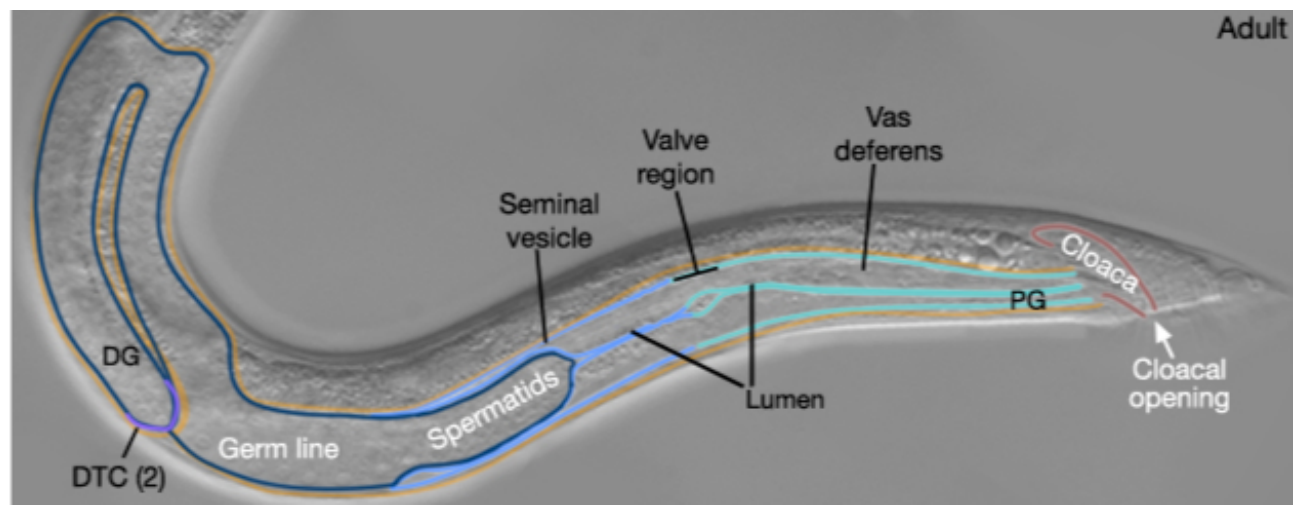

Figure 11. Male reproductive system. Males possess a single J-shaped gonad that produces sperm. Male somatic gonad consists of two DTCs located together in the distal region. Proximally, sperm cells are accumulated in the seminal vesicle. Sperm is released through cloaca after moving along vas deferens.

In contrast with hermaphrodite DTC, mDTCs do not lead the outgrowth of germline. mDTCs sustain the germ cell pool within the mitotic region while male gonadal outgrowth relies on the linker cell (LC), which is the leader cell (Kimble, 1981). By the L4 stage, LC reaches the cloaca at the posterior end, thus completing gonadal arm extension. After reaching the cloaca, LC enters cell death (Abraham et al., 2007). During gonadal outgrowth, the rest of male somatic gonadal cells advance with LC and produce the seminal vesicle and vas deferens, which allow the release of sperm through the cloaca (Fig. 11). Like AC, LC fate commitment stems from random decisions between two cells (Kimble \& Hirsh, 1979).

\subsection{Wnt signaling determines cell lineages within the somatic gonad}

Wnt signaling determines the fate of the different cells within somatic gonad throughout asymmetric divisions. Wnt pathway encompasses different subtypes. In drosophila and vertebrates, canonical Wnt pathway activates when extracellular Wnt ligand binds to Frizzled receptor and LRP6, both located in the plasma membrane. This ternary complex, in turn, recruits Dishevelled, that associated with destruction complex composed of four proteins: Axin, that is the scaffold subunit, kinases $\mathrm{CKI}$ and GSK3 $\beta$, and Adenomatous polyposis coli protein (APC). Upon membrane reclusion, this destruction complex is not able to target $\beta$-catenin, that accumulates and translocates into the nucleus, where it 
dimerizes with the transcription factor TCF, activating the expression of genes necessary for cell fate specification. In the absence of Wnt signaling, the destruction complex is continuously phosphorylating $\beta$-catenin, which is then ubiquitinated by $\beta$ Trcp and degraded by the proteasome. Monomeric TCF acts as a transcriptional repressor (reviewed in (Angers \& Moon, 2009)) (Fig. 12). In C. elegans, Wnt is used in many asymmetric divisions (reviewed in (Korswagen, 2002)). These divisions are organized according to an anterior-to-posterior (AP) axis. During development, the posterior daughter receives Wnt signaling while the anterior daughter does not activate the Wnt pathway.

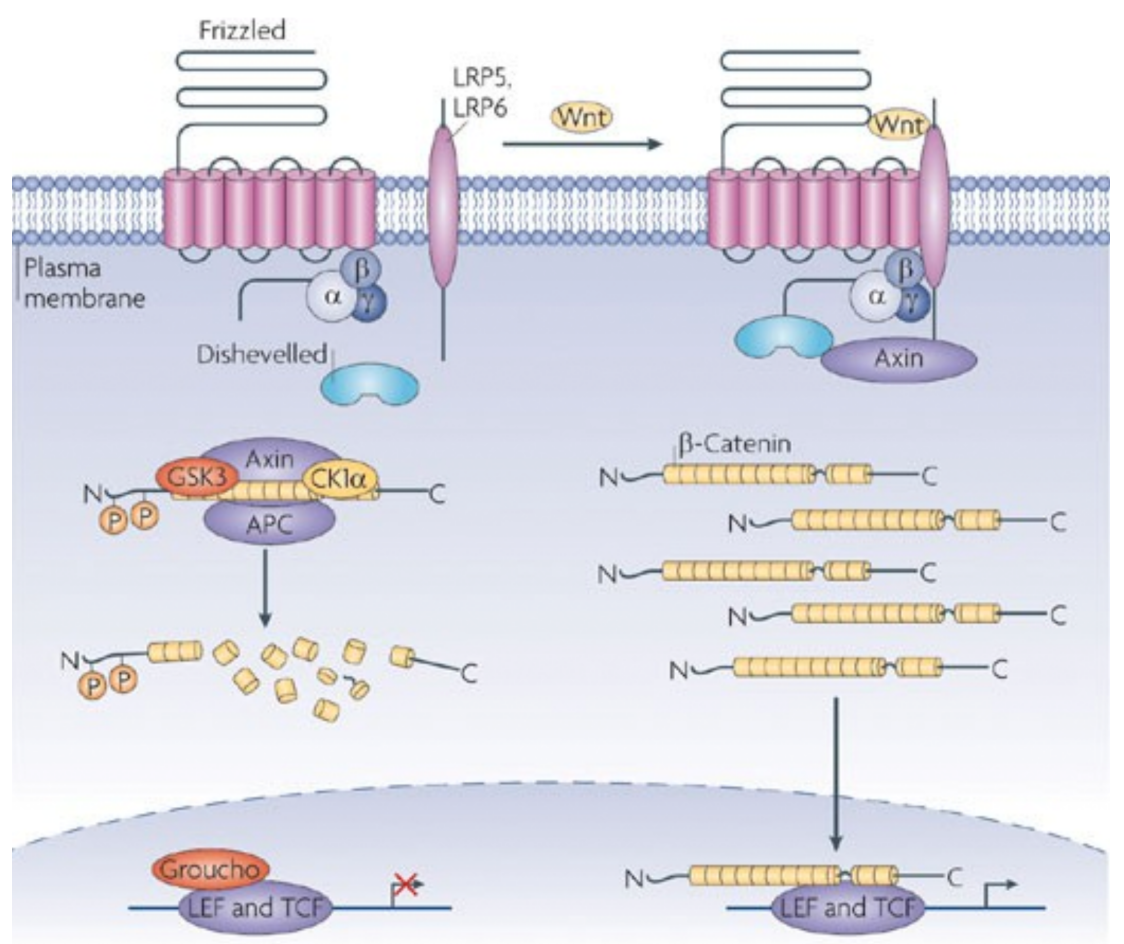

Figure 12. Canonical Wnt pathway. In absence of Wnt ligand, the destruction complex (Axin, GSK3,CKI and APC) phosphorylates and sends $\beta$-catenin (BAR-1 in C. elegans) for proteasomal degradation. Transcription factor LEF/TCP (POP-1) acts as a repressor of gene expression. When Wnt ligand binds to Frizzled receptor it triggers the recruitment of LRP5/6 and Dishvelled (DSH-2 and MIG-5), which in turn brings destruction complex to plasme membrane. $\beta$-catenin accumulates and translocates into the nucleus, where heterodimerizes with LEF/TCP, activating gene expression (from (Angers \& Moon, 2009)).

In contrast with the A-P body axis, the development of somatic gonad follows, instead, a D-P axis. After each cell division, Wnt pathway is active in the distal while posterior daughter keeps Wnt pathway off (Fig. 13A). In addition, somatic gonad development depends on a non-canonical Wnt pathway, the $W n t / \beta$-catenin asymmetry pathway. Molecular details of the $W n t / \beta$ - 
catenin asymmetry pathway are not fully understood (Phillips et al., 2007). The first enigma is that none Wnt ligand has been identified. Nonetheless, there are two described frizzled receptor, LIN-17 and MOM-5, which collaborates with Drosophila Disheveled homologs DSH-2 and MIG-5. The second enigma regards the destruction complex, which is not necessary to ensure correct Wnt signaling. Indeed, Wnt asymmetry pathway is not based on avoiding $\beta$-catenin SYS-1 degradation, but in its asymmetric delivery. Thus, Wnt-active distal daughter has a higher concentration of SYS-1 than its proximal sister (Phillips et al., 2007) (Fig. 13B and C). On the contrary, the nuclear levels of POP-1 are lower in distal daughter than in the proximal one (Fig. 13B and D). The reason is that Wnt asymmetry pathway bifurcates to regulate, on one side, the distribution of SYS-1, and on the other side POP-1. Wnt asymmetry pathway activates $\beta$-catenin WRM-1, which associates with the kinase LIT-1 and phosphorylates POP-1, translocating it from the nucleus to the cytoplasm (Siegfried et al., 2004, Siegfried \& Kimble, 2002). As a result, in the distal cell, the scarce nuclear POP-1 is bound to SYS-1, producing a heterodimer that activates the expression of downstream genes. The proximal cell has the opposite relationship, low levels of SYS-1, and high levels of POP-1. In this case, like in the canonical pathway, POP-1 acts as a transcriptional repressor (Fig 13B). SYS-1 is a special $\beta$-catenin, that showed no sequence similarity at amino acid level with canonical $\beta$-catenin, but whose tridimensional structures resembles canonical $\beta$-catenin, like WRM-1. Moreover, SYS-1 does not contain the motif recognized by the destruction complex, in agreement with the nonessential role of this destruction complex in $\mathrm{Wnt} / \beta$-catenin asymmetry signaling (Liu et al., 2008, Phillips et al., 2007). 


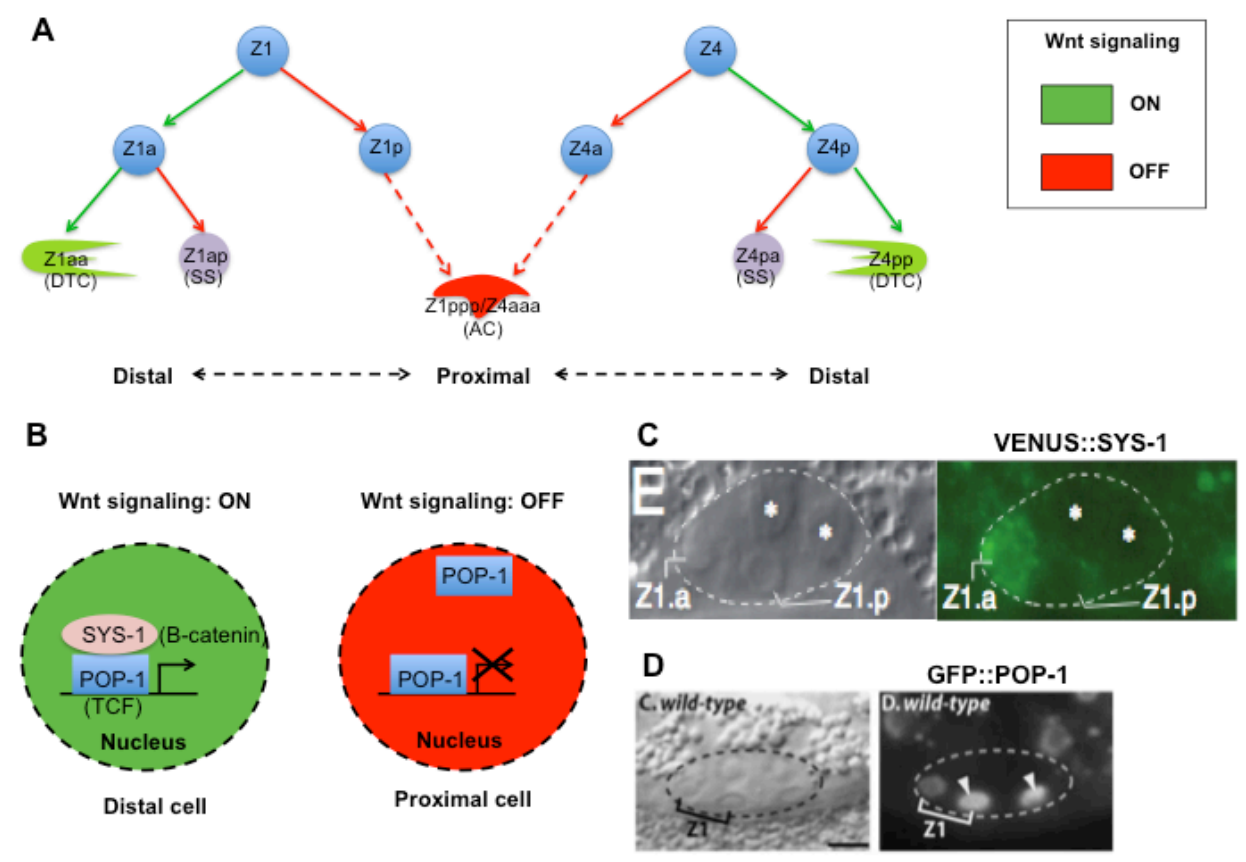

Figure 13. Non-canonical Wnt pathway participates in cell fate establishment within somatic gonad. A. Somatic gonadal cells divide asymmetrically along a distal-proximal axis. Distal and proximal daughter cells commit different fates. Non-canonical Wnt pathway is active and determines the fate of distal cells (green arrows). On the contrary, Wnt signaling is switched off in proximal cells (red arrows). B. After each division, non-canonical Wnt pathway controls the asymmetrical delivery of $\beta$-catenin SYS-1 and POP$1 /$ TCF between distal and proximal daughter cells. The nucleus of distal cell is enriched in SYS-1 while keeps low levels of POP-1. SYS-1 and POP-1 dimerizes activating the expression of genes controlling cell fate. On the other hand, the nucleus of proximal cell does not contain SYS-1 and retains higher levels of POP-1. In this case, POP-1 behaves as a transcriptional repressor. C. VENUS::SYS-1 reporter is present in distal cell Z1a but absent in proximal Z1p (from (Phillips et al., 2007)). D. GFP::POP-1 reporter is highly expressed in proximal cells Z1p and Z4a, than in distal cells Z1a and Z4p (from (Siegfried et al., 2004)).

Mutations in lin-17 or dsh-2 yield symmetric delivery of SYS-1 and POP1. Moreover, lit-1 and wrm-1 mutants also cause loss of POP-1 asymmetry (Phillips et al., 2007). Phenotypically, mutants in components of the Wnt pathway often lack one or both gonadal arms. These malformed gonads are the consequence of alterations in cell lineages within somatic gonad. Particularly affected are the most distal cell lineages, i.e., DTCs, which need a functional Wnt pathway. DTCs are frequently absent in the different Wnt mutants. On the other hand, in the most proximal cell lineage, the $A C$, Wnt pathway is never active, so this cell is always present in Wnt mutants. Indeed, extra AC is produced at the expense of DTCs. It means that in Wnt mutants, upon 
divisions, both daughters often acquire the same proximal fate. These symmetric divisions cause a lack of DTCs and gonadal arms, but the appearance of extra anchor cells, in what has been dubbed 'Sys' phenotype (Phillips et al., 2007, Chang et al., 2005, Siegfried et al., 2004, Siegfried \& Kimble, 2002).

Wht mutants also affect male gonadal development. However, hermaphrodites are more severely affected than males (Siegfried \& Kimble, 2002). For example, dsh-2 hermaphrodites lack at some frequency one or both gonadal arms due to missing DTCs. Moreover, they used to possess more than one AC. On the contrary, dsh-2 males elongate one J-shaped gonad, indicating that at least one $\mathrm{mDTC}$ is always produced. A more in-depth analysis showed the presence of extra LC, indicating D-P axis defects. dsh-2 also develop defective seminal vesicle and vas deferens (Chang et al., 2005).

\subsection{DTC determination}

Hermaphrodite DTC is the only somatic gonad lineage in which Wnt pathway is always active during the successive rounds of cell division. Wnt pathway in this case activates the hox gene ceh-22 (previously known as sys-3) that codes for a homeodomain transcription factor. A low level of expression of ceh-22 started before the first cell division in the SGP and it is strongly activated by Wnt pathway in the distal lineage (Z1a/Z4p) (Lam et al., 2006). The expression of ceh-22 remains in the DTC (Z1aa/Z4pp), while is repressed in the proximal sister (Z1ap/Z4pa) by a complex composed of the double bromodomain protein BET-1 and MYS-1 histone acetyltransferase (Shibata et al., 2014). Finally, ceh-22 expression is abrogated even in DTC between L2 and L3 transition, and secondary transcriptional factors maintain the DTC fate (Lam et al., 2006). Among the candidates are the basic-helix-loop-helix (bHLH) transcription factors LIN-32 and HLH-12, which are expressed in DTC and are required to maintain the DTC fate (Sallee et al., 2017).

DTC determination also seems to be under the control of cell cycle, since mutations in G1/S cell-cycle regulators affect the presence of DTC. For instance, cyd-1 mutants lack DTCs, and, consequently, also lack gonadal arms. Further analysis showed that CYD-1 acts at the level of SGPs regulating the 
Wnt pathway. In cyd-1 mutants, POP-1 asymmetry disappears, and therefore it mimics a defect in Wnt signaling (Tilmann \& Kimble, 2005). G1/S inhibitor CKI-1 also participates in DTC fate commitment. cki-1 RNAi resulted in the presence of extra DTCs. Furthermore, the effect of $\mathrm{CKI}-1$ depletion also alter other cell fates and for instance, cki-1 RNAi produces extra AC. Cell ablation experiments revealed that the ectopic DTCs could come from the division of the DTC itself, suggesting that the fates are not altered but the differentiated cells do not arrest their cell cycle. However, alternative explanations like that in the absence of $\mathrm{CKI}-1$ other somatic gonad cells commit DTC fate cannot be ruled out (Kostić et al., 2003).

An additional interesting connection between Wnt pathway and cell cycle regulation at the DTC, is the asymmetric distribution between Z1aa (DTC) and Z1ap (SS) of CYE-1, the S-phase cyclin. It seems that Wnt signaling keeps low CYE-1 levels to allow DTC fate acquisition. For instance, cye-1 mutants or cyeRNAi yields extra DTC because Z1ap and Z4pa do not differentiate into SS precursor cells but into DTC. Lack of DTCs upon ectopic expression of cye-1 further confirmed that CYE-1/CDK-2 opposites DTC fate. The molecular basis of this incompatibility is unknown (Fujita et al., 2007).

\subsection{Objectives}

It is necessary to achieve a deeper understanding of the complex interplay between cell cycle and differentiation processes. Results obtained in this field are relevant not only in the scientific sphere but also for potential medical applications. C. elegans is a multicellular organism easy to handle and well studied, offering an incredible system to study how cell cycle influences differentiation and vice versa. Previous results from our laboratory have demonstrated that the cell-cycle component APC/C ${ }^{\mathrm{FZR}-1}$ directs the proteasomal degradation of the histone methyltransferase MES-4. This non-cell cycle-related target described for APC/C $\mathrm{FZR-1}^{\mathrm{F}}$ paves the way to studying new roles of $A P C / C^{F Z R-1}$. To get more insight into the role played by $A P C / C^{F Z R-1}$ connecting cell cycle with differentiation, we decide to uncover new possible targets of APC/C ${ }^{\text {FZR-1 }}$ that participates in the maintenance and functionality of the gonad of $C$. elegans. For that, we have looked for additional targets of $A P C / C^{F Z R-1}$ in 
the germline of $C$. elegans. We also have characterized the consequences of the lack-of-function of FZR-1 in the development of the gonad. 

MATERIALS AND METHODS 



\section{MATERIALS AND METHODS}

\subsection{Molecular Biology Procedures}

\subsubsection{Plasmid construction}

Plasmids were first designed in silico with Serial cloner 2-6-1 software. Standard cloning protocols were applied (Maniatis et al.,1989). Competent Escherichia coli $\mathrm{DH} 5 \alpha$ was transformed with plasmids by the heat shock method (Hanahan, 1983). Plasmids were purified by alkaline lysis method (Birnboim \& Doly, 1979) and checked by digestion with restriction enzymes.

Plasmid pGEM-T easy (Promega) and pJET1.2 (Thermo Fisher) was used for subcloning and sequencing of genomic fragments generated by PCR. PCR fragments used for plasmid construction were first gel purified with QIAquick ${ }^{\circledR}$ Gel Extraction Kit.

\subsubsection{DNA extraction from $C$. elegans and PCR amplification}

DNA from C. elegans was extracted according to (Barstead et al., 1991). Worms were included in Lysis Buffer with $0.1 \mu \mathrm{g} / \mu \mathrm{L}$ of proteinase K. Samples were incubated at $-80^{\circ} \mathrm{C}$ for at least 5 minutes. Next, a reaction of $60^{\circ} \mathrm{C}$ during 1 hour and $95^{\circ} \mathrm{C}$ for 15 minutes was run in a thermocycler. DNA obtained was amplified with Taq DNA Polymerase for checking purposes or with high fidelity DNA polymerase Q5 (BioLabs), for plasmid construction and sequencing. Samples were run on TAE electrophoresis gels. Reaction mixes with the different polymerases and PCR programs are listed in table 1 and 2, respectively.

Table 1. PCR reaction mix

\begin{tabular}{|l|r|l|r|}
\hline \multicolumn{2}{|c|}{ Taq } & \multicolumn{2}{c|}{ Q5 } \\
\hline Reactive & $\mu \mathrm{L} /$ tube & Reactive & $\mu \mathrm{L} /$ tube \\
\hline Desionized water & 19,5 & Desionized water & 35 \\
Buffer 10x & 2,5 & Buffer Q5 5x & 10 \\
dNTPs $(10 \mathrm{mM})$ & 0,5 & dNTPs $(10 \mathrm{mM})$ & 1 \\
Oligo 1 $(100 \mu \mathrm{M})$ & 0,125 & Oligo 1 $(100 \mu \mathrm{M})$ & 0,5 \\
Oligo 2 $(100 \mu \mathrm{M})$ & 0,125 & Oligo 2 $(100 \mu \mathrm{M})$ & 0,5 \\
Taq & 0,25 & Q5 $(2 \mathrm{u} / \mu \mathrm{L})$ & 1 \\
DNA & 2 & DNA & 2 \\
\hline Final volume & 25 & Final volume & 50 \\
\hline
\end{tabular}


Table 2. PCR program

\begin{tabular}{|c|c|c|c|}
\hline & \multicolumn{3}{|c|}{ PCR program } \\
\hline & Polymerase & Taq & Q5 \\
\hline Step $1(x 1)$ & Denaturation & $94^{\circ} \mathrm{C} 5 \mathrm{~min}$ & $98^{\circ} \mathrm{C} 30 \mathrm{~s}$ \\
\hline & Denaturation & $94^{\circ} \mathrm{C} 30 \mathrm{~s}$ & $98^{\circ} \mathrm{C} 10 \mathrm{~s}$ \\
\hline Step $2(x 30)$ & ) Annealing & $60^{\circ} \mathrm{C} 30 \mathrm{~s}$ & $55^{\circ} \mathrm{C} 20 \mathrm{~s}$ \\
\hline & Elongation & $68^{\circ} \mathrm{C} 4 \mathrm{~min}$ & $72^{\circ} \mathrm{C} 4 \mathrm{~min}$ \\
\hline Step $3(x 1)$ & Elongation & $68^{\circ} \mathrm{C} 7 \mathrm{~min}$ & $72^{\circ} \mathrm{C} 2 \mathrm{~min}$ \\
\hline
\end{tabular}

\subsection{Genetic Procedures}

\subsubsection{C. elegans maintenance}

Strains were derived from Bristol N2 wild type and maintained using standard methods (Brenner, 1974). Basically, worms were maintained at $20^{\circ} \mathrm{C}$ on nematode growth media spotted with Escherichia coli OP50. Nematode growth media contained $3 \mathrm{~g} / \mathrm{L} \mathrm{NaCl}, 2.5 \mathrm{~g} / \mathrm{L}$ peptone, $20 \mathrm{~g} / \mathrm{L}$ agar, $25 \mathrm{ml} / \mathrm{L} 1 \mathrm{M}$ potassium phosphate buffer $\left(1 \mathrm{M} \mathrm{K}_{2} \mathrm{HPO}_{4}\right.$ mixed with $1 \mathrm{M} \mathrm{KH}_{2} \mathrm{PO}_{4}$ to reach a $\mathrm{pH}$ of 6.0$), 1 \mathrm{mM} \mathrm{CaCl}, 1 \mathrm{mM} \mathrm{MgSO} 4$, and $5 \mu \mathrm{g} / \mathrm{mL}$ cholesterol.

\subsubsection{Generation of $C$. elegans strains by crossing}

For crossing, eight to ten $C$. elegans males from one strain were mated with three to four hermaphrodites of another strain. $C$. elegans males were obtained upon 6 hours of heat-shock at $32^{\circ} \mathrm{C}$ of L4 worms. Offspring was isolated in L4 or previous stages avoiding them to be fertilized by males. To distinguish the hermaphrodite self-progeny from the progeny resulting from mating, PCRs of the isolated F1 worms or tracing of fluorescence markers were used.

\subsubsection{RNA interference (RNAi) experiments}

RNAi feeding method was used for gene silencing (Timmons \& Fire, 1998). RNAi-producing plasmids were obtained from from Ahringher's library (Kamath \& Ahringer, 2003) or constructed ad hoc using pL4440. To prepare RNAi feeding dishes, Escherichia coli (E. coli) HT115 strain transformed with RNAi-producing plasmid was grown in Luria-Broth (LB) medium with ampicilin $(200 \mu \mathrm{g} / \mathrm{mL})$ at $37^{\circ} \mathrm{C}$ overnight. $100 \mu \mathrm{L}$ of a $10 \mathrm{x}$ concentrated culture were seeded onto dishes with NGM medium, IPTG $(50 \mu \mathrm{g} / \mathrm{mL})$ and ampicillin (200 
$\mu \mathrm{g} / \mathrm{mL}$ ). Dishes were incubated at room temperature overnight to allow double strand RNA (dsRNA) production.

$\mathrm{L} 4$ worms were placed at $20^{\circ} \mathrm{C}$ or $25^{\circ} \mathrm{C}$ on RNAi plates, spread with the appropriate RNAi bacterial strain (Kamath et al., 2001). The next day, adults were transferred to fresh plates and left to lay eggs for $2.5 \mathrm{~h}$ before being removed. The following days, the development of the progeny observed. Each experiment was repeated at least three times. To test the effect of RNAi at the L1 stage, we grew worms on control RNAi plates as above but transferred their progeny at the L1 stage to plates spread with the appropriate RNAi bacterial strain.

\subsubsection{Fertility assay}

For viability and brood counts, single L4 worms were picked to plates and transferred to new plates every $48 \mathrm{~h}$ until egg-laying stopped. After removal of the parent, the number of live progeny was counted for two days.

\subsubsection{Synchronization of worms}

To obtain synchronized populations, adult hermaphrodites were bleached, allowed to hatch overnight in S-medium, and synchronized L1 worms were provided food and grown at the desired temperature. After $46 \mathrm{hr}$ at $20^{\circ} \mathrm{C}$, L4-adult molting worms were picked to a separate plate and were used for dissection after 8 hr (Porta-de-la-Riva et al., 2012).

\subsubsection{Microinjection of C. elegans germlines}

For Mos-mediated integration of transgenes we use Mos-universal system followed the procedures described in the Jorgensen webpage (https://wormbuilder.org/old/). For CRISPR we used in vitro assembly of Cas9 RNP complexes following procedures described in (Vicencio et al., 2019)).

We have injected CRISPR or Mos mixes into germlines of 1 day-old adult worms grown at $20^{\circ} \mathrm{C}$. We used a Nikon ECPLISE Ti microscope in combination with a FemtoJet ${ }^{\circledR}$ Microinjector and a PatchMan ${ }^{\circledR}$ NP2 micromanipulator, both from Eppendorf, and Femtotips ${ }^{\circledR}$ II capillaries. Every injection into germline was done with a pressure of $2500 \mathrm{hPa}$ during 0.2 
seconds. Worms were put on a $2 \%$ agarose pad (where they remained immobilized) and covered with Halocarbon oil.

\subsection{Microscopy analysis}

\subsubsection{Preparation of worms}

Worms were pre-incubated for 5 minutes in M9 solution containing 0.25 $\mathrm{mM}$ levamisol and $0.1 \%$ Tween-20, in order to anesthetized them. Worms were put on a $2 \%$ agarose pad. Then, mounting oil vectashield was added. The sample was mantled with cover slips and sealed with polish nail.

\subsubsection{Preparation of isolated gonads}

Worms were pre-incubated for 5 minutes in M9 solution containing 0.25 $\mathrm{mM}$ levamisol and $0.1 \%$ Tween-20, in order to anesthetized them. Gonads were isolated by extrusion after chopping off heads of worms with a scalpel. The nuclei of the gonads were stained with DAPI (4',6-diamidino-2-phenylindole) after permeating them with methanol during 5 minutes at $-20^{\circ} \mathrm{C}$ and washing three times with a phosphate saline buffer (PBS 0.1\% Tween 20) containing $0.1 \%$ tween-20. DAPI was added at a final concentration of $50 \mu \mathrm{g} / \mathrm{mL}$. Gonads were incubated for 30 minutes in darkness at room temperature. Finally, stained gonads were put on a $2 \%$ agarose pad. Then, mounting oil vectashield was added. The sample was mantled with cover slips and sealed with polish nail.

\subsubsection{Analysis of in situ gonads by DAPI staining of worms}

Worms were pre-incubated for 5 minutes in M9 solution containing 0.25 $\mathrm{mM}$ levamisol and $0.1 \%$ Tween-20, in order to anesthetized them. The nuclei of worms, including nuclei of gonads, were stained with DAPI (4',6-diamidino-2phenylindole) after permeating worms with Carnoy solution (60\% ethanol, $30 \%$ chloroform and $10 \%$ glacial acetic acid for 1 hour at $-20^{\circ} \mathrm{C}$ and washing three times with a phosphate saline buffer containing $0.1 \%$ tween-20 (PBS $0.1 \%$ Tween 20). DAPI was added at a final concentration of $50 \mu \mathrm{g} / \mathrm{mL}$. Worms were incubated for 30 minutes in darkness at room temperature. Finally, DAPI stained worms were put on a $2 \%$ agarose pad. Mounting oil vectashield was added, mantled with cover slips and sealed with polish nail. 


\subsubsection{Microscope images}

Nikon Eclipse 90i equipped with an ORCA ER camera (Hammatsu) and Olympus IX81 confocal spinning disk (Yokogawa CSU-X1 disk unit and equipped with a Photometrics Evolve EM-CCD camera) were used and managed through Metamorph software. Microscope Z-stack images were converted into a Z-projection of maximum intensities using ImageJ 1.47v. Zprojected images were mounted in Adobe Photoshop CS5 in gray-scale. When working simultaneously with Cherry and GFP, images were converted to RGB color in order to make merges with Photoshop.

\subsubsection{Statistical analysis}

Data were analyzed using Prism 5.0 and Microsoft Excel softwares.

\subsection{Description of the alleles constructed in this thesis}

\subsection{1. mes-4(sal25[K10A, E11A, N12A])}

The KEN-box motif of MES-4 protein was mutated to triple alanine (K10A, E11A, N12A) by CRISPR-Cas9. Microinjection mix consisted of preassembled gRNA-Cas9 complex complexes (Paix et al., 2015): $15 \mu \mathrm{M}$ tracRNA, $12.5 \mu \mathrm{M}$ of mes-4(aaa)sgRNA (from IDT), $0.2 \mu \mathrm{g} / \mu \mathrm{L}$ of mes4aaa mer (which incorporates mutations K10A, E11A, N12A) and $0.5 \mu \mathrm{g} / \mu \mathrm{l}$ Cas9 (from IDT). We also included in the mix the co-injection marker based on dpy-10(cn64)(Kim et al., 2014) : $2.5 \mu \mathrm{M}$ of sgRNAdpy-10 and $0.05 \mu \mathrm{g} / \mu \mathrm{L}$ of dpy-10 mer. After 3 to 4 days, Dpy and/or Rol worms were isolated. Insertion of KEN $\rightarrow$ AAA mutation was checked by PCR with the oligos mes-4aaa check 1 and mes-4aaa check 2 followed by digestion with Pst, since the mutated sequence included the recognition of this restriction enzyme. Mutant alleles yield two fragments of 600 and $400 \mathrm{bp}$ while the wild-type allele produces a single fragment of $1 \mathrm{kbp}$.

\subsection{2. mes-3(sal26[K37A, E38A, N39A])}

The KEN-Box motif of MES-3 protein was mutated into triple alanine (K37A, E38A, N39A) by CRISPR-Cas9. Microinjection mix consisted of preassembled gRNA-Cas9 complex complexes (Paix et al., 2015): $15 \mu \mathrm{M}$ tracRNA, 
$6.25 \mu \mathrm{M}$ of mes-3(aaa)sgRNA 1 (from IDT), $6.25 \mu \mathrm{M}$ of mes-3(aaa)sgRNA 2 (from IDT), $0.2 \mu \mathrm{g} / \mu \mathrm{L}$ of mes3aaa mer (which incorporates mutations K37A, E38A, N39A) and $0.5 \mu \mathrm{g} / \mu \mathrm{L}$ cas9 (from IDT). We also included in the mix the co-injection marker based on $d p y-10(c n 64)$ (Kim et al., 2014) :2.5 $\mu \mathrm{M}$ of sgRNAdpy-10 and $0.05 \mu \mathrm{g} / \mu \mathrm{l}$ of dpy-10(cn64) mer. After 3 to 4 days, Dpy and/or Rol worms were isolated. Mutations incorporate a Pstl site. Insertion of $\mathrm{KEN} \rightarrow$ AAA mutation was checked by PCR with the oligos mes-3aaa check 1 and mes-3aaa check 2 followed by digestion with Pstl, since the mutated sequence included the recognition of this restriction enzyme. Mutant alleles yield two fragments of 600 and 400 bp while the wild-type allele produces a single fragment of $1 \mathrm{kbp}$.

\subsection{3. mes-3(sal12[mes-3::GFP + loxP 3xmyc::let-858utr + sqt(d) + hs::Cre + hygR^3+ loxP Flag::mes-3UTR]) and mes-3(sal13[mes- 3::GFP + loxP Flag::mes-3UTR])}

We used the method described in (Dickinson et al., 2015) to label Cterminus of MES-3 with GFP. N2 were microinjected with a mix containing: 10 $\mathrm{ng} / \mu \mathrm{L}$ of repair template pmes-3::GFP^SEC, $50 \mathrm{ng} / \mu \mathrm{L}$ of psgRNAmes-3, 50 ng/ $\mu \mathrm{L}$ of peft-3::cas9-SV40_NLS::tbb-2utr and a cocktail of plasmids coding for extrachromosomal markers $(10 \mathrm{ng} / \mu \mathrm{L}$ of $\mathrm{pGH} 8,2.5 \mathrm{ng} / \mu \mathrm{L}$ of $\mathrm{pCFJ} 90$ and 5

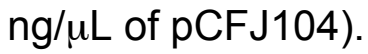

Repair template, pmes-3::GFP^SEC, was constructed as follows. First, 1 kbp-homology arms flanking stop codon of mes-3 were amplified by PCR with high fidelity polymerase Q5 and purified by gel. 5' homology arm was amplified with oligos 5 arm mes $3 \mathrm{fw}$ and 5 arm mes3 rev. 3' homology arm was amplified with oligos 3 arm mes $3 \mathrm{fw}$ and 3 arm mes 3 rev. On the other hand, plasmid pDD285 was digested with Avrll and Spel enzymes (Dickinson et al., 2015) and assembled with 5' and 3' homology arms to generate pmes-3::mkate^SEC. For reaction assembly, we used NEBuilder HiFi DNA assembly mix (from NEB). $5 \mu \mathrm{L}$ of assembly product was transformed into $E$. coli $\mathrm{DH} 5-\alpha$ and incubated overnight at $37^{\circ} \mathrm{C}$. Colonies transformed with desired pmes-3::mkate were analyzed with appropriated restriction enzymes 
Cas9-sgRNA target site was chosen as described in (Dickinson et al., 2015). Plasmid coding for guide RNA (gRNA) directed against the 3' end of mes-3 was obtained as follows. Two PCRs were carried out using in both cases pU6::unc-119_sgRNA as DNA template. For the first PCR we used oligos CRISP3 and sgRNA mes-3 rev, while in the second reaction oligos CRISP4 and sgRNA mes-3 dir. Both amplicons, $500 \mathrm{bp}$ and $350 \mathrm{bp}$, respectively, were gel purified in $50 \mu \mathrm{L}$ of final volume. Then, fragments were annealed by preparing a mix containing $40 \mu \mathrm{L}$ of each fragment, $20 \mu \mathrm{L}$ of buffer Q5, $2 \mu \mathrm{l}$ of dNTPs and 2 $\mu$ l of Q5. A 20 cycles-Q5-PCR program was run. The resulting fragment of 800 pb were purified by gel and inserted into pJET1.2, generating psgRNAmes-3 plasmid.

Microinjected worms were grown during 3 days at $25^{\circ} \mathrm{C}$. Then, hygromycin was added at a final concentration of $250 \mu \mathrm{g} / \mathrm{mL}$ per plate. After several days, we obtained hygromycin resistant worms that were roller (Rol) as well (phenotype included in hygro-resistant cassette (Dickinson et al., 2015)). We isolated 5 worms from every dish and checked out by PCR both extremes of the insertion. Left side was checked out with oligos 5 ext mes3 and GFP sec rev, while the right side with oligos Hygro 1 and 3 ext mes-3 (Fig 2.1). Cre-Lox induction upon $32^{\circ} \mathrm{C}$ heat-shock 2 hours allows the excision of the cassette, coupling GFP with the mes-3 3' UTR. We isolated 5 L1-L2 larvae, heat-shocked them and looked for among the F1 non-roller worms. Excision of the cassette was confirmed by PCR with oligos GFP Utr dir and 3 ext mes-3, which produce a fragment of $1.1 \mathrm{kpb}$ only when Lox recombination has taken place. 


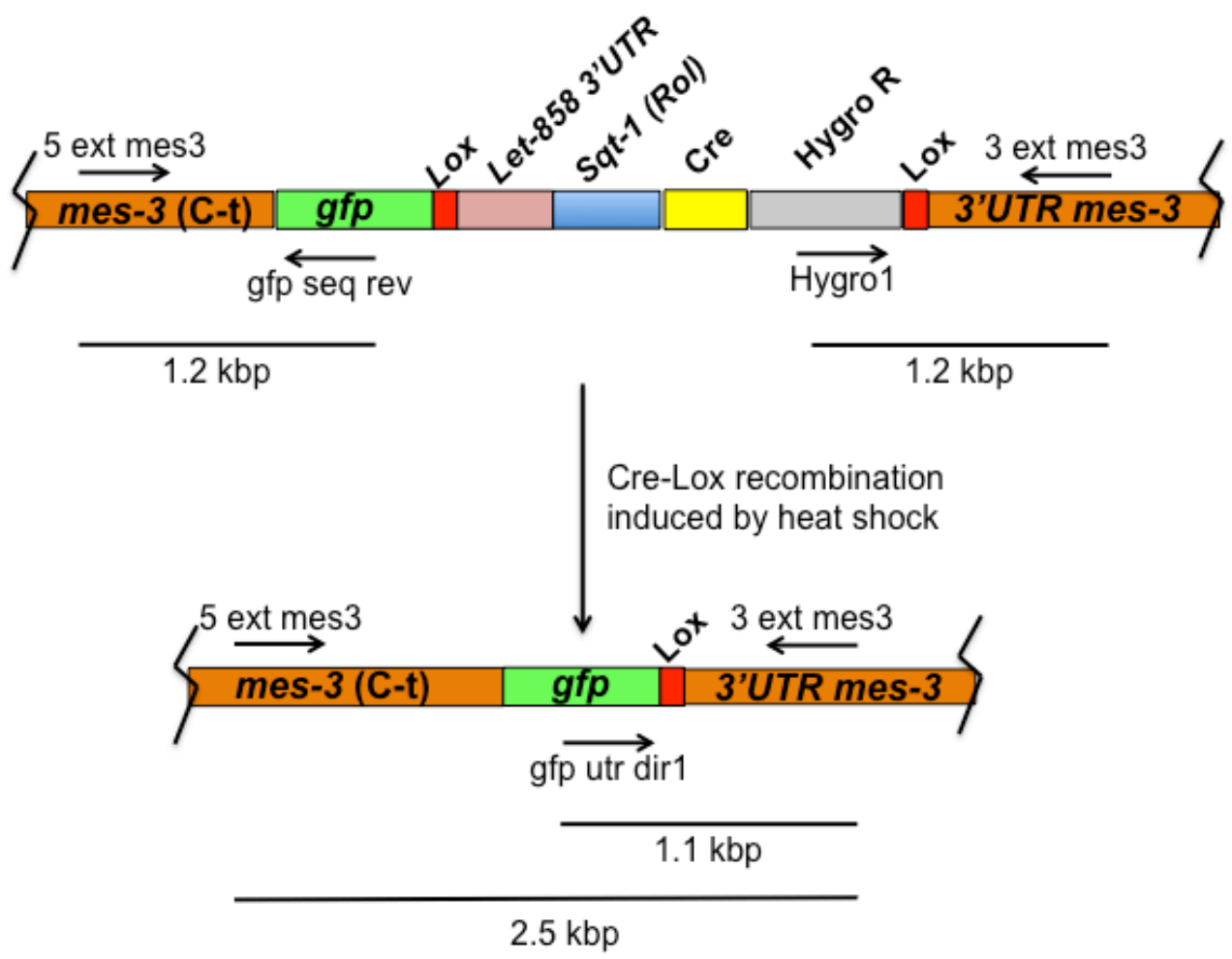

Figure 2.1. Insertion of GFP in mes-3 C-terminus (C-t). mes-3 was tagged at its C-t with GFP followed by an excisible cassette (Dickinson et al., 2015). This cassette codes for phenotypic markers Hygro $R$ and Sqt-1, which gives hygromycin resistance and roller (Rol) movility, respectively. Induction of Cre recombinase by heat shock leads to Lox sites recombination and cassette excision, leaving mes-3::GFP in frame with its own 3'UTR. Oligos 5 ext mes3 and gfp seq rev on one side and oligos Hygro1 and 3 ext mes3, on the other side, were used to check the left and right side of insertion, respectively. Cassette excision was confirmed with oligos 5 ext mes 3 and 3 ext mes 3 on one side, and oligos gfp utr dir 1 and 3 ext mes 3 , on the other side.

\subsection{4. mes-3(sal13[mes-3(K37A, E38A, N39A)::GFP^3xFlag::mes- 3UTR])}

This allele was constructed using the same method as the followed with mes-3(sal26) but using worms already carrying the mes-3(sal29) allele.

\subsection{5. mes-3(sal28[mes-3::tbb2UTR + unc-119 (+)]) I)}

We created a mes-3 allele under the control of $t b b-2$ 3'UTR by CRISPR. EG8080 strain was microinjected with a mix that contained an in vitro preassembled gRNA-Cas9 complex (Paix et al., 2015): $15 \mu \mathrm{M}$ tracRNA (from IDT), $15 \mu \mathrm{M}$ mes-3sgRNA (from IDT), $0.5 \mu \mathrm{g} / \mu \mathrm{L}$ cas9 (from IDT) and $0.1 \mu \mathrm{g} / \mu \mathrm{L}$ of repair template pmes-3tbb2utr(unc-119+). 


\subsection{6. mes-3(sal29[mes-3::GFP::tbb2UTR + unc-119 (+)]) I)}

mes-3 gene was C-t tagged with GFP and put under the control of heterologous tbb-2 3'UTR by means of CRISPR-Cas9. Universal MosSCI EG8080 strain was microinjected with a mix that contained an in vitro preassembled gRNA-Cas9 complex (Paix et al., 2015): $15 \mu \mathrm{M}$ tracRNA (from IDT), $15 \mu \mathrm{M}$ mes-3sgRNA (from IDT), $0.5 \mu \mathrm{g} / \mu \mathrm{L}$ cas9 (from IDT) and $0.1 \mu \mathrm{g} / \mu \mathrm{L}$ of repair template pmes-3GFPtbb2utr(unc-119+).

pmes-3GFPtbb2utr(unc-119+) was constructed as follows. Plasmids pGFP FRT Hygro and pmes-3cherry were digested with Sfil. Then, $3.8 \mathrm{kpb}$ fragment from pGFP FRT Hygro and $4.9 \mathrm{kpb}$ fragment from pmes-3 cherry were ligated, generating pmes-3GFPtbb2utr(hygro) plasmid. Next, hygromycinresistant cassette marker was changed by unc-119 (+) complementation marker.

\subsection{7. fzr-1 (sal19)}

A full-length deletion of the ORF of $f z r-1$ was achieved by CRISPR-Cas9. Microinjection mix consisted of pre-assembled gRNA-Cas 9 complex complexes (Paix et al., 2015): $15 \mu \mathrm{M}$ tracRNA, $6.25 \mu \mathrm{M}$ of fzr-1sgRNA 1 (from IDT), 6.25 $\mu \mathrm{M}$ of fzr-1sgRNA 2 (from IDT) and $0.5 \mu \mathrm{g} / \mu \mathrm{L}$ cas9 (from IDT). We also included in the mix the co-injection marker based on dpy-10(cn64) (Kim et al., 2014): $2.5 \mu \mathrm{M}$ of sgRNAdpy-10 and $0.05 \mu \mathrm{g} / \mu \mathrm{L}$ of dpy-10 mer. Repair template was not supply in order to activate the direct ligation of both extremes by Nonhomologous End Joining (NHEJ). Deletion of fzr-1 was checked by PCR with oligos check fzr1.1/check fzr1.2 and the PCR product obtained was sequenced. The mutation was maintained balanced with $m n / 1$.

\subsubsection{Construction and insertion of a fzr-1-expressing transgene}

A sequence containing $3.5 \mathrm{kbp}$ upstream of fzr-1 start codon (which includes fzr-1 promoter, Pfzr-1), fzr-1 ORF and $0.83 \mathrm{kbp}$ downstream stop codon (that codes for $f z r-1$ 3' UTR) were included in pMosll vector and named pMosPfzr-1::fzr-1::3'UTR fzr-1. This plasmid was obtained as follows. First, Pfzr-1 was amplified with Q5 polymerase using oligos prom fzr1 fw and prom fzr-1 rev, which include a Mscl site in the 5' end and an Ascl site in the 3' end. 
This 3,5 kbp fragment was inserted in pJET1.2. Then, promoter of mex-5 from pPmex-5::fzr-1::SL2:::cherry was interchanged by Pfzr-1 after double digestion with $\mathrm{Mscl}$ and Ascl, yielding pPfzr-1::fzr-1::SL2::cherry. Next, plasmids pPfzr1::fzr-1::SL2::cherry and pJET 2last exons fzr-1::3'UTR were digested with Afel and Sbfl, producing, respectively, a $12.4 \mathrm{kbp}$ and 1.4 fragments that were ligated, originating pMosPfzr-1::fzr-1::3'UTR fzr-1.

pMosPfzr-1::fzr-1::3'UTR fzr-1 was microinjected in Universal MosSCl strain EG8082 for insertion in chromosome V (Frøkjær-Jensen et al., 2012, Frøkjaer-Jensen et al., 2008). Microinjection mix included: $50 \mathrm{ng} / \mathrm{\mu L}$ of pMosPfzr-1::fzr-1::3'UTR fzr-1, $50 \mathrm{ng} / \mathrm{uL}$ of pCFJ601 (coding for transposase) and a cocktail of plasmids coding for extrachromosomal markers $(20 \mathrm{ng} / \mathrm{\mu L}$ of pGH8, $20 \mathrm{ng} / \mu \mathrm{L}$ of pMA122, $5 \mathrm{ng} / \mu \mathrm{L}$ of pCFJ90 and $10 \mathrm{ng} / \mu \mathrm{L}$ of pCFJ104). Non-unc worms were checked for transgene insertion using oligos MosLIN and MosIILout.

\subsubsection{Construction and insertion of an inducible fzr-1-expressing transgene based on ribozyme-tetracycline system (fzr-1::rbz)}

We made use of a previously reported system for inducible gene expression (Wurmthaler et al., 2019). Briefly, this system consists of a ribozyme sequence inserted downstream in frame with the gene of interest. An unstable mRNA is produced due to self-cleavage of ribozyme, precluding its translation. After tetracycline addition, ribozyme activity is blocked, generating a stable mRNA that is translated (Fig 2.2). Ribozyme sequence was inserted into plasmid pMosPfzr-1::fzr-1::3'UTR fzr-1. Two DNA fragments of $0.84 \mathrm{kbp}$ and $0.99 \mathrm{kbp}$ were obtained after amplifications with oligo pairs RBZ-1 and RBZ-2, and RBZ-3 and RBZ-4, respectively. DNA template was extracted from JPM139 strain using Q5 polymerase. Oligos RBZ-2 and RBZ-3 include the sequence of the ribozyme. Both PCR-amplifed fragments were annealed. The resulting 1,78 kbp fragment was cloned into pJET1.2 and named pJET fzr-1 rbz. Ribozyme was inserted into plasmid pMosPfzr-1::fzr-1::3'UTR fzr-1 after double digestion with Afel and Spel both, pMosPfzr-1::fzr-1::3'UTR fzr-1 and pJET fzr-1 rbz. Resulting plasmid was named pMosPfzr-1::fzr-1::rbz::3’UTR fzr-1. 
pMosPfzr-1::fzr-1::rbz::3'UTR fzr-1 was microinjected in Universal MosSCI strain EG8081 for insertion in chromosome IV (Frøkjær-Jensen et al., 2012, Frøkjaer-Jensen et al., 2008). Microinjection mix included: $50 \mathrm{ng} / \mu \mathrm{L}$ of pMosPfzr-1::fzr-1::3'UTR fzr-1, $50 \mathrm{ng} / \mathrm{uL}$ of pCFJ601 (coding for transposase) and a a cocktail of plasmids coding for extrachromosomal markers (20 ng/uL of pGH8, $20 \mathrm{ng} / \mu \mathrm{L}$ of pMA122, $5 \mathrm{ng} / \mathrm{uL}$ of pCFJ90 and $10 \mathrm{ng} / \mathrm{uL}$ of pCFJ104). Non-unc worms were checked for transgene insertion using oligos MosRIN and MosIIRout.

\subsubsection{Construction and insertion of an inducible putative phospho-} null fzr-1-expressing transgene based on ribozyme-tetracycline system (fzr-1 $\left.{ }^{8}\right):$ rbz)

We ordered a synthetic DNA coding for a putative phospho-null version of $f z r-1, f z r-1(8 A)$. In this version of $f z r-1$, all sites phosphorylated by cdk/cyclin described so far, were mutated into alanines (8A) (The et al., 2015) (Fig 2.2). First, $f z r-1^{8 A}$ was inserted into plasmid pMosPfzr-1::fzr-1::3'UTR fzr-1 after double digestion with Ascl and Afel both, pMosPfzr-1::fzr-1::3'UTR fzr-1 and $f z r-1^{8 A}$, obtaining pMosPfzr-1::fzr-1(8A)::3'UTR fzr-1. Next, ribozyme sequence was inserted into plasmid pMosPfzr-1:: fzr-1(8A)::3'UTR fzr-1 after double digestion with Afel and Spel both, pMosPfzr-1:: fzr-1(8A):: $3^{\prime} U T R$ fzr-1 and pJET fzr-1 rbz. Resulting plasmid was named pMosPfzr-1:: fzr1(8A)::rbz::3'UTR fzr-1.

pMosPfzr-1::fzr-1::rbz::3'UTR fzr-1 was microinjected in Universal MosSCI strain EG8081 for insertion in chromosome IV like in previous sections. 
A

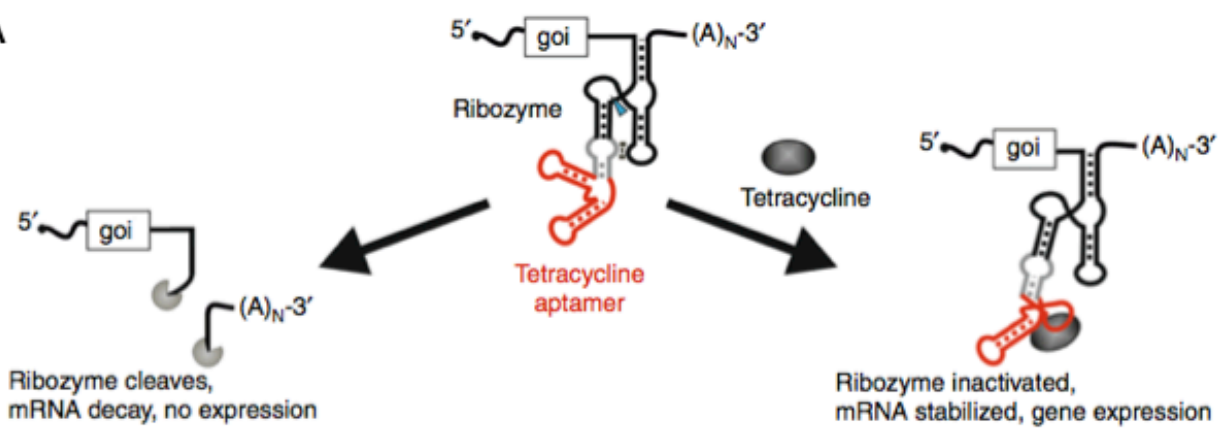

B

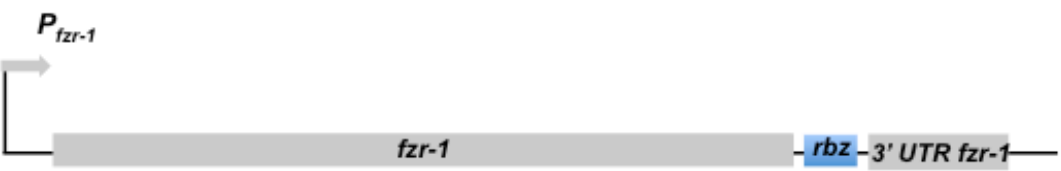

C

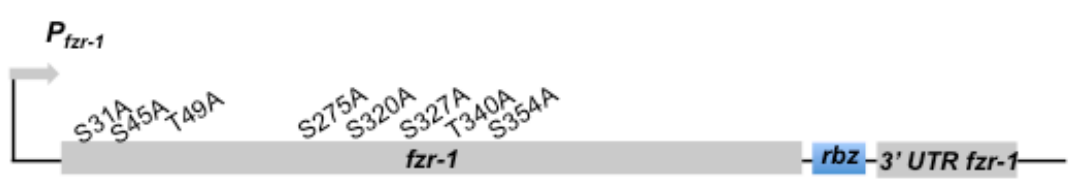

Figure 2.2. Inducible expression of $f z r-1$ and putative phospho-null $f z r-1$ transgenes with ribozyme-tetracycline system. A. Inducible expression of a gene of interest (goi) by a tetracycline-dependent ribozyme switch (Wurmthaler et al., 2019). A ribozyme coding sequence is located between goi ORF and 3' UTR. In absence of tetracycline, goi is transcribed followed by ribozyme, which self-cleavages, generating an unstable mRNA, which is not translated. Tetracycline inactivates ribozyme and allows goi to be expressed. B. Ribozyme-tetracycline system was adopted to generate an inducible $f z r-1$ transgene. C. Putative phospho-null fzr-1 transgene under the control of ribozyme-tetracycline system. Sites phosphorylated by cdk/cyclins along FRZ-1 were described in (The et al., 2015).

\subsubsection{Bipartite transgenic system used for marking somatic gonad} cells

Plasmids pMosPhlh-12cre pMosPlin-32cre were inserted in EG8080 Universal Mos strain and pMosPceh-22cre in Universal Mos strain EG8082. On the other hand, plasmid pMosPrps27::/ox::hygro::Iox::cherry was inserted in Universal Mos strain EG8081. Bipartite transgenic system works as indicate in figure 2.3. 


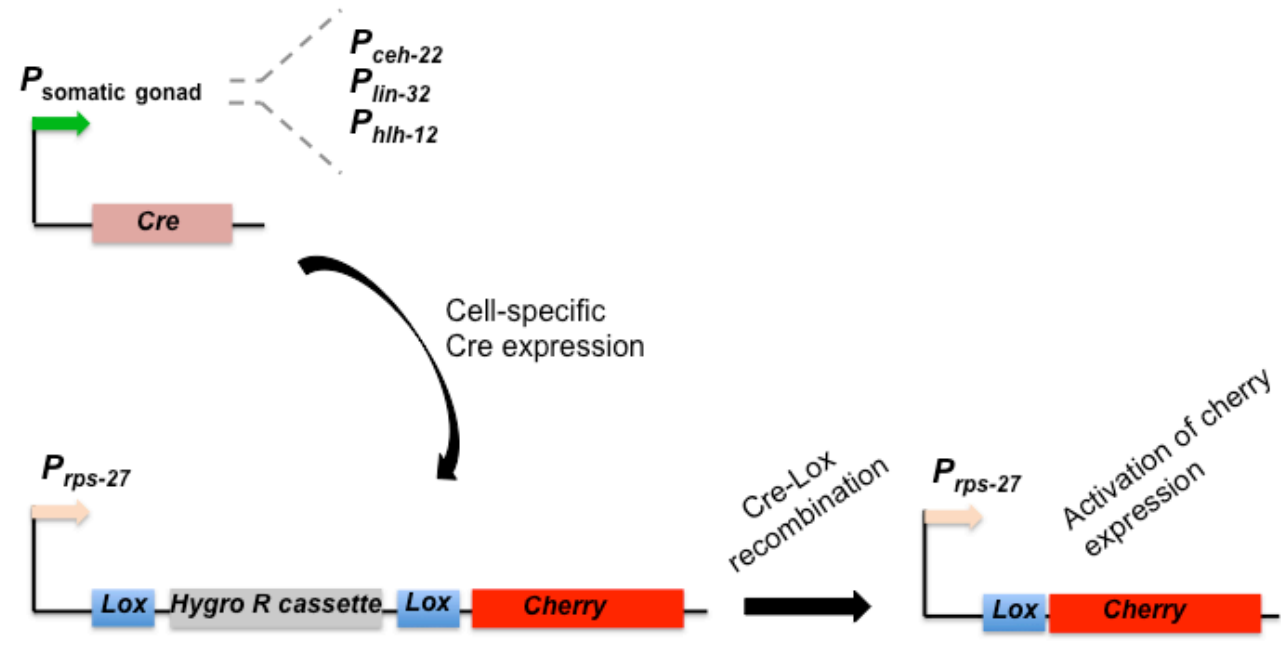

Figure 2.3. Bipartite transgenic system used for marking somatic gonad cells. The first part of the system consists of a Cre recombinase under the control of promoters that are expressed in somatic gonad cells: Pceh-22, Plin-32 and Phlh-12. Once activated, Cre activates recombination of Lox sites in the second part of this bipartite system. After lox recombination, Hygro $R$ cassette is eliminated, leaving Cherry in frame with housekeeping promoter Prps-27. Thereby, Cherry is expressed specifically in some cells of somatic gonad.

\subsubsection{C. elegans strains and oligonucleotides used}

Table 3. C. elegans strains

\begin{tabular}{ccc}
\hline Strain & Genotype & Origin \\
\hline CB1423 & him-6 & CGC \\
\hline DG1575 & tnls6 [lim-7::GFP + rol-6(su1006)] & CGC \\
\hline EG8080 & oxTi444 unc-1198ed3) III & CGC \\
\hline EG8081 & unc-119(ed3) III; oxTi177 IV & CGC \\
\hline EG8082 & unc-119(ed3) III; oxTi365 V & CGC \\
\hline JK2049 & qls19 [lag-2p::GFP::unc-54 3'UTR + rol-6(su1006)] V & CGC \\
\hline JPM36 & gld-3(q730) nos-3(q650)/mln1[mls14 dpy-10(e128)] & JK3182, NL2098 \\
\hline JPM42 & pgl-1(sal1[pgl-1::GFP + FRT hpt FRT]) & JPM21 \\
\hline JPM54 & lin-35(n4760)/hT2g l;/lI & MT15488, JK3025 \\
\hline JPM76 & mes-4(sal9[mes-4::mGFP::mes-4 utr]) V & JPM75 \\
\hline JPM78 & mes-4(sal11[mes-4(K10A, E11A, N12A)::mGFP::mes- & JPM77 \\
\hline
\end{tabular}


Table 3. C. elegans strains (cont)

\begin{tabular}{|c|c|c|}
\hline Strain & Genotype & Origin \\
\hline JPM93 & $\begin{array}{c}\text { mes-3(sal12[mes-3::GFP^3xmyc::let-858utr + sqt(d) + } \\
\text { hs::Cre + hygR^3xFlag:::mes-3UTR]) I }\end{array}$ & CRISPR in N2 \\
\hline JPM117 & $f z r-1(s a l 19) / m \ln 1[\mathrm{~m} / \mathrm{s} 14 d p y-10(\mathrm{e} 128)] \mathrm{II}$ & CRISPR in N2 \\
\hline JPM118 & $m \ln 1[m / s 14 d p y-10(e 128)] I I /+$ & N2, JPM36 \\
\hline JPM122 & $\begin{array}{c}\text { fzr-1 (sal19)/ mln1[m/s14 dpy-10(e128)] Il; pgl- } \\
\text { 1(sal1[pgl-1::GFP + FRT hpt FRT]) IV }\end{array}$ & JPM42, JPM117 \\
\hline JPM129 & $\begin{array}{c}\text { mes-3(sal15[mes-3(K37A, E38A, } \\
\text { N39A)::GFP^3xFlag::mes-3UTR]) I }\end{array}$ & CRISPR in JPM135 \\
\hline JPM131 & $\begin{array}{c}f z r-1 \text { (sal19)/ mln1[m/s14 dpy-10(e128)] Il; qls19 [lag- } \\
\text { 2p::GFP::unc-54 3'UTR + rol-6(su1006)] V }\end{array}$ & JK2049, JPM117 \\
\hline JPM132 & $\begin{array}{c}f z r-1(s a / 19) / m \ln 1[m / s 14 d p y-10(e 128)] ~ I l ; ~ r d l s 2[e h n- \\
3 a:: G F P+\text { rol-6(su1006)] V }\end{array}$ & RA45, JPM117 \\
\hline JPM135 & mes-3(sal13[mes-3::GFP^3xFlag::mes-3UTR]) & JPM93 \\
\hline JPM139 & salSi43 [Pfzr1::fzr1::fzr1UTR + unc-119(+)] V & MOS in EG8082 \\
\hline JPM143 & $\begin{array}{c}\text { fzr-1 (sal19)/ mln1[m/s14 dpy-10(e128)] II; salSi43 } \\
{[\text { Pfzr1::fzr1::fzr1UTR + unc-119(+)] V }}\end{array}$ & JPM117, JPM139 \\
\hline JPM145 & $\begin{array}{l}f z r-1(s a l 19) / \operatorname{mln} 1[\mathrm{mls} 14 \text { dpy-10(e128)] II; tnls6 [lim- } \\
\text { 7::GFP + rol-6(su1006)]. }\end{array}$ & DG1575, JPM117 \\
\hline JPM148 & $\begin{array}{c}\text { mes-3(sal15[mes-3(K37A, E38A, } \\
\text { N39A)::GFP^3xFlag::mes-3UTR]); mes-4(sal11[mes- }\end{array}$ & JPM78, JPM129 \\
\hline JPM157 & fzr-1(sal19) /mIn1 (II); him-5(e1490) (V) & JK3437, JPM117 \\
\hline JPM160 & $\begin{array}{c}\text { SalSi44 (Pfzr1::fzr-1::RBZ::fzr1UTR + unc-119(+)) IV; } \\
\text { qls19 [lag-2p::GFP::unc-54 3'UTR + rol-6(su1006)] }\end{array}$ & JK2049, \\
\hline JPM165 & $\begin{array}{c}f z r-1(\mathrm{sal19}) / \mathrm{m} \ln 1[\mathrm{~m} / \mathrm{s} 14 \text { dpy-10(e128)] II; wls78 } \\
{[S C M p:: G F P+\text { ajm-1::GFP + F58E10 (cosmid) + unc- }}\end{array}$ & RG733, JPM117 \\
\hline JPM166 & $\begin{array}{l}\text { fzr-1 (sal19)/ mln1[m/s14 dpy-10(e128)] II; SalSi44 } \\
\text { (Pfzr1::fzr1::RBZ::fzr1UTR + unc-119(+)) IV }\end{array}$ & JPM117, JPM161 \\
\hline JPM171 & him-5 (V) & N2, JPM157 \\
\hline JPM172 & $\begin{array}{c}f z r-1 \text { (sal19)/ mln1[m/s14 dpy-10(e128)] II; qls19 [lag- } \\
\text { 2p::GFP::unc-54 3'UTR + rol-6(su1006)] V; him-6 IV }\end{array}$ & CB1423, JPM131 \\
\hline JPM173 & $\begin{array}{c}\text { qIs19 [lag-2p::GFP::unc-54 3'UTR + rol-6(su1006)] V; } \\
\text { him-6 IV }\end{array}$ & CB1423, JPM131 \\
\hline JPM174 & $\begin{array}{c}f z r-1(\text { sal19) /mln1 (II); ccls444 [arg-1::GFP + dpy- } \\
20(+)] I V\end{array}$ & JPM157, PD4443 \\
\hline JPM175 & $\begin{array}{c}f z r-1(\text { sal19) /mln1 (II); him-5(e1490) (V); ccls444 [arg- } \\
1:: G F P+d p y-20(+)] \text { IV }\end{array}$ & JPM157, PD4443 \\
\hline JPM176 & $\operatorname{him}-5(\mathrm{e} 1490)(V) ; c c / s 444[\arg -1:: G F P+d p y-20(+)] I V$ & JPM157, PD4443 \\
\hline JPM177 & $\begin{array}{l}f z r-1(\mathrm{sal19}) / \mathrm{mln} 1[\mathrm{~m} / \mathrm{s} 14 d p y-10(\mathrm{e} 128)] \mathrm{II} ; \mathrm{him}- \\
\text { 8(e1489) IV; ezls1 [K09C8.2::GFP + pRF4]X }\end{array}$ & JPM117, DZ224 \\
\hline JPM179 & salSi45 (Hyg Pfzr1::fzr1(8A)::RBZ::fzr1 UTR) IV & MOS in EG8081 \\
\hline JPM180 & $\begin{array}{c}f z r-1 \text { (sal19)/ mln1[m/s14 dpy-10(e128)] II; salSi45 } \\
\text { (Hyg Pfzr1::fzr1(8A)::RBZ::fzr1 UTR) IV }\end{array}$ & JPM117, JPM179 \\
\hline
\end{tabular}


Table 3. C. elegans strains (cont)

\begin{tabular}{|c|c|c|}
\hline Strain & Genotype & Origin \\
\hline JPM181 & $\begin{array}{c}\text { salSi45 (Hyg Pfzr1::fzr1(8A)::RBZ::fzr1 UTR) IV; } \\
\text { qls19 [lag-2p::GFP::unc-54 3'UTR + rol-6(su1006)] V }\end{array}$ & JK2049, JPM179 \\
\hline JPM182 & SalSi46 [Prps27::lox::hygro::lox::cherry] IV & MOS in EG8081 \\
\hline JPM183 & $\begin{array}{c}\text { fzr-1(ku298) unc-4(e120) II; mes-4(sal9[mes- } \\
4:: m G F P:: m e s-4 \text { utr]) V }\end{array}$ & MH1829, JPM76 \\
\hline JPM185 & SalSi44 [Pfzr-1::fzr-1::RBZ::fzr-1UTR + unc-119(+)] IV & MOS in EG8081 \\
\hline JPM188 & $\begin{array}{l}f z r-1 \text { (sal19)/ mln1[mls14 dpy-10(e128)] II; SalSi44 } \\
\text { (Pfzr1::fzr1::RBZ::fzr1UTR + unc-119(+)) IV; qls19 }\end{array}$ & JPM166, JPM173 \\
\hline JPM189 & $\begin{array}{c}\text { salSi46[Prps27::Iox::hygro::Iox::cherry] IV; salSi47 } \\
\text { [Pceh22::cre] V }\end{array}$ & JPM204, JPM182 \\
\hline JPM191 & $\begin{array}{c}\text { mes-3(sal13[mes-3::GFP^3xFlag::mes-3UTR]) I; fzr- } \\
\text { 1(ku298) unc-4(e120) II }\end{array}$ & JPM135, MH1829 \\
\hline JPM196 & $\begin{array}{l}\text { fzr-1 (sal19)/ mIn1[m/s14 dpy-10(e128)] II; salSi45 } \\
\text { (Hyg Pfzr1::fzr1(8A)::RBZ::fzr1 UTR) IV; qls19 [lag- }\end{array}$ & JPM173, JPM180 \\
\hline JPM197 & mes-4(sal25[mes-4(K10A, E11A, N12A)]) & CRISPR in N2 \\
\hline JPM198 & $\begin{array}{l}\text { mes-3(sal26[mes-3(K37A, E38A, N39A)]) I, mes- } \\
\text { 4(sal11[mes-4(K10A, E11A, N12A)::mGFP::mes-4 }\end{array}$ & JPM78, JPM228 \\
\hline JPM204 & SalSi47 [Pceh22::cre] V & MOS in EG8082 \\
\hline JPM205 & salSi48[Phlh12::cre] III & MOS in EG8080 \\
\hline JPM206 & SalSi49[Plin32::cre] III & MOS in EG8080 \\
\hline JPM207 & $\begin{array}{l}\text { SalSi49[Plin32::cre] III; SalSi46 } \\
\text { [Prps27::lox::hygro::lox::cherry] IV }\end{array}$ & JPM182, JPM206 \\
\hline JPM208 & $\begin{array}{c}\text { SalSi48[PhIh12::cre] III; SalSi46 } \\
\text { [Prps27::Iox::hygro::lox::cherry] IV }\end{array}$ & JPM182, JPM205 \\
\hline JPM209 & SalSi50 [Pfzr1::fzr1(8A)::RBZ::fzr1 UTR] V & MOS in EG8082 \\
\hline JPM210 & $\begin{array}{l}\text { fzr-1(sal19) /mIn1 (II); SalSi48[Phlh12::cre] III; } \\
\text { SalSi46 [Prps27::lox:::hygro::lox::cherry] IV }\end{array}$ & JPM157, JPM208 \\
\hline JPM213 & $\begin{array}{c}\text { qls19 [lag-2p::GFP::unc-54 3'UTR + rol-6(su1006)] V; } \\
\text { syls50 [cdh-3::GFP + dpy-20(+) }\end{array}$ & JPM181, JPM149 \\
\hline JPM214 & $\begin{array}{l}\text { salSi45 (Hyg Pfzr1::fzr1(8A)::RBZ::fzr1 UTR) IV; } \\
\text { qls19 [lag-2p::GFP::unc-54 3'UTR + rol-6(su1006)] V; }\end{array}$ & JPM181, JPM149 \\
\hline JPM221 & $\begin{array}{c}\text { fzr-1(sal19)/ mln1[m/s14 dpy-10(e128)] II; tnls6 [lim- } \\
\text { 7::GFP + rol-6(su1006)]; SalSi48[Phlh12::cre] III; }\end{array}$ & JPM210, JPM145 \\
\hline JPM222 & $\begin{array}{c}\text { fzr-1 (sal19)/ mln1[m/s14 dpy-10(e128)] II; } \\
\text { SalSi48[Phlh12::cre] III; SalSi46 }\end{array}$ & JPM210, JPM131 \\
\hline JPM224 & SalSi51(Prps27::lox::hygro::lox::fzr-1) IV & MOS in EG8091 \\
\hline JPM225 & mes-3(sal28[mes-3::tbb-2 3'UTR + unc-119 (+)] I & CRISPR IN EG8080 \\
\hline JPM226 & mes-3(sal29[mes-3::GFP::tbb-2 3'UTR + unc-119 (+)] & CRISPR in EG8080 \\
\hline JPM228 & mes-3(sal26[mes-3(K37A, E38A, N39A)]) I & CRISPR in N2 \\
\hline JPM229 & $\begin{array}{c}\text { SalSi48[Phlh12::cre] III; SalSi46 } \\
\text { [Prps27::lox::hygro::/ox::cherry] IV; qls19 [lag- } \\
\text { 2p::GFP::unc-54 3'UTR + rol-6(su1006)] V }\end{array}$ & JPM208, JPM188 \\
\hline
\end{tabular}


Table 3. C. elegans strains (cont)

\begin{tabular}{|c|c|c|}
\hline Strain & Genotype & Origin \\
\hline JPM229 & $\begin{array}{c}\text { SalSi48[PhIh12::cre] III; SalSi46 } \\
\text { [Prps27::lox::hygro::lox::cherry] IV; qls19 [lag- }\end{array}$ & JPM208, JPM188 \\
\hline JPM231 & $\begin{array}{c}\text { mes-3(sa/30[mes-3(K37A, E38A, N39A)::GFP ::tbb-2 } \\
\text { 3'UTR + unc-119 (+)] I }\end{array}$ & CRISPR in JPM226 \\
\hline JPM233 & SalSi52 [Psth-1::GFP + unc-119 (+)] V & MOS in EG8002 \\
\hline JPM240 & $\begin{array}{c}\text { mes-3(sal26[mes-3(K37A, E38A, N39A)]) I; lin- } \\
\text { 15B(n744) X }\end{array}$ & MT2495, JPM228 \\
\hline JPM241 & $\begin{array}{c}\text { mes-4(sal25[mes-4(K10A, E11A, N12A)]) V; lin- } \\
15 B(n 744) X\end{array}$ & MT2495, JPM197 \\
\hline JPM242 & $\begin{array}{c}\text { mes-3(sal26[mes-3(K37A, E38A, N39A)]); mes- } \\
\text { 4(sal25[mes-4(K10A, E11A, N12A)]); lin-15B (n744) X }\end{array}$ & MT2495 \\
\hline JPM246 & $\begin{array}{c}\text { mes-3(sal31[mes-3(K37A, E38A, N39A)::tbb-2 3'UTR } \\
+ \text { unc-119(+)] I }\end{array}$ & CRISPR in JPM225 \\
\hline JPM247 & $\begin{array}{c}\text { mes-3(sal26[mes-3(K37A, E38A, N39A)]) l; mes- } \\
\text { 4(sal25[mes-4(K10A, E11A, N12A)]) V } \\
\end{array}$ & JPM197, JPM228 \\
\hline JPM248 & $\begin{array}{c}\text { fzr-1(sal19) /mIn1 (II); SalSi48[Phlh12::cre] III; } \\
\text { SalSi46 [Prps27::Iox::hygro::lox::cherry] IV; SalSi52 }\end{array}$ & JPM210, JPM233 \\
\hline JPM249 & $\begin{array}{c}\text { lin-35(n4760) l;mes-4(sal25[mes-4(K10A, E11A, } \\
\text { N12A)]) V }\end{array}$ & JPM54, JPM247 \\
\hline JPM250 & $\begin{array}{c}\text { mes-3(sal26[mes-3(K37A, E38A, N39A)]); lin- } \\
\text { 35(n4760) I }\end{array}$ & JPM54, JPM247 \\
\hline JPM251 & $\begin{array}{c}\text { mes-3(sal26[mes-3(K37A, E38A, N39A)]); lin- } \\
\text { 35(n4760) l; mes-4(sal25[mes-4(K10A, E11A, N12A)]) }\end{array}$ & JPM54, JPM247 \\
\hline MH1829 & $f z r-1(k u 298)$ unc-4(e120) II & CGC \\
\hline MT2495 & $\operatorname{lin}-15 B(n 744) X$ & CGC \\
\hline N2 & Wild-type Bristol & CGC \\
\hline PD4443 & ccls444 [arg-1::GFP + dpy-20(+)] IV & CGC \\
\hline RA45 & rdls2 [ehn-3a::GFP + rol-6(su1006)] V & CGC \\
\hline RG733 & $\begin{array}{c}\text { w/s78 [SCMp::GFP }+ \text { ajm-1::GFP + F58E10 (cosmid) } \\
+ \text { unc-119(+) IV }\end{array}$ & CGC \\
\hline
\end{tabular}


Table 5. Oligonucleotides

\begin{tabular}{|c|c|}
\hline Name & Sequence (5') \\
\hline 3 arm mes3 fw & CGTGATTACAAGGATGACGATGACAAGAGATGATTATCCCCTTTTTTCCTTGTATCTTT \\
\hline 3 arm mes3 rev & GGAAACAGCTATGACCATGTTATCGATTTCCGAATTGTCGAAAATTCGGAAATACGA \\
\hline 3 ext fzr1 & AACTTTTAACGGAAAATATTGGAACAG \\
\hline 3 ext mes3 & AGGGTTACTGTAGGATTACTGTAGTTTGGA \\
\hline 5 arm mes3 fw & ACGTTGTAAAACGACGGCCAGTCGCCGGCAACTCAAAAAATGTCCTGGAAGTTGCTG \\
\hline 5 arm mes3 rev & CATCGATGCTCCTGAGGCTCCCGATGCTCCATTAGAAGATGGTATATCTCGTTGGCG \\
\hline 5 ext FZR1 Nt & TTATCATGAATCTCTTGGTTTGAAGAG \\
\hline 5 ext mes3 & TAAAATTAATGTCAAAATATAGAAAAACAC \\
\hline CheckFZR1-1 & CTAGTGTCTCСTCTCCATTCTCTTAGCCGG \\
\hline CheckFZR1-2 & AGAGGCATAAATCGATCCCCGTAAGGATTC \\
\hline CheckFZR1-3 & GGGAGGTAATAATTTTCTTTAAAAAGGCAC \\
\hline CherryLOX-1 & TAGAATTCGGTACCATGGTCTCAAAGGGTGAAGAAGATAAC \\
\hline CherryLOX-2 & ATGGCCACTCAGGCCTGAGACTTTTTTCTTGGCGGCACAATA \\
\hline fzr1 cdk rev & AACTGTTCGACCTGTCGCCGCACCGAC \\
\hline GFP check lox & CAATCGGAGACGGACCAGTCCTCCTCCCAG \\
\hline gfp SEC rev & AGTGAACAATTCTTCTCСTTTACTCAT \\
\hline GFP utr DIR & GGAATCACCCACGGAATGGACGAGCTC \\
\hline GFP-lin REV & AGTGAAAAGTTCTTCTCCTTTACTCAT \\
\hline Hyg-3'UTR DIR & AACTGTTTCGCGGCCGCATTAATAGGC \\
\hline HYG-DIRECT & CCCAAAATCTACACAATGTTCTGTGTACAC \\
\hline HygLOX-1 & ATGGATCCATGAAAAAGCCTGAACTCACCGCGACG \\
\hline HygLOX-2 & ATGGTACCATAACTTCGTATAATGTATGCTATACGAAGTTATTAAAAAT \\
\hline Hygro 1 & GGATCCAATTACCTCTTCAACATCССТАCAT \\
\hline KEN box-1 & CTGACCAATTGCGAAGCG \\
\hline KEN box-2 & CCACCTGATGGATAACACGA \\
\hline Mes-4UTR & ATAGTACGATTAAAAAGAATGAGGGCACTG \\
\hline mes3 nested fw & TTGAATTGCCCATGGTTCGATATAAGCAAT \\
\hline mes3aaa check 1 & ATCGCGCCGAGACCCGCGTATTCTAAC \\
\hline mes3aaa check 2 & ATTCAAACAAACCGCATCGGTCTTTAG \\
\hline Mes4check-1 & CCAACGCTGCATCTTTCGTTCAAAAGTGT \\
\hline Mes4check-2 & ATAGTACGATTAAAAAGAATGAGGGCACTG \\
\hline MosL II OUT & GTTTACAGAAAGACATTTGAGAATGGC \\
\hline
\end{tabular}


Table 5. Oligonucleotides (cont)

\begin{tabular}{|c|c|}
\hline Name & Sequence $\left(5^{\prime}\right)$ \\
\hline MosL IN & ATAATAAACATTTTATCCGTTAACAAT \\
\hline $\mathrm{n} 4760 \mathrm{~F}$ & TCGACATCAAAATCCGGCTTTGTGAC \\
\hline $\mathrm{n} 4760 \mathrm{R}$ & ATACGTCTTAATGCGATTATAATTAAC \\
\hline oCF1491 (IV) & GTCACTCAAACCGATGCAGA \\
\hline oCF1492 (IV) & GCAATTTCGGCAATTTCAGT \\
\hline oCF1493 (II) & TGCTCGGAAGGACTTGATTT \\
\hline oCF1494 (II) & TTGCCACGTCTTCTTGAGTG \\
\hline oCF1501 (V) & ATGAAAGGCAAGCGTGAACT \\
\hline oCF1502 (V) & CCGTCGAAGACCCAATAAGA \\
\hline oCF1513 (III) & CAATTTCAGGGGCGAAAGTA \\
\hline oCF1514 (III) & GCGTTCCGTAAATTGGAAGA \\
\hline oCF1608 (I) & CAGCCTACGCCACTTACACA \\
\hline oCF1609 (I) & CCAATGGCTCCTGAAAAAGA \\
\hline Phlh12-1 & ACAGCTAGCTTTAGAACATTAAAAAAAACTAATA \\
\hline Phlh12-2 & TGTGGCGCGCCTTTAATAAAATTGTGTAAGATGACGCTA \\
\hline Plin32-1 & ACAGCTAGCCTAATCGGAACGGTGTCTCCAAACTTC \\
\hline Plin32-2 & TGTGGCGCGCCGGTTGGTCTGACTGAAAACGACGATGTG \\
\hline Psth1-1 & ATACCTGCAGGATTCAAGGGGTTGCTGTTGCTGTGGA \\
\hline Psth1-2 & TATGGCGCGCCGTTTGCTCTAGCACAAAAAGCATCACTT \\
\hline RBZ-5 & GGCGCGCCATAGGTACCATTTTCACATCAAACAAACAAAGGCGCGTC \\
\hline RBZFZR-4 & GATTACGCCAAGCTACGTAATACGACTCAC \\
\hline sgRNA mes-3 direct & GGGATAATCTAATTAGAAGAGTTTTAGAGCTAGAAATAGCAAGTTAA \\
\hline sgRNA mes-3 rev & TCTTCTAATTAGATTATCCCAAACATTTAGATTTGCAATTCAATTAT \\
\hline Utr mes3 check & AGAATTGCCACCCACCACGATTTGGTATAG \\
\hline
\end{tabular}


RESULTS 



\section{RESULTS}

\subsection{MES-3 is a target of APC/C $\mathrm{C}^{\mathrm{FZR}-1}$}

Previous work done in our laboratory described that MES-4 is a target of $\mathrm{APC} / \mathrm{C}^{\mathrm{FZR}-1}$, and that mutation of its sole KEN box abolished the ability of APC/C ${ }^{F Z R-1}$ to degrade the protein (Rivera-Martín S, 2018). In the germline, MES-4 is present at distal mitotic nuclei, barely detectable in the early-to midpachytene region, and up-regulated in later pachytene and oocytes (Fong et al., 2002). In the mutant allele lacking the KEN box (mes-4(AAA)), the MES-4::GFP signal appeared uniform throughout the germline. This distribution was also observed in fzr-1 silenced germlines and in gonads from fzr-1(ku298) worms (Fig. 3.1).
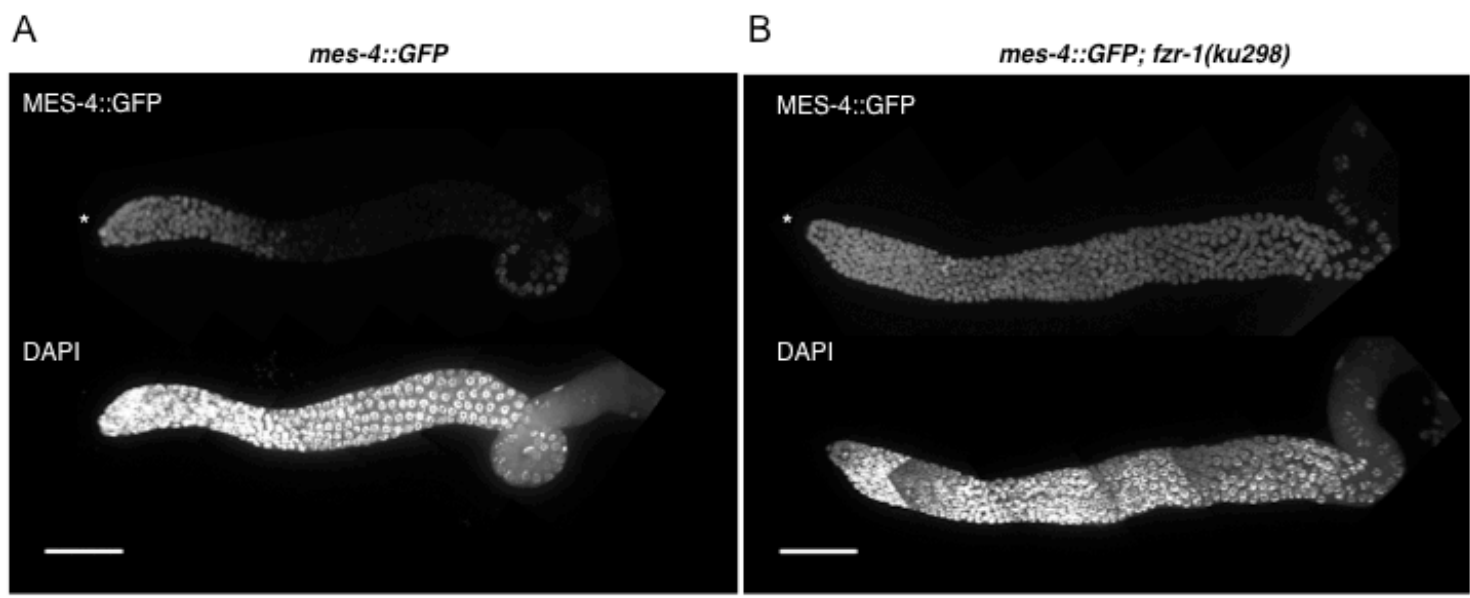

Figure 3.1. MES-4::GFP is expressed throughout the entire germline in fzr-1(ku298). A. Control germline expresses MES-4::GFP protein in distal an proximal region, while its levels drop in pachytene $(n=7)$. B. In germline of $f z r-1$ (ku298) mutants, MES-4 expression is observed throughout the entire germline $(n=9)$. DAPI-stained germlines are shown in every panel. Asterisks $\left(^{*}\right)$ indicate the distal tip of germline. Scale bar: $50 \mu \mathrm{m}$.

Strikingly, the absence of APC/C ${ }^{\mathrm{FZR}-1}$-mediated degradation of MES-4 seemed to have not a big impact on the fertility of $C$. elegans. We have analyzed the brood size of worms carrying KEN-less versions of MES-4 (tagged or not with GFP), and we observed a decrease of roughly $10 \%$ in fertility of both mes-4::GFP and mes-4(AAA) with respect to control N2 (Fig. 3.2). 


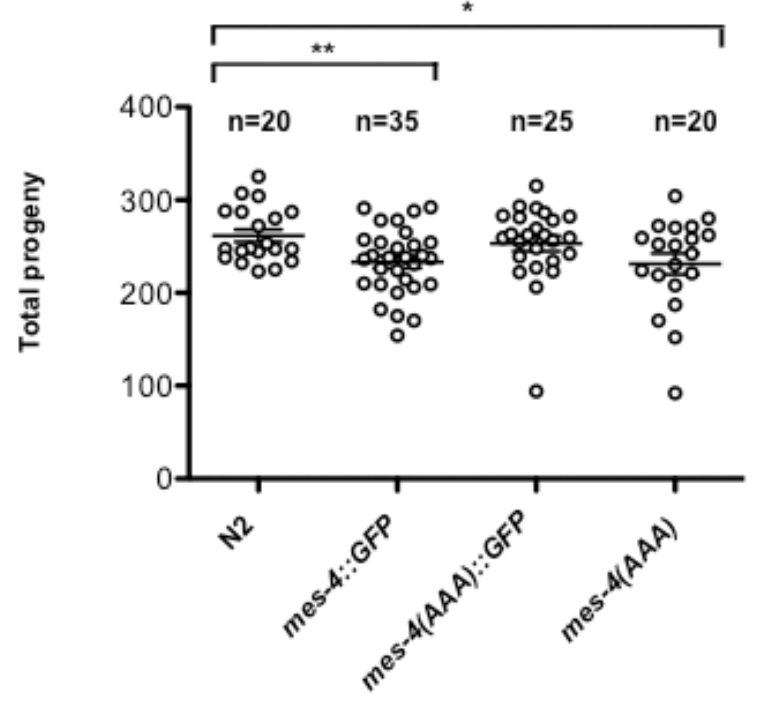

Figure 3.2. Mutation of KEN-Box motif of mes-4 has low impact on fertility. mes-4::GFP and mes$4(A A A)$ show a $10 \%$ decrease in fertility levels compared to $N 2$. However, mes-4(AAA)::GFP is as fertile as N2. Fertility assay were carried out at $20^{\circ} \mathrm{C}^{*}=\mathrm{p}$ value $<0.05 .{ }^{* *}=\mathrm{p}$ value $<0.01$.

These results suggested a minor role in the degradation of MES-4 by $\mathrm{APC} / \mathrm{C}^{\mathrm{FZR}-1}$. However, MES-4 plays a primordial role in the maintenance of germline (Garvin et al., 1998), and FZR-1 seems to have an essential role in the fertility of worms (Fay et al., 2002). These observations prompted us to wonder whether the mild effects in mes-4(AAA) could be caused by the existence of additional targets for $A P C / C^{F Z R-1}$ in the germline, which scheduled degradation by APC/C ${ }^{\text {FRR-1 }}$ compensated for the absence of MES-4 degradation.

We have searched for the presence of APC/C signatures (KEN and Destruction boxes) in proteins with regulatory roles in the germline, using the GPS-ARM algorithm (Liu et al., 2012). Appealingly, we have found in the amino acid sequence of MES-3 the presence of a KEN box (K37, E38, N39). MES-3 forms, together with MES-2 and MES-6, the $C$. elegans version of the widely conserved Polycomb repressive complex 2 (PRC2) (Bender et al., 2004b). Interestingly, PRC2 cooperated with MES-4 in the maintenance of the germline (Gaydos et al., 2012), abounding in our original idea of some genetic redundancy to explain the absence of effects in the mes-4 $4^{(A A A)}$ mutants.

To address if MES-3 is targeted by APC/C ${ }^{\text {FZR-1 }}$, we constructed by CRISPR-Cas9 a GFP tagged allele of MES-3 at its C-terminus. We first 
analyzed the distribution of the GFP signal through the gonad, and found that it was similar to the distribution described previously using immunostaining with anti-MES-3 antibodies: it was present in distal mitotic nuclei, undetectable in the pachytene region, and present in the proximal meiotic region (Fig. 3.3A). We have found that this allele behaves similarly to a wild-type allele concerning the fertility of the worms (Fig. 3.3B).
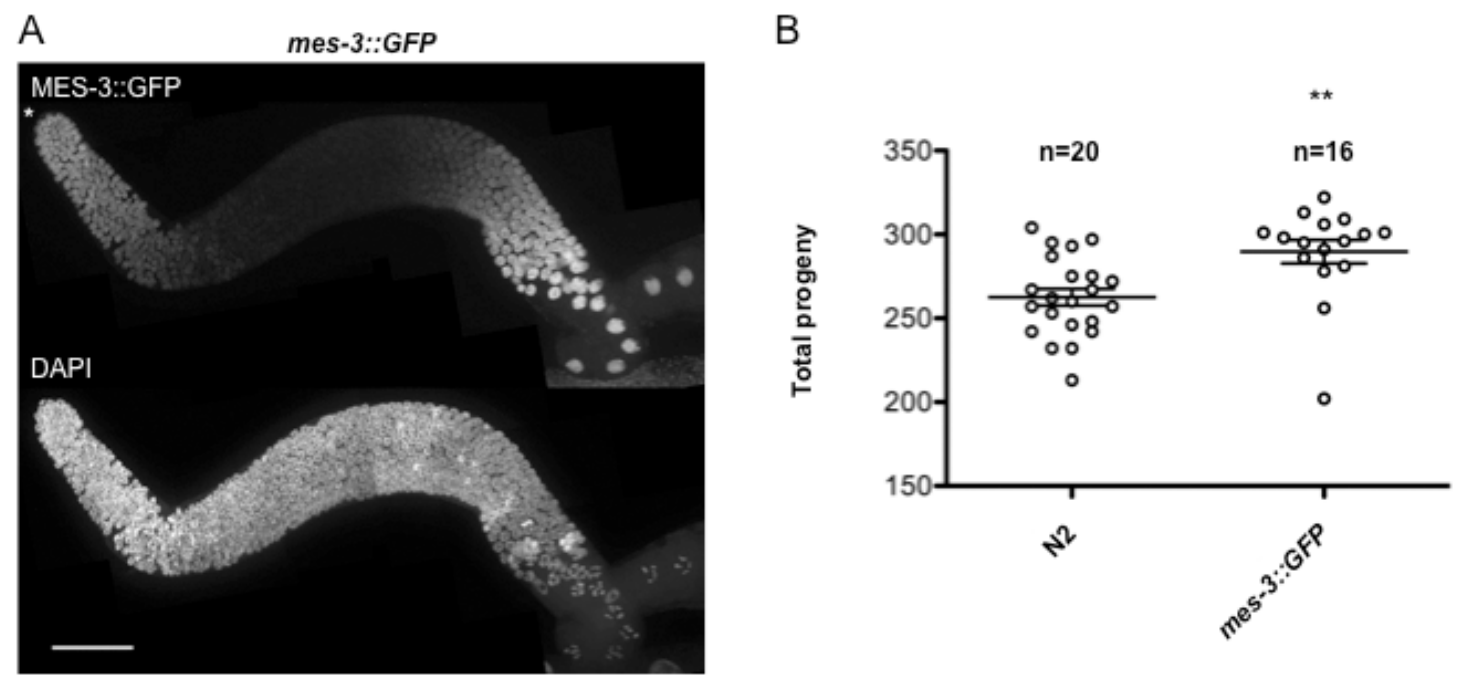

Figure 3.3. mes-3::GFP is a functional allele expressed in distal and proximal germline. A. Extruded and DAPI-stained germline showing MES-3::GFP expression in distal an proximal region. Previous results based on inmunohistochemistry also detected MES-3 in distal an proximal germline (Xu et al., 2001). Asterisks $\left(^{*}\right)$ indicate the distal tip of germline Scale bar: $50 \mu \mathrm{m}$ B. Allele mes-3::GFP is slightly, but significantly, more fertile than $\mathrm{N} 2 .{ }^{* *}=p$ value $<0.01$.

We have examined the distribution of the MES-3::GFP fusion protein in gonads from fzr-1(ku298) mutants and fzr-1 silenced worms (Fig. 3.4). Encouragingly, we have found that in these conditions, it was possible to observe the presence of GFP signal in the early pachytene region. This result suggests that similarly as we have described for MES-4, the distribution of MES-3 along the gonad could be controlled by APC/C $\mathrm{CZR}^{\mathrm{FR}-1}$. 
A

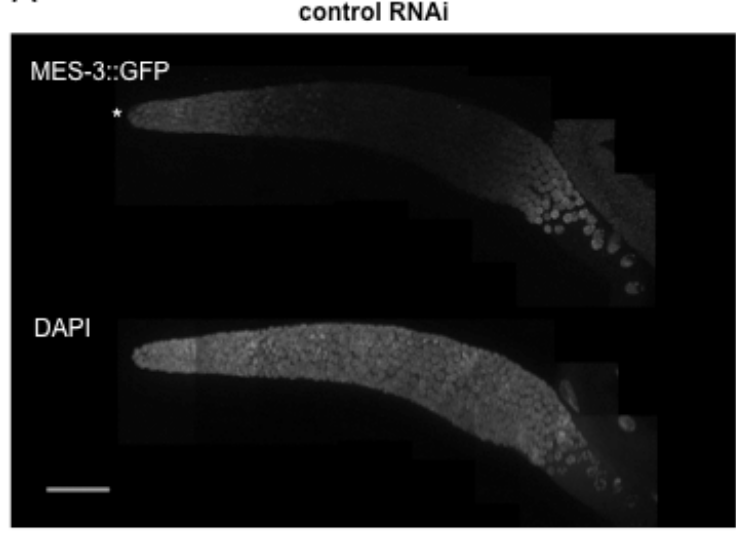

B

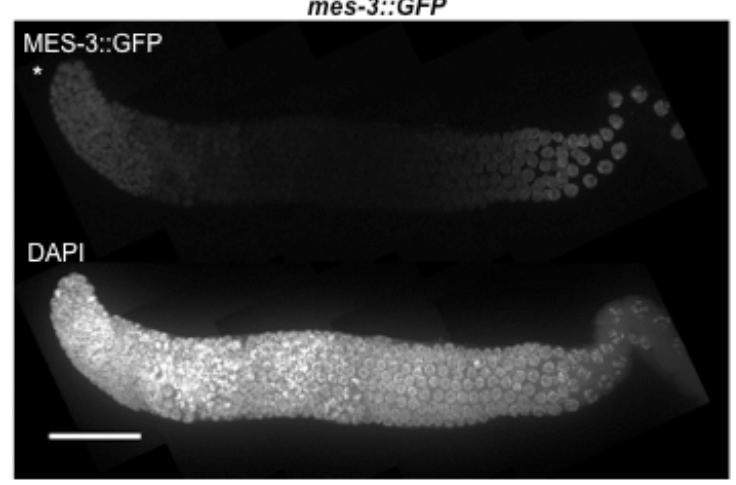

fzr-1 RNAi

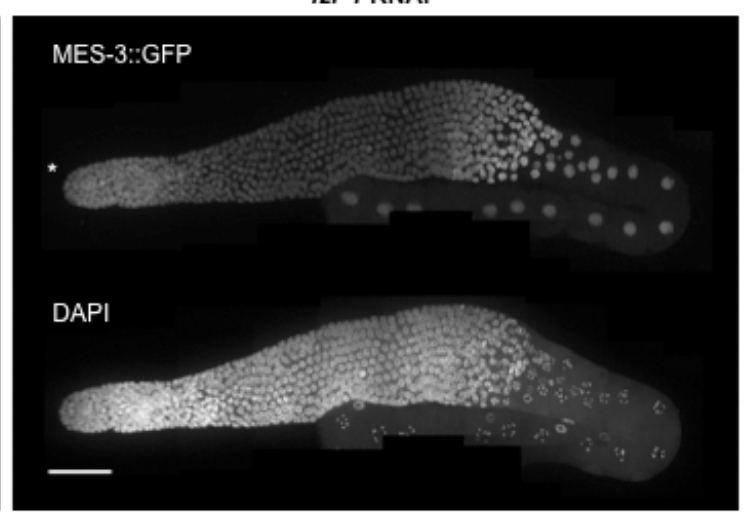

mes-3::GFP; fzr-1(ku298)

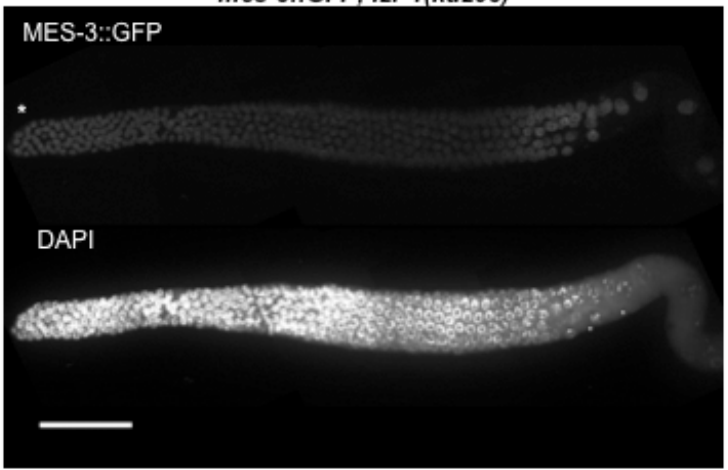

Figure 3.4. Polycomb subunit MES-3 invades pachytene region upon depletion of FZR-1 levels or activity. A. Control RNAi germlines express MES-3 protein in distal an proximal region, while its levels drop in pachytene $(n=9)$ (left panel). fzr-1 silenced worms express MES-3 throughout the entire germline $(n=9)$ (right panel). B. The same extension of MES-3 expression is observed in germlines of $f z r-1$ (ku298) mutants $(n=9)$. Control germline is shown in left panel $(n=9)$. DAPI stained germlines are shown in every panel. Asterisks $\left(^{*}\right)$ indicate the distal tip of germlines. Scale bar: $50 \mu \mathrm{m}$.

To determine whether MES-3 was a direct target of $A P C / C^{F Z R-1}$, we exchanged the amino acids composing the KEN-box with alanine residues at the endogenous mes-3 locus. In line with the assumption of MES-3 being a direct target of $A P C / C^{F Z R-1}$ we have found that the mutant allele lacking the KEN box (mes-3(AAA)) recapitulates the gonad distribution observed in $f z r-1$ silenced germlines as well as in fzr-1(ku298) (Fig. 3.5). 

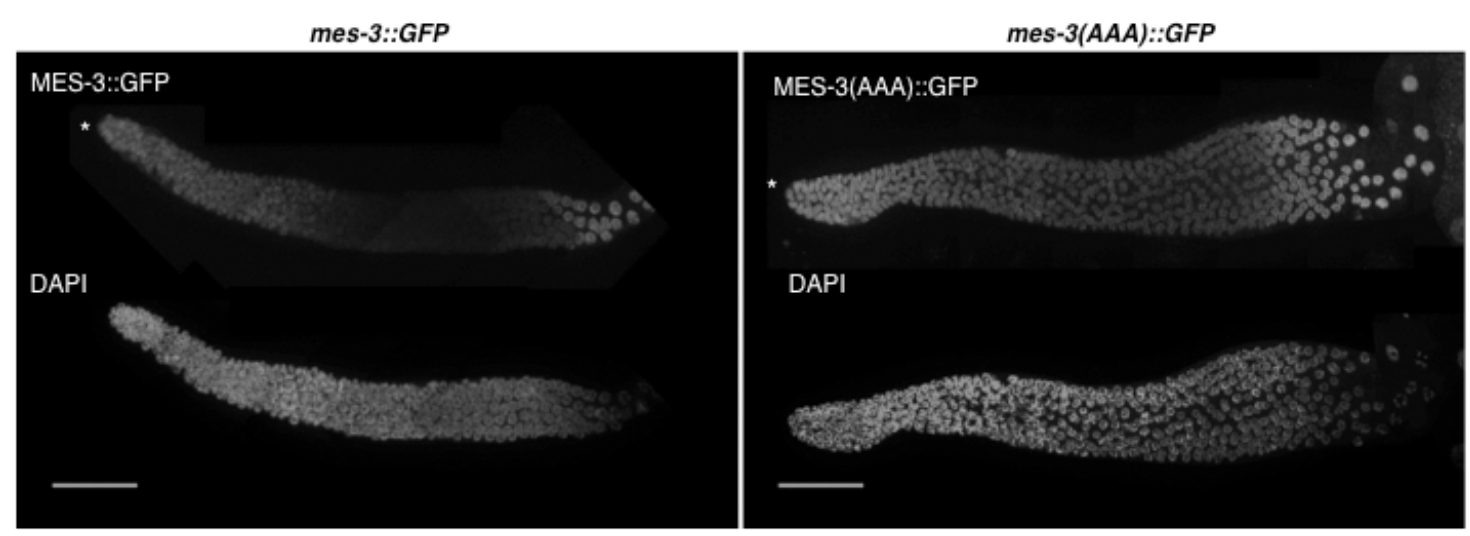

Figure 3.5. APC/C ${ }^{\text {FZR-1 }}$ targets MES-3 through its KEN-box. MES-3 contains a KEN-box motif in its Nterminal sequence, (K37, E38, N39), a motif recognized by APC/C $\mathrm{FZR}^{-1}$. Mutation of KEN-box motif into triple alanine (K37A, E38A, N39A) yields expression of MES-3 in the entire germline. Left panel shows expression of MES-3::GFP in germline of control strain $(n=13)$. Right panel shows expression through the germline of mes-3(AAA)::GFP $(n=10)$. Both panels also include DAPI-stained germlines. Asterisks $\left(^{*}\right)$ indicate the distal tip of germlines. Scale bar: $50 \mu \mathrm{m}$.

\section{2. $\mathrm{APC} / \mathrm{C}^{\mathrm{FZR}-1}$ and regulatory signals at the mes-3 3 'UTR collaborate to restrict the presence of MES-3 protein at early pachytene}

The absence of MES-3 at early pachytene stage was previously attributed to translational repression mediated by GLD-1, which recognizes mes-3 3' UTR (Xu et al., 2001). One appealing possibility is that the inhibition of mRNA translation collaborates with the APC/C $\mathrm{C}^{\mathrm{FR}-1}$-mediated degradation to maintain MES-3 out from early pachytene region. To address this possibility, we have constructed worms carrying an endogenous mes-3::GFP allele in which we exchanged its native 3'UTR with the 3'UTR from tbb-2 gene, which is considered an ubiquitous UTR and is not repressed by GLD-1 (Wright et al., 2011). We have also combined the mes-3(AAA) allele with the alternative $t b b-2$ 3' UTR.

We have observed that the levels of MES-3::GFP in the gonad when the mes-3 mRNA was controlled by tbb-2 3'UTR were lower than those observed when the mRNA carried the native mes-3 3'UTR (Fig 3.6). 


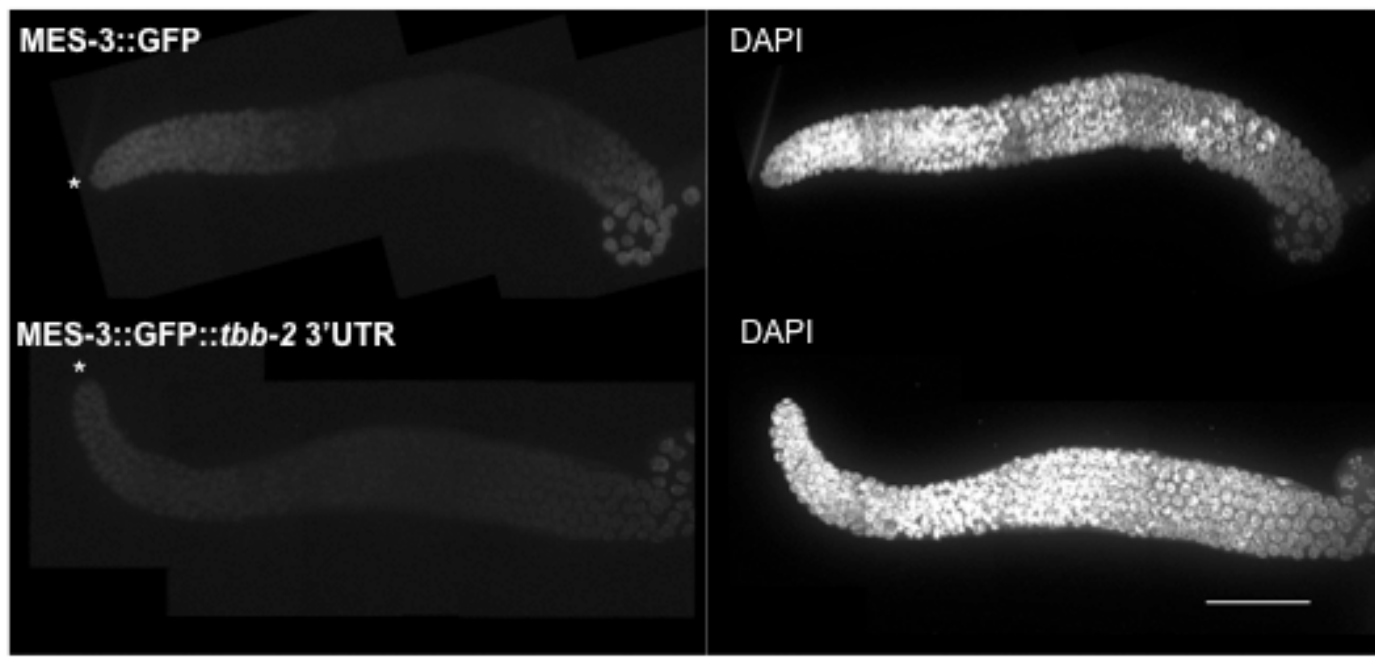

Figure 3.6. MES-3 levels are reduced after exchanging its 3' UTR by tbb-2 3'UTR. Extruded and DAPI-stained germlines. mes-3 3' UTR was exchanged by ubiquitously expressed tbb-2 3' UTR. Analysis of MES-3::GFP expression revealed a decrease in GFP expression compared to control mes-3::GFP. Asterisks $\left(^{*}\right)$ indicate the distal tip of germline Scale bar: $50 \mu \mathrm{m}$.

In any case, upon internal adjusting the intensity levels, we were able to compare the levels of fluorescence between the distal region and the early pachytene region in the distinct allele combinations (Fig 3.7). We have found that exchanging mes-3 3'UTR with tbb-2 3'UTR resulted in the invasion of early pachytene by the GFP signal, in agreement with the observations made by (Xu et al., 2001). The ratio between the levels observed in mitotic region versus early pachytene were lower than the ratio observed when the mes-3(AAA)::GFP allele was analyzed (Fig. 3.7). When both changes (3'UTR and mutation of KEN box) were combined in a single allele, the drop of the GFP signal in the early pachytene region was absent (Fig. 3.7), supporting the idea that both elements were collaborating to maintain MES-3 out from early pachytene region. 
A

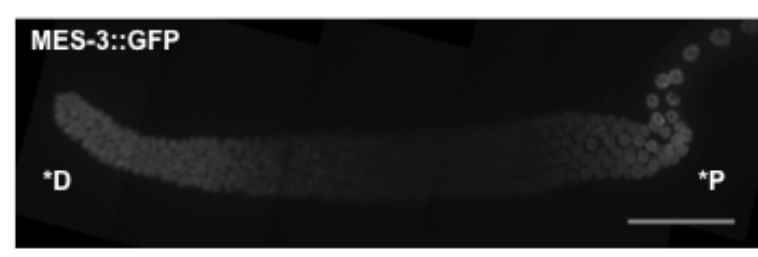

MES-3(AAA)::GFP

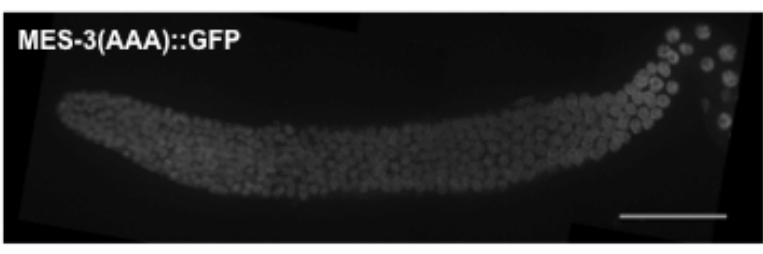

\section{MES-3::GFP::tbb-2 3'UTR}

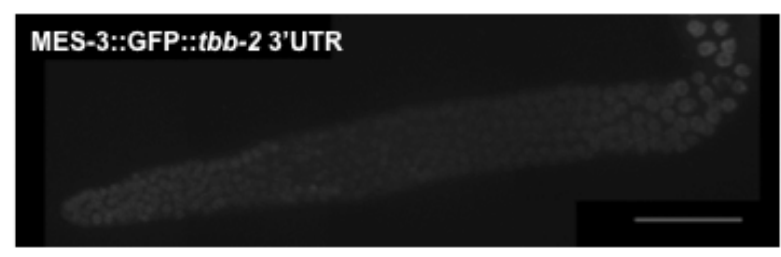

MES-3(AAA)::GFP::tbb-2 3'UTR
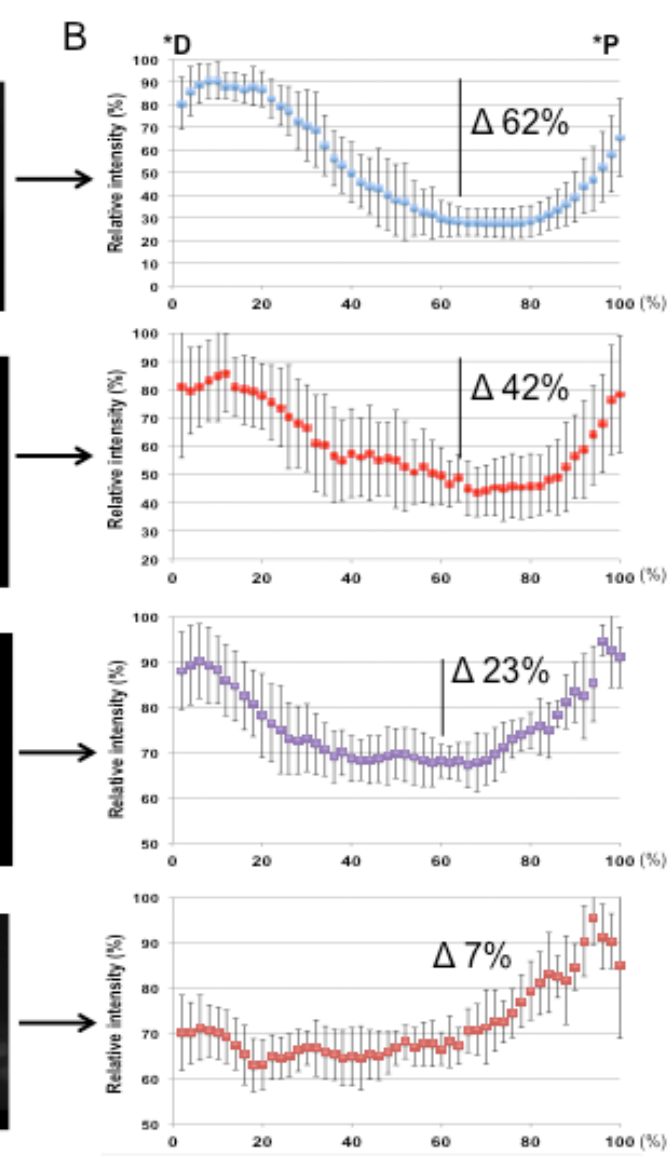

Figure 3.1.7. MES-3 protein levels decay in pachytene through the combined action of GLD-1 and APC $/ C^{\text {FZR-1 }}$. A. MES-3 pattern of expression in germlines of (from top to bottom) : mes-3::GFP, mes3(AAA)::GFP, mes-3::GFP::tbb-2 3'utr and mes-3(AAA)::GFP::tbb-2 3'utr. Degradation of MES-3 through $A P C / C^{F Z R-1}$ was avoided by mutating the KEN-box recognition motif of MES-3 into triple alanines. Translational repression of mes-3 by master regulator GLD-1 was relieved after interchanging mes-3 3'utr

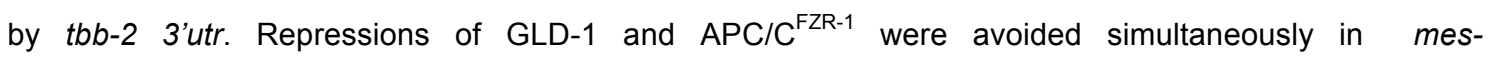
$3(A A A):: G F P:: t b b-2$ 3'utr. Distal end $\left({ }^{*} \mathrm{D}\right)$ and proximal end $\left({ }^{*} \mathrm{P}\right)$. Scale bar: $50 \mu \mathrm{m}$. B. MES-3 expression was quantified along germlines of mes-3::GFP $(n=13)$, mes-3 ${ }^{(A A A)}:: G F P(n=10)$, mes-3::GFP::tbb-2 3'utr $(n=9)$ and mes-3(AAA)::GFP::tbb-2 3'utr $(n=7)$. Plots represent normalized intensity values of GFP versus normalized germline length. Both variables were converted into percentage (\%). GFP was quantified from distal end to diplotene region. For every point, error bars represent the standard deviation. The decay of GFP intensity levels in pachytene region $(\Delta)$ was calculated by subtracting the maximum intensity value $(\%)$ in distal region to the minimum value (\%) in pachytene region.

\subsection{The absence of degradation of MES-3 by APC/C $\mathrm{C}^{\mathrm{FZR}-1}$ dramatically affects fertility}

We have analyzed the brood size of worms carrying the mes-3(AAA) allele. We have observed that mes-3(AAA) worms showed around $30 \%$ of the brood size observed in N2 worms. However, worms carrying the same allele 
tagged with GFP (mes-3(AAA)::GFP) showed no decrease in fertility compared to N2 worms (Fig. 3.8).

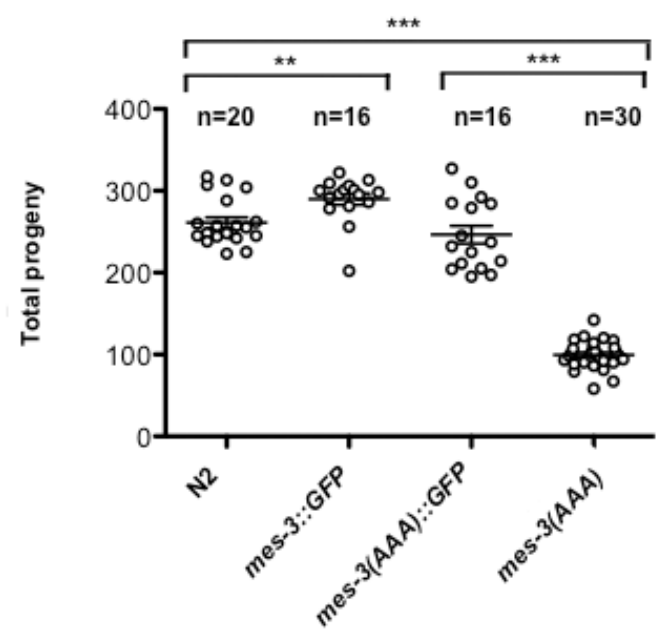

Figure 3.8. KEN-less mes-3 allele, mes-3(AAA), yields fertility defects. mes-3(AAA) suffers a $30 \%$ decrease in fertility levels compared to N2. However, mes-3(AAA)::GFP has a wild-type fertility levels, indicating that GFP masks fertility defects associated with KEN mutation. Fertility assay were carried out at $20^{\circ} \mathrm{C}^{* *}=\mathrm{p}$ value $<0.01 .{ }^{* * *}=\mathrm{p}$ value $<0.001$

In principle, this dramatic difference between the GFP-tagged and the untagged mutant alleles could be attributable to some negative effect of the GFP moiety to the activity of MES-3. However, it is worth remembering that the presence of GFP in an otherwise wild-type mes-3 allele seems to have no apparent effect in fertility, which suggest that the presence of GFP do not affect the proposed activity of MES-3 in germline. We played with the idea that the absence of degradation of MES-3(AAA) by APC/C $C^{\text {FZR-1 }}$ located this protein (either complexed or not with the rest of PRC2 components) in places where in wild-type conditions should not be, and that this mis-localization promoted unscheduled interactions with some cell components that resulted in the impaired fertility. It could be possible that these unscheduled interactions are sensitive to the presence of the GFP moiety, explaining the absence of detrimental effects when analyzed the GFP-tagged mes-3(AAA) allele.

We have combined the mes-3(AAA) allele with mes-4(AAA) alleles (either carrying or not the GFP fusion) (Fig. 3.9). Strikingly, we have observed that the presence of the non-degradable version of MES-4 (mes-4(AAA)) alleviates the fertility drop observed in mes-3(AAA) worms. 
From these results we have extracted two conclusions. The first one concerned the suppression of the fertility defects of MES-3(AAA) in mes$3(A A A)$, mes-4(AAA) double mutants. This suppression could be attributed to the previously described antagonism between MES-4 and PRC2 complex in germline (Gaydos et al., 2012). By a not yet identified molecular mechanism, MES-4 restricts the presence of PRC2 in autosomes, focusing its repressive activity on the $\mathrm{X}$ chromosome. It may well be that in worms carrying the mes$3(A A A)$ allele, the absence of degradation by $A P C / C^{F Z R-1}$ resulted in the unscheduled invasion of genomic regions that somehow affects the fertility of the worms. However, in worms carrying mes-3(AAA) and mes-4(AAA) alleles, the concurrent presence of MES-4(AAA) restricted the invasion of these regions by MES-3(AAA).

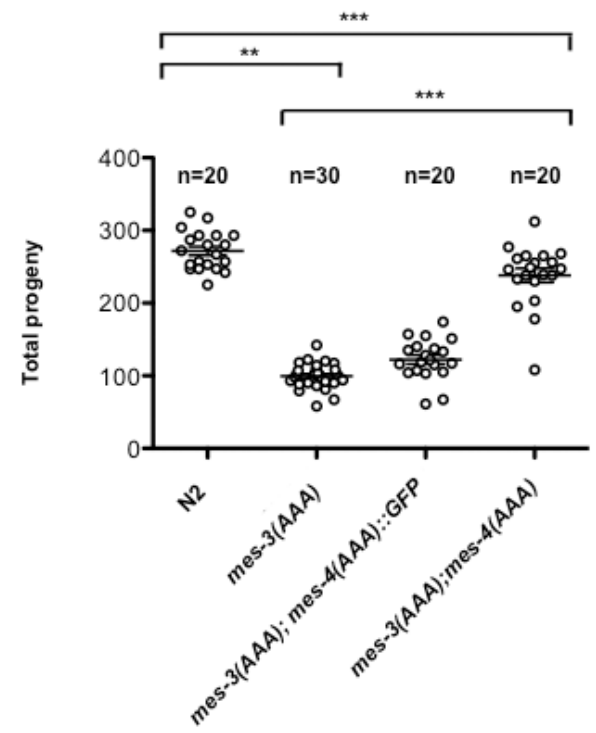

Figure 3.9. mes-4(AAA) alleviates fertility defects associated with mes-3(AAA). The decrease in fertility levels in mes-3(AAA) are suppressed by mes-4(AAA) but not by mes-4(AAA)::GFP. Fertility assay were carried out at $20^{\circ} \mathrm{C} .{ }^{* *}=p$ value $<0.01 .{ }^{* * *}=p$ value $<0.0001$.

The second conclusion concerns to the effects of GFP tagging in the activity of MES-3(AAA) and MES-4(AAA). In both cases, the presence of GFP moiety seems to inactivate the observed effect in fertility of mes-3(AAA) (detrimental) and mes-4(AAA) (protective) worms. However, as we noted above, the presence of the GFP tag seemed not to affect the activity of the wildtype alleles in both cases, at least with respect to fertility and the distribution of the respective protein through the germline. One possible explanation is that in 
both cases the presence of GFP hinders the interaction with some common target, which could be the responsible for the observed defects in fertility.

\subsection{The gonads of mes-3(AAA) worms yield defective eggs}

We were curious about the reasons for the drop in fertility observed in mes-3(AAA) worms. The possibility that this drop was caused by a limited production of sperm (due to a defective spermatogenesis) was discarded because the defect persisted even when exogenous sperm is provided from wild-type males (Fig. 3.10).

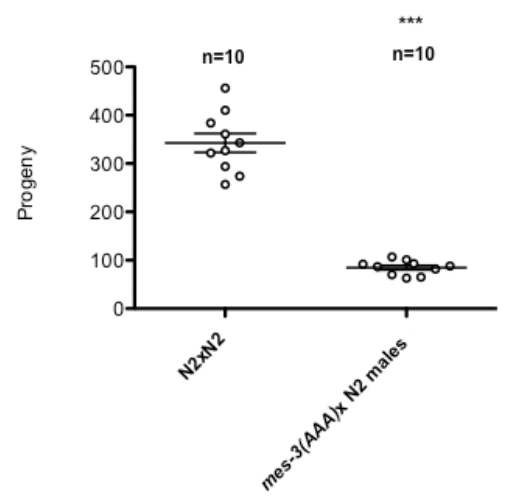

Figure 3.10. Exogenous sperm does not increase fertility levels of mes-3(AAA). Each point represents the total progeny yielded by a single mes-3(AAA) hermaphrodite mated with ten N2 males. For control experiment, N2 hermaphrodites were crossed with N2 males. Progeny of N2 x N2 and mes-3(AAA) $x \mathrm{~N} 2$ were counted until the seventh day, when mes-3(AAA) hermaphrodite ceased to produced offspring. Fertility assay were carried out at $20^{\circ} \mathrm{C} .{ }^{* * *}=p$ value $<0.0001$.

We have also analyzed the gonads from hermaphrodite worms carrying non-degradable versions of mes-3 and mes-4 (without GFP tags), looking for any clue to explain the decrease in fertility observed in mes-3(AAA) worms. In all cases, germlines have a normal appearance. However, in adults carrying the mes-3(AAA) allele, we have observed the presence of defective eggs. Of interest, these defects were lost when the mes-3(AAA) allele was combined with mes-4(AAA) (Fig. 3.11). These observations showed a clear correlation with the fertility data, pointing that the reason for the drop on fertility could be related to the accumulation of these apparent aberrant eggs. 
A

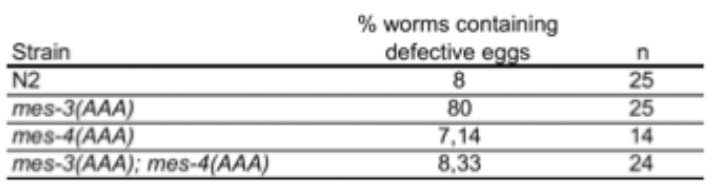

B

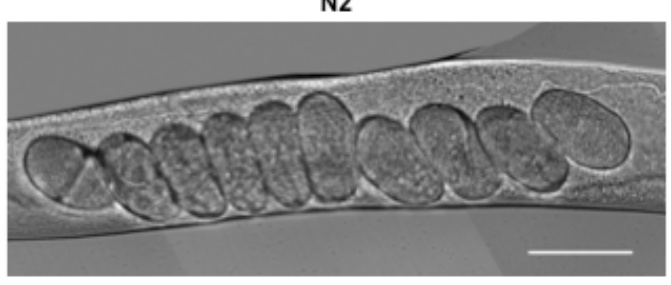

C

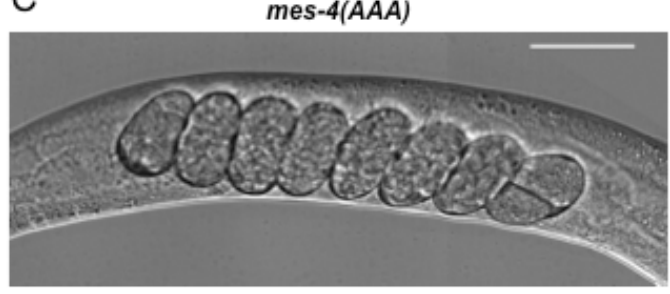

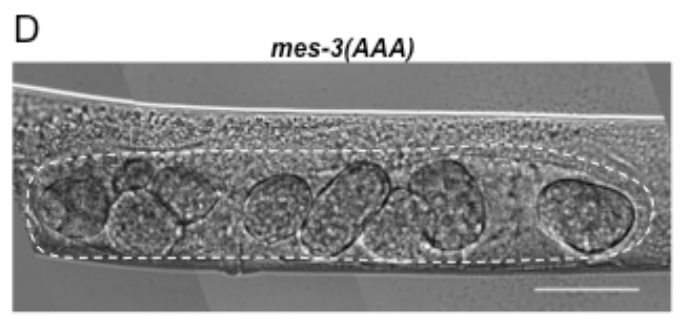

$\mathrm{E}$

mes-3(AAA)

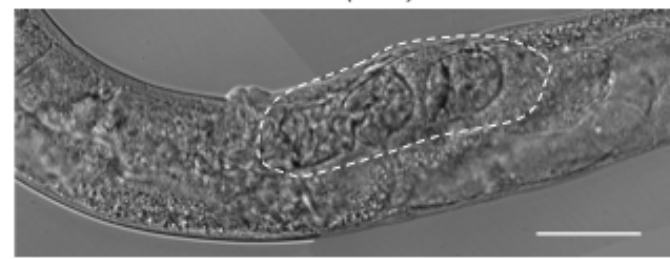

$\mathrm{F}$

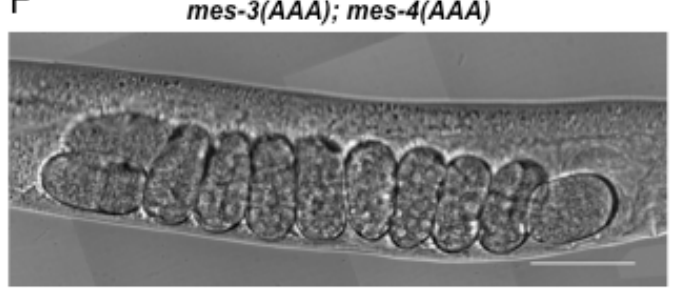

Figure 3.11. mes-3(AAA) mutants produce aberrant eggs. The presence of mes-4(AAA) decreases to wild-type levels the frequency of these defective eggs. A. Table summarizing the percentage of worms containing defective eggs among different strains. (B, C and F). Representative images showing normal eggs within the uterus. (D and E). Representative images of mes-3(AAA) worms containing aberrant eggs. Scale bar: $50 \mu \mathrm{m}$.

\subsection{The invasion of pachytene region could be responsible of fertility defects of mes-3(AAA)}

In previous sections, we have proposed that the observed defects in fertility of mes-3(AAA) worms could be explained by the unscheduled invasion of genomic regions when MES-3 was not degraded by $A P C / C^{F Z R-1}$. We also proposed that the concurrent presence of MES-3(AAA) and MES-4(AAA) somehow could mitigate this occupation. The only gonadal region which occupation by MES-4 and MES-3 is dramatically affected by the activity of $A P C / C^{F Z R-1}$ seems to be the early pachytene. This region of the gonad is particularly appealing to explain the above-mentioned defects in eggs. In the germline, maternal messengers are transcribed from leptotene through pachytene and they are kept inactive through pachytene by the action of GLD1 , which binds and represses translation of these mRNA transcripts (Lee and Schedl, 2001; Wright et al., 2011). Many of these multiple maternal mRNA 
transcripts essential for oocyte and zygote development, and defects in its transcription resulted in severe sterility (Chi \& Reinke, 2006). It could be well that the presence of MES-3(AAA) in pachytene (without the counterbalanced role of MES-4) resulted in some sort of stochastic repression, which in some cases could affect genes required for oocyte and zygote development. To add further support to this idea, we took advantage of our previous observation that exchanging the native 3' UTR region of mes-3 with the tbb-2 3'UTR, MES-3 also invades the pachytene region. Therefore, we have analyzed the fertility as well as the presence of defective eggs in worms carrying the tbb-2 3'UTR as well as the KEN mutation. To our deception, the invasion of pachytene by the presence of alternative UTR does not resulted in a decrease in fertility (Fig. 3.12). However, the fact that the presence of this alternative UTR seemed to alleviate the defects associated to the mes-3(AAA) allele, and the observation that the levels of protein were lower than those observed in a wild-type allele (Fig. 3.6), open the possibility that the absence of effect could be related to low levels of protein.

\subsection{LIN-35 and LIN-15B seems to interact distinctly with the non-degradable mes alleles}

Genetic analyses have shown that the role of MES proteins on the regulation of germline genes is antagonized by a group of transcriptional regulators, among which highlights the transcriptional regulator DREAM complex (EFL-1, DPL-1, LIN-35, LIN-9, LIN-37, LIN-52, LIN-53, LIN-54) (Coustham et al., 2006, Harrison et al., 2006, Wu et al., 2012) and LIN-15B, a THAP domain DNA binding protein, which have been implicated in the silencing of germline genes in somatic (Lee et al., 2017). These genetic studies suggest that competition between the MES chromatin modifiers and the DRM and LIN$15 B$ ensures the proper expression of genes in soma and germline.

It is possible that MES-3(AAA) somehow outcompetes the basal levels of DRM and/or LIN-15B, and consequently the expression of some genes required for fertility were affected. One manner to address this possible explanation was to test whether it was possible to suppress the fertility defects of mes-3(AAA) by increasing the activity of DREAM or LIN-15B. Unfortunately, as far as we 
known, there are no gain-of-function alleles of these complexes. Then, we opted for an opposite manner to address possible genetic interactions between these complexes and our mutant alleles. We have combined loss-of-function alleles in DREAM (using lin-35) and lin-15B with mes-3(AAA) and mes-4(AAA) Interestingly, we have found two different behaviors in these combinations. In the case of lin-35, we have observed a severe drop in fertility levels of the double mutant mes-3(AAA); lin-35 that was alleviated to lin-35 levels when the mes-4(AAA) allele was present (Fig. 3.13A).

A
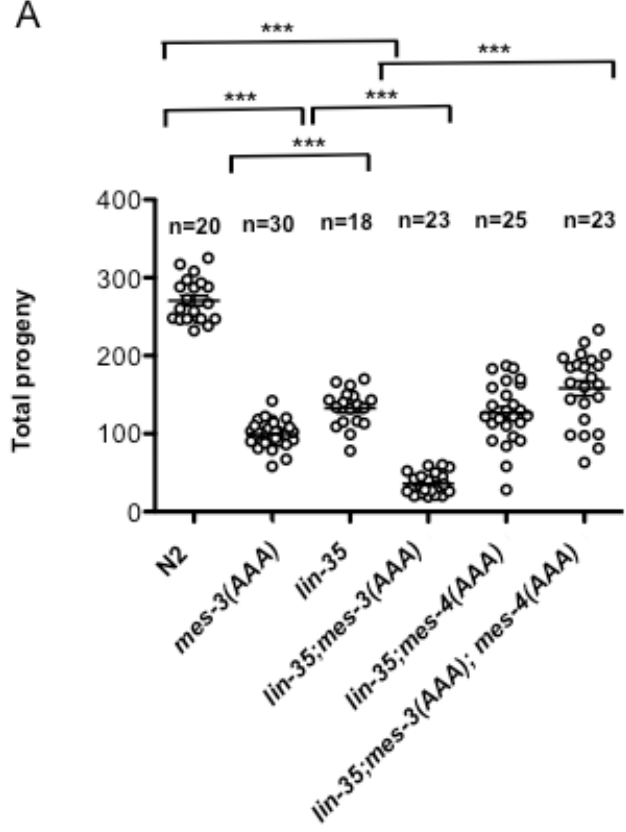

B

\begin{tabular}{lcc}
\multicolumn{1}{c}{ Strain } & $\begin{array}{c}\text { \% worms containing } \\
\text { defective eggs }\end{array}$ & $\mathrm{n}$ \\
\hline $\mathrm{N} 2$ & 8 & 25 \\
\hline mes-3(AAA) & 80 & 25 \\
\hline lin-35 & 9,52 & 21 \\
\hline $\operatorname{lin}-35 ;$ mes-3(AAA) & 80 & 25 \\
\hline lin-35; mes-4(AAA) & 23,08 & 13 \\
\hline lin-35; mes-3(AAA); mes-4(AAA) & 31,82 & 22 \\
\hline
\end{tabular}

Figure 3.13. mes-3(AAA) and lin-35 contribute to fertility defects probably through independent mechanisms. A. mes-3(AAA) and lin-35 single mutants suffer fertility defects. Fertility levels of mes$3(A A A)$; lin-35 double mutants are lower than the respective single mutants. mes-4(AAA) allele dos not affect fertility levels of lin-35. However, in lin-35; mes-3(AAA); mes-4(AAA) triple mutants, fertility levels increase to those of lin-35. Fertility assay were carried out at $20^{\circ} \mathrm{C}$. ${ }^{* * *}=p$ value $<0.0001$. B. Table summarizing the percentage of worms containing defective eggs among different strains. Results from N2 and mes-3(AAA) were included from figure 2.1.11 A for comparative purposes. lin-35 mutant yields a similar percentage of defective eggs than N2, indicating that sterility defects of mes-3(AAA) and lin-35 stem from different origins.

Analysis of the frequency of defective eggs shows that the percentage of aberrant eggs does not increase in mes-3(AAA); lin-35 double mutants compared to mes-3(AAA) single mutants. This indicates that the synthetic 
interaction regarding fertility between mes-3(AAA) and lin-35 does not correlate with the appearance of defective eggs (Fig. 3.13B).

In the case of lin-15B, however, there is an additive effect with the distinct mes-3(AAA) and mes-4(AAA) alleles (Fig. 3.14). It is remarkable that fertility defects and the frequency of aberrant eggs, as well, in mes-3(AAA) are partially alleviated in mes-3(AAA); lin-15B double mutants.

A
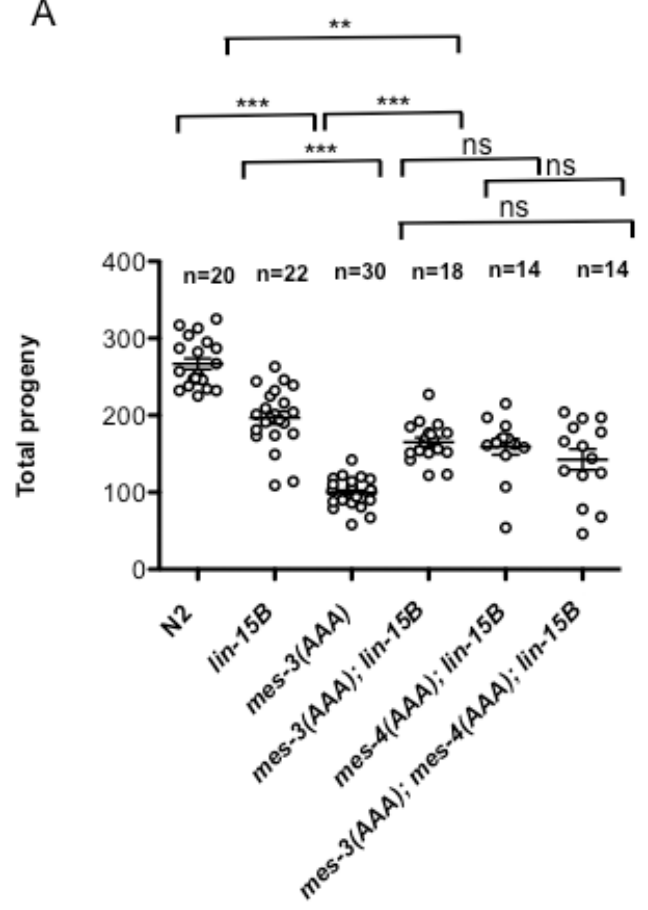

B

\begin{tabular}{lcc}
\multicolumn{1}{c}{ Strain } & $\begin{array}{c}\text { \% worms containing } \\
\text { defective eggs }\end{array}$ & $n$ \\
\hline N2 & 8 & 25 \\
\hline mes-3(AAA) & 80 & 25 \\
\hline lin-15B & 11,11 & 27 \\
\hline lin-15B; mes-3(AAA) & 13,33 & 15 \\
\hline lin-15B; mes-4(AAA) & 26,67 & 15 \\
\hline lin-15B; mes-3(AAA); mes-4(AAA) & 23,53 & 17 \\
\hline
\end{tabular}

Figure 3.14. lin-15B alleviates fertility defects of mes-3(AAA). A. lin-15B mutant in combination with mes-3(AAA), mes-4(AAA) or mes-3(AAA); mes-4(AAA) double mutants, reduces its fertility levels. It is noteworthy that lin-15B partially alleviates mes-3(AAA) fertility defects. Fertility assay were carried out at $20^{\circ} \mathrm{C}$. ${ }^{* *}=\mathrm{p}$ value $<0.01 .{ }^{* *}=\mathrm{p}$ value $<0.000$. ns: no significant $1 \mathrm{~B}$. Table summarizing the percentage of worms containing defective eggs among different strains. Results from $\mathrm{N} 2$ and mes-3(AAA) were included from figure 2.1.11 A for comparative purposes. In agreement with fertility results, lin-15B diminishes in mes-3(AAA) the percentage of worms carrying defective eggs.

\section{7. fzr-1(sal19) allele is a complete deletion of the fzr-1 coding sequence}

To unveil the roles played by $\mathrm{APC} / \mathrm{C}^{\mathrm{FZR}-1}$ in $C$. elegans development, we sought to construct an allele carrying a full deletion of the fzr-1 ORF using CRISPR technology. For that, we have combined two sgRNA, one designed to direct the cut of Cas9 complex at the start codon, and the second one directed 
against the stop codon of $f z r-1$ ORF. The concurrent action of Cas 9 complexes carrying the two sgRNAs removed the entire ORF, leaving a double-strand break (DSB) that, upon repair using the NHEJ pathway, resulted in a deletion of the entire ORF. We microinjected in N2 worms a mix consisting of Cas9 complexes loaded with the sgRNAs designed against fzr-1 ORF. We have included in the injected mix the co-injection marker based on dpy-10(cn64). From plates showing a high frequency of roller and dumpy worms, we analyzed single worms using primers flanking the expected fzr-1 deletion (Fig. 3.15). We have isolated two independent alleles, which were sequenced. Finally, we chose the one that showed the expected borders, fzr-1(sal19). After backcrossing several times worms carrying this allele, we have found that it was not possible to obtain fertile fzr-1(sal19) homozygous worms, suggesting an essential role of FZR-1. To facilitate the handle of these worms, we kept the fzr1 (sal19) allele in heterozygosis with the aid of $m / n 1$, a balancer for chromosome II.

A

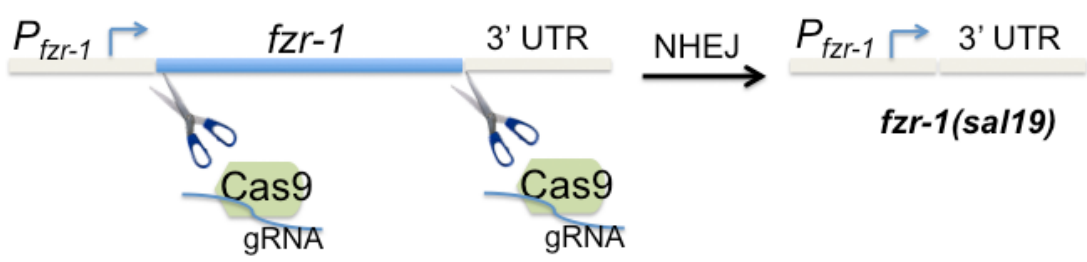

B

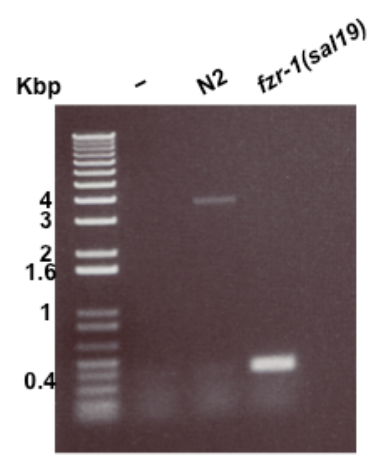

Figure 3.15. fzr-1 ORF was deleted by CRISPR-Cas9. A. Two Cas9-gRNA complexes directed against start and stop codon released $f z r-1$ ORF. The DSB generated was repair by NHEJ, generating a null allele of $f z r-1, f z r .1(s a l 19)$. B. Deletion of $f z r-1$ (sal19) was confirmed by PCR with flanking oligos check fzr-1 1 and check fzr-1 2. 


\section{8. fzr-1(sal19) develop into sterile hermaphrodites}

We analyzed whether heterozygous $\mathrm{fzr}-1(\mathrm{sal} / 9) / \mathrm{m} \ln 1$ produce viable homozygous $f z r-1(s a l 19)$ among their progeny. We have observed that fzr$1(\mathrm{sal19}) / \mathrm{m} \ln 1$ produce progeny of the three possible genotypes: fzr1(sal19)/mIn1, mIn1/mIn1 and fzr-1(sal19)/ fzr-1(sal19) almost following the expected mendelian inheritance (50\% fzr-1(sal19)/mIn1, 25\% $\mathrm{mln} 1$ and $25 \%$ fzr-1(sal19)) (Fig 3.16). Homozygous fzr-1(sal19) hermaphrodites reach adulthood but present several defects. We found that they were unc, sometimes showed super coil movements, and developed a protruding vulva. However, the most obvious phenotype was that they were sterile: $f z r-1(s a l 19)$ adults do not possess a line of eggs within the uterus, never lay eggs, and showed no progeny at all (Fig 3.17).

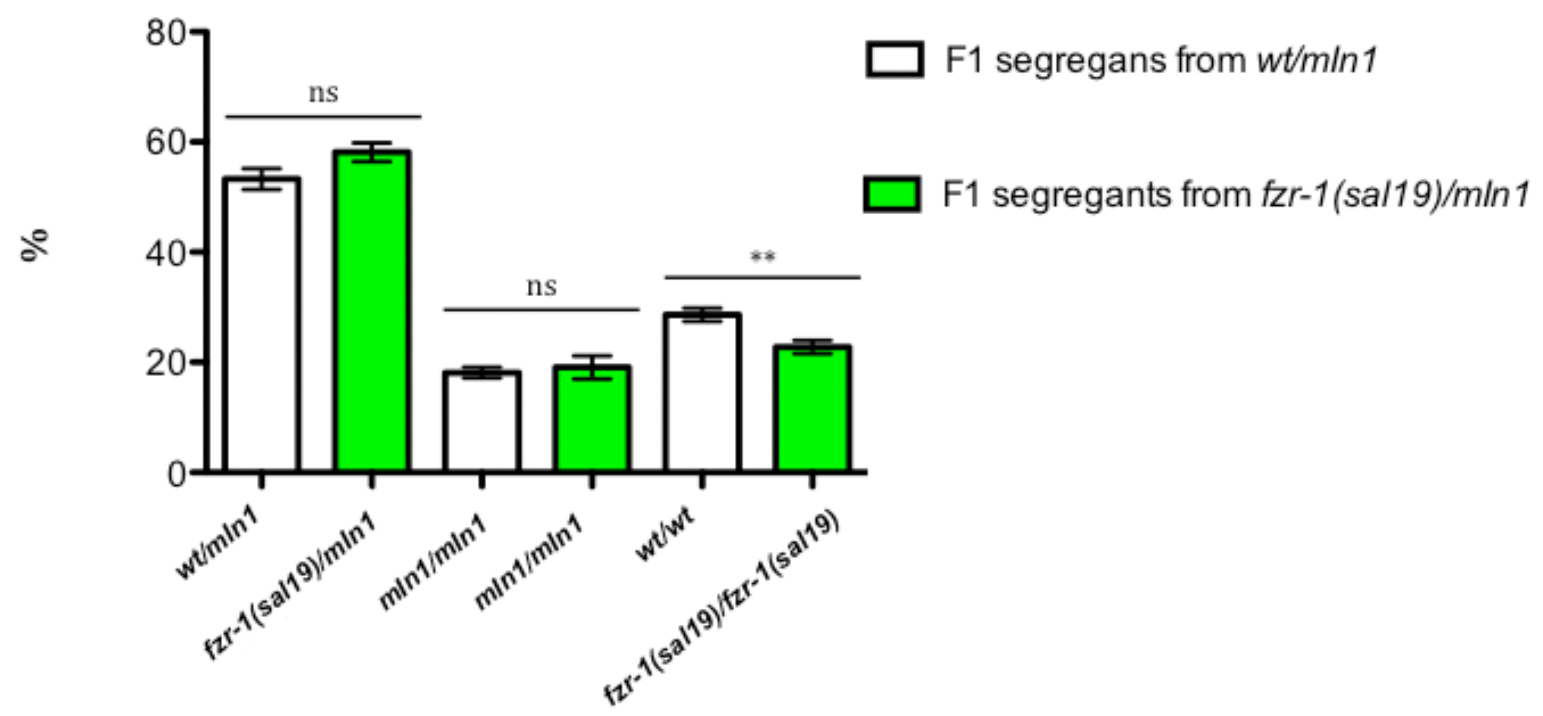

Figure 3.16. fzr-1(sal19)/mIn1 yields viable homozigous fzr-1(sal19)/fzr-1(sal19) following a mendelian inheritance. Frequency (\%) of genotypes segregated among F1 progeny by Po control $w t / m I n 1$ (white columns) versus Po $f z r-1(s a l 19) / m I n 1$ (green columns). One wt/mln1 (Po) or fzr1(sal19)/mIn1 (Po) was added per dish. A total of, approximately, $100 \mathrm{~F} 1 \mathrm{~s}$ ( $\geq$ L3 stage) were counted per dish $(n=10)$ and classified according to their genotype: homozigous $m / n 1 / m / n 1$ posses a green pharynx and are dumpy, heterozygous $w t / m / n 1$ or $f z r-1(s a l 19) / m I n 1$ have a green pharynx and homozigous $w t / w t$ or fzr-1(sal19)/ fzr-1(sal19) do not possess a green pharynx nor are dumpy. Error bars indicate the standard error of mean (SEM). ns: no significant. ${ }^{* *}=p$ value $<0.01$. 
To directly assign the observed defects from worms carrying fzr-1(sal19) to a loss-of-function of this allele, we have tried to complement these defects with an ectopic wild-type fzr-1 allele. For that, we have constructed an ectopic copy of $f z r-1$, including its native promoter and its $3^{\prime}$ UTR to be inserted at Universal $\mathrm{MosCl}$ for chromosome $\mathrm{V}$. The fzr-1(sal19) worms carrying the inserted ectopic copy of $f z r-1$ were indistinguishable from wild-type worms concerning fertility levels (Fig 3.17) and the other observed defects (Unc, $\mathrm{Pvl} / \mathrm{Evl}$ ), confirming that the deficiencies were exclusively attributed to the absence of $f z r-1$ function.

A

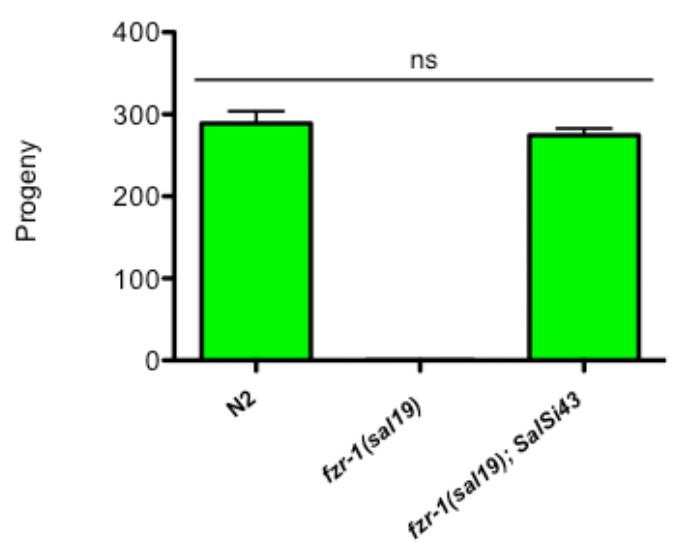

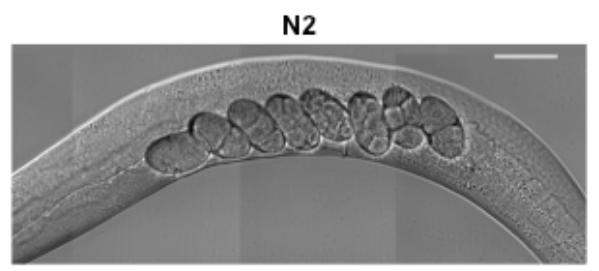

fzr-1(sal19)

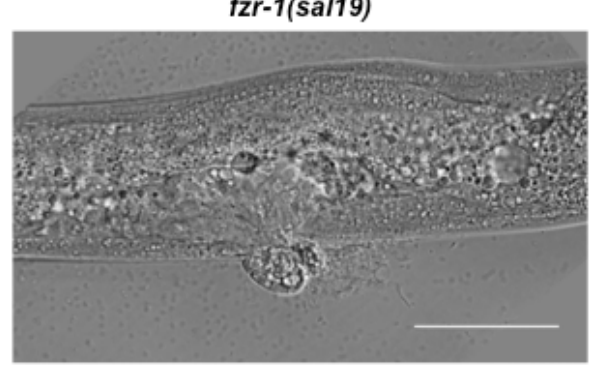

fzr-1(sal19); salSi43

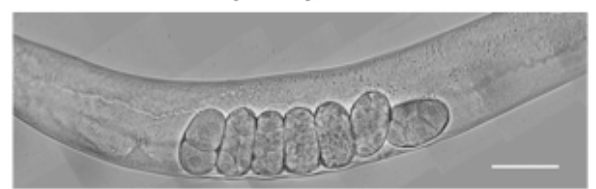

Figure 3.17. fzr-1(sal19) mutants are sterile. A. Fertility assay for $N 2(n=9), f z r-1(s a l 19)(n=40)$ and $f z r-$ 1(sal19); SalSi43 (n=8). fzr-1(sal19) mutants are sterile. Complementation with rescuing transgene SaliS43, which expresses $P_{f z r-1}:: f z r-1:: 3$ 'UTR fzr-1, restores fertility of $f z r-1$ (sal19). Error bars indicate the standard error of mean (SEM). ns, no significant. B. Representative DIC images of mid bodies of gravid N2 (upper image), fzr-1(sal19) with protruding vulva and no eggs (middle image) and fzr-1(sal19); salSi43, which is indistinguishable from N2 (lower image). Scale bar: $50 \mu \mathrm{m}$.

We have also isolated $f z r-1$ (sal19) males. In addition to the sterility phenotype, we have observed that the male tail showed an abnormal morphology missing rays (Fig 3.18). 
A

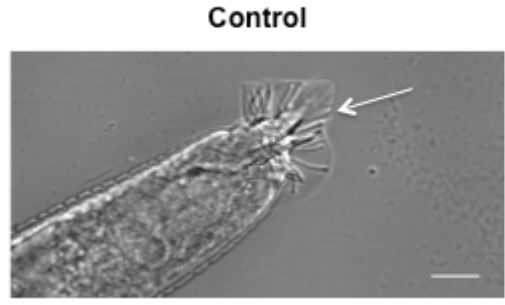

B

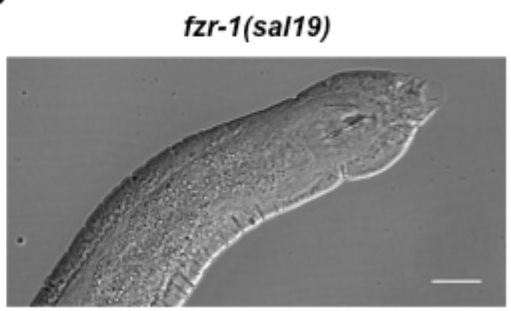

Figure 3.18. Somatic gonadal cells of fzr-1(sal19) males are properly specified. A. Control male tail features rays (arrow). B. fzr-1(sa/19) male tail miss rays. him-5 allele was utilized to get males easily. Scale bar: $20 \mu \mathrm{m}$.

\section{9. fzr-1(sal19) hermaphrodites failed to form gonadal arms.}

To address the reasons for fertility defects in $f z r-1$ (sal19), we have analyzed the gonad morphology in these mutant worms (Fig 3.19A).

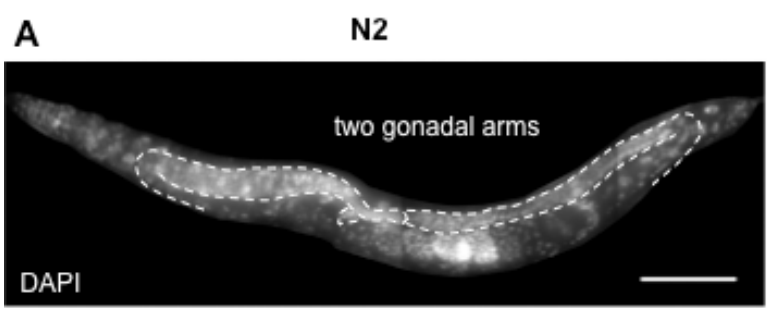

C

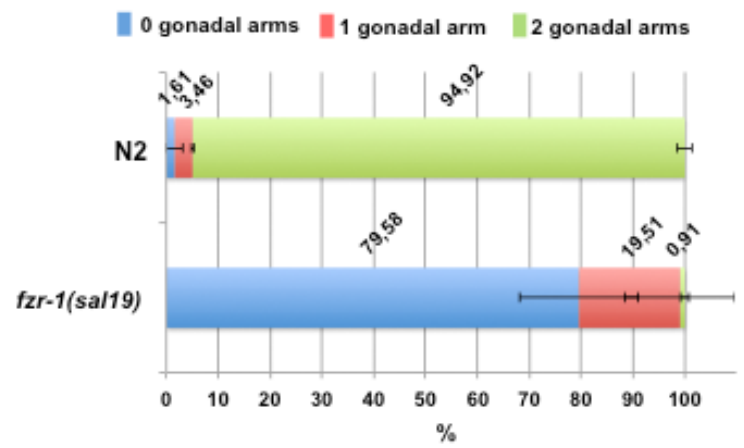

B fzr-1(sal19)
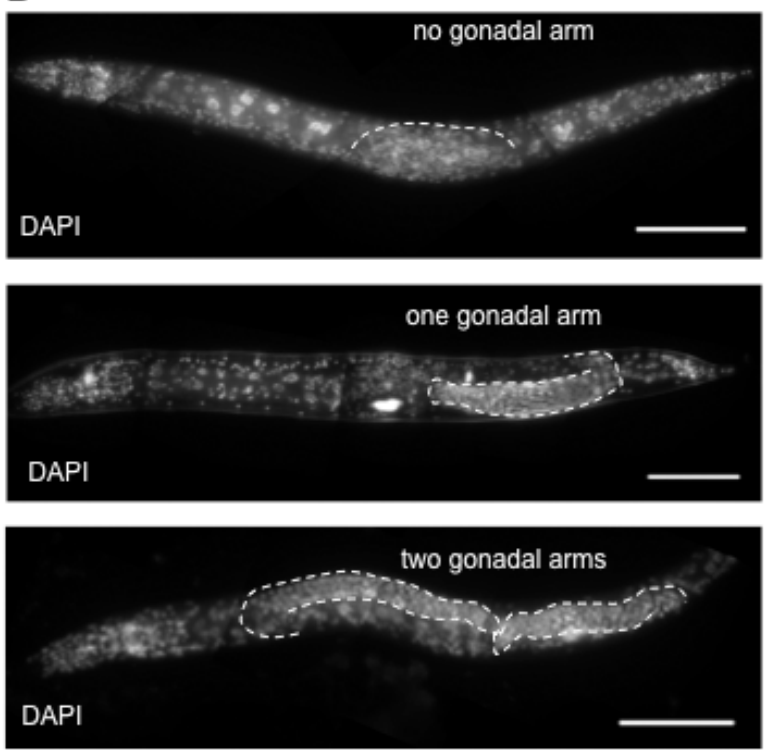

Figure 3.19. fzr-1(sal19) hermaphrodites fail to extend gonadal arms. (A \& B). DAPI-stained young adult hermaphrodites. Gonads are outlined. A. Control N2 with two gonadal arms. B. fzr-1(sal19) mutants usually lack gonadal arms (upper image). In few occasions, fzr-1(sal19) produces gonads (middle and lower images). C. Frequencies (\%) of worms with none, one or two gonadal arms, for both N2 ( $n=58)$ and $f z r-1(s a / 19)(n=166)$. Bars represent the average of three independent experiments. Error bars indicate SD. Scale bar: $100 \mu \mathrm{m}$. 
Hermaphrodite wild-type gonads were composed of two U-shaped gonads However, most fzr-1(sal19) hermaphrodites lacked both gonadal arms. Only a small percentage $(20 \%)$ of mutants showed one gonadal arm (more frequently the posterior arm), and more scarcely (1\%) two gonadal arms (Fig 3.19B and C).

C. elegans possesses different gonad development programs depending on sex. These different programs are linked to the distinct gonad structure in hermaphrodite and male. Hermaphrodites contain two U-shaped gonads, and males possess one J-shaped gonad (Fig 3.20A). We have also analyzed male fzr-1(sal19) worms for the presence of gonads. In order to get males easily, we combined fzr-1(sal19) with him-5 (him stands for High Incidence of Males), which yields roughly $30 \%$ of male progeny. Strikingly, all fzr-1(sal19) male analyzed carried gonads consisting of a single elongated arm indistinguishable from control gonads (Fig 3.20B and C). To discard him-5 affecting the effects of lack of FZR-1 function in gonad development, we have collected randomly appearing males from $\mathrm{fzr}-1$ (sal19) and analyzed by DAPI staining their gonads. Coherently, we have also observed elongated gonadal arms in these cases.
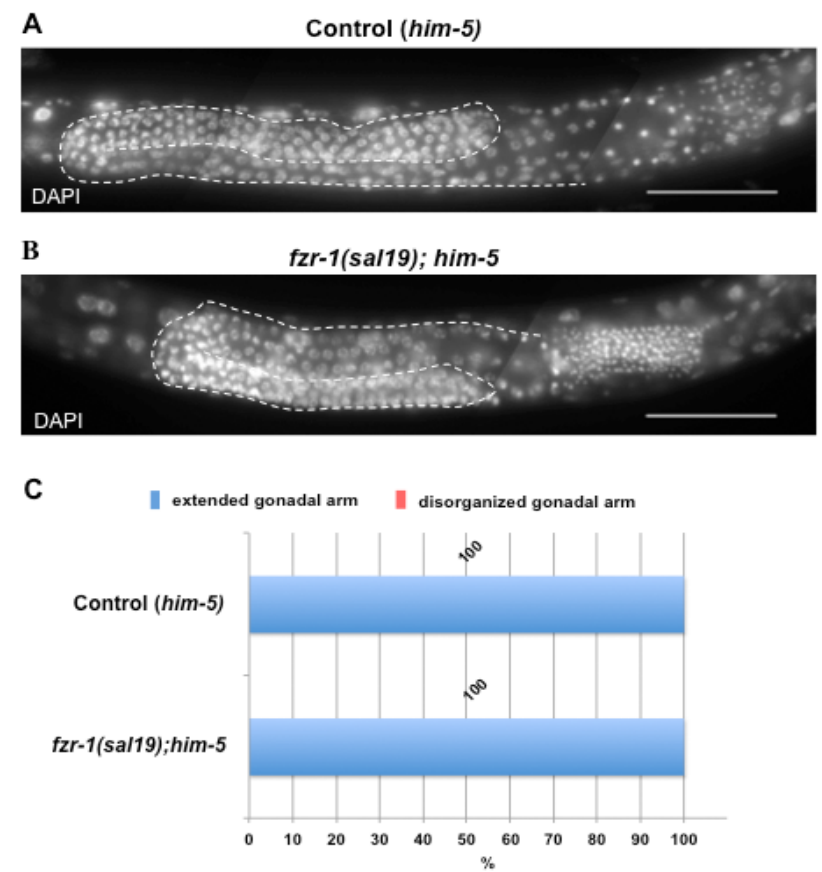

Figure 3.20. fzr-1(sal19) males develop an extended J-shaped gonadal arm. (A-B). DAPI-stained young adult males. Gonads are outlined. A. Control (him-5) male gonad. B. fzr-1(sal19); him-5 always contains a J-shaped gonadal arm. C. Frequencies (\%) of control (him-5) ( $\mathrm{n=60}$ ) and fzr-1(sal19); him-5 males $(n=52)$ with extended or disorganized. Bars represent the average of three independent experiments. Error bars indicate SD. Scale bar: $50 \mu \mathrm{m}$. 
We have tried to address at which stage the gonadal development was impaired in hermaphrodites. Germline developed from two primordial germ cells (PGCs), named Z2 and Z3. We followed germline development from $L 1$ to adult. We have used the germline reporter PGL-1:: GFP. PGL-1 is a component of the P-granules, which was necessary to maintain germ identity by regulating transcripts (reviewed in (Updike \& Strome, 2010)). Figure 3.21 shows the progression of germline development comparing control and fzr-1(sal19) worms. We have found that Z2 and Z3 were present in L1 fzr-1(sal19) worms. Indeed, we have observed no differences at L1 and L2 stages between N2 and fzr-1(sal19) worms with respect germlines. However, once fzr-1(sal19) reached L3, differences emerged. From this point, control gonads elongated, but in fzr1(sal19), the gonads were composed of very few enlarged germline cells that seemed to be arrested. In these worms, no typical gonad structure appears, and the scarce germline was packed in the center of the worm (Fig 3.21E and F).
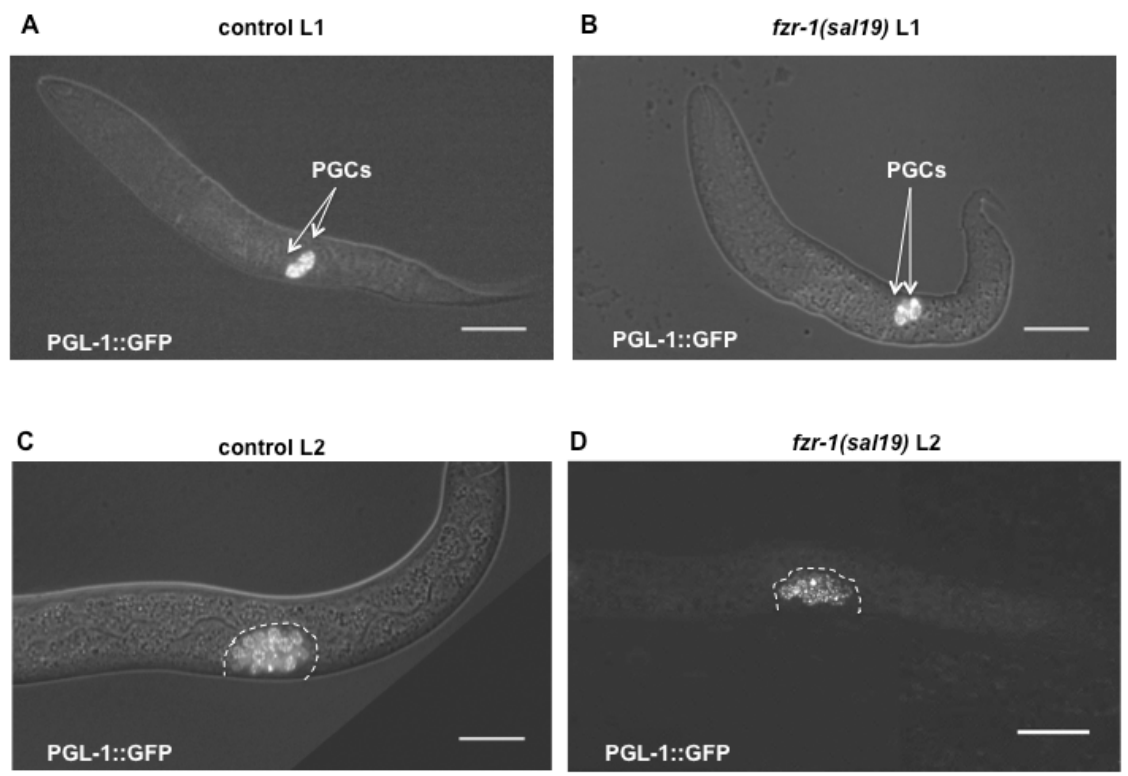

Figure 3.21. fzr-1(sal19) produces Primordial Germ Cells (PGCs) that fail to produce extended germlines. A-F. Germ cells express pgl1::GFP reporter. A \& B. PGCs are indicated by arrows in control and $f z r-1$ (sal19) L1, respectively. C \& D. Germline is outlined in control and $f z r-1$ (sal19) L2, respectively. E \& F. Germline development from L3 to young adults in controls and $f z r-1(s a l 19)$. $n>10$. A-D. Images represent a merge between Nomarsky and PGL-1::GFP. Scale bar: $20 \mu \mathrm{m}$. E \& F. Scale bar: $50 \mu \mathrm{m}$. 

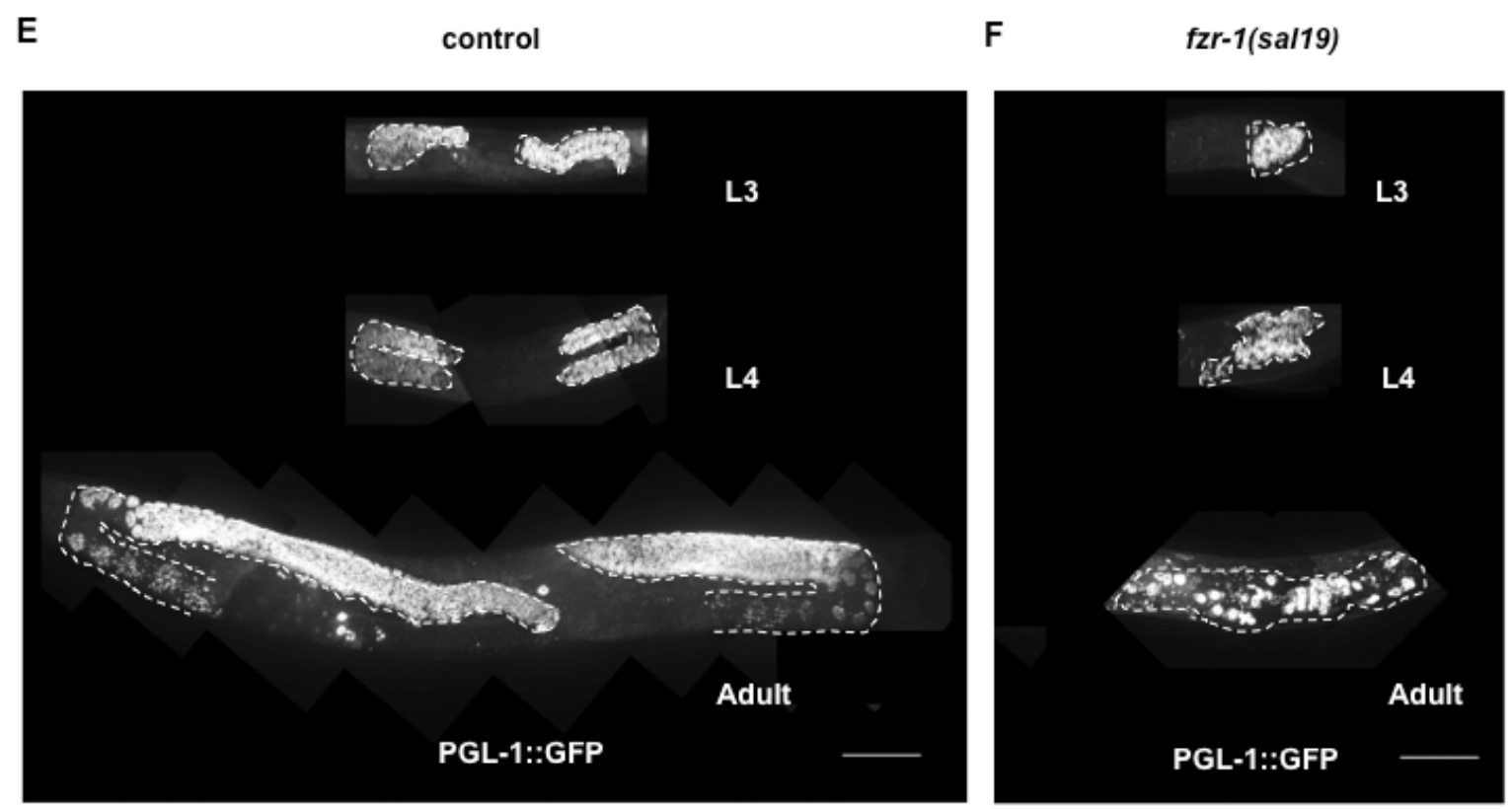

Figure 3.21. Cont

\subsection{Hermaphrodite fzr-1(sal19) worms were defective in distal tip cell formation.}

Germ cell maintenance and germline extension rely on a particular cell, the Distal Tip Cell (DTC), which acts as a stem-cell niche. DTCs maintain germ cell identity by extending processes on germline, secreting Notch ligands that activate the Notch pathway in germ cells (Byrd et al., 2014). Also, DTCs can migrate, leading the outgrowth of gonads. We played with the idea that the observed defects in germ cell division and gonad extension in fzr-1(sal19) could be attributed to defects in DTCs. To analyze DTCs within fzr-1(sal19), we used the Notch Ligand reporter lag-2::GFP (Blelloch et al., 1999). We have found that fzr-1(sal19); lag2p::GFP adults lacked one or both DTCs (Fig. 3.22). We have observed that the frequency of worms lacking gonadal arms (Fig. 3.19.) correlates with the frequency of worms showing the absence of DTC, suggesting that defects in DTC are behind the inability to produce gonad development. We synchronized fzr-1(sal19); lag2p::GFP and let them grow to 1day-old adults. Then, with the help of a dissecting scope, they were split into those with none, one, and two DTCs. Next, they were DAPI stained, revealing that worms without DTC never elongated gonadal arms. Worms with one or two DTCs had one or two gonadal arms, respectively (Fig. 3.23). Finally, we wondered whether $\mathrm{fzr}$-1(sal19) worms with at least one elongated gonadal arm 
could yield progeny. So, we have isolated L4 fzr-1(sal19); lag2p::GFP with one or two DTCs and let them grow until they die. None of them had progeny nor laid eggs.

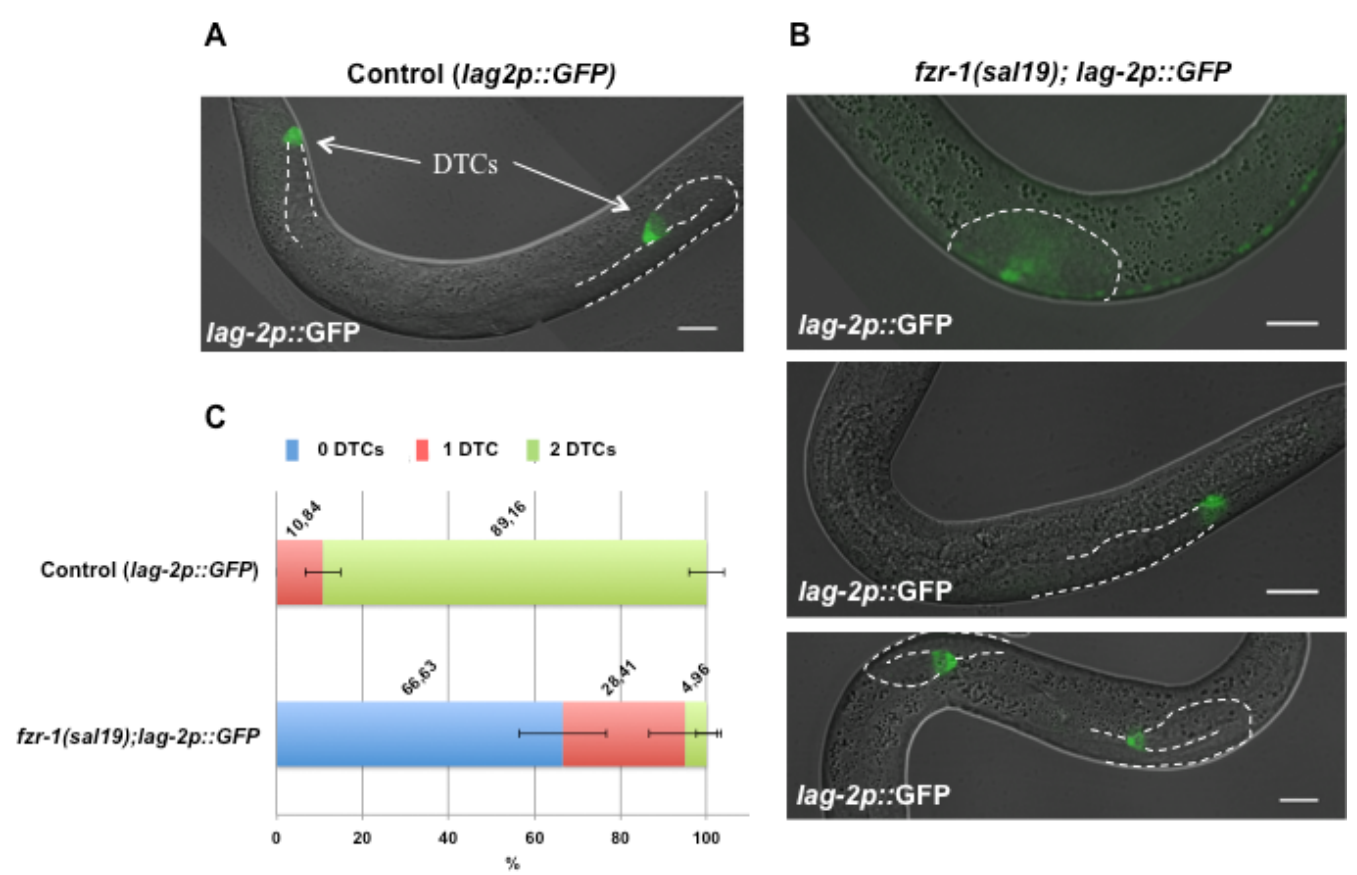

Figure 3.22. fzr-1(sal19) miss DTCs. A. Control strain JK2049 expresses DTC marker lag2-p::GFP. Both DTCs are indicated. B. fzr-1(sal19) misses DTCs (upper and middle images). Rarely, fzr-1(sal19) produces two DTCs (lower image). Images from A and B are a merge between Nomarsky and lag-2p::GFP signal. C. Frequencies (\%) of young adult worms with none, one or two DTCs, for both control and fzr1(sal19). Bars represent the average of three independent experiments. Error bars indicate SD. For every experiment, DTCs from $n>40$ were counted using a dissecting scope. Scale bar: $20 \mu \mathrm{m}$.

Because $f z r-1$ (sal19) males showed an extended gonad, we were curious about whether male DTC (mDTC) was produced. Males also contain two DTCs, which were located together at the single gonad's distal part. Because in mDTCs the lag2p::GFP signal have been reported to be faint, we choose the brighter arg-1p::GFP reporter (Large \& Mathies, 2010) and constructed fzr-1(sal19); him-5; arg-1p::GFP strain. We counted the number of mDTC per worm. In agreement with the presence of single-arm gonads in fzr1(sal19) males, we observed the presence of 2 DTCs per gonad (Fig. 3.24).

In summary, we have found that the absence of FZR-1 resulted in a high frequency of absence of DTC in hermaphrodites, but seemed not to affect the formation of mDTC. In any case, despite the presence of male gonads or occasionally hermaphrodite gonads, none fzr-1(sal19) worm was fertile. 
A

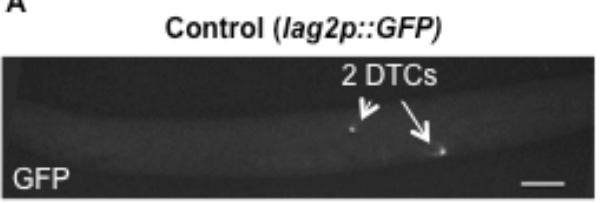

C
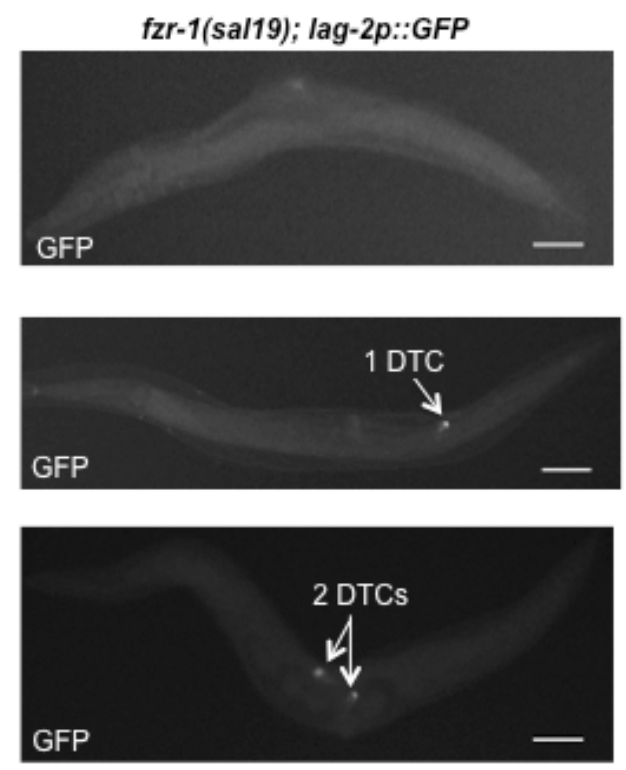

B

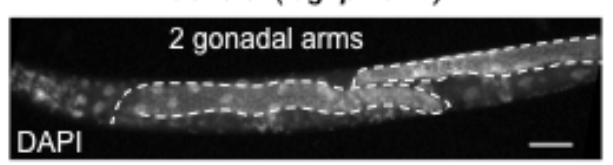

D
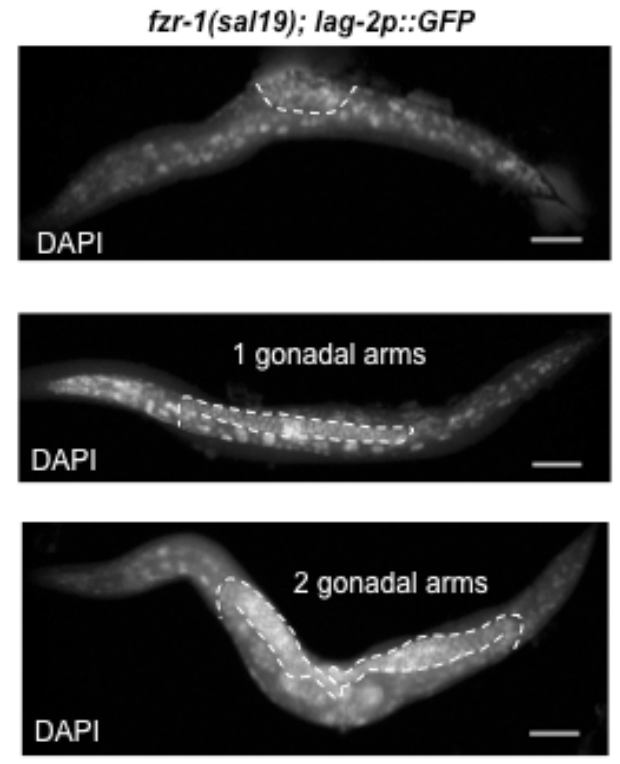

Figure 3.23. DTCs produced in fzr-1(sal19) lead gonadal elongation. A. Control strain JK2049 (lag$2 p:: G F P$ ) produce two DTCs that elongate one gonadal arm each (B). (C and D). $f z r-1$ often miss both DTCs. When produced, DTCs always lead gonadal arm elongation in $f z r-1$ (sal19). Indiviuals $f z r-1$ (sal19) missing both DTCs (C, upper image), never extended gonadal arms (D, upper image). fzr-1(sal19) mutants with one DTC contain (C, middle image) one gonadal arm (D, middle image). fzr-1(sal19) that produce both DTCs (C, lower image), develop two U-shaped gonads (D, lower image). Scale bar: $50 \mu \mathrm{m}$.
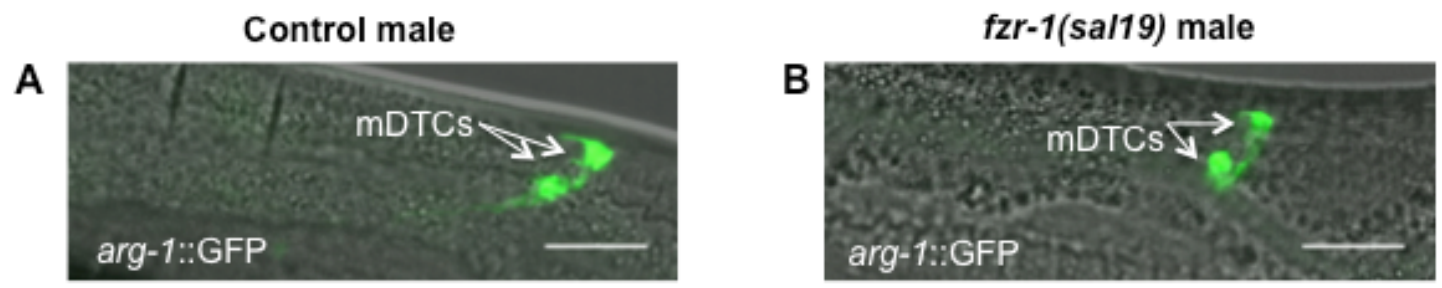

Figure 3.24. fzr-1(sal19) males produce mDTCs. A. Control males produce two mDTCs observed with reporter $\arg -1:: G F P(\mathrm{n}=43)$. B. fzr-1(sal19); arg-1::GFP males produce two mDTCs $(\mathrm{n}=41)$. (A.B). In order to get males easily, him-5 allele was utilized. Scale bar: $20 \mu \mathrm{m}$.

\subsection{Somatic gonad primordium was formed in fzr-1(sal19)}

DTCs are part of a complex organ named somatic gonad. Briefly, this organ plays a significant role in the development of germline. Somatic gonad 
co-develops with germline, enveloping it and leading its outgrowth. Spermatheca and uterus are also components of the somatic gonad. The origin of all cells that compose the somatic gonad could be traced back to two founder cells, the somatic gonad precursors (SGPs) Z1 and Z4. We have analyzed whether a defect in SGPs formation caused the absence of DTCs observed in fzr-1(sal19) worms. As a reporter of SGPs, we have used ehn3p::GFP, which is expressed in Z1 and Z4 (Welchman et al., 2007). Worms were synchronized, and hatched L1 larvae were analyzed. We have observed that both Z1 and Z4 are present in $f z r-1$ (sal19), (Fig. 3.25) indicating that the absence of SGPs does not explain the lack of DTCs. Moreover, Z1 and Z4 in fzr-1(sal19) worms seemed to locate ventrally. It has been reported that these correct position is necessary to ensure the formation of DTCs. Mutants in hnd-1, ehn-3, and SWI/SNF components, which often generate misoriented Z1 or Z4, also resulted in DTCs absence (Large \& Mathies, 2010, Large \& Mathies, 2014, Mathies et al., 2003).
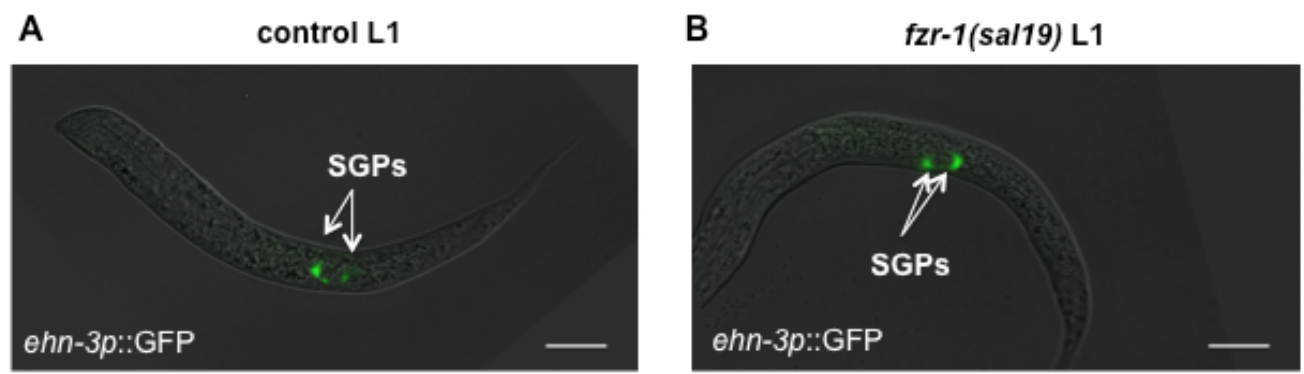

Figure 3.25. fzr-1(sal19) produces SGPs, founder members of somatic gonad. A. Control strain RA45

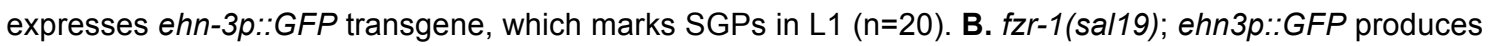
both SGPs $(n=28)$. L1 larvae were collected after synchronization by treatment with bleaching solution. Scale bar: $20 \mu \mathrm{m}$.

\subsection{The absence of DTCs in fzr-1(sal19) cannot be explained by defects in the Wnt pathway.}

Previous results showed that, at early stages, SGPs and PGCs are produced in $f z r-1(s a / 19)$, indicating that a proper gonadal primordium was formed (Z1, Z2, Z3, and Z4). To form the somatic gonad, the SGPs achieved a series of asymmetric divisions following a distal-to-proximal axis (D-P axis). The 
outcome of these asymmetric-cell divisions was determined by the noncanonical Wnt pathway. To produce DTCs, each SGP is submitted to 2 cell division rounds. In the first round, Z1 (Z4) resulted in two cells, Z1a (Z4p) receiving Wnt signal and Z1p (Z4a) without the Wnt signal. The second round of division from Z1a (Z4p) resulted in DTC (receiving Wnt signal) and blast somatic gonad cell (not receiving Wnt signal). In other words, the formation of DTC from SGP required an active Wnt pathway. Since there was a previous report in Drosophila linking the Wnt pathway and $\mathrm{APC} / \mathrm{C}^{\mathrm{Fzr} 1}$, we wondered whether the observed defect in DTC formation was due to a defective Wnt pathway in the absence of FZR-1 function.

The non-canonical Wnt pathway that regulates the somatic gonad formation in $C$. elegans relies on the nuclear ratio of its two central regulators: SYS-1 ( $\beta$-catenin) and POP-1 (TCF). The heterodimer SYS-1/POP-1 acts as a transcriptional activator of genes downstream Wnt, while monomeric POP-1 behaves as a repressor of transcription. Distal cells, where the Wnt pathway is active, showed a high ratio SYS-1/POP-1 in the nucleus. On the contrary, proximal cells, which do not receive Wnt signal, maintain a low ratio SYS1/POP-1. The analysis of nuclear levels of a GFP: POP-1 fusion has been reported as a straightforward manner to determine the activity of the Wnt pathway in Z1/Z4 daughters (Siegfried et al., 2004). We have tried this approach using worm strains carrying this fusion protein. However, in our hands, we were unable to detect GFP::POP-1 fluorescence associated with Z1/Z4 cells even in control worms, although we detected nuclear GFP fluorescence later in development (Fig. 3.26). Because of our inability to address the Wnt activity directly in SGPs, we tried to accumulate pieces of evidence about how the Wnt pathway operated in fzr-1(sal19). Mutations affecting the Wnt pathway in C. elegans resulted in the D-P axis's loss in the somatic gonad. As a consequence, supernumerary proximal cells are produced at the expense of most distal cells. For instance, malfunction of the Wnt pathway resulted in extra anchor cells (AC, which was generated from the more proximal cell lineage that never received Wnt signaling) at the expense of other somatic gonad lineages. 
A
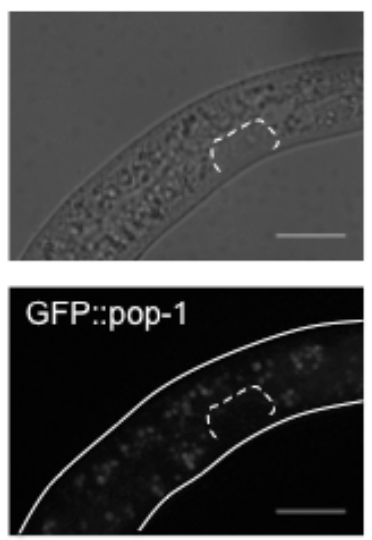

B
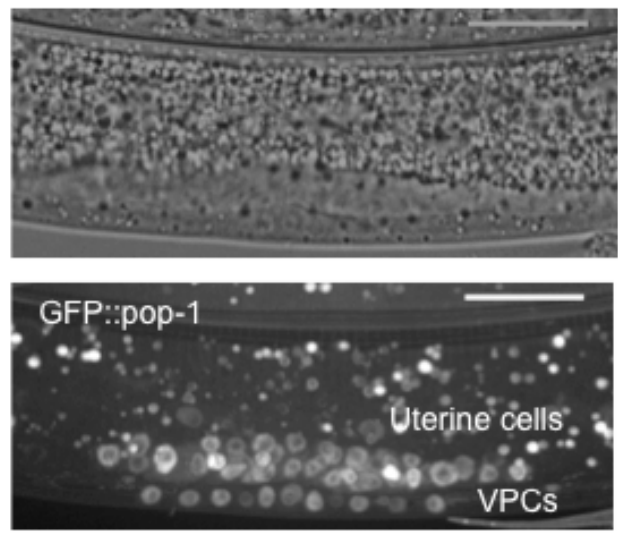

Figure 3.26. Expression of GFP::POP-1 from transgene q/s74 was not detected before L3. A. L1 showing no GFP::POP-1 expression in gonad (dashed lines). B. Vulval precursor cells (VPCs) and uterine cells expressing GFP::POP-1 from L3 larva. Scale bar: $20 \mu \mathrm{m}$.

To analyze whether extra anchor cells are produced in fzr-1(sal19), as indirect evidence of defective Wnt signaling, we introduced the AC reporter zmp-1::GFP (Inoue et al., 2002) in fzr-1(sal19) worms. Extra anchor cells were never detected (Fig. 3.27).
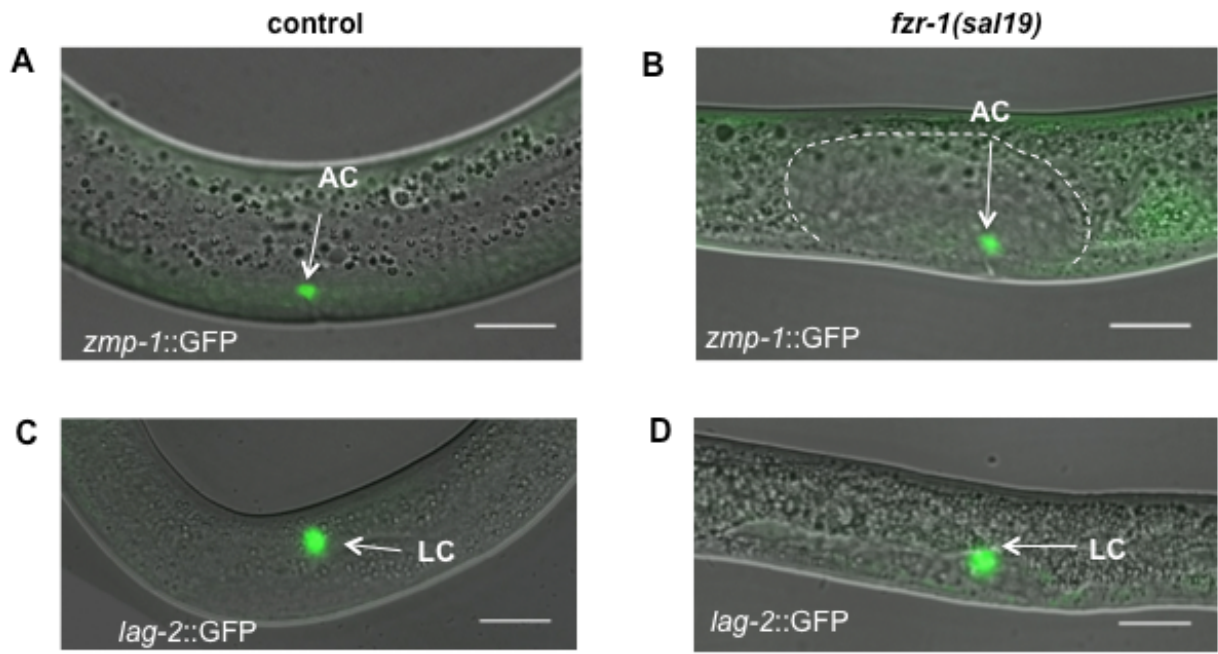

D

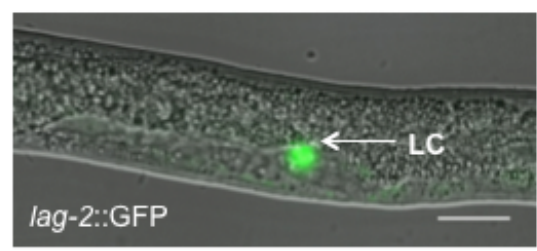

Figure 3.27. fzr-1(sal19) hermaphrodites produce one anchor cell (AC) and fzr-1(sal19) males produce one linker cell (LC). A. Control strain PS3239 expresses zmp-1::GFP transgene, which marks AC during L3 $(n=46)$. B. fzr-1(sal19); zmp-1::GFP produces one AC $(n=68)$. Non-extended gonad is outlined. ACs were analyzed in synchronized L3 larvae. C. LC could be observed with lag-2::GFP till L4 $(n=30)$. D. fzr-1(sal19); lag-2p::GFP males produce one LC $(n=10)$. Scale bar: $20 \mu m$.

Wnt pathway also regulates male somatic gonad development. As its hermaphrodite counterpart, male DTCs functions as stem cell niches, but they 
do not have a leader function, which relies on Linker cell (LC). Wnt male mutants produce extra LC. The LC could be observed until the L4 stage as a rounded cell that positively expresses lag2p::GFP reporter. We never found extra LC in fzr-1(sal19);lag2p::GFP; him-6 male worms, supporting a functional Wnt pathway in fzr-1(sal19) worms again (Fig 3.27).

The non-canonical Wnt-asymmetry pathway is also involved in other asymmetric division, leading to different fates between both daughter cells, such as the seam cells. During larval development, seam cells undergo asymmetrical divisions as Z1 and Z4 did. Seam cell asymmetric divisions rely on the same Wnt components that participate in the somatic gonad. However, seam cell divisions follow the anterior-posterior (A-P) axis instead of D-P. So, after a seam cell divides, the anterior daughter (which does not receive Wnt signal), differentiates into a hypodermal cell. The posterior daughter receives the Wnt signal and maintains its seam identity. When the Wnt pathway is impaired, the number of seam cells dramatically decreases (Gleason \& Eisenmann, 2010). We have scored the number of seam cells in wild type and fzr-1(sal19) adults, using scm-1p::GFP as a reporter for seam cells(Fig. 3.28).

A

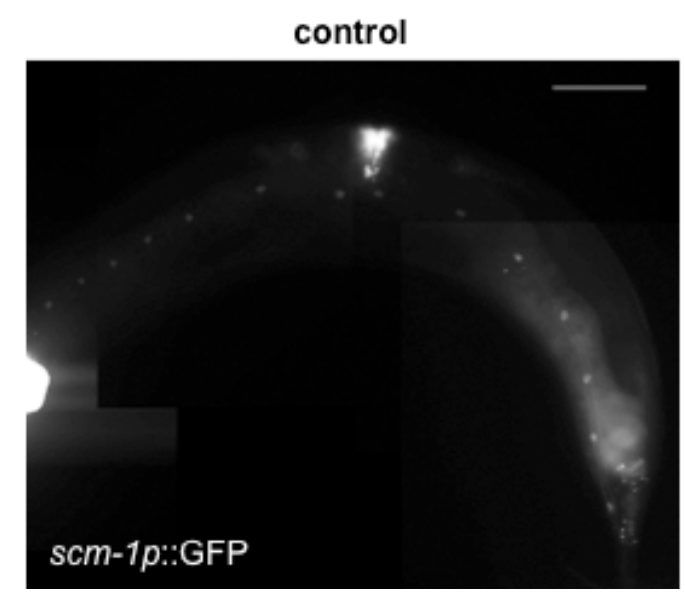

B

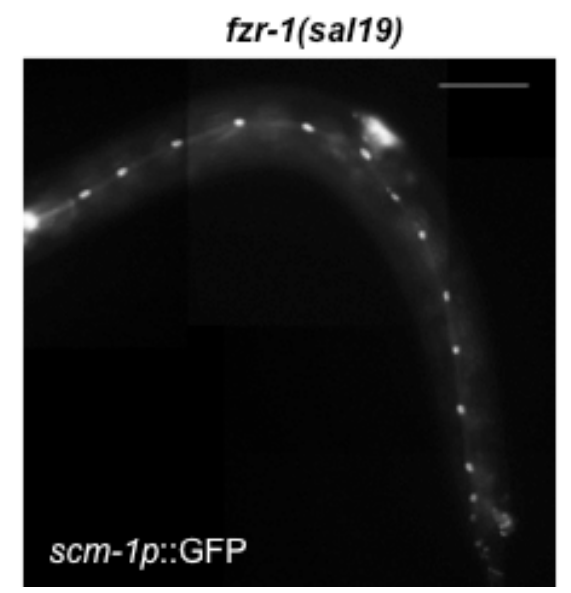

Figure 3.28. seam cells are not affected in fzr-1(sal19). Representative images of control strain RG733 expressing seam cell reporter scm-1p::GFP (A) and fzr-1(sal19);scm-1p::GFP (B). In both cases, more than ten young adult worms were analyzed. Scale bar: $50 \mu \mathrm{m}$.

We did not find significant differences between controls and fzr-1(sal19) regarding the seam cell number. This result indicated that the asymmetric-wnt pathway worked correctly during seam cell division in worms lacking FZR-1. 
In summary, although we cannot discard that the Wnt pathway in Z1 and Z4 was affected in the absence of FZR-1, we have shown pieces of evidence supporting the idea that Wnt pathway was operative in fzr-1(sal19) worms, and that most likely, the impairment in DTC formation observed in hermaphrodites was unrelated to defective Wnt pathway.

\subsection{The absence of DTCs is a consequence of cell lineage alterations in fzr-1(sal19)}

Distinct reasons could cause a lack of DTCs in fzr-1(sal19) adults. One possible reason is an incomplete DTC cell fate acquisition by Z1aa and Z4pp. This lack of completeness could be affecting the expression of some genes, including lag-2. In this case, despite cells that acquire the DTC fate, they were non-functional to sustain germline. Besides, it was impossible to detect these cells because we were using a lag2p::GFP reporter. A second explanation is that Z1aa and Z4pp differentiate into cell types other than DTCs. Finally, a third manner of explaining this absence assumes that DTCs die during the differentiation process in the absence of FZR-1 function.

To address some of these possibilities, we have constructed a lineage tracing system for DTC. It consists of a bipartite transgene expression system (Fig. 3.29). We introduced this bipartite reporter system under the control of Phlh-12 in fzr-1(sal19)/mIn1 worms (Fig. 3.30).

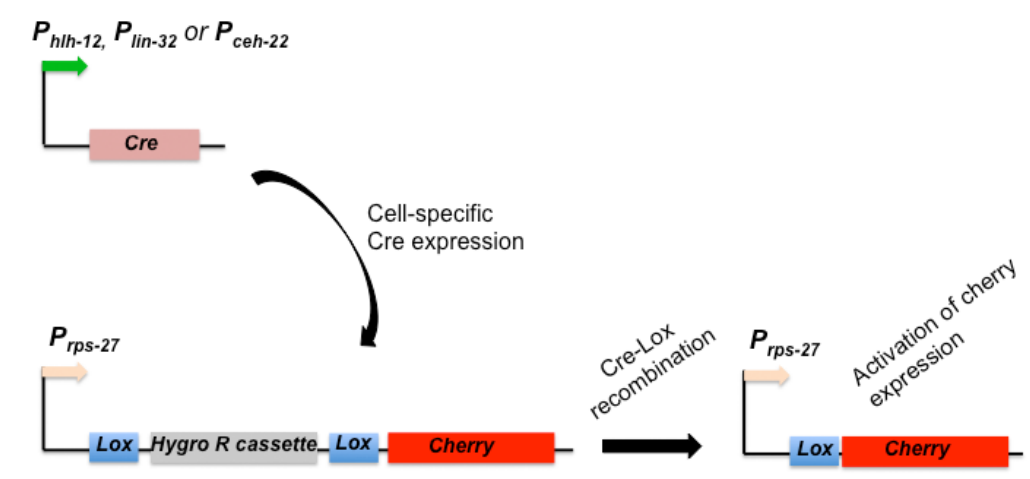

Figure 3.29. Schematic representation of a bipartite transgenic system cell lineages with cherry (Px::cre; rps-27p::lox::hygro::lox::cherry) . In the first part, a Cre recombinase is expressed under the control of $h / h-12$, lin-32 or ceh-22 promoters. When activated, Cre induces lox sites recombination, excising Hygro cassette and leaving cherry in frame with rps-27 promoter. 


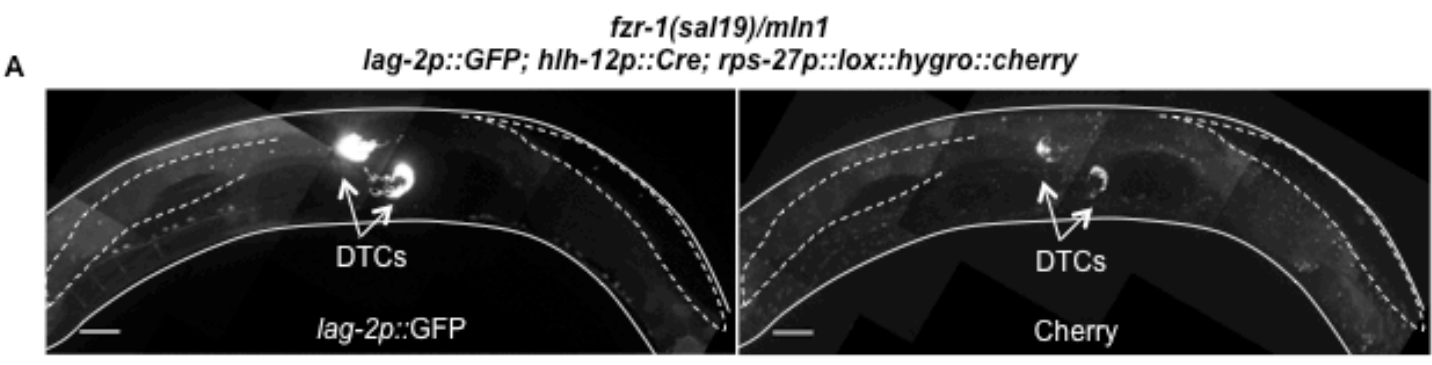

B

fzr-1(sal19)
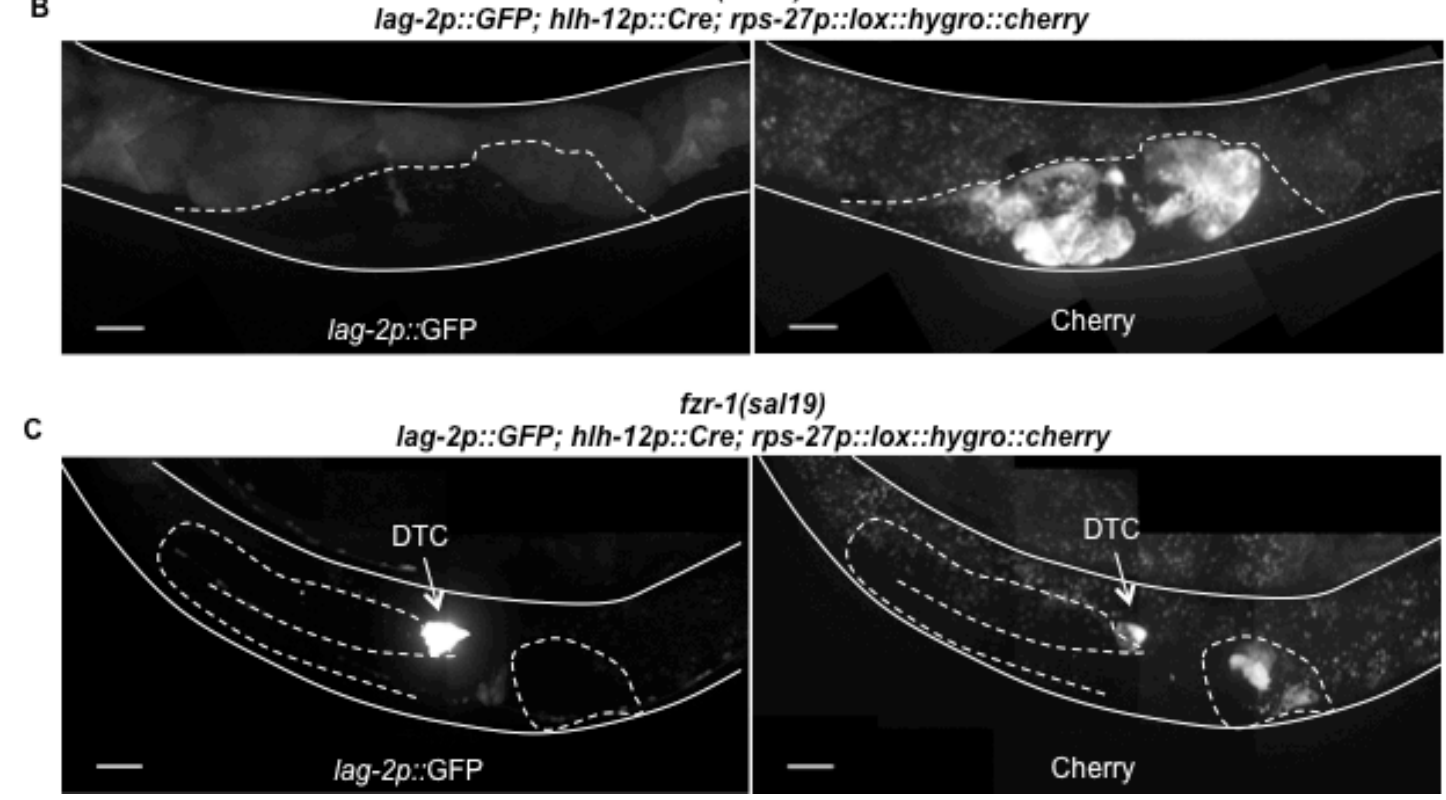

Figure 3.30. fzr-1(sal19) gonads that miss DTCs express Cherry marker for DTC cell lineage A. Extended gonads of control fzr-1(sal19)/mln1 contain two DTCs that express the reporter lag-2p::GFP. Bipartite transgenic system marks specifically both DTCs with Cherry. B. Non-elongated gonads of fzr1(sal19) which miss lag-2p::GFP signal from DTCs are positive for Cherry expression. C. The scarce fzr1(sal19) worms that elongate one gonadal arm show a clear DTC expressing both GFP and cherry. The other half of the gonad, which does not have a DTC (no lag-2p::GFP signal), contain many Cherry-positive cells. Worms are outlined with white lines. Gonads are outlined with dashed lines. Scale bar: $20 \mu \mathrm{m}$.

We have observed than in heterozygous animals, a bright red signal was observed, associated to lag-2:GFP signal. Strikingly, in homozygous fzr1(sal19) lacking lag-2:GFP fluorescence, it was possible to observed a cluster of cells showing red fluorescence in the abnormal gonads (Fig. 3.30B). For that rare fzr-1 (sal19) worms showing one well-formed gonad, we have found cherry fluorescence exclusively associated to DTC (assessed by lag-2::GFP signal) in that gonad, while the other abnormal gonad with no lag2::GFP signal (i. e. not producing DTC) showed the cell cluster mentioned above (Fig. 3.30C).

Careful analysis of the morphology of the cell cluster expressing Cherry in fzr-1(sal19) worms indicated that some of them extended reticulated 
processes that resembled those present on sheath cells. Moreover, other clusters presented and accordion-like structure characteristic of spermatheca (Fig. 3.31).

\author{
fzr-1(sal19); \\ hlh-12p::Cre; rps-27p::lox::hygro::cherry
}

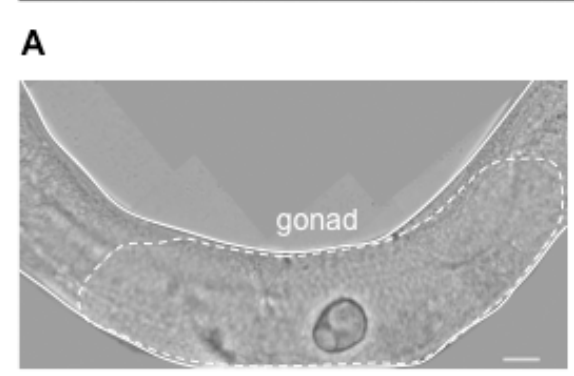

B
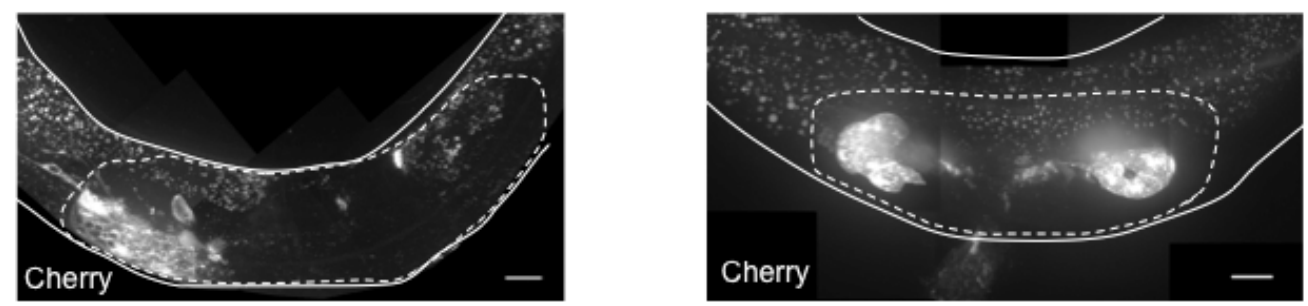

Figure 3.31. Non-DTC cells expressing Z1aa/Z4pp reporter in fzr-1(sal19) gonads resemble sheath and spermatheca cells. A \& B. Upper images show non-extended gonads of fzr-1(sal19) (bright field). A. In lower image, cells that express Z1aa/Z4pp reporter (h/h-12p::cre; rps-27p::lox::hygro::lox::cherry) exhibit a sheath cell-like morphology. B. In lower image, clusters of cells expressing Z1aa/Z4pp reporter present an accordion-like structure, as spermatheca cells. Worms are outlined with white lines and gonads with discontinuous lines. Scale bar: $20 \mu \mathrm{m}$.

This observation suggested that the cell destined to be DTC appears to acquire a distinct cell fate. The appealing possibility prompted us to analyze whether these cells expressing Cherry also expressed markers of sheath cells. We have used lim-7::GFP, which marks 8 of the 10 total sheath cells present per gonad (the two more proximal sheath cells do not express this marker) (Voutev et al., 2009). Encouragingly, we have found a partial overlap between the cherry signal and the lim-7::GFP fluorescence (Fig. 3.32). We noticed that the pattern of expression of lim-7::GFP in $f z r-1$ (sal19) worms is highly disorganized, which can be explained because the organization of sheath cells occurred on a well-developed germline and the germline from $f z r-1$ (sal19) worms is malformed. Furthermore, we analyzed if cells expressing cherry also express spermatheca markers. To address this possibility, we used the sth$1 p:: G F P$ reporter, which marks spermatheca cells (Bando et al., 2005), and we 
have also found an overlap between cherry and sth-1p::GFP signals (Fig 3.33). The sth-1p::GFP signal also describes an irregular pattern in $f z r-1$ (sal19) and partial overlapping. However, the lack of total overlap between sth-1p::GFP or lim-7::GFP signal and cherry signal can be explained keeping in mind that during somatic gonad formation, a cluster of blast cells with different origins (among them the sister cell from DTC, Z1ap or Z4ap) produced both sheath cells and spermatheca.. Altogether, these results can be explained by assuming that in the absence of FZR-1 function, the cell destined to be DTC (Z1aa) acquires the cell fate of its sister (Z1ap) and therefore was used to produce sheath cells and spermatheca. 
Control

(lim-7p::GFP; hlh-12p::Cre; rps-27p::lox::hygro::/ox::cherry)

A

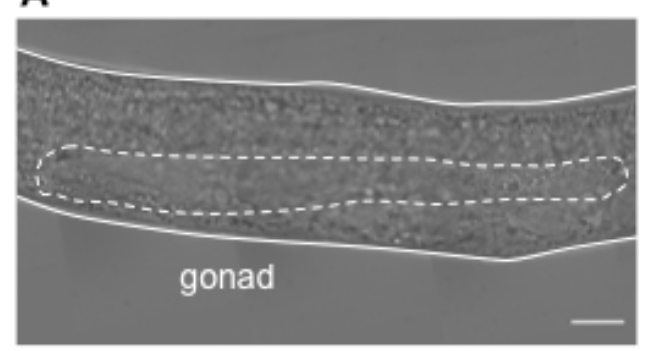

B

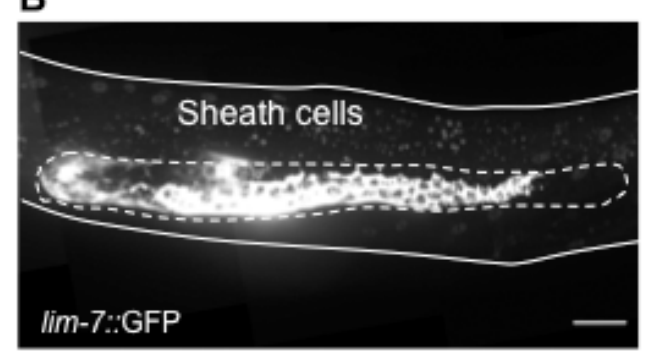

C

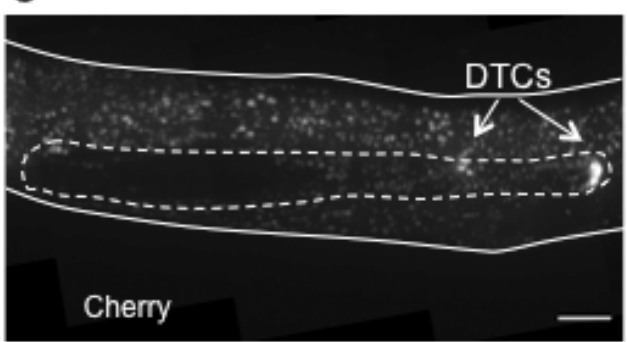

D

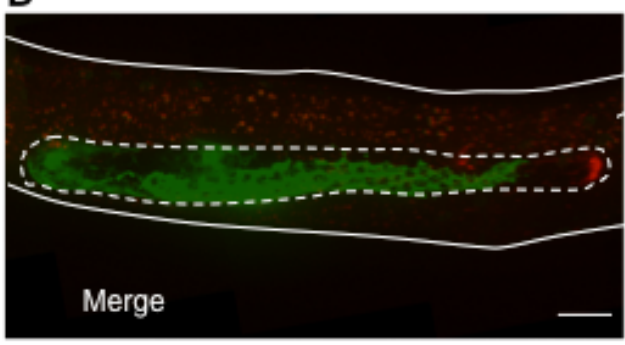

fzr-1(sal19)

(lim-7p::GFP; hlh-12p::Cre;

rps-27p::/ox::hygro::/ox::cherry)

E
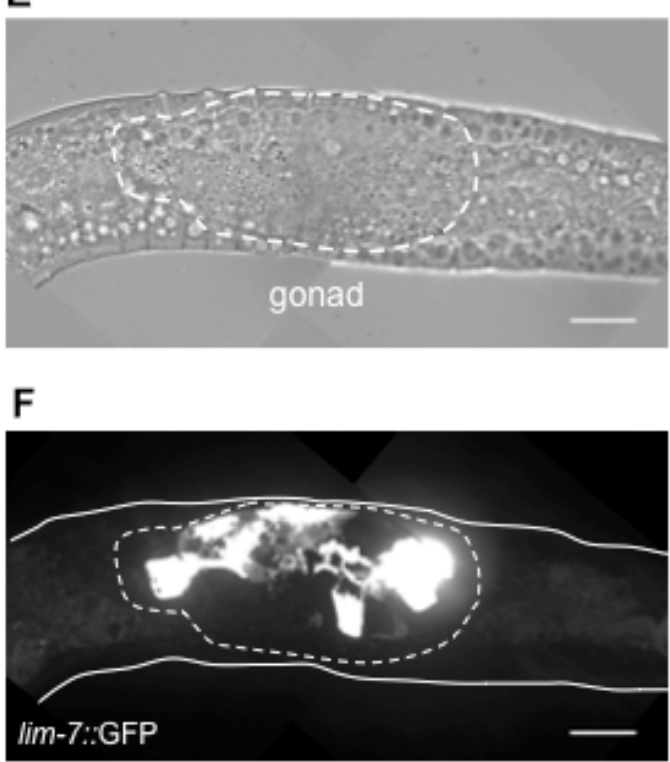

G

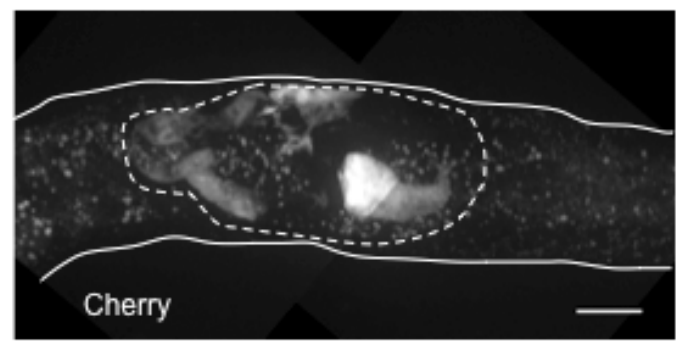

H

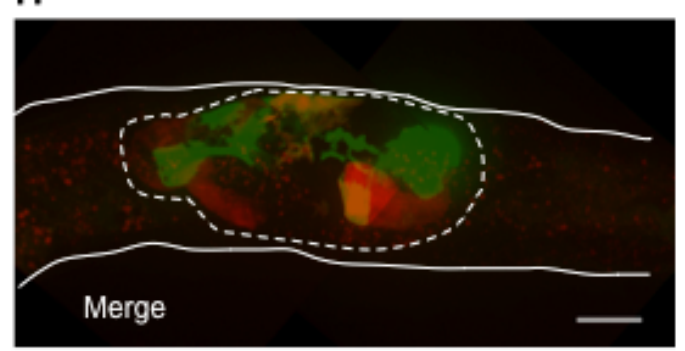

Figure 3.32. Signal from $Z 1$ aa/Z4pp reporter partially overlaps with sheath cell reporter lim-7::GFP in fzr-1(sal19).A-D. Control strain expressing Z1aa/Z4pp reporter (h/h-12p::cre; rps27p::Iox::hygro::/ox:::cherry) and sheath cell reporter lim-7::GFP. Only one gonadal arm is shown. A. Control gonad under bright field. B. Sheath cells, which express lim-7::GFP reporter, are wrapping the germline. C. Expression of Z1aa/Z4pp reporter is activated in DTCs. D. Merge from Z1aa/Z4pp reporter and sheath cell reporter lim-7::GFP shows no overlap between both signals. E-F. Non-extended gonad of fzr-1(sal19) expressing Z1aa/Z4pp reporter (h/h-12p::cre; rps-27p::lox::hygro::Iox::cherry) and sheath cell reporter lim-7::GFP. E. fzr-1(sal19) malformed gonad under bright field. F. Sheath cells are clustered within non-extended gonad of fzr-1(sa/19). G. Z1aa/Z4pp reporter is expressed throughout the gonad of fzr1(sal19). H. Signal from sheath cell reporter lim-7::GFP and Z1aa/Z4pp reporter partially matches. White lines delimit worm's body. Discontinuous lines outline gonadal arm in control and entire gonad in fzr1(sal19). Scale bar: $20 \mu \mathrm{m}$. 
Control

(sth-1p::GFP; phlh-12::Cre; rps-27p::lox::hygro::cherry) fzr-1(sal19)

sth-1p::GFP; phlh-12::Cre;

rps-27p::/ox::hygro::cherry

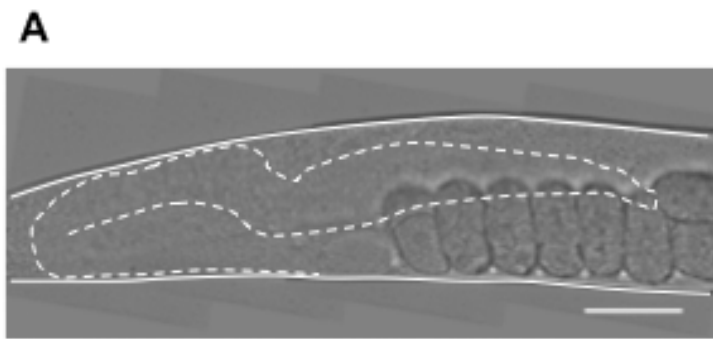

E

B

F
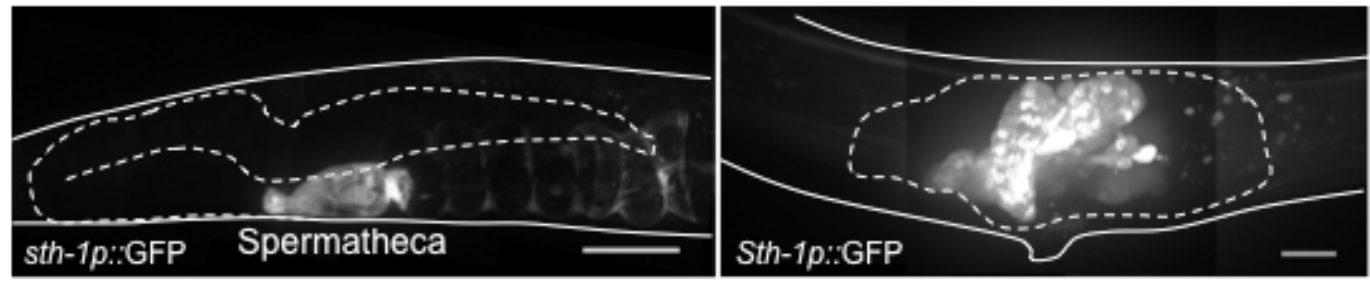

C

G
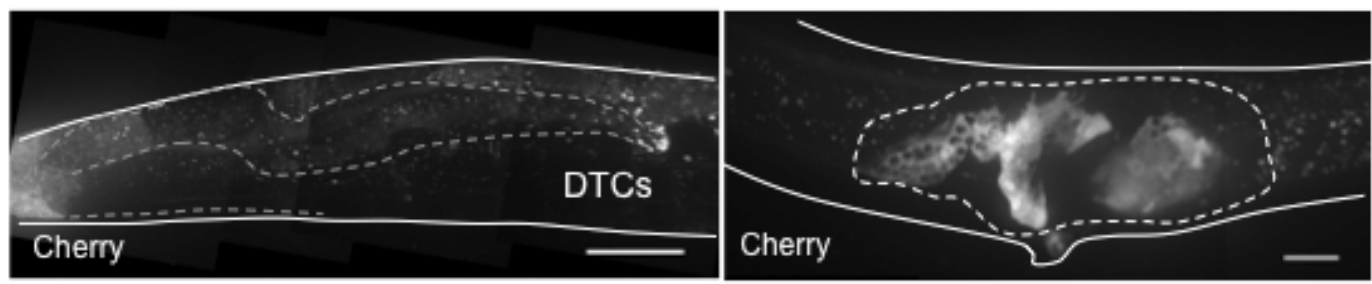

D

H
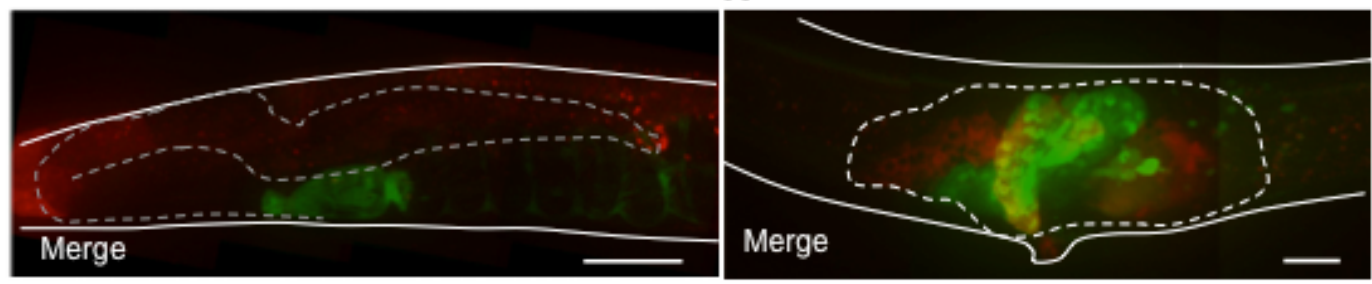

Figure 3.33. Signal from spermatheca reporter sth-1p::GFP partially overlaps with Z1aa/Z4pp reporter in fzr-1(sal19). A-D. Control strain expressing spermatheca reporter sth-1p::GFP and Z1aa/Z4pp reporter (hlh-12p::cre; rps-27p::lox::hygro::lox::cherry). Only one gonadal arm is shown. A. Bright field of control gonad. B. sth-1p::GFP reporter is expressed in spermatheca. C. DTCs activate the expression of Z1aa/Z4pp reporter. D. Merge from Z1aa/Z4pp reporter and spermatheca reporter sth$1 p:: G F P$ shows no overlap. E-F. Non-extended gonad of $f z r-1$ (sal19) expressing Z1aa/Z4pp reporter ( $h / h-$ 12p::cre; rps-27p::lox::hygro::lox::cherry) and spermatheca reporter sth-1p::GFP. E. fzr-1(sal19) malformed gonad through bright field. F. sth-1p::GFP expressing cells are located in the centre of $f z r-1$ (sal19) gonad. G. Expression of Z1aa/Z4pp reporter is spread along the gonad of $f z r-1(s a / 19)$. H. Signal from spermatheca reporter and Z1aa/Z4pp reporter partially matches. White lines delimit worm's body. Discontinuous lines outline gonadal arm in control and entire gonad in fzr-1(sal19). Scale bar: $20 \mu \mathrm{m}$. 


\subsection{Expression of $f z r-1$ in $Z 1 a a / Z 4 p p$ restores DTC production in fzr-1(sal19)}

The capability to promote exclusively the expression of a specific gene (i. e. cherry) in the cell destined to be DTC using the described bipartite system has allowed us to address whether the role of FZR-1 during the differentiation of DTC was cell-autonomous. For that, we have replaced in our bipartite system the cherry encoding sequences with the fzr-1 ORF. Once introduced in fzr1(sal19) worms, we can promote the exclusive expression of $f z r-1$ in Z1aa and Z4pp cells to address whether they complement the observed defects. In agreement with a cell-autonomous role, we have observed tha fzr-1(sal19) worms carrying this system were fully rescued in its ability to produce DTCs (Fig 3.34).

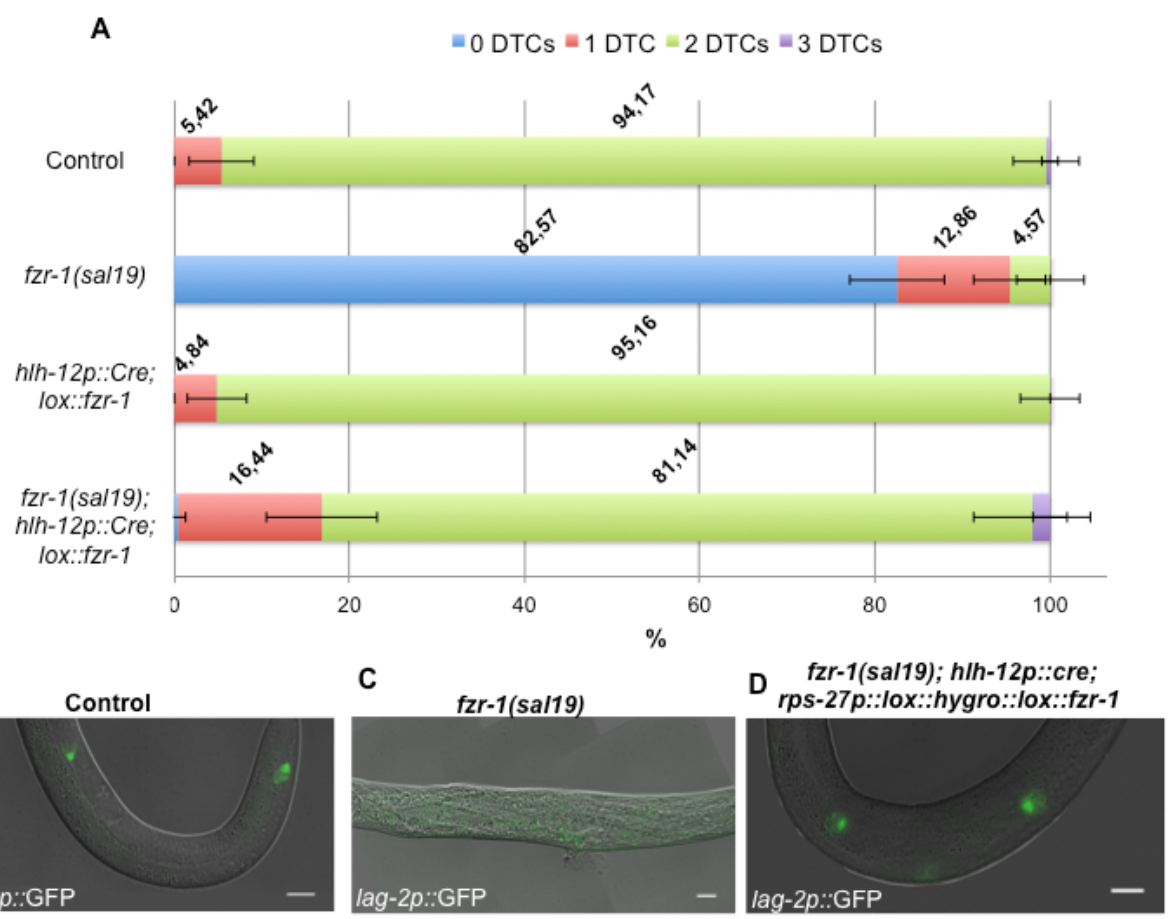

Figure 3.34. Adaptation of Z1aa/Z4pp reporter system to express fzr-1 restores DTC production in fzr-1(sal19). fzr-1 expression was directed by the bipartite system transgene hlh-12p::cre; rps27p::lox::hygro::lox::fzr-1 (hlh-12p:::cre; lox::fzr-1), that was adapted from Z1aa/Z4pp reporter (hlh-12p::cre; rps-27p::/ox::hygro::lox::cherry) A-D. DTCs were observed with lag-2p::GFP reporter A. hlh-12p::cre; lox::fzr-1 restores DTC production in fzr-1(sal19). DTCs were counted under a dissecting scope. Bars represent the mean of two independent experiments with three replicas each ( $n=40$ worms per replica). Error bars symbolize SD. B. Control strain JK2049 containing 2 DTCs C. fzr-1(sal19) devoid of DTCs D. fzr-1(sal19); hlh-12p::cre; lox::fzr-1 produces two DTCs. Scale bar, $20 \mu \mathrm{m}$. 


\subsection{The differences in cell fate between Z1aa and Z1ap are not attributable to different levels of FZR-1}

Our previous results lead to a working hypothesis explaining the cell fate acquisition of Z1aa (Z4pp): in the presence of FZR-1, Z1aa (Z4pp) acquires the DTC fate, while its absence induced presumably the SS sister fate. Cell fate changes between both sisters have been described previously. Mutations in the transcription factors nhr-25 and bet-1 (Asahina et al., 2006, Shibata et al., 2014) cause extra DTCs production that arose from converting the posterior cell (Z1ap) into DTC. We have reasoned that if the presence of FZR-1 was required to acquire the DTC fate, then in fzr-1(sal19) worms, the down-regulation of these transcription factors should not result in the production of extra DTC. We have silenced nhr-25 and bet-1 in fzr-1(sal19) and control worms carrying lag2p::gfp, and in agreement with our educated guess, we have found that no DTCs were present (we were expecting at least one per gonad, arising from the sister cell Z1ap) (Appendix).

Our working hypothesis led us to wonder whether differences in FZR-1 levels could explain the distinct cell fates between Z1aa and Z1ap (i. e. FZR-1 was present in Z1aa but absent in Z1ap). To address that question, we have tried to equalize the levels of FZR-1 in both cells, hoping that the expression of FZR-1 in Z1ap will direct its fate towards DTC (i. e. producing extra DTC). For that, we have directed the expression of fzr-1, using the bipartite system, described in the previous section, coupled to Plin-32 promoter (which promotes the Cre-mediated excision in Z1a/Z4p and therefore, constitutive expression of fzr-1 in their descendants) instead of the Phlh-12 (that promotes excision exclusively in Z1aa). We had found that when this system was activated in N2 worms, no extra DTC was obtained, suggesting that additional controls aside the expression of fzr-1 could be responsible for the proposed role of FZR-1 in cell fate acquisition in these cell types. 


\subsection{A putative phospho-null version of fzr-1 (fzr-1(8A)) yields extra DTCs}

Abounding in the idea that FZR-1 activity could determine the distinct cell fate of the daughters from $\mathrm{Z} 1 \mathrm{a}(\mathrm{Z} 4 \mathrm{p})$, we wondered which kind of regulation (aside from gene expression) could be responsible for this differential activity of FZR-1 between the two sister cells. In other systems, Cdh1/Fzr1 is controlled by two non-excluded manners: either by the binding of the pseudo-substrate inhibitor Emi1/Rca1 (Miller et al., 2006) or by Cdk-dependent phosphorylation. While no homolog has been described for Emi1 in C. elegans, the phosphorylation sites by CDK in FZR-1 were described a few years ago in an in vitro phosphorylation assay using CYD-1/CDK-4 (The et al., 2015).

Modulation of FZR-1 activity by the CDK-mediated phosphorylation is an attractive hypothesis to explain the distinct cell fate of the daughters from Z1a/Z4p. It has been proposed that the sister cell submitted to final DTC differentiation, Z1aa, attached a low level of CDK activity. In contrast, the sister cell destined to produce somatic gonad blast cells (Z1ap) remains quiescent and eventually re-enters the cell cycle, and it maintains a high-medium level of CDK activity (Fujita et al., 2007). It could be well that these differences in CDK activity resulted in differences in FZR-1 activity. In that case, the expression of a non-phosphorylatable fzr-1 allele will disable the ability of CDK to inhibit FZR-1, and therefore it could be predicted that the two daughters from Z1a will be committed to the DTC fate (i. e. the worms will have extra DTC).

Since previous observations concerning the expression of nonphosphorylatable alleles of Cdh1/Fzr1 in human cell cultures suggested defects in cell cycle progression, we have designed a conditional system, based on the use of a tetracycline-controlled ribozyme (Wurmthaler et al., 2019) to enable the expression of the non-phosphorylatable fzr-1 allele at will. Briefly, the sequence encoding the ribozyme was inserted at the 3' UTR of the gene of interest where it self-cleaves the mRNA, precluding its translation and consequently producing no protein. After tetracycline addition, the antibiotic binds to a ribozyme, disabling self-cleavage and enabling mRNA to be translated and thereby protein production (Fig 3.35) .To prove the suitability of this system for conditional expression of $f z r-1$, we have constructed an $f z r-1$ transgene under the ribozyme 
control (fzr-1::rbz) to be inserted at chromosome IV using the Mos system (Fig 3.35). Finally, by crosses, we have introduced this insertion in $f z r-1(s a / 19) / m / n 1$ worms. We segregate homozygous fzr-1(sal19) worms carrying the fzr-1::rbz insertion, and grown in plates with or without $10 \mu \mathrm{M}$ tetracyclin. We have observed that fertility was restored in $f z r-1$ (sal19) fzr-1::rbz worms only when tetracycline was added.

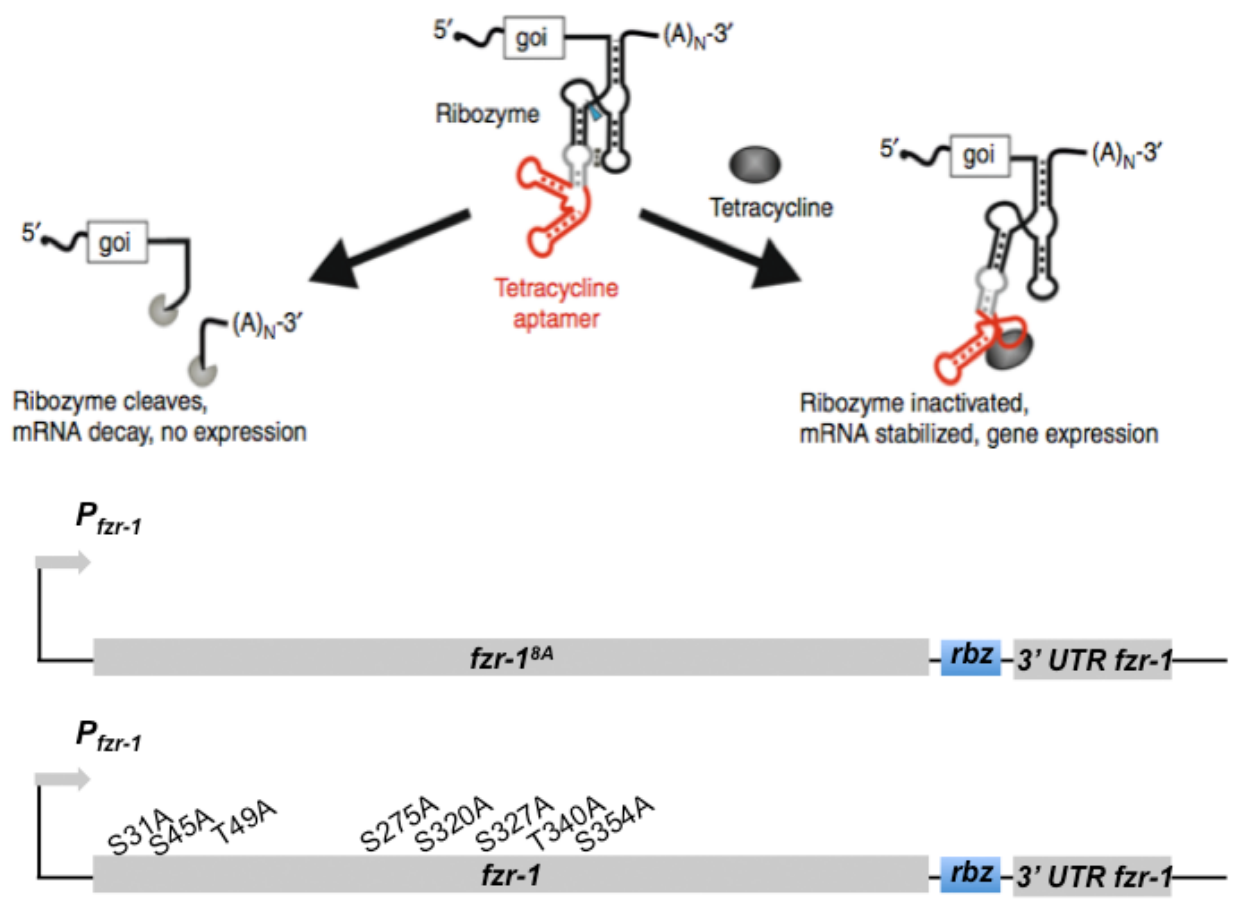

Figure 3.35. Putative phospho-null version of fzr-1 (fzr-1(8A) expressed under the control of Ribozyme-tetracycline based system. Ribozyme-tetracycline based system for inducible expression was adopted to generate inducible transgenes expressing $f z r-1$ and $f z r-1 / f z r-1(8 A)$. Ribozyme sequence is located between $f z r-1 / f z r-1(8 A)$ ORF and 3' UTR. In absence of tetracycline, $f z r-1 / f z r-1(8 A)^{\prime}$ is transcribed followed by ribozyme, which self-cleavages, generating an unstable mRNA that is not translated. Tetracycline inactivates ribozyme and allows $f z r-1 / f z r-1(8 A)$ to be expressed.

We have synthesized a non-phosphorylatable $f z r-1$ allele in which the 8 described CDK-phosphorylation sites (either Ser or Thr) were substituted with Ala residues ( $f z r-1 / f z r-1(8 A))$ and also carrying the above-described ribozyme in its 3' UTR. This mutant allele was introduced at chromosome IV using the Mos system. We have obtained heterozygous $\mathrm{fzr}-1$ (sal19)/mIn1 worms carrying lag2p::gfp and the phospho-null $f z r-1$ transgene $(f z r-1(8 A):: r b z)$ or the wild-type version (fzr-1::rbz). We have segregated $f z r-1(s a / 19)$ in the presence of tetracycline and analyzed the number of DTC in mutant and control worms. 
Encouragingly, we have observed that the expression of the phospho-null fzr-1 resulted in the formation of extra DTCs, which were never observed in the control strain carrying the fzr-1 wild-type version (Fig 3.36).

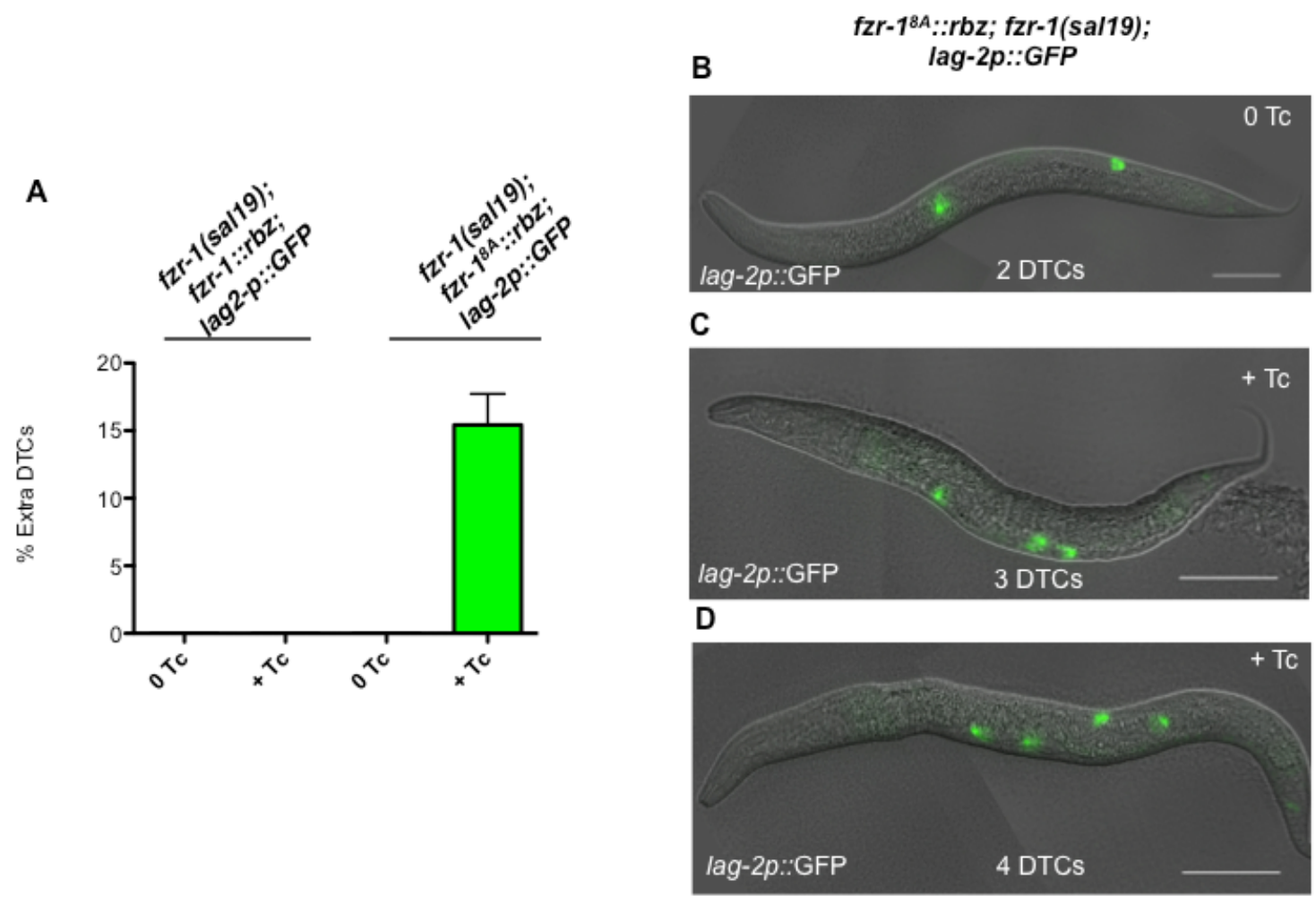

Figure 3.36. Putative phospho-null version of $f z r-1$ (fzr-1(8A)), insensible to inhibitory cdk phosphorilation, renders extra DTCs. A. $f z r-1:: r b z$ and $f z r-1(8 A):: r b z$ were included in $f z r-1$ (sal19); lag$2 p:: G F P$ background to analyze DTC production. Control strain $f z r-1:: r b z$; fzr-1(sal19); lag-2p::GFP does not yield extra DTC without tetracycline $(0 \mathrm{Tc})$ nor after induction with tetracycline $(+\mathrm{Tc})$. On the other hand, in $f z r-1(8 A):: r b z$; fzr-1(sal19); lag-2p::GFP extra DTCs are observed after induction with tetracycline. Synchornized L1 larvae started to feed in media containing $10 \mu \mathrm{M}$ of tetracycline. Columns represent the mean of two independent experiments with three replicas each. Error bars are the SEM. For every replica, DTCs from $n=40$ worms were counted using a dissecting scope. B. Representative image of $f z r-1(8 A):: r b z$ ; fzr-1(sal19); lag-2p::GFP L3 larvae with 2 DTCs that was grown without tetracycline. C \& D. Representative image of $f z r-1(8 A):: r b z$; $f z r-1$ (sal19); lag-2p::GFP L3 larvae with 3 and 4 DTCs grown in presence of tetracycline. Images are overlapings of DIC and GFP. Scale bar, $50 \mu \mathrm{m}$.

\subsection{Most likely, fzr-1(8A) -dependent extra DTC aroses from the differentiation of Z1ap to DTC}

The previous result could be explained assuming that the Z1ap (Z4pa) in the presence of constitutive FZR-1 activity (i. e. not-repressible by CDK) acquires the cell fate of its more anterior sister (DTC). However, alternative 
explanations could also apply to these results. The first alternative is that extra DTC arose from duplication from DTCs itself. For instance, lin-35 mutants showed extra DTCs that arise from the division of already differentiated DTC. Furthermore, this phenotype was enhanced by the presence of $f z r-1$ (ku298). However, we consider this explanation unlikely for different reasons. In the first place, the fzr-1 alleles refractory to CDK-mediated phosphorylation described in other systems opposed G1/S transition, which in our case will preclude further divisions of DTCs. One possibility is to assume that for some reason, in $C$. elegans, the fzr-1 $(8 A)$ allele was hypomorphic, and then was unable to oppose to $\mathrm{G} 1 / \mathrm{S}$ transition. We have found that expression of $f z r-1(8 A)$ in worms already carrying an endogenous copy of a wild-type fzr-1 also resulted in the presence of extra DTC, suggesting a dominance effect more in accord with a gain-offunction than with a hypomorphic allele (Fig 3.37). Indeed, hypomorphic fzr1(ku298) allele alone never renders extra DTCs, only when combined with lin35 (Fay et al., 2002). The second evidence against the duplication of DTC as a source of extra DTC is the observation that when we have analyzed the production of extra DTCs through L3, L4, and adult stages (Fig 3.38A), the percentage of worms with extra DTCs did not change. In fact, in the cases where extra DTC was present, the maximum DTCs observed per worm were four ( 1 from the DTC, and 1 from the sister cell, kin each gonad). In the cases where DTC arises from extra divisions, it could be possible to find more than two extra DTC per gonad (Kostić et al., 2003). Finally, we have found that if the expression of $f z r-1(8 A)$ is induced in L1 larvae, extra DTCs are produced. However, if induction occurred 24 hours later, when worms are around L1-L2 transition, extra DTCs are not detected (Fig 3.38B). Because cell lineages that compose somatic gonad are specified between L1-L2 stages, this observation suggested that extra DTCs are produced during early somatic gonad development; at the time the commitment to distinct cell lineages takes place. 


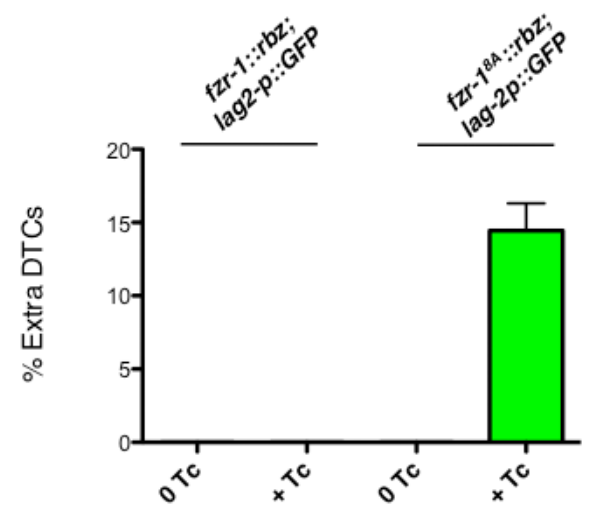

Figure 3.37. Putative phospho-null version of $f z r-1(f z r-1(8 A))$ renders extra DTCs even in the presence of native fzr-1. A. $f z r-1:: r b z$ and $f z r-1(8 A):: r b z$ were included in lag-2p::GFP background to analyze DTC production. Control strain $f z r-1:: r b z ; l a g-2 p:: G F P$ does not yield extra DTC without tetracycline ( $0 \mathrm{Tc}$ ) nor after induction with tetracycline $(+\mathrm{Tc})$. On the other hand, in $f z r-1(8 A):: r b z$;; lag$2 p:: G F P$ extra DTCs are observed after induction with tetracycline. Synchornized L1 larvae started to feed in media containing $10 \mu \mathrm{M}$ of tetracycline. Columns represent the mean of two independent experiments with three replicas each. Error bars are the SEM. For every replica, DTCs from $n=40$ worms were counted using a dissecting scope.

fzr-1(sal19); fzr-1 ${ }^{8 A}:: r b z ;$ lag2p::gfp

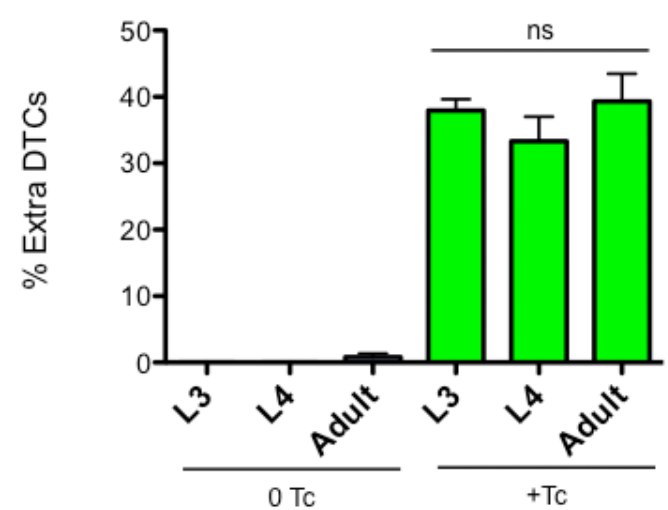

fzr-1(sal19); fzr-1 ${ }^{8 A}:: r b z ;$ lag2p::gfp

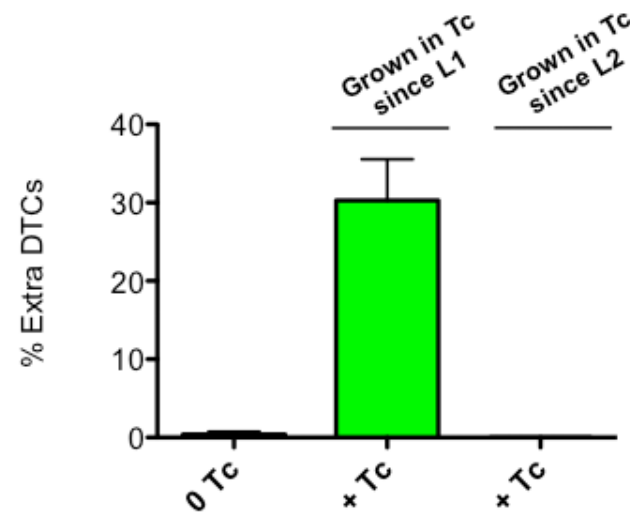

Figure 3.38. Extra DTCs are produced during early somatic gonad development in fzr-1(8A). A. Synchronized $f z r-1(8 A):: r b z$; fzr-1(sal19); lag-2p::GFP L1 larvae started to feed in dishes without and with tetracycline $(10 \mu \mathrm{M}):(0 \mathrm{Tc})$ and $(+\mathrm{Tc})$, respectively. Extra DTCs were counted at L3, L4 and in young adults. The frequency (\%) of worms with extra DTCs was the same throughout the different stages. B. Synchronized $f z r-1(8 A):: r b z$; fzr-1(sal19); lag-2p::GFP L1 larvae started to feed in dishes without and with tetracycline $(10 \mu \mathrm{M}):(0 \mathrm{Tc})$ and $(+\mathrm{Tc})$, respectively. In presence of Tc, worms produced extra DTCs, which were counted once worms reached adulthood. 24h later, L2 worms from control dishes (0 Tc) were changed to new dishes with Tc, but they did not yield extra DTCs. A \& B. In both experiments, columns represent the mean of two independent experiments with three replicas each. Error bars are the SEM. For every replica, DTCs from $n=40$ worms were counted using a dissecting scope. ns: no significant. 
A second alternative explanation is to assume that, for some reason, the presence of the $f z r-1(8 A)$ allele is altering the Wnt pathway, and therefore the distinct cell lineages were misled. It has been described that overactivation of Wnt signaling yields extra DTCs at expenses of AC. We have analyzed the number of $\mathrm{AC}$ in these worms as an indirect readout of the altered Wnt pathway. We have found that worms with extra DTCs always have one anchor cell, suggesting that the Wnt pathway is not affected (Fig 3.39).
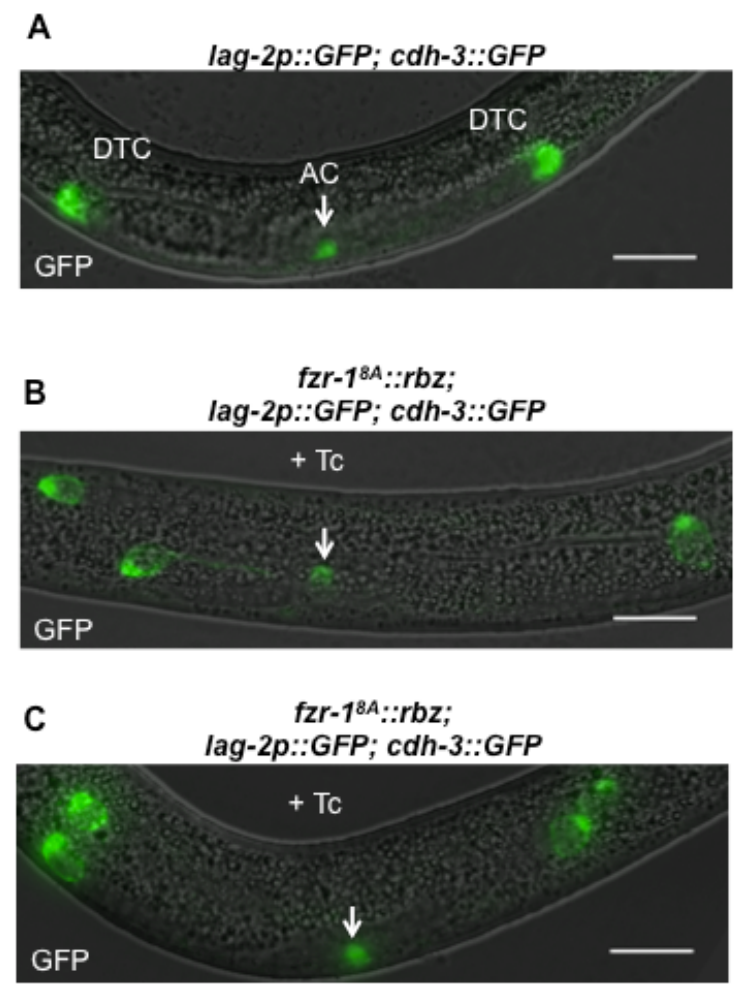

Figure 3.39. $f z r-1(8 A)$ renders extra DTCs that are not produced at the expense of AC. A. Control strain produces two DTC and one AC that are observed with reporters lag-2p::GFP and cdh-3::GFP at L3 stage, respectively. B \& C. Induction of $f z r-1(8 A):: r b z$ with tetracycline yields extra DTCs that do not affect ac production. Arrows point AC. Images are overlapping of DIC and GFP. Scale bar, $20 \mu \mathrm{m}$.

\subsection{The Wnt pathway could be controlling APC ${ }^{\mathrm{FZR}-1}$ activity by regulating CYE-1 asymmetry}

Altogether these previous results strongly support a working hypothesis by which the different levels of CDK phosphorylation of FZR-1 could lead to differential activity of the APC/C ${ }^{\text {FZR-1 }}$ complex. As a consequence, this will result in the degradation of unknown target(s) that determine one or another fate: DTC (low CDK activity, high FZR-1 activity) or somatic gonad blast precursor (high 
CDK activity, low FZR-1 activity). Encouragingly, it has been reported that the Wnt pathway represses cye-1 expression in Z1aa/Z4pp. However, Z1ap/Z4pa, in which the Wnt pathway is not activated, keeps high levels of CYE-1. Moreover, In cye-1 mutants or upon cye-1 RNAi, Z1ap/Z4pa, differentiate into DTCs instead of commit SS precursor fate. On the contrary, the ectopic expression of cye-1 leads to the absence of DTCs (Fujita et al., 2007).

Altogether, these observations prompted us to think that the Wnt pathway could determine asymmetric levels of CYE-1 (and hence of CDK activity), which control APC/C ${ }^{\text {FZR-1 }}$ activity. This will result in the distinct cell fates of the daughter cells arising from Z1a division. In this way, in Z1aa/Z4pp, which gives rise to DTCs, CYE-1 levels are kept low. Consequently, FZR-1 would be active due to its hypophosphorylated state. APC/C ${ }^{\text {FZR-1 }}$ would target for proteasomal degradation one or many repressors, activating the process of differentiation into DTCs. In Z1ap/Z4pa, which becomes SS precursors, CYE-1 levels are high and FZR-1 hyperphosphorylated, switching off its activity.

To test this hypothesis, we analyzed several predictions inferred from it. In the first place, as CYE-1 would act upstream of APC/C $\mathrm{C}^{\mathrm{FZ}-1}$, depletion of CYE-1 levels should not result in extra DTCs in fzr-1(sal19). We have RNAi cye-1 in fzr-1(sal19), and, according to our prediction, no effect was observed in the presence of an extra DTC number (Fig 3.40). In the second place, if CYE-1 and $A P C / C^{\text {FZR-1 }}$ were acting in the same pathway, depletion of CYE-1 levels and simultaneous induction of $f z r-1(8 A)$ should not increase the frequency of extra DTC production when compared with depletion of CYE-1 or induction of $f z r-1(8 A)$ separately. Figure 3.41 shows that when combined, silencing of cye-1 and induction of $f z r-1^{8 A}$, non-summatorial increase in the frequency of extra DTC was observed, confirming this second prediction. 


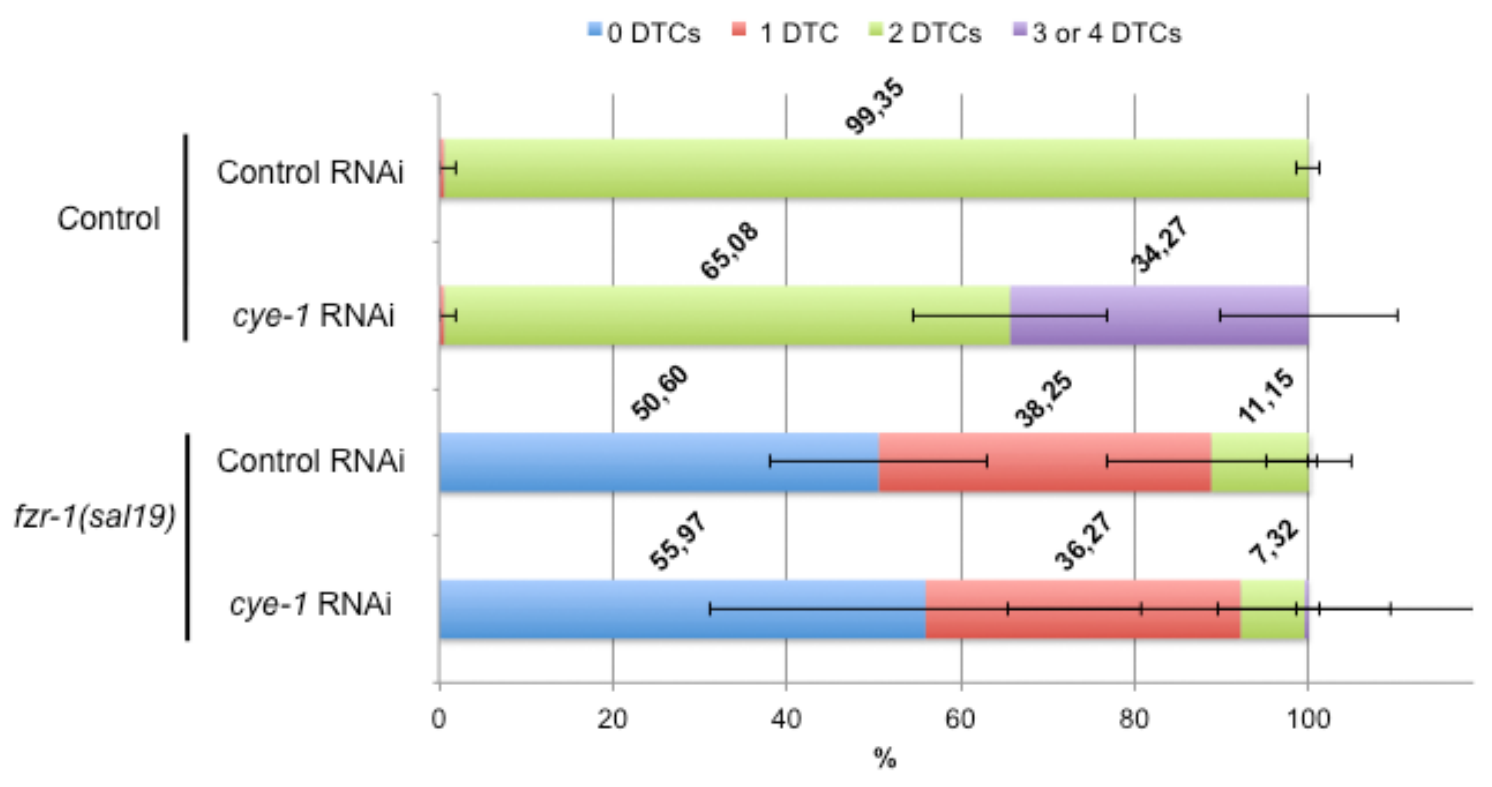

Figure 3.40. Depletion of CYE-1 levels does not restore DTCs production in fzr-1(sal19). After $\mathrm{Z} 1 \mathrm{a} / \mathrm{Z} 4 \mathrm{p}$ divides, $\mathrm{CYE}-1$ is distributed asymmetrically between distal and proximal daughter cells. Proximal Z1ap/Z4pa (SS precursor cells) receives higher doses of CYE-1 than distal Z1aa/Z4pp (DTCs). CYE-1 behaves as a repressor of DTC fate commitment. Depletion of CYE-1 levels makes Z1ap/Z4pa differentiate into DTCs, instead of SS precursors, yielding extra DTCs. We analyzed if DTCs production could be restored in fzr-1(sal19) after lowering cye-1 expression. Experiments were carried out by silencing cye-1 following RNAi feeding method. For every dish, three to four $f z r-1$ (sal19)/mIn1; lag-2p::GFP L4s were grown feeding on dsRNA producing bacteria and let them to produce progeny. DTCs from silenced F1 fzr-1(sal19); lag-2p::GFP were counted using a dissecting scope. JK2049 was used as a control strain. Bars represent the mean of two independent experiments with three replicas each. Error bars symbolize SD. 
A

fzr-1(sal19); fzr-1 ${ }^{8 A} ;$ lag2p:::gfp

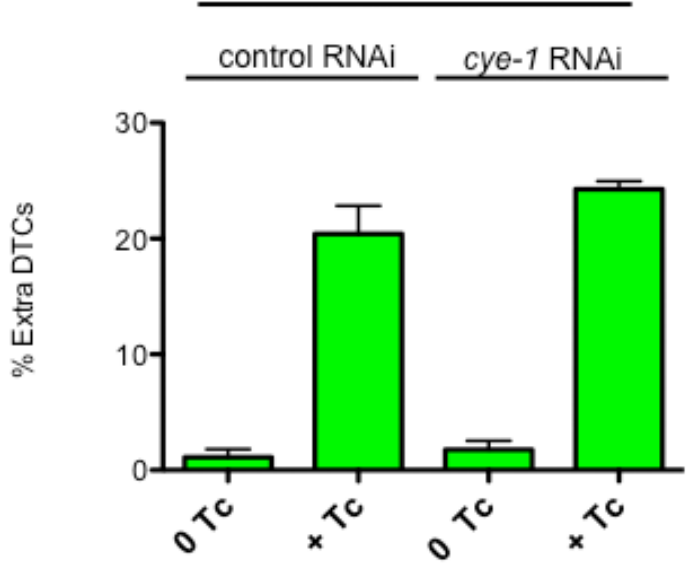

B

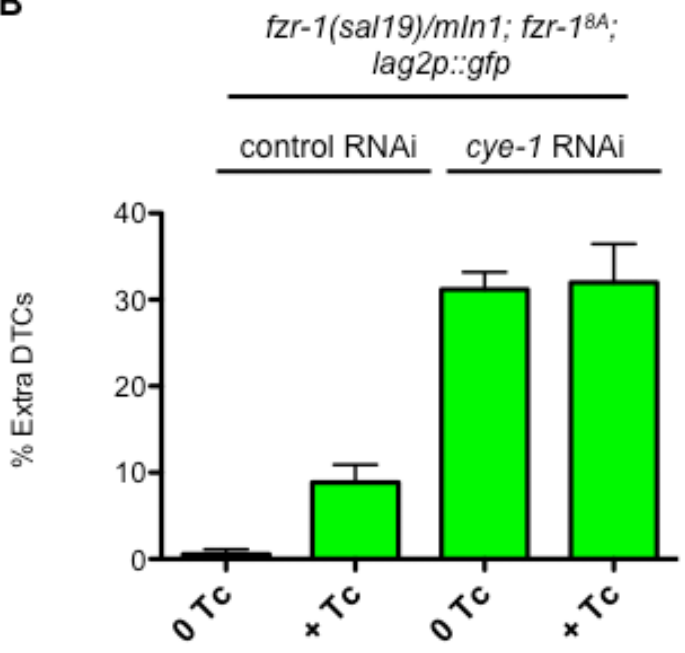

Figure 3.41. Production of extra DTCs is not increased after simultaneous induction of $f z r-1(8 A)$ and depletion of CYE-1. A \& B. For every dish, three to four $f z r-1(s a l 19) / m / n 1 ; f z r-1(8 A):: r b z$; lag$2 p:: G F P$ L4s were grown feeding on dsRNA producing bacteria and let them to produce eggs, which were collected and synchronized. $f z r-1(8 A)$ expression was induced by growing synchronized L1s in RNAi dishes supplied with $50 \mu \mathrm{M}$. DTCs from F1 were counted using a dissecting scope. In both experiments, columns represent the mean of two independent experiments with three replicas each. Error bars indicate SEM. For every replica, DTCs from $n=40$ worms were counted A. DTCs were counted in fzr-1(sal19); fzr1(8A)::rbz; lag-2p::GFP F1. B. DTCs were counted in $f z r-1(s a l 19) / m \ln 1 ; f z r-1(8 A):: r b z ;$ lag-2p::GFP F1. 



\section{DISCUSSION}

Strict coordination between cell division and differentiation is required to develop an organism and tissue homeostasis, among others. Alterations in that coordination could give rise to illnesses such as cancer. Cell division, through the cell-cycle machinery, controls the activation of differentiation processes. Specifically, G1 phase offers an 'opportunity window' to commit a cell differentiation program. In this regard, regulators of $\mathrm{G} 1 / \mathrm{S}$ play an essential role in modulating the connection between cell cycle and differentiation. Among the different G1/S regulators highlights the E3 ubiquitin ligase APC/C with its coactivator Cdh1 (FZR-1 in C. elegans). Apart from its role in cell cycle, APC/C ${ }^{\text {Cdh1 }}$ is gaining attention as a direct link between cell cycle and cell differentiation due to its ability to target directly factors implicated in differentiation. This thesis studied new roles of APC/C ${ }^{\text {FZR-1 }}$ in germline and soma of the model organism Caenorhabditis elegans.

\section{1. $A P C / C^{\text {FZR-1 }}$ targets Polycomb subunit MES-3}

Initial studies from our laboratory demonstrated that $\mathrm{APC} / \mathrm{C}^{\mathrm{FZR}-1}$ was involved in the control of the H3K36 methyltransferase MES-4 through its KEN box motif. MES-4 protein is expressed in distal and proximal regions in the germline, while its levels drop in early pachytene. The depletion of APC/C ${ }^{\text {FZR-1 }}$ activity or mutation of its KEN-box into triple alanine makes MES-4 protein present in the entire gonad, invading pachytene (Rivera-Martín S, 2018). Here, in this Thesis work, we uncovered that APC/C ${ }^{\text {FZR-1 }}$ also targets MES-3.

MES-3 is part of the Polycomb Repressive Complex 2 (PRC2) in C. elegans, although it is not present in other metazoan. PRC2 is an H3K27me2/3 methyltransferase also composed in $C$. elegans by the catalytic subunit MES-2 and accessory subunit MES-6, homologs of Drosophila $E(Z)$ and ESC, respectively (Bender et al., 2004b). Immunostaining of MES-2 and MES-6 showed that both proteins are present throughout the entire gonad (Holdeman et al., 1998). However, MES-3, like MES-4, was absent in early pachytene. Previous studies focused on MES-3 regulation in germline proposed that MES-3 levels drop along pachytene because the repression of its mRNA translation by 
GLD-1, which binds to the 3' UTR of mes-3 (Xu et al., 2001). Here we provide evidence that the absence of MES-3 along pachytene is a combined action of post-transcriptional regulation by GLD-1 and a post-translational control by $\mathrm{APC} / \mathrm{C}^{\mathrm{FZR}-1}$. We believe that degradation of MES-3 led by $A P C / C^{\mathrm{FZR}-1}$ and inhibition by GLD-1 of mes-3 mRNA translation constitutes a double barrier that excludes MES-3 from pachytene. Disruption of either element of this barrier resulted in the invasion of pachytene by MES-3.

\subsection{Non-degradable MES-3(AAA) causes fertility defects that could come from the pachytene invasion of MES-3}

mes-3(AAA) suffers from fertility defects that could be explained, at least in part, by the presence of aberrant embryos within the uterus. We hypothesized that such defects arose from the pachytene invasion of MES-3, which is the most obvious difference observed when we compare the gonad distribution of a non-degradable and control MES-3::GFP fusions. We believe that because the role of PRC2 repressing transcription, this invasion could promote non-scheduled repression of genes. Because in early pachytene many genes required for oocyte and embryo development were transcribed, we propose that some of these genes were repressed and as a consequence oocytes or embryos were non-functional.

The observation that the presence of a non-degradable allele of mes-4, which also invades the early pachytene region, suppresses these fertility defects supports our hypothesis. It has been proposed that one of the roles of MES-4 is to act as a barrier that limits the location of PCR2 to unscheduled loci. It may be well that in worms carrying the mes-3(AAA) allele alone, because the absence of MES-4 in early pachytene region, PRC2 invades ectopic regions. However, in worms carrying mes-3(AAA) and mes-4(AAA) alleles, the presence of MES-4 in early pachytene limits the ability of PRC2 to invade genomics regions. Under this point of view, one of the reasons why these chromatin regulators are controlled by $\mathrm{APC} / \mathrm{C}^{\mathrm{FZR}-1}$ could be related to the necessity to maintain a balance between the activities of PRC2 and MES-4, through simultaneous degradation of MES-3 and MES-4. 
We have tried to add further support to our hypothesis by promoting the invasion of early pachytene region by ways non-dependent on MES-3 degradation by $\mathrm{APC} / \mathrm{C}^{\mathrm{FZR}-1}$. We have reasoned that if mes-3(AAA) fertility defects are originated by the pachytene invasion of MES-3, we would expect that pachytene invasion of MES-3 in mes-3::tbb-2 3'UTR mutants yield similar fertility defects. However, fertility levels of mes-3::tbb-2 3'UTR were close to wild-type fertility levels. Besides, we did not observe defective eggs. Although these could contradict our hypothesis, it is worth mentioning that under $t b b-2$ 3'UTR, the absolute levels of MES-3::GFP were lower than those from an allele carrying mes-3' 3 UTR, and that it could be possible that the absolute levels of MES-3 in this case were too low for promote the above proposed unscheduled invasion of chromatin by PRC2. Supporting this idea, we have found that the mutant allele mes-3(AAA)::tbb-2 3'UTR was strongly alleviated in its fertility defects with respect to mes-3(AAA) allele. It will be necessary to repeat these experiments, choosing a heterologous 3'UTR (not targeted by GLD-1) but leading higher MES-3 levels expression. Alternatively, a more precise approach would be to mutate the predicted GLD-1 binding sites in mes-3' 3 UTR. It is also possible that degradation of MES-3 trough APC/C ${ }^{\mathrm{FZR}-1}$ constitutes the main regulation of mes-3 expression and translational repression of GLD-1 a secondary control. Future research will shed more light on this question.

\subsection{Fertility defects associated with mes-3(AAA) are independent of LIN-35 (DRM/DREAM complex) but partially rescued by lin-15b mutants}

The combined action of Polycomb and MES-4 is counteracted by LIN15B and the transcription factor DRM/DREAM complex, that includes: LIN-35 (Rb), EFL-1/DPL-1(E2F/DP) heterodimer, and Multi-vulva class $B$ core subunits LIN-9,-37,-52,-53 and-54 (Lee et al., 2017). DRM/DREAM and LIN-15B inhibit Polycomb and MES-4 in soma, preventing ectopic expression of germinal genes. On the contrary, the specification of the germinal identity of PGCs requires the repression of maternal DRM/DREAM and LIN15B to activate Polycomb and MES-4. In the germline, DRM/DREAM activity opposes MES-4 methylation. The molecular details regarding the interaction between MES-4 
and DRM/DREAM remain mostly unknown. We expected that fertility defects upon depletion of DRM/DREAM subunit LIN-35 were increased in lin-35; mes$4(A A A)$ double mutants. However, the decay of fertility levels are the same for lin-35 and lin-35; mes-4(AAA). On the other hand, we observed that fertility defects in lin-35 and mes-3(AAA) were additive in lin-35; mes-3(AAA) and can be restored to those of lin-35 in lin-35; mes-3(AAA); mes-4(AAA) triple mutants. Moreover, the percentage of worms containing aberrant eggs in the different mutants does not correlate with fertility levels. Altogether, these results suggested that MES-3(AAA) and MES-4(AAA) are acting independently of DRM/DREAM (Tabuchi et al., 2014). Indeed, previous studies corroborate that in the germline, DPL-1, and EFL-1 act instead of LIN-35 (Chi \& Reinke, 2006). It would be advisable to conduct new fertility assays substituting lin-35 by $d p /-1$ and efl-1.

We also carried out fertility assays for single mutant lin-15B in combination with mes-3(AAA) and mes-4(AAA). In every case, fertility levels are very close to those of lin-15B, although the levels of mes-3(AAA); mes-4(AAA); lin-15B triple mutants are lower than mes-4(AAA); lin-15B, which, in turn, are lower than mes-3(AAA); lin-15B. Strikingly, lin-15B significantly relieves fertility defects of mes-3(AAA). Moreover, the percentage of worms with defective eggs among these mutants highly matches with the fertility results. LIN-15B is associated with HMTs that repress gene expression through methylation of H3K9 (Rechtsteiner et al., 2019). In the germline, dimethylation of H3K9 is detected in the distal region and from late pachytene and depends on the HMT MET-2 (Bessler et al., 2010). The partial recovery of mes-3(AAA) fertility defects in mes-3(AAA); lin-15B could be explained by an overall reduction of $\mathrm{H} 3 \mathrm{~K} 9 \mathrm{me} 2$ in the germline. The classical steps towards repression of gene expression start with H3K27 methylation, a bivalent mark, and continued by H3K9 to achieve permanent and complete gene expression repression. In this regard, Polycomb's possible ectopic activity in pachytene led by MES-3(AAA) could be counteracted through depletion of LIN-15B and the concomitant reduction of $\mathrm{H} 3 \mathrm{~K} 9$ activity. At least, a partial recovery in fertility levels of mes-3(AAA) should be observed in mes-3(AAA); met-2 double mutants to support this hypothesis.

On the other hand, the trimethylation of $\mathrm{H} 3 \mathrm{~K} 9$ is detected along the entire germline. Interestingly, Polycomb, apart from its role in methylation of H3K27, is 
also capable of trimethylate H3K9 (Bessler et al., 2010). It is tempting to think that MES-3 could control the triH3K9 activity of Polycomb. Chromatin analysis of H3K27 and H3K9 methylation mark in mes-3(AAA) will yield useful information in this regard.

\subsection{Loss-of-function of fzr-1 affects germline development only in hermaphrodites}

Severe loss of function mutants of $\mathrm{APC} / \mathrm{C}$ core subunits leads to a onecell stage arrested embryos, masking the study of the roles played by APC/C during post-embryonic development. The use of thermosensitive mutants bypassed that restriction, unveiling the processes in which APC/C takes part along larval development. APC/C mutants that scape from embryonic arrest features uncoordinated movement, protruding/everted vulva, defective male tail, and sterility. These phenotypes are explained by defects in the ventral nerve cord, vulva, rays development, and gonads, respectively. After hatching, waves of mitotic divisions occur in those organs, which are deprived of maternal doses of the particular APC/C subunit. Consequently, cells cease division and yield aberrant organs (Furuta et al., 2000, Golden et al., 2000, Shakes et al., 2003). Nonetheless, if APC/C affects organ development through the cell cycle and/or differentiation remains elusive.

We have obtained a complete loss-of-function allele of $f z r-1, f z r-1$ (sal19). Homozygous $f z r-1$ (sal19) suffer the same developmental defects than mutants in other subunits of APC/C: unc mobility, everted vulva, male tail without rays, and sterility. Homozygous $f z r-1(s a / 19)$ are $M+Z-$, since they come from heterozygous mothers $\mathrm{fzr}-1(\mathrm{sal} / 9) / \mathrm{m} / \mathrm{n} 1$. We thought that maternal load would help homozygous $f z r-1(s a l 19)$ to scape possible embryo arrest. However, the maternal load of $f z r-1$ could not be necessary to reach adulthood, as microinjection of fzr-1 RNAi yields F1 progeny (M-Z-) that develop into sterile adults (Fay et al., 2002). It could not be discarded that minimal doses of $f z r-1$ mRNA or FZR-1 remain and are enough to circumvent possible arrest during development. It is noteworthy that mutants $f z r-1(k u 298)$ and $f z r-1$ (ok380) develop as wild-type, suggesting that even low activity of APC/C ${ }^{\text {FZR-1 }}$ allows appropriated development. 
Sterility defects of fzr-1(sal19) are explained, in part, by malformed gonads that do not elongate, as previously reported after microinjection of $f z r-1$ RNAi (Fay et al., 2002). We determined that aberrant gonads in fzr-1(sal19) stem from the somatic gonad's developmental failures. Specifically, fzr-1(sal19) lacks DTCs, the stem-cell niche that controls germline growth and maintenance. Only a low percentage of cases, $f z r-1$ (sal19) can generate DTCs. When produced, these DTCs fulfill the two essential functions attributed to DTCs: leading of gonadal elongation and maintaining of germ cell pools. The resulting elongated germlines indicate that $A P C / C^{F Z R-1}$ is not necessary for the division of germ cells.

Nonetheless, APC/C is necessary for germ cell division and maintenance, since mutants in core APC/C subunits cause that germ cells to stop to divide prematurely. Accordingly, to previous reports, APC/C with its coactivator FZY-1 (Cdc20) is necessary for proper germ cell division (Kitagawa et al., 2002). However, fzr-1(sal19) germlines are sterile. In best cases, they produced few aberrant eggs that never hatch nor were expelled. These aberrant eggs could stem from defects in the proximal somatic gonad (proximal sheathcells, spermatheca, and/or uterus), from an essential role of FZR-1 during early embryogenesis, or a combination of both.

On the other hand, fzr-1(sal19) males are also sterile despite that they always develop an extended J-shaped gonad. We do not know whether sterility of males arises from defects in germline and/or alterations in mating behavior. The reasons for sterility in hermaphrodites and males will be a matter of future research.

\section{5. $A P C / C^{\text {FZR-1 }}$ determines DTC identity in hermaphrodites}

We mainly focused on the role played by APC/C ${ }^{F R-1}$ during the production of DTCs. We first ruled out that 'DTCs missing phenotype' was a consequence of defects in SGP (Z1/4), the somatic gonad's founder cells. Each SGP gives rise to one DTC and the rest of the cell types of the somatic gonad. It has been reported that alterations in SGP number and position lead to a lack of DTCs (Large \& Mathies, 2014, Mathies et al., 2003). fzr-1(sal19) always produced both SGP, which were ventrally positioned. 
The distinct descendants from SGP are determined by non-canonical Wnt pathway, which is the main pathway involved in cell lineage determination within somatic gonad through asymmetric division. Wnt activation promotes the specification of distal cells. Defective mutants in Wnt signaling lack DTCs but produce supernumerary proximal cells, i.e., ACs (Chang et al., 2005).

In Drosophila APC/C ${ }^{\mathrm{Fr}}$ modulates the $\mathrm{Wg}$ pathway (Wnt pathway) through degradation of Nek2, which represses Wg signaling. Thus, the depletion of Drosophila APC/C ${ }^{\mathrm{Fzr}}$ resulted in an overactivation of the $\mathrm{Wg}$ pathway (Martins et al., 2017). If this kind of relationship is conserved in C. elegans, we would expect just the opposite result, because over-activation of Wnt signaling yields excess of DTCs at the expense of AC (Asahina et al., 2006). A series of indirect evidence suggested that $A P C / C^{F Z R-1}$ was not affecting Wnt signaling in somatic gonad: more than one AC is never detected, male somatic gonad in fzr-1(sal19) does not suffer cell lineage alterations and seam cells, which requires Wnt signaling, are properly form in fzr-1(sal19). However, we cannot rule out that the Wnt pathway was explicitly affected in the distal cell lineages within somatic gonad.

An alternative explanation for the role of FZR-1 in DTC determination is that APC/ $C^{\text {FZR-1 }}$ was necessary during early somatic gonad development for the strict control between cell cycle and differentiation occurring during the development of any organ. It has been reported that $\mathrm{G} 1 / \mathrm{S}$-cell-cycle regulators are essential to coordinate division and differentiation. The depletion of cellcycle regulators CYD-1, CYE-1, and CKI-1 modifies the final number of DTCs. Such modification in DTC number is mostly explained by cell lineage alterations, although cell cycle abnormalities also occur, especially for CKI-1. At the molecular level, the connection between cell cycle and differentiation in the somatic gonad is through non-canonical Wnt pathway: CYD-1 controls noncanonical Wnt pathway, which in turn inhibits CYE-1 (see the introduction for further detail)(Fujita et al., 2007, Kostić et al., 2003, Tilmann \& Kimble, 2005).

Our results indicate that $\mathrm{APC} / \mathrm{C}^{\mathrm{FZR}-1}$ could act more specifically during DTC determination. Fine tunning of a bipartite system to track Z1aa/Z4pp shows that these cells, which generally commit to DTC, differentiate instead into spermatheca and sheath cell-like fates $f z r-1$ (sal19). As both fates derive from SS precursor cells, it indicates that APC/C $C^{\text {FZR-1 }}$ could actively promote DTC fate 
over the default SS precursor fate. This prompted us to think that by blocking SS precursor fate acquisition, DTC's production would be recovered in fzr1(sal19). However, little is known about factors implicated in SS fate acquisition. We tried to silence $x p n-1$, a factor implicated in sheath and spermatheca cell production (Bender et al., 2004a), but DTCs were not reestablished.

We were able to restore DTC production in fzr-1(sal19) after adapting the bipartite Z1aa/Z4pp tracking system to express fzr-1 specifically. This result further confirms that APC/C $C^{\text {FZR-1 }}$ behaves as a factor that determines DTC fate commitment.

We explored in more detail the new role of $A P C / C^{F Z R-1}$ in DTC fate commitment by considering $\mathrm{APC} / \mathrm{C}^{\mathrm{FZR}-1}$ as a DTC inductor. We showed that forced expression of $f z r-1$ using a transgene is not enough to induce that other cells of the somatic gonad commit a DTC fate. Under this framework, we speculated that only a constitutively active version of FZR-1 should render extra DTCs. FZR-1 is inhibited through CDK-mediated phosphorylation. We designed a putative phospho-null version of FZR-1, FZR-1(8A) (The et al., 2015). In vivo experiments conducted in mice reported that a putative phospho-null version of Cdh1 does not affect somatic tissues (Tanno et al., 2020). We have not detected significant defects either in FZR-1(8A) induced worms. According to our predictions, FZR-1 $(8 \mathrm{~A})$ yielded extra DTCs. We support the notion that ectopic DTCs could proceed from Z1ap/Z4pa, DTCs sisters, that usually commit the SS precursor destiny. Several results support this hypothesis. First, extra DTCs do not come from the division of pre-existing DTCs. Second, after induction of $f z r-1(8 A)$ no more than four DTCs were observed per worm. Third, it is demonstrated that the depletion of cye-1 levels renders extra DTCs that come from Z1ap/Z4pa. After the silencing of cye-1 and simultaneous induction of $f z r-1(8 A)$, no augment was observed in the frequency of extra DTCs and number, which never exceeded four. Moreover, the silencing of cye-1 does not rescue DTC production in fzr-1(sal19). It is demonstrated that FZR-1 is phosphorylated and inhibited by G1 CYD/CDK-4/6. Nonetheless, G1/S CYE1/CDK-2 could also be participating in FZR-1 inhibition(The et al., 2015) cyd-1 and cye-1 mutants behave on the contrary way regarding DTC production. cyd1 mutants miss DTCs while cye-1 produces ectopic DTCs. It is logical to think that FZR-1 is inhibited through CDK-dependent phosphorylation lead by CYE- 


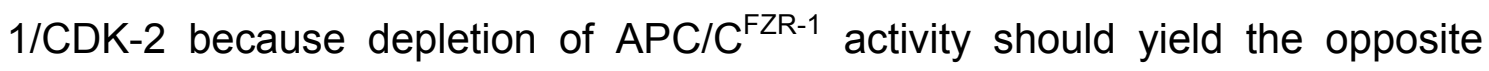
result the corresponding(Fujita et al., 2007).

Altogether, we propose a working model (Fig 4.1) in which the Wnt pathway, through the asymmetric distribution of CYE-1 between Z1aa/Z4pp and Z1ap/Z4pa, controls APC/C $C^{F Z R-1}$ activity. In the case of Z1aa/Z4pp, which receive lower doses of CYE-1, FZR-1 would be hypophosphorylated and active, forcing Z1aa/Z4pp to commit the DTC fate. On the other hand, Z1ap/Z4pa contains higher concentrations of CYE-1, which switch off $A P C / C^{F Z R-1}$, allowing

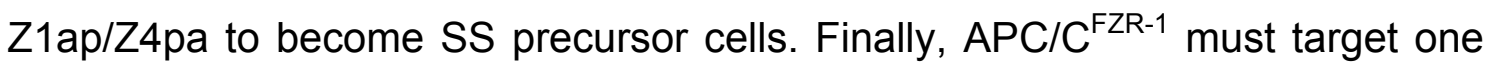
or several repressors in order to activate DTC fate commitment. We highlight from this model the role of $A P C / C^{F Z R-1}$ favoring DTC fate acquisition over SS precursor fate, which is the default destiny when FZR-1 is absent. Strikingly, the fate choice decision DTC-SS in few occasions, leans in favor of DTC commitment in fzr-1(sal19). This stochastic variance could be explained by the fortuitous lower presence of repressor/s in Z1aa/Z4pp that would be degraded by $A P C / C^{F Z R-1}$ or by lower doses FZR-1 that are still present. APC/C ${ }^{F Z R-1}$ has always been reported opposing G1/S transition in many organs but has never been directly implicated in differentiation processes. To give some examples, on one side, the transcription factor $\mathrm{BRO}-1 / \mathrm{CBF} \beta$ is necessary for seam cell fate commitment and division.

Along with larvae development, seam cells divide asymmetrically, giving an anterior daughter cell that differentiates into neuron or hyp cell and a posterior daughter cell that retains the seam fate. bro-1 mutants have fewer seam cells, whose production is rescued in bro-1; lin-35 or bro-1; fzr-1 double mutants (Xia et al., 2007). Another example comes from spermatheca, where nuclear receptor NHR-6/NR4A represses the expression of fzr-1 and lin-35, activating the division of spermatheca cells (Praslicka \& Gissendanner, 2015). Nonetheless, we cannot discard that DTC-SS lineage alterations produced in fzr-1(sal19) are entirely independent of cell-cycle defects. The same could happen for ectopic DTCs produced in $f z r-1(8 A)$.

During cell differentiation, the induction of expression of new proteins is as essential as protein degradation. In this regard, ubiquitin ligases play an essential role during fate specification. It is known for C.elegans that E3 ubiquitin ligases participate in differentiation during embryogenesis. 
Determination of MS (mesoderm) fate after the division of EMS (endomesoderm) is driven by E3 ubiquitin ligases $C R L 1 / S_{C F} F^{L I N-23}$, CRL1/SCF ${ }^{\mathrm{FBX}-3}$, and $\mathrm{CRL} 2 / \mathrm{SCF}^{\mathrm{ZYG}-11}$, which trigger the elimination of transcription factor SKN-1 through targeting OMA-1. Mutants in these E3 ubiquitin ligases keep high levels of SKN-1, which causes a reiteration of EMS fate instead of differentiating into MS (Du et al., 2015). We speculated that

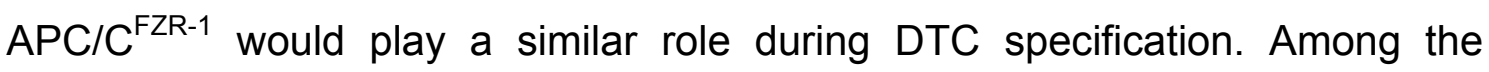
possible targets, we firstly focused on chromatin regulators, because epigenetic modifications are needed for a cell to differentiate and because we

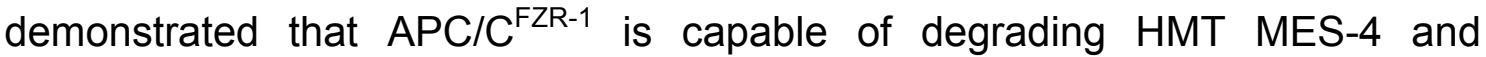
Polycomb subunit MES-3. We think that $A P C / C^{F Z R-1}$ has to degrade some repressor/s to activate DTC fate acquisition. We silenced diverse histone methyltransferases and demethylases containing KEN and/or D-boxes or whose presence has been reported in the somatic gonad. After depletion of met-2 expression, a slight increase in the number of DTCs in fzr-1(sal19) was detected.

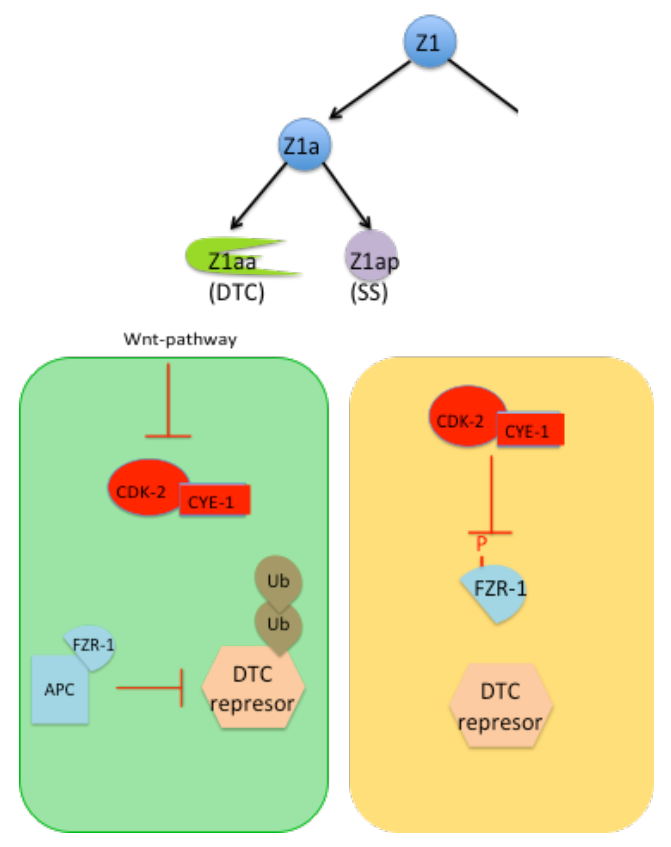

Figure 4.1. Working model depicting the role played by $\mathrm{APC}^{\mathrm{FZR}-1}$ in the production of the DTC.

Interestingly, it has been reported that met-2 mutants display an ectopic activation of lag-2p::GFP transgene (Andersen \& Horvitz, 2007). On the contrary, silencing histone demethylase H3K4 spr-5 and wdr-5.1 involved in 
H3K4 methylation drastically eliminates DTC production in $\mathrm{fzr}-1$ (sal19). In heterozygous $m / n 1 / f z r-1(s a / 19)$, which behave as wild-type, silencing of met-2, spr-5, or wdr-5.1 does not alter DTC's number, indicating that only modify the final number of DTC in combination with $f z r-1(s / 19)$.

\subsection{Role of $A P C / C^{F Z R-1}$ in $\mathrm{mDTC}$ in $C$. elegans production and DTCs in related nematodes}

Curiously, the role of $\mathrm{APC} / \mathrm{C}^{\mathrm{FZR}-1}$ determining DTC fate can only be applied to hermaphrodites, since $f z r-1$ (sal19) males always produce mDTC. The sex-specific requirement of $A P C / C^{F Z R-1}$ could be explained by attending the more complex origin and nature of hDTCs. In hermaphrodites, two consecutive divisions from Z1/Z4 give rise to DTCs, while mDTC originate from a single division of Z1/Z4. Regarding their cell biology, hDTC are more giant cells that extend long processes and serve as a stem-cell niche and leading cell. On the other side, mDTCs only behave as a stem-cell niche. Taken together, specification of $\mathrm{hDTC}$ could be more complicated than $\mathrm{mDTC}$, requiring that APC/C ${ }^{\text {FZR-1 }}$ target one or several factors absent during mDTC genesis. It is also possible that low maternal doses of FZR-1 are enough to produce mDTC.

It would be interesting to explore the role played by $A P C / C^{F Z R-1}$ in the production of DTC in different nematodes. The gonadal structure is highly variable among nematodes related to $C$. elegans. Generally, there are two classes of nematodes regarding gonadal structure: didelphic species, which contain two symmetrical gonadal arms, such as hermaphrodites of C. elegans, and monodelphic species, which only develop one anterior gonadal arm, or in best cases, a reminiscent of a posterior second gonadal arm. To get this morphological variety of gonads, evolution has altered the features and final destiny of cells that constitute somatic gonad. DTC has been subjected to a high degree of evolution. In monodelphic species, anterior DTC (Z1aa) is produced. However, cells that give rise to posterior DTC could suffer a different outcome. In some nematodes, posterior DTC is produced, but its leader function is weaker. In other species, DTC is produced but soon after disappear due to cell death. Finally, other monodelphic nematodes never yield a posterior

DTC

(Félix

$\&$

Sternberg,

1996). 

CONCLUSIONS 



\section{CONCLUSIONS}

1. Polycomb subunit MES-3 is a direct target of $A P C / C^{F Z R-1}$ through its KEN box.

2. Post-transcriptional repression by GLD-1 in combination with protein degradation mediated by APC/C ${ }^{\mathrm{FZR}-1}$ explains the MES-3 pattern in the germline, i.e., lowering MES-3 levels in pachytene.

3. Non-degradation of MES-3, through mutation of its KEN box, leads to fertility defects. Histone methyltransferase MES-4, previously described as a target of $\mathrm{APC} / \mathrm{C}^{\mathrm{FZR}-1}$, is necessary to counteract those fertility defects from the ectopic expression of MES-3. In this regard, APC/C ${ }^{\text {FZR-1 }}$ helps maintain the appropriate balance between MES-4 and MES-3.

4. The null allele $f z r-1$ (sal19) leads to sterility. APC/C $C^{F Z R-1}$ is essential for developing hermaphrodite gonad, as $f z r-1(s a / 19)$ mutants fail to extend gonadal arms. However, fzr-1(sal19) males always produce an extended gonad, indicating that $\mathrm{APC} / \mathrm{C}^{\mathrm{FZR}-1}$ plays a minor role during male gonad development.

5. Defects of hermaphrodite $f z r-1$ (sal19) gonads are mainly attributed to the absence of the Distal Tip Cell (DTC), the stem-cell niche that maintains a pool of germ cells and leads to gonadal outgrowth.

6. The primordial germ cells ( $Z 2$ and $Z 3)$ and the somatic gonad precursors (Z1 and Z4) are produced in fzr-1(sal19), indicating that absence of DTCs stems from alterations during early somatic gonad development.

7. DTC specification mainly depends on the non-canonical Wnt pathway. Indirect evidences suggest that the Wnt pathway is not altered in fzr1(sal19).

8. APC/C ${ }^{\text {FZR-1 }}$ promotes DTC fate acquisition. In $f z r-1$ (sal19), the cells that differentiate into DTC, Z1aa/Z4pp, presumably commit the SS precursor fate. On the other hand, ectopic induction of a putative phospho-null version of $f z r-1$, which should render a constitutively active $f z r-1$, yields extra DTCs. Most probably, extra DTCs come from other cells that typically do not commit the DTC fate. 




\section{APPENDIX}

\subsection{Hypomorphic alleles of $f z r-1$}

The studies about the functionality of FZR-1 in C. elegans have been hampered by the absence of a complete loss-of-function allele. There are two available alleles in the Caenorhabditis Genetic Center. The first of them, fzr1(ku298), consisted of nucleotide substitution $(G \rightarrow A)$, resulting in the replacement of a conserved cysteine in the WD40-repeat domain with a tyrosine (C526Y) (Fig. I). The second allele is fzr-1 (ok380), which contains a premature stop codon that generates a truncated version of the protein without the WD-40-repeat domain (Fig. I). In both cases, homozygous worms for these alleles seem healthy and fertile. The only additional phenotype reported for fzr1(ku298) was a synthetic interaction with lin35 (n745), resulting in sterility (Fay et al., 2002). We have observed that in fzr-1(ku298) worms, GFP fusions of MES-4 invade the pachytene region. However, no pachytene invasion was observed in fzr-1 (ok380) worms carrying the same GFP fusions (Fig 2).

In summary, these results suggested that both alleles were hypomorphic. We discarded the alternative explanation that FZR-1 has a minor role in $C$. elegans because the microinjection of $\mathrm{fzr}-1$ dsRNA into germlines leads to sterile progeny (Fay et al., 2002).

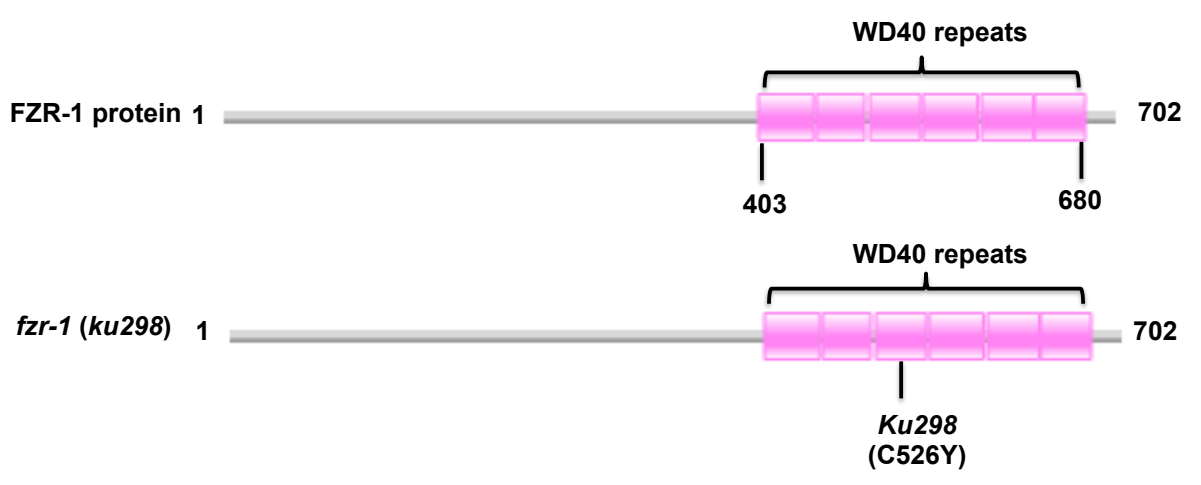

fzr-1 (ok380) 1 268

Truncated protein without WD40 repeats

Figure I. Hypomorphic alleles of fzr-1, fzr-1(ku298) and fzr-1(ok380), available on Caenorhabiditis elegans Center (CGC). 
A
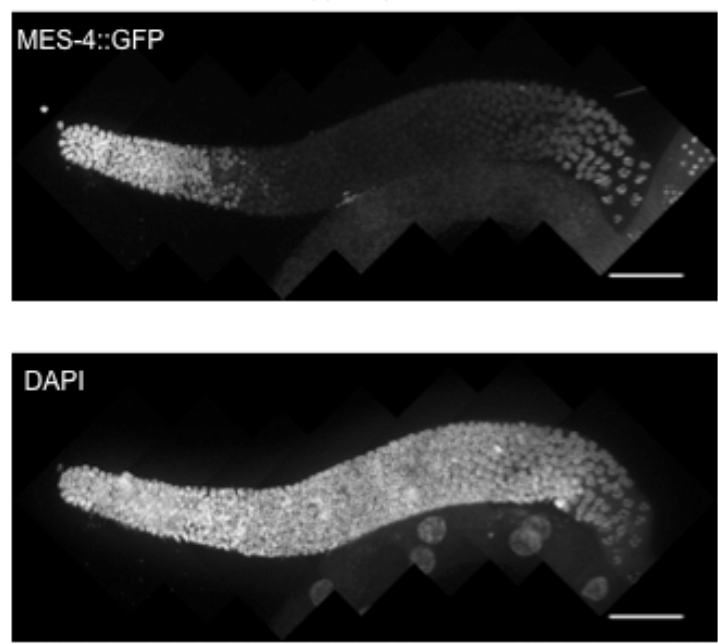

B
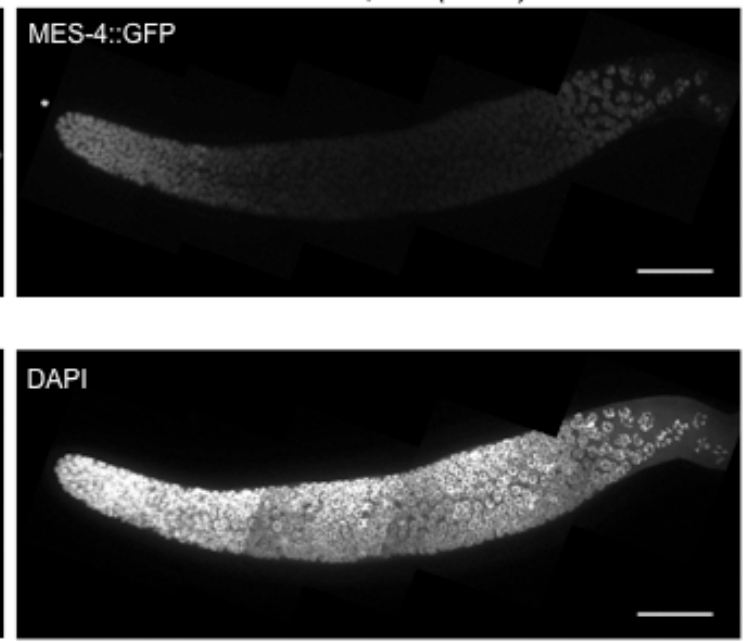

Figure II. fzr-1(ok380) constitutes a hypomorphic allele of fzr-1 that retains enough activity to degrade MES-4. A. (Upper image) In control germlines, MES-4 is present in distal and proximal germline. (Lower image) DAPI-stained gonad. B. (Upper image) In fzr-1(ok380) background, MES-4::GFP displays the same pattern of expression than control germlines. (Lower image) DAPI-stained gonad. Scale bar: 50 $\mu \mathrm{m}$.

\subsection{Bipartite transgenic system to track somatic gonad}

\section{lineages}

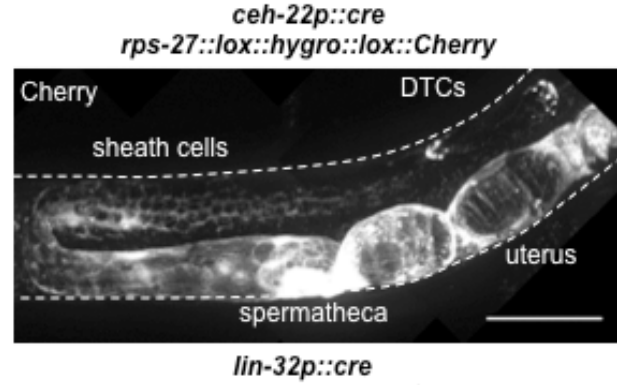

B

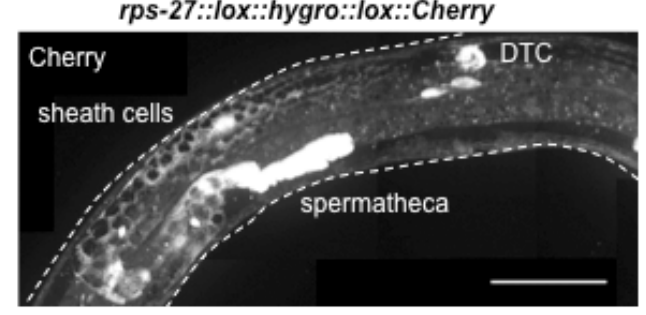

c

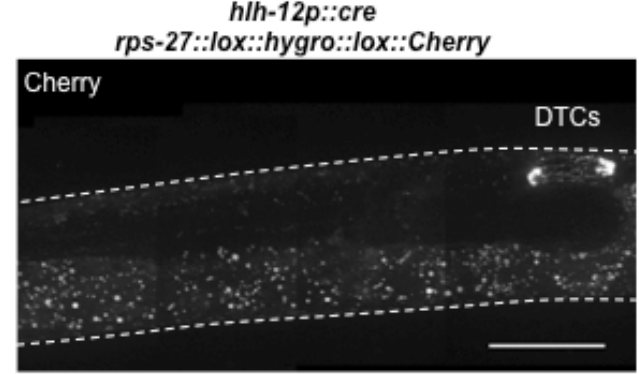

Figure III. Adaptation of the bipartite transgenic system to mark different somatic gonad cells. A. Under promoter of ceh-22, Cherry is expressed in every cell of somatic gonad since the promoter is activated in Z1/4. B. Promoter of lin-32 leads Cherry expression in DTCs, sheath and spermatheca cells, indicating that is expressed in SS precursor cells. C. Promoter of $h / h-12$ is restricted to DTCs. Scale bar: $50 \mu \mathrm{m}$. 


\subsection{Effects of chromatin regulators in the production of DTCs in $f z r-1(s a l 19)$}

A

fzr-1(sal19)/mIn1

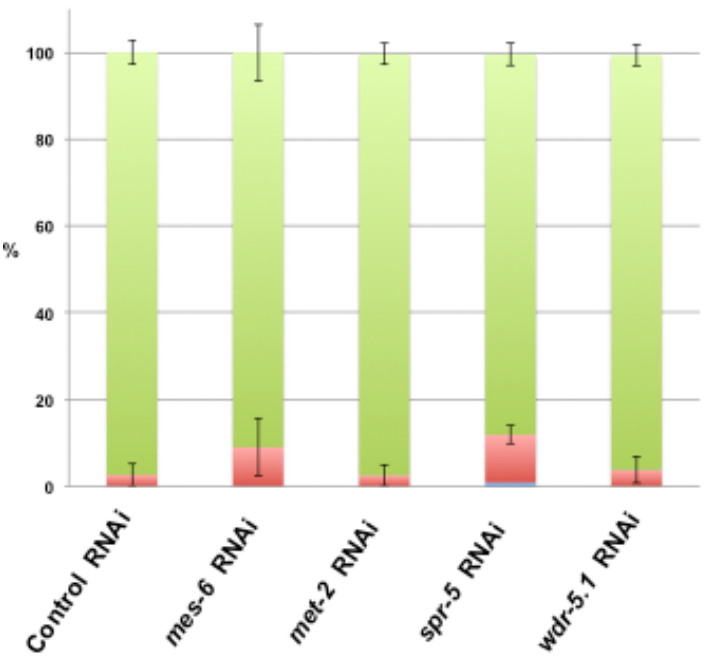

B

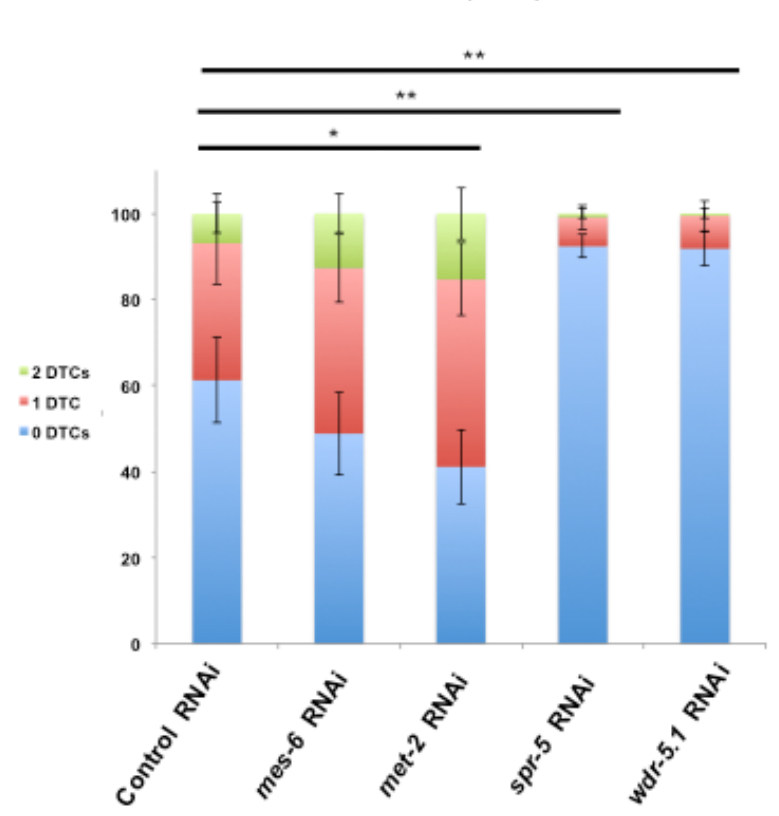

Figure IV. Chromatin regulators affect production of DTC in fzr-1(sal19). Diverse chromatin regulators were silenced by RNAi feeding method. 3-4 fzr-1(sal19)/mIn1 L4s (P0) were grown on RNAi producing bacteria. DTCs per worm were counted for $\mathrm{F} 1$ progeny $f z r-1(\mathrm{sal19}) / \mathrm{m} / \mathrm{ln} 1$ (A) and $\mathrm{fzr}-1$ (sal19) (B). Experiments were conducted at $20^{\circ} \mathrm{C}$. (A) Production of DTCs in F1 progeny $\mathrm{fzr}-1(\mathrm{sal} / 19) / \mathrm{m} / \mathrm{ln} 1$ was not altered in any of these silences. (B) A slight increase in DTC production was observed for F1 fzr-1(sal19) after silencing met-2. On the contrary, spr-5 and $w d r-5.1$ drastically diminishes the number of DTCs in $f z r-$ 1(sal19). 

REFERENCES 



\section{REFERENCES}

Abraham MC, Lu Y, Shaham S, 2007. A morphologically conserved nonapoptotic program promotes linker cell death in Caenorhabditis elegans. Dev Cell 12, 73-86.

Andersen EC, Horvitz HR, 2007. Two C. elegans histone methyltransferases repress lin-3 EGF transcription to inhibit vulval development. Development 134, 2991-9.

Angers S, Moon RT, 2009. Proximal events in Wnt signal transduction. Nat Rev Mol Cell Biol 10, 468-77.

Asahina M, Valenta T, Silhankova M, Korinek V, Jindra M, 2006. Crosstalk between a nuclear receptor and beta-catenin signaling decides cell fates in the C. elegans somatic gonad. Dev Cell 11, 203-11.

Attner MA, Keil W, Benavidez JM, Greenwald I, 2019. HLH-2/E2A Expression Links Stochastic and Deterministic Elements of a Cell Fate Decision during C. elegans Gonadogenesis. Curr Biol 29, 3094-100 e4.

Bando T, Ikeda T, Kagawa H, 2005. The homeoproteins MAB-18 and CEH-14 insulate the dauer collagen gene col-43 from activation by the adjacent promoter of the Spermatheca gene sth-1 in Caenorhabditis elegans. J Mol Biol 348, 101-12.

Barstead RJ, Kleiman L, Waterston RH, 1991. Cloning, sequencing, and mapping of an alpha-actinin gene from the nematode Caenorhabditis elegans. Cell Motil Cytoskeleton 20, 69-78.

Bashir T, Dorrello NV, Amador V, Guardavaccaro D, Pagano M, 2004. Control of the SCF(Skp2-Cks1) ubiquitin ligase by the APC/C(Cdh1) ubiquitin ligase. Nature 428, 190-3. 
Bender AM, Wells O, Fay DS, 2004a. lin-35/Rb and xnp-1/ATR-X function redundantly to control somatic gonad development in C. elegans. Dev Biol 273, $335-49$.

Bender LB, Cao R, Zhang Y, Strome S, 2004b. The MES-2/MES-3/MES-6 complex and regulation of histone $\mathrm{H} 3$ methylation in C. elegans. Curr Biol 14, 1639-43.

Bender LB, Suh J, Carroll CR, et al., 2006. MES-4: an autosome-associated histone methyltransferase that participates in silencing the $X$ chromosomes in the C. elegans germ line. Development 133, 3907-17.

Bessler JB, Andersen EC, Villeneuve AM, 2010. Differential localization and independent acquisition of the H3K9me2 and H3K9me3 chromatin modifications in the Caenorhabditis elegans adult germ line. PLoS Genet 6 , e1000830.

Birnboim HC, Doly J, 1979. A rapid alkaline extraction procedure for screening recombinant plasmid DNA. Nucleic Acids Res 7, 1513-23.

Blanco MA, Sánchez-Díaz A, De Prada JM, Moreno S, 2000. APC(ste9/srw1) promotes degradation of mitotic cyclins in $G(1)$ and is inhibited by cdc2 phosphorylation. EMBO J 19, 3945-55.

Blelloch R, Anna-Arriola SS, Gao D, Li Y, Hodgkin J, Kimble J, 1999. The gon-1 gene is required for gonadal morphogenesis in Caenorhabditis elegans. Dev Biol 216, 382-93.

Boward B, Wu T, Dalton S, 2016. Concise Review: Control of Cell Fate Through Cell Cycle and Pluripotency Networks. Stem Cells 34, 1427-36.

Brenner S, 1974. The genetics of Caenorhabditis elegans. Genetics 77, 71-94. 
Byerly L, Cassada RC, Russell RL, 1976. The life cycle of the nematode Caenorhabditis elegans. I. Wild-type growth and reproduction. Dev Biol 51, 2333.

Byrd DT, Knobel K, Affeldt K, Crittenden SL, Kimble J, 2014. A DTC niche plexus surrounds the germline stem cell pool in Caenorhabditis elegans. PLoS One 9, e88372.

Cappell SD, Chung M, Jaimovich A, Spencer SL, Meyer T, 2016. Irreversible APC(Cdh1) Inactivation Underlies the Point of No Return for Cell-Cycle Entry. Cell 166, 167-80.

Castillo-Lluva S, García-Muse T, Pérez-Martín J, 2004. A member of the Fizzyrelated family of APC activators is regulated by CAMP and is required at different stages of plant infection by Ustilago maydis. J Cell Sci 117, 4143-56.

Cecchetelli AD, Cram EJ, 2017. Regulating distal tip cell migration in space and time. Mech Dev 148, 11-7.

Chang W, Lloyd CE, Zarkower D, 2005. DSH-2 regulates asymmetric cell division in the early C. elegans somatic gonad. Mech Dev 122, 781-9.

Chen J, Mohammad A, Pazdernik N, et al., 2020. GLP-1 Notch-LAG-1 CSL control of the germline stem cell fate is mediated by transcriptional targets Ist-1 and sygl-1. PLoS Genet 16, e1008650.

Chi W, Reinke V, 2006. Promotion of oogenesis and embryogenesis in the C. elegans gonad by EFL-1/DPL-1 (E2F) does not require LIN-35 (pRB). Development 133, 3147-57.

Cohen-Fix O, Peters JM, Kirschner MW, Koshland D, 1996. Anaphase initiation in Saccharomyces cerevisiae is controlled by the APC-dependent degradation of the anaphase inhibitor Pds1p. Genes Dev 10, 3081-93. 
Dalton S, 2015. Linking the Cell Cycle to Cell Fate Decisions. Trends Cell Biol 25, 592-600.

Dickinson DJ, Pani AM, Heppert JK, Higgins CD, Goldstein B, 2015. Streamlined Genome Engineering with a Self-Excising Drug Selection Cassette. Genetics 200, 1035-49.

Du Z, He F, Yu Z, Bowerman B, Bao Z, 2015. E3 ubiquitin ligases promote progression of differentiation during C. elegans embryogenesis. Dev Biol 398, 267-79.

Fay DS, Keenan S, Han M, 2002. fzr-1 and lin-35/Rb function redundantly to control cell proliferation in $\mathrm{C}$. elegans as revealed by a nonbiased synthetic screen. Genes Dev 16, 503-17.

Félix MA, Sternberg PW, 1996. Symmetry breakage in the development of onearmed gonads in nematodes. Development 122, 2129-42.

Fong Y, Bender L, Wang W, Strome S, 2002. Regulation of the different chromatin states of autosomes and $X$ chromosomes in the germ line of $C$. elegans. Science 296, 2235-8.

Frøkær-Jensen C, Davis MW, Ailion M, Jorgensen EM, 2012. Improved Mos1mediated transgenesis in C. elegans. Nat Methods 9, 117-8.

Frøkjaer-Jensen C, Davis MW, Hopkins CE, et al., 2008. Single-copy insertion of transgenes in Caenorhabditis elegans. Nat Genet 40, 1375-83.

Fujita M, Takeshita H, Sawa $H, 2007$. Cyclin $E$ and CDK2 repress the terminal differentiation of quiescent cells after asymmetric division in C. elegans. PLoS One 2, e407.

Furuhashi H, Takasaki T, Rechtsteiner A, et al., 2010. Trans-generational epigenetic regulation of $\mathrm{C}$. elegans primordial germ cells. Epigenetics Chromatin 3, 15. 
Furuta T, Tuck S, Kirchner J, et al., 2000. EMB-30: an APC4 homologue required for metaphase-to-anaphase transitions during meiosis and mitosis in Caenorhabditis elegans. Mol Biol Cell 11, 1401-19.

García-Higuera I, Manchado E, Dubus P, et al., 2008. Genomic stability and tumour suppression by the APC/C cofactor Cdh1. Nat Cell Biol 10, 802-11.

Garvin C, Holdeman R, Strome S, 1998. The phenotype of mes-2, mes-3, mes4 and mes-6, maternal-effect genes required for survival of the germline in Caenorhabditis elegans, is sensitive to chromosome dosage. Genetics 148 , 167-85.

Gaydos LJ, Rechtsteiner A, Egelhofer TA, Carroll CR, Strome S, 2012. Antagonism between MES-4 and Polycomb repressive complex 2 promotes appropriate gene expression in C. elegans germ cells. Cell Rep 2, 1169-77.

Geng Y, Eaton EN, Picón M, et al., 1996. Regulation of cyclin E transcription by E2Fs and retinoblastoma protein. Oncogene 12, 1173-80.

Gleason JE, Eisenmann DM, 2010. Wnt signaling controls the stem cell-like asymmetric division of the epithelial seam cells during $C$. elegans larval development. Dev Biol 348, 58-66.

Golden A, Sadler PL, Wallenfang MR, et al., 2000. Metaphase to anaphase (mat) transition-defective mutants in Caenorhabditis elegans. J Cell Biol 151, 1469-82.

Golden JW, Riddle DL, 1984. The Caenorhabditis elegans dauer larva: developmental effects of pheromone, food, and temperature. Dev Biol 102, 36878.

Guan KL, Jenkins CW, Li Y, et al., 1994. Growth suppression by p18, a p16INK4/MTS1- and p14INK4B/MTS2-related CDK6 inhibitor, correlates with wild-type pRb function. Genes Dev 8, 2939-52. 
Hanahan D, 1983. Studies on transformation of Escherichia coli with plasmids. J Mol Biol 166, 557-80.

Hansen D, Wilson-Berry L, Dang T, Schedl T, 2004. Control of the proliferation versus meiotic development decision in the $\mathrm{C}$. elegans germline through regulation of GLD-1 protein accumulation. Development 131, 93-104.

Harper JW, Adami GR, Wei N, Keyomarsi K, Elledge SJ, 1993. The p21 Cdkinteracting protein Cip1 is a potent inhibitor of G1 cyclin-dependent kinases. Cell 75, 805-16.

Henderson ST, Gao D, Lambie EJ, Kimble J, 1994. lag-2 may encode a signaling ligand for the GLP-1 and LIN-12 receptors of C. elegans. Development 120, 2913-24.

Hirsh D, Oppenheim D, Klass M, 1976. Development of the reproductive system of Caenorhabditis elegans. Dev Biol 49, 200-19.

Hochegger H, Takeda S, Hunt T, 2008. Cyclin-dependent kinases and cellcycle transitions: does one fit all? Nat Rev Mol Cell Biol 9, 910-6.

Hodgkin J, Horvitz HR, Brenner S, 1979. Nondisjunction Mutants of the Nematode CAENORHABDITIS ELEGANS. Genetics 91, 67-94.

Holdeman R, Nehrt S, Strome S, 1998. MES-2, a maternal protein essential for viability of the germline in Caenorhabditis elegans, is homologous to a Drosophila Polycomb group protein. Development 125, 2457-67.

Inoue T, Sherwood DR, Aspöck G, et al., 2002. Gene expression markers for Caenorhabditis elegans vulval cells. Mech Dev 119 Suppl 1, S203-9.

Jan E, Motzny CK, Graves LE, Goodwin EB, 1999. The STAR protein, GLD-1, is a translational regulator of sexual identity in Caenorhabditis elegans. EMBO $J$ 18, 258-69. 
Kamath RS, Ahringer J, 2003. Genome-wide RNAi screening in Caenorhabditis elegans. Methods 30, 313-21.

Kamath RS, Martinez-Campos M, Zipperlen P, Fraser AG, Ahringer J, 2001. Effectiveness of specific RNA-mediated interference through ingested doublestranded RNA in Caenorhabditis elegans. Genome Biol 2, RESEARCH0002.

Kawasaki I, Shim YH, Kirchner J, Kaminker J, Wood WB, Strome S, 1998. PGL1 , a predicted RNA-binding component of germ granules, is essential for fertility in C. elegans. Cell 94, 635-45.

Ketel CS, Andersen EF, Vargas ML, Suh J, Strome S, Simon JA, 2005. Subunit contributions to histone methyltransferase activities of fly and worm polycomb group complexes. Mol Cell Biol 25, 6857-68.

Kim H, Ishidate T, Ghanta KS, et al., 2014. A co-CRISPR strategy for efficient genome editing in Caenorhabditis elegans. Genetics 197, 1069-80.

Kimble J, 1981. Alterations in cell lineage following laser ablation of cells in the somatic gonad of Caenorhabditis elegans. Dev Biol 87, 286-300.

Kimble J, Hirsh D, 1979. The postembryonic cell lineages of the hermaphrodite and male gonads in Caenorhabditis elegans. Dev Biol 70, 396-417.

Kimble J, Simpson P, 1997. The LIN-12/Notch signaling pathway and its regulation. Annu Rev Cell Dev Biol 13, 333-61.

Kimble JE, White JG, 1981. On the control of germ cell development in Caenorhabditis elegans. Dev Biol 81, 208-19.

Kitagawa R, Law E, Tang L, Rose AM, 2002. The Cdc20 homolog, FZY-1, and its interacting protein, IFY-1, are required for proper chromosome segregation in Caenorhabditis elegans. Curr Biol 12, 2118-23. 
Kitamura K, Maekawa H, Shimoda C, 1998. Fission yeast Ste9, a homolog of Hct1/Cdh1 and Fizzy-related, is a novel negative regulator of cell cycle progression during G1-phase. Mol Biol Cell 9, 1065-80.

Koepp DM, Schaefer LK, Ye X, et al., 2001. Phosphorylation-dependent ubiquitination of cyclin E by the SCFFbw7 ubiquitin ligase. Science 294, 173-7.

Korf I, Fan Y, Strome S, 1998. The Polycomb group in Caenorhabditis elegans and maternal control of germline development. Development 125, 2469-78.

Korswagen HC, 2002. Canonical and non-canonical Wnt signaling pathways in Caenorhabditis elegans: variations on a common signaling theme. Bioessays 24, 801-10.

Kostić I, Li S, Roy R, 2003. cki-1 links cell division and cell fate acquisition in the C. elegans somatic gonad. Dev Biol 263, 242-52.

Kraft C, Herzog F, Gieffers C, et al., 2003. Mitotic regulation of the human anaphase-promoting complex by phosphorylation. EMBO J 22, 6598-609.

Lam N, Chesney MA, Kimble J, 2006. Wnt signaling and CEH-22/tinman/Nkx2.5 specify a stem cell niche in C. elegans. Curr Biol 16, 287-95.

Lamont LB, Crittenden SL, Bernstein D, Wickens M, Kimble J, 2004. FBF-1 and FBF-2 regulate the size of the mitotic region in the C. elegans germline. Dev Cell 7, 697-707.

Large EE, Mathies LD, 2010. hunchback and Ikaros-like zinc finger genes control reproductive system development in Caenorhabditis elegans. Dev Biol 339, 51-64.

Large EE, Mathies LD, 2014. Caenorhabditis elegans SWI/SNF subunits control sequential developmental stages in the somatic gonad. G3 (Bethesda) 4, 47183. 
Lasorella A, Stegmüller J, Guardavaccaro D, et al., 2006. Degradation of Id2 by the anaphase-promoting complex couples cell cycle exit and axonal growth. Nature 442, 471-4.

Lee CS, Lu T, Seydoux G, 2017. Nanos promotes epigenetic reprograming of the germline by down-regulation of the THAP transcription factor LIN-15B. Elife 6.

Lee MH, Reynisdóttir I, Massagué J, 1995. Cloning of p57KIP2, a cyclindependent kinase inhibitor with unique domain structure and tissue distribution. Genes Dev 9, 639-49.

Li W, Wu G, Wan $Y, 2007$. The dual effects of Cdh1/APC in myogenesis. FASEB J 21, 3606-17.

Linden LM, Gordon KL, Pani AM, et al., 2017. Identification of regulators of germ stem cell enwrapment by its niche in C. elegans. Dev Biol 429, 271-84.

Liu J, Phillips BT, Amaya MF, Kimble J, Xu W, 2008. The C. elegans SYS-1 protein is a bona fide beta-catenin. Dev Cell 14, 751-61.

Liu Z, Yuan F, Ren J, et al., 2012. GPS-ARM: computational analysis of the APC/C recognition motif by predicting D-boxes and KEN-boxes. PLoS One 7, e34370.

Lukas J, Parry D, Aagaard L, et al., 1995. Retinoblastoma-protein-dependent cell-cycle inhibition by the tumour suppressor p16. Nature 375, 503-6.

Martins T, Meghini F, Florio F, Kimata Y, 2017. The APC/C Coordinates Retinal Differentiation with G1 Arrest through the Nek2-Dependent Modulation of Wingless Signaling. Dev Cell 40, 67-80.

Mathies LD, Henderson ST, Kimble J, 2003. The C. elegans Hand gene controls embryogenesis and early gonadogenesis. Development 130, 2881-92. 
Mccarter J, Bartlett B, Dang T, Schedl T, 1997. Soma-germ cell interactions in Caenorhabditis elegans: multiple events of hermaphrodite germline development require the somatic sheath and spermathecal lineages. Dev Biol 181, 121-43.

Miller JJ, Summers MK, Hansen DV, et al., 2006. Emi1 stably binds and inhibits the anaphase-promoting complex/cyclosome as a pseudosubstrate inhibitor. Genes Dev 20, 2410-20.

Nigon VM, Félix MA, 2017. History of research on C. elegans and other freeliving nematodes as model organisms. WormBook 2017, 1-84.

Nurse P, Thuriaux P, Nasmyth K, 1976. Genetic control of the cell division cycle in the fission yeast Schizosaccharomyces pombe. Mol Gen Genet 146, 167-78.

Paix A, Folkmann A, Rasoloson D, Seydoux G, 2015. High Efficiency, Homology-Directed Genome Editing in Caenorhabditis elegans Using CRISPRCas9 Ribonucleoprotein Complexes. Genetics 201, 47-54.

Patel T, Tursun B, Rahe DP, Hobert O, 2012. Removal of Polycomb repressive complex 2 makes $C$. elegans germ cells susceptible to direct conversion into specific somatic cell types. Cell Rep 2, 1178-86.

Petcherski AG, Kimble J, 2000. LAG-3 is a putative transcriptional activator in the C. elegans Notch pathway. Nature 405, 364-8.

Phillips BT, Kidd AR, 3rd, King R, Hardin J, Kimble J, 2007. Reciprocal asymmetry of SYS-1/beta-catenin and POP-1/TCF controls asymmetric divisions in Caenorhabditis elegans. Proc Natl Acad Sci U S A 104, 3231-6.

Polyak K, Kato JY, Solomon MJ, et al., 1994. p27Kip1, a cyclin-Cdk inhibitor, links transforming growth factor-beta and contact inhibition to cell cycle arrest. Genes Dev 8, 9-22. 
Porta-De-La-Riva M, Fontrodona L, Villanueva A, Cerón J, 2012. Basic Caenorhabditis elegans methods: synchronization and observation. $J$ Vis Exp, e4019.

Praslicka B, Gissendanner CR, 2015. The C. elegans NR4A nuclear receptor gene nhr-6 promotes cell cycle progression in the spermatheca lineage. Dev Dyn 244, 417-30.

Raizen DM, Zimmerman JE, Maycock MH, et al., 2008. Lethargus is a Caenorhabditis elegans sleep-like state. Nature 451, 569-72.

Rechtsteiner A, Costello ME, Egelhofer TA, Garrigues JM, Strome S, Petrella LN, 2019. Repression of Germline Genes in Caenorhabditis elegans Somatic Tissues by H3K9 Dimethylation of Their Promoters. Genetics 212, 125-40.

Rivera-Martín S. MES4 regulation through cell cycle during development in Caenorhabditis elegans. Universidad de Salamanca (2018).

Rizzardi LF, Cook JG, 2012. Flipping the switch from g1 to s phase with e3 ubiquitin ligases. Genes Cancer 3, 634-48.

Rudner AD, Hardwick KG, Murray AW, 2000. Cdc28 activates exit from mitosis in budding yeast. J Cell Biol 149, 1361-76.

Rudner AD, Murray AW, 2000. Phosphorylation by Cdc28 activates the Cdc20dependent activity of the anaphase-promoting complex. J Cell Biol 149, 137790.

Sadasivam S, Decaprio JA, 2013. The DREAM complex: master coordinator of cell cycle-dependent gene expression. Nat Rev Cancer 13, 585-95.

Sallee MD, Littleford HE, Greenwald I, 2017. A bHLH Code for Sexually Dimorphic Form and Function of the C. elegans Somatic Gonad. Curr Biol 27, 1853-60 e5. 
Schade AE, Oser MG, Nicholson HE, Decaprio JA, 2019. Cyclin D-CDK4 relieves cooperative repression of proliferation and cell cycle gene expression by DREAM and RB. Oncogene 38, 4962-76.

Schwab M, Lutum AS, Seufert W, 1997. Yeast Hct1 is a regulator of Clb2 cyclin proteolysis. Cell 90, 683-93.

Shakes DC, Sadler PL, Schumacher JM, Abdolrasulnia M, Golden A, 2003. Developmental defects observed in hypomorphic anaphase-promoting complex mutants are linked to cell cycle abnormalities. Development 130, 1605-20.

Sheaff RJ, Groudine M, Gordon M, Roberts JM, Clurman BE, 1997. Cyclin ECDK2 is a regulator of p27Kip1. Genes Dev 11, 1464-78.

Shibata Y, Sawa H, Nishiwaki K, 2014. HTZ-1/H2A.z and MYS-1/MYST HAT act redundantly to maintain cell fates in somatic gonadal cells through repression of ceh-22 in C. elegans. Development 141, 209-18.

Shin H, Haupt KA, Kershner AM, Kroll-Conner P, Wickens M, Kimble J, 2017. SYGL-1 and LST-1 link niche signaling to PUF RNA repression for stem cell maintenance in Caenorhabditis elegans. PLoS Genet 13, e1007121.

Siegfried KR, Kidd AR, 3rd, Chesney MA, Kimble J, 2004. The sys-1 and sys-3 genes cooperate with Wnt signaling to establish the proximal-distal axis of the Caenorhabditis elegans gonad. Genetics 166, 171-86.

Siegfried KR, Kimble J, 2002. POP-1 controls axis formation during early gonadogenesis in C. elegans. Development 129, 443-53.

Sigl R, Wandke C, Rauch V, Kirk J, Hunt T, Geley S, 2009. Loss of the mammalian APC/C activator FZR1 shortens $G 1$ and lengthens $S$ phase but has little effect on exit from mitosis. J Cell Sci 122, 4208-17. 
Singh AM, Chappell J, Trost R, et al., 2013. Cell-cycle control of developmentally regulated transcription factors accounts for heterogeneity in human pluripotent cells. Stem Cell Reports 1, 532-44.

Singh AM, Sun Y, Li L, et al., 2015. Cell-Cycle Control of Bivalent Epigenetic Domains Regulates the Exit from Pluripotency. Stem Cell Reports 5, 323-36.

Stegmüller J, Huynh MA, Yuan Z, Konishi Y, Bonni A, 2008. TGFbeta-Smad2 signaling regulates the Cdh1-APC/SnoN pathway of axonal morphogenesis. $J$ Neurosci 28, 1961-9.

Strome S, Kelly WG, Ercan S, Lieb JD, 2014. Regulation of the X chromosomes in Caenorhabditis elegans. Cold Spring Harb Perspect Biol 6.

Sulston JE, Horvitz HR, 1977. Post-embryonic cell lineages of the nematode, Caenorhabditis elegans. Dev Biol 56, 110-56.

Sulston JE, Schierenberg E, White JG, Thomson JN, 1983. The embryonic cell lineage of the nematode Caenorhabditis elegans. Dev Biol 100, 64-119.

Swaffer MP, Jones AW, Flynn HR, Snijders AP, Nurse P, 2016. CDK Substrate Phosphorylation and Ordering the Cell Cycle. Cell 167, 1750-61 e16.

Tabuchi TM, Rechtsteiner A, Strome S, Hagstrom KA, 2014. Opposing activities of DRM and MES-4 tune gene expression and X-chromosome repression in Caenorhabditis elegans germ cells. G3 (Bethesda) 4, 143-53.

Tanno N, Kuninaka S, Fujimura S, et al., 2020. Phosphorylation of the Anaphase Promoting Complex activator FZR1/CDH1 is required for Meiosis II entry in mouse male germ cell. Sci Rep 10, 10094.

The I, Ruijtenberg S, Bouchet BP, et al., 2015. Rb and FZR1/Cdh1 determine CDK4/6-cyclin D requirement in C. elegans and human cancer cells. Nat Commun 6, 5906. 
Thornton BR, Ng TM, Matyskiela ME, Carroll CW, Morgan DO, Toczyski DP, 2006. An architectural map of the anaphase-promoting complex. Genes Dev 20, 449-60.

Tilmann C, Kimble J, 2005. Cyclin D regulation of a sexually dimorphic asymmetric cell division. Dev Cell 9, 489-99.

Timmons L, Fire A, 1998. Specific interference by ingested dsRNA. Nature 395, 854.

Updike D, Strome S, 2010. P granule assembly and function in Caenorhabditis elegans germ cells. J Androl 31, 53-60.

Vicencio J, Martínez-Fernández C, Serrat X, Cerón J, 2019. Efficient Generation of Endogenous Fluorescent Reporters by Nested CRISPR in Caenorhabditis elegans. Genetics 211, 1143-54.

Voutev R, Keating R, Hubbard EJ, Vallier LG, 2009. Characterization of the Caenorhabditis elegans Islet LIM-homeodomain ortholog, lim-7. FEBS Lett 583, 456-64.

Walter J, Schermelleh L, Cremer M, Tashiro S, Cremer T, 2003. Chromosome order in HeLa cells changes during mitosis and early $\mathrm{G} 1$, but is stably maintained during subsequent interphase stages. J Cell Biol 160, 685-97.

Wang L, Eckmann CR, Kadyk LC, Wickens M, Kimble J, 2002. A regulatory cytoplasmic poly(A) polymerase in Caenorhabditis elegans. Nature 419, 312-6.

Wäsch R, Cross FR, 2002. APC-dependent proteolysis of the mitotic cyclin Clb2 is essential for mitotic exit. Nature $418,556-62$.

Welchman DP, Mathies LD, Ahringer J, 2007. Similar requirements for CDC-42 and the PAR-3/PAR-6/PKC-3 complex in diverse cell types. Dev Biol 305, 34757. 
White J, Dalton S, 2005. Cell cycle control of embryonic stem cells. Stem Cell Rev 1, 131-8.

Wright JE, Gaidatzis D, Senften M, et al., 2011. A quantitative RNA code for mRNA target selection by the germline fate determinant GLD-1. EMBO J 30 , 533-45.

Wurmthaler LA, Sack M, Gense K, Hartig JS, Gamerdinger M, 2019. A tetracycline-dependent ribozyme switch allows conditional induction of gene expression in Caenorhabditis elegans. Nat Commun 10, 491.

Xia D, Zhang Y, Huang X, Sun Y, Zhang H, 2007. The C. elegans CBFbeta homolog, BRO-1, regulates the proliferation, differentiation and specification of the stem cell-like seam cell lineages. Dev Biol 309, 259-72.

Xu L, Paulsen J, Yoo Y, Goodwin EB, Strome S, 2001. Caenorhabditis elegans MES-3 is a target of GLD-1 and functions epigenetically in germline development. Genetics 159, 1007-17.

Yamaguchi S, Murakami H, Okayama H, 1997. A WD repeat protein controls the cell cycle and differentiation by negatively regulating Cdc2/B-type cyclin complexes. Mol Biol Cell 8, 2475-86.

Yamano H, Tsurumi C, Gannon J, Hunt T, 1998. The role of the destruction box and its neighbouring lysine residues in cyclin B for anaphase ubiquitindependent proteolysis in fission yeast: defining the D-box receptor. EMBO J 17, 5670-8. 
References 